\title{
THE IMPACTS OF A FREE HEALTH INSURANCE POLICY IN
}

\author{
VIETNAM
}

By

NGUYEN HO ANH KHOA

\begin{abstract}
A thesis
submitted to the Victoria University of Wellington in fulfilment of the requirements for the degree of Doctor of Philosophy
\end{abstract}

Victoria University of Wellington

2021 


\begin{abstract}
Social Health Insurance (SHI) is promoted as a policy that tackles the impoverishing effects of catastrophic spending that results from unexpected health shocks. This thesis contributes to the literature on the impact of social health insurance by examining the impact of a policy introduced in Vietnam in 2005.

The new policy provided free health insurance for all children under six years. Using a difference-in-difference estimation strategy and eight national household surveys conducted between 2002 and 2016, I examine a variety of direct, indirect, and spill over effects of the policy. The direct effects of the policy are on insurance coverage, health care use, health care expenses, and self-reported morbidity of targeted children. The indirect effects are the persistent effects of the policy on the same set of health outcomes (and education outcomes) beyond the period of exposure to the policy. A third set of impacts cover spill over effects of the policy - on the health and education outcomes of older children living with targeted children, and a variety of household-level outcomes that reflect various dimensions of the wellbeing of household members.

The results show that a free health insurance policy for young children has significant impacts on the health outcomes of children while they are covered by the policy and these persist, and also extend to educational outcomes beyond the age of eligibility. The policy also has positive spill over effects on older (untargeted) children living in targeted households, and positive effects on household wage income, and income per capita. On the other hand, the policy has no effects on the standard of living, household health expenditure, caloric consumption, and the likelihood of household spending on catastrophic healthcare.
\end{abstract}




\section{Acknowledgements}

Studying for a Ph.D. in New Zealand has been one of the most invaluable experiences of my life. I would like to express my gratitude to many people who have supported me during this journey.

I would like to thank my colleagues from Cantho University, the team of the Vietnam International Education Development (VIED) organization, staff from Victoria University of Wellington as well as Prof Mai Van Nam, Prof Tomomi Tanaka, and Prof Neda Trifkovic for their support, nomination, and financial assistance for my study.

This dissertation would not have been completed without the guidance from my supervisors: Dr Jaikishan Desai, Prof Arthur Grimes, and Dr Harold Cuffe. I am deeply grateful for your academic and mental support during the hardest time of my life. Also, I would like to thank Dr Kate Prickett, Prof Michael Cameron, and Dr Sarah Bales for their wonderful comments and reccommedation on my thesis.

I am thankful to my many great friends. I would like to thank Chung Kien Quoc and Tran Thi Phuong Mai for their lifelong friendship. The memories of us growing up together are amazing that I always hold dear to my heart. I am blessed to know my dearest PhD friend, Rio Yonson, who I have shared many joyful moments, even the long winter walks. For their ongoing support, I would like to thank Andy Walker, Jake Lin, Nguyen Hong Diem, Tran Thi Phuong Thao, Benjamin Arnst, David Alsop, Ganlong Wang, Campbell Whitworth, Doan Vu Nguyen, Nguyen Thi Thu Huyen, Joos van Leeuwen, Nguyen Trang Thu, Le Huy, and Rory Besaans.

I am grateful for the unwavering support from my family and relatives. No matter what happens in life, you always have my back and for that I thank you.

I dedicate this dissertation to my late father, who gives me strength, and to my mother, the most resilient woman that I have ever known. 


\section{Table of Contents}

THE IMPACTS OF A FREE HEALTH INSURANCE POLICY IN VIETNAM ......................................

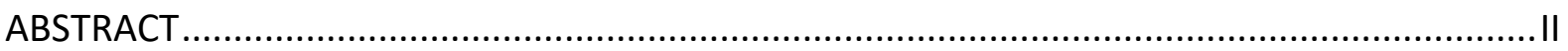

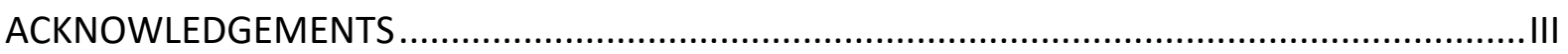

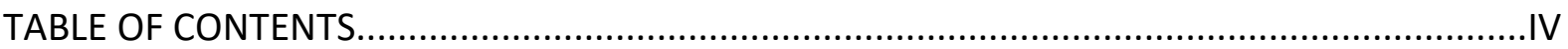

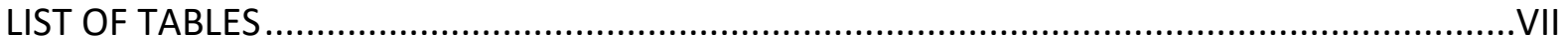

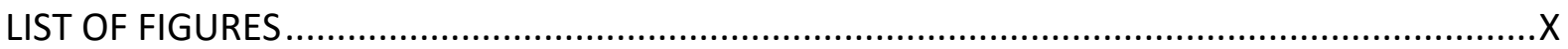

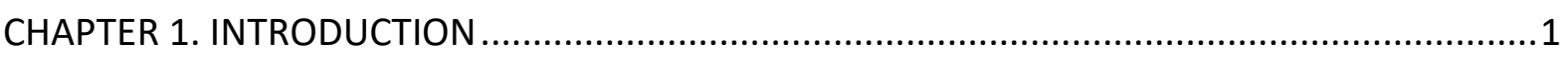

1.1 HEALTH FINANCING IN DEVELOPING NATIONS ..........................................................

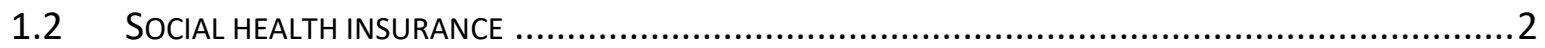

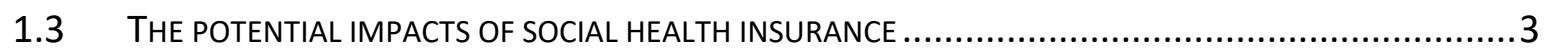

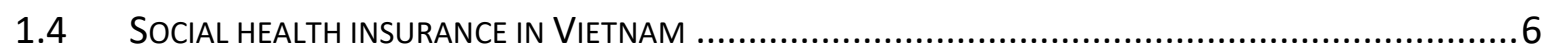

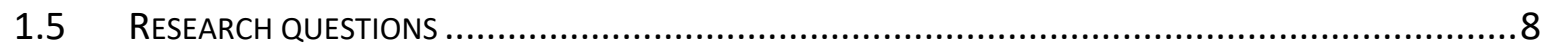

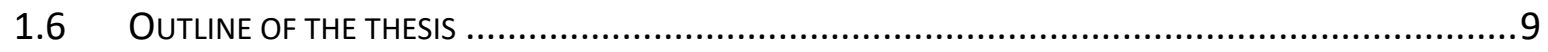

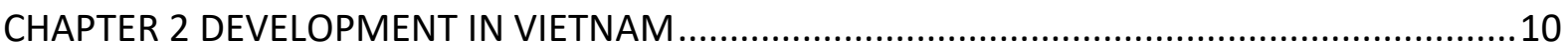

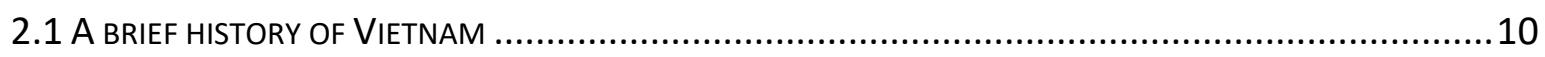

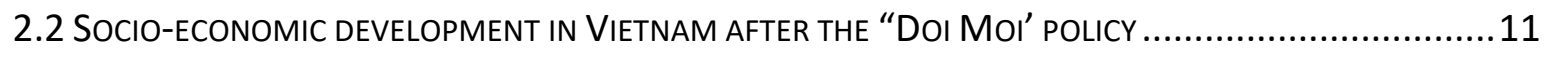

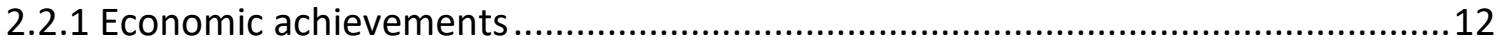

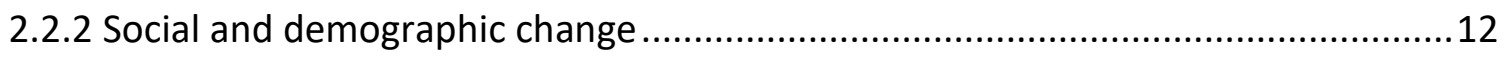

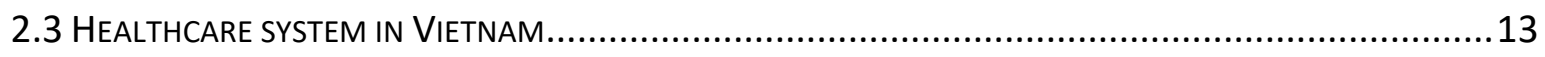

2.3.1 Some changes in the healthcare system in Vietnam ..........................................13

2.3.2 The current structure of Vietnam healthcare system .......................................... 14

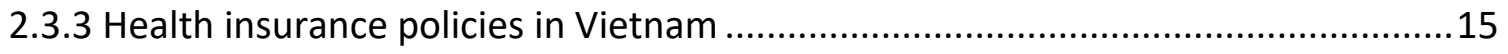

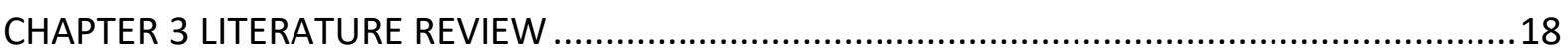




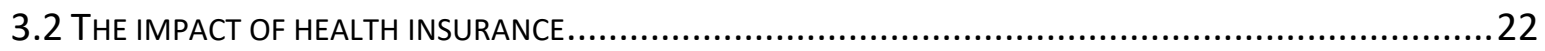

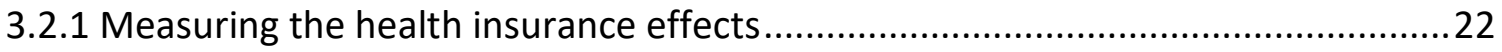

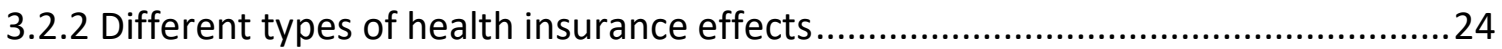

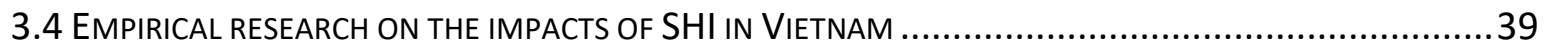

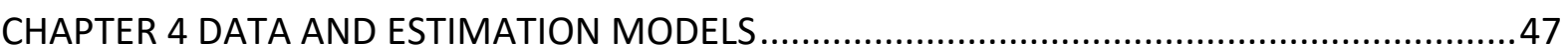

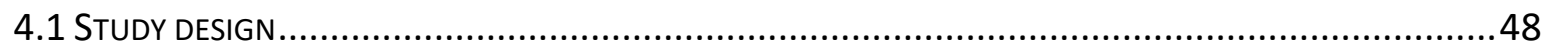

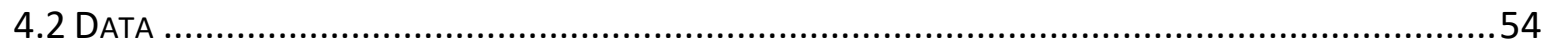

4.2.1 Vietnam Household Living Standard Surveys ....................................................54

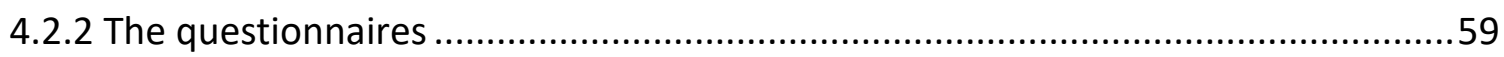

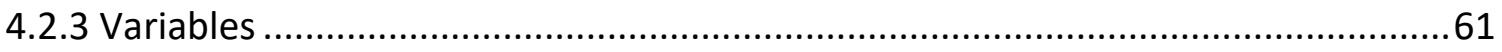

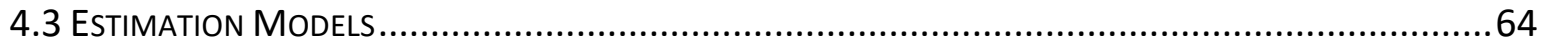

4.3.1 Difference-in-Difference models for the direct effects of the free health insurance policy .64

4.2 Difference-in-Difference models for the indirect (persistent) effects of the free health insurance policy.

4.3.2 Difference-in-Difference models for the spill over effects of the free health insurance policy

CHAPTER 5 THE IMPACTS OF FREE HEALTH INSURANCE POLICY FOR YOUNG CHILDREN ...... 72

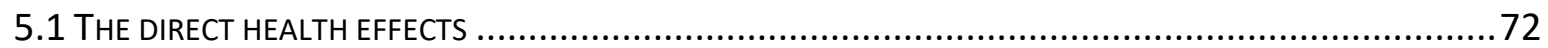

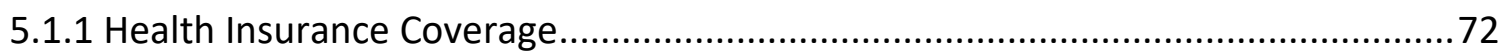

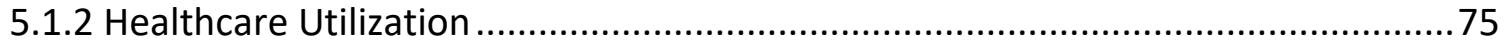

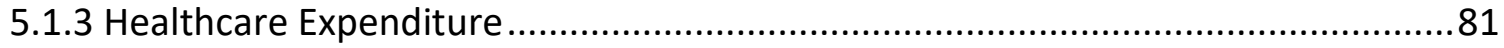

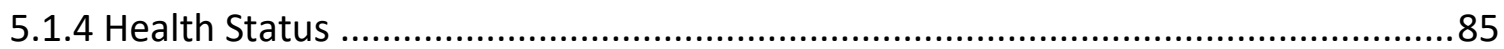

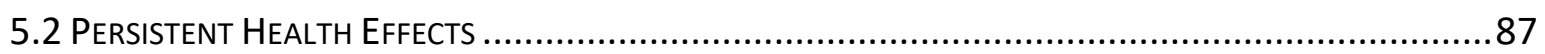

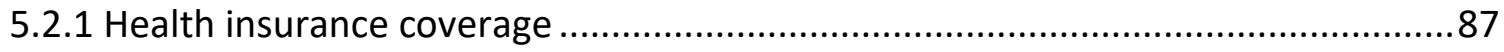


5.2.2 Healthcare utilization .89

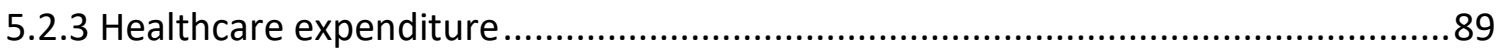

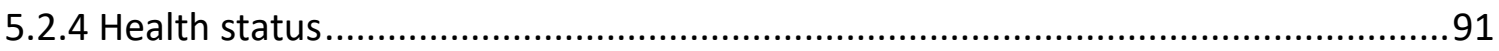

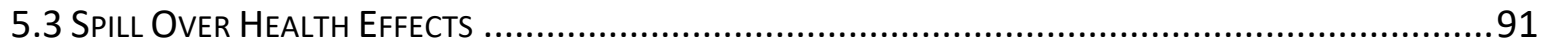

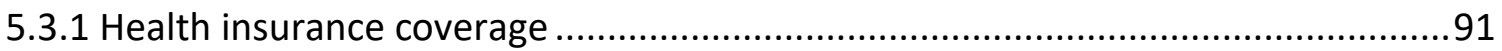

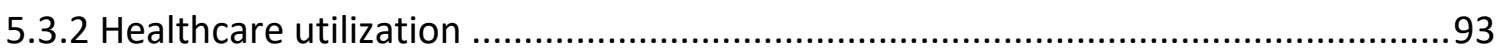

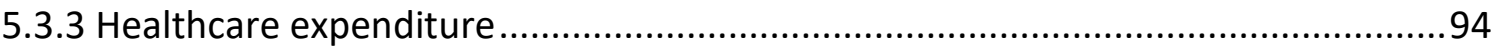

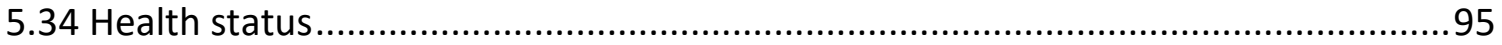

5.4 SPILl OVER EfFECTS ON Other INDIVIDUAL AND HOUSEHOLd OUtCOMES ...................................97

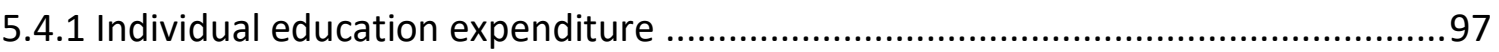

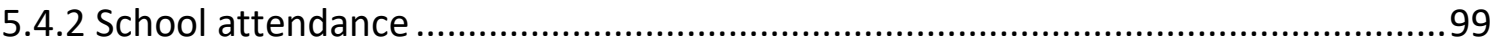

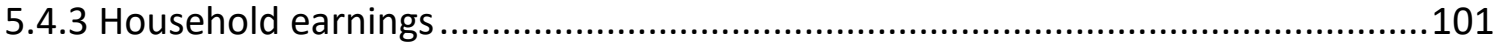

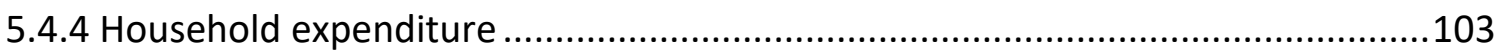

5.4.5 Household health insurance coverage and calorie consumption ........................107

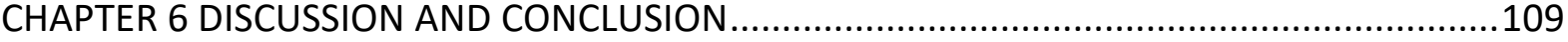

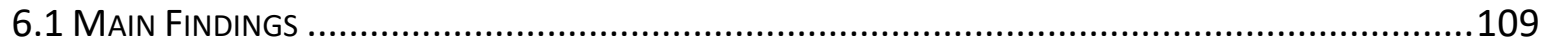

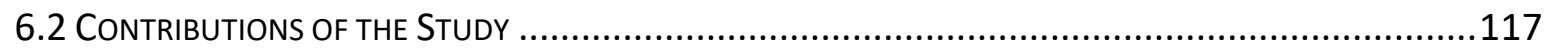

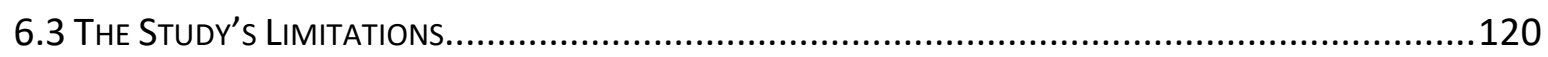

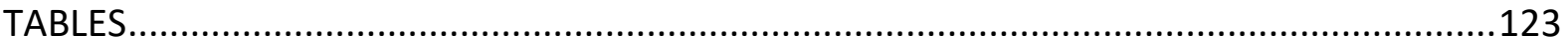

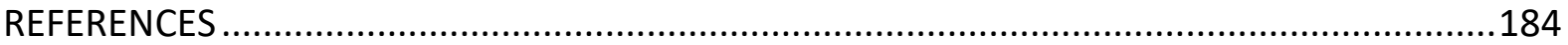

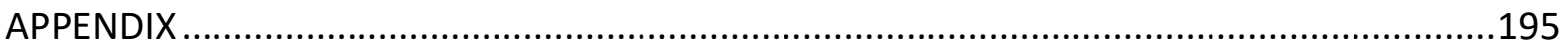




\section{List of Tables}

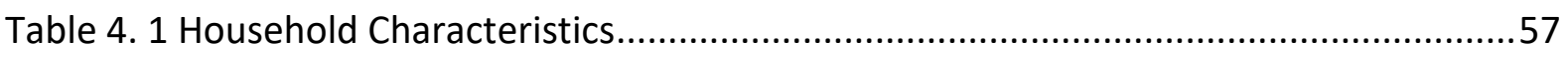

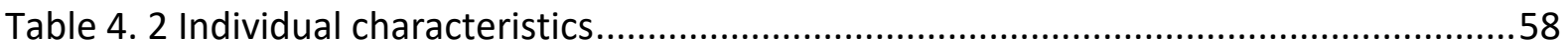

Table 4. 3 The duration of exposing to free health insurance by age and survey year ..........62

Table 5. 1 Direct Health Effect - Health Insurance Status (Children Aged 0-10) ...................124

Table 5. 2 Direct Health Effect - Health Insurance Status (Children Aged 2-10) ..................125

Table 5. 3 Direct Health Effect - Health Insurance Status (Children Aged 4-7) ....................126

Table 5. 4 Direct Health Effect - Health Visit (Children Aged 0-10) ....................................127

Table 5. 5 Direct Health Effect - Health Visit (Children Aged 2-10) ....................................128

Table 5. 6 Direct Health Effect - Health Visit (Children Aged 4-7) ......................................129

Table 5. 7 Direct Health Effect - The number of Outpatient Visit (Children Aged 0-10) .......130

Table 5. 8 Direct Health Effect - The number of Outpatient Visit (Children Aged 2-10) .......131

Table 5. 9 Direct Health Effect - The number of Outpatient Visit (Children Aged 4-7) .........132

Table 5. 10 Direct Health Effect - The number of Inpatient Visit (Children Aged 0-10) ........133

Table 5. 11 Direct Health Effect - The number of Inpatient Visit (Children Aged 2-10) ........134

Table 5. 12 Direct Health Effect - The number of Inpatient Visit (Children Aged 4-7) ..........135

Table 5. 13 Direct Health Effect - Individual Health Expenditure (Children Aged 0-10) .......136

Table 5. 14 Direct Health Effect - Individual Health Expenditure (Children Aged 2-10) .......137

Table 5. 15 Direct Health Effect - Individual Health Expenditure (Children Aged 4-7) .........138

Table 5. 16 Direct Health Effect - Outpatient Expenditure (Children Aged 0-10) .................139

Table 5. 17 Direct Health Effect - Outpatient Expenditure (Children Aged 2-10) .................140

Table 5. 18 Direct Health Effect - Outpatient Expenditure (Children Aged 4-7) ...................141

Table 5. 19 Direct Health Effect - Inpatient Expenditure (Children Aged 0-10) ...................142

Table 5. 20 Direct Health Effect - Inpatient Expenditure (Children Aged 2-10) ...................143

Table 5. 21 Direct Health Effect - Inpatient Expenditure (Children Aged 4-7) .....................144

Table 5. 22 Direct Health Effect - Morbidity Last 30 days (Children Aged 0-10) ...................145

Table 5. 23 Direct Health Effect - Morbidity Last 30 days (Children Aged 2-10) ...................146

Table 5. 24 Direct Health Effect - Morbidity Last 30 days (Children Aged 4-7) ....................147

Table 5. 25 Direct Health Effect - Morbidity Last 12 months (Children Aged 0-10) ..............148 
Table 5. 26 Direct Health Effect - Morbidity Last 12 months (Children Aged 2-10)...... 149

Table 5. 27 Direct Health Effect - Morbidity Last 12 months (Children Aged 4-7) ...............150

Table 5. 28 Persistent Health Effect - Health Insurance Status (Children aged 6-15) ...........151

Table 5. 29 Persistent Health Effect - Health Visit (Children aged 6-15) ..............................152

Table 5. 30 Persistent Health Effect - The number of Outpatient Visit (Children aged 6-15)

Table 5. 31 Persistent Health Effect - The number of Inpatient Visit (Children aged 6-15) ..154

Table 5. 32 Persistent Health Effect - Individual Health Expenditure (Children aged 6-15) .155

Table 5. 33 Persistent Health Effect - Outpatient Expenditure (Children aged 6-15) ...........156

Table 5. 34 Persistent Health Effect - Inpatient Expenditure (Children aged 6-15) ..............157

Table 5. 35 Persistent Health Effect - Morbidity Status last 30 days (Children aged 6-15)...158

Table 5. 36 Persistent Health Effect - Morbidity Status last 12 months (Children aged 6-15)

Table 5. 37 Spill Over Health Effect - Health Insurance Status (Children Aged 6-15) ...........160

Table 5. 38 Spill Over Health Effect - Health Visit (Children Aged 6-15) ..............................161

Table 5. 39 Spill Over Health Effect - The number of Outpatient Visit (Children Aged 6-15)162

Table 5. 40 Spill Over Health Effect - The number of Inpatient Visit (Children Aged 6-15) ..163

Table 5. 41 Spill Over Health Effect - Individual Health Expenditure (Children Aged 6-15)..164

Table 5. 42 Spill Over Health Effect - Outpatient Expenditure (Children Aged 6-15)............165

Table 5. 43 Spill Over Health Effect - Inpatient Expenditure (Children Aged 6-15)...............166

Table 5. 44 Spill Over Health Effect - Morbidity Status last 30 days (Children Aged 6-15) ...167

Table 5. 45 Spill Over Health Effect - Morbidity Status last 12 months (Children Aged 6-15)

Table 5. 46 Persistent Effect on Individual Education Expenditure (Children aged 6-15 with previously exposing to $\mathrm{FHI}$ )

Table 5. 47 Spill Over Effect on Individual Education Expenditure (Children aged 6-15 living with beneficiary) 170

Table 5. 48 Persistent Effect on School Attendance (Children aged 6-15 with previously exposing to $\mathrm{FHI}$ ) .171

Table 5. 49 Spill Over Effect on School Attendance (Children aged 6-15 living with beneficiary) 
Table 5. 51 Spill Over effect on Household Income Per Capita

Table 5. 52 Spill Over effect on Household Expenditure Per Capita ...................................175

Table 5. 53 Spill Over Effect on Household Expenditure Per Adult Equivalent .....................176

Table 5. 54 Spill Over Effect on Household Education Expenditure....................................177

Table 5. 55 Spill Over Effect on Household Healthcare Expenditure ...................................178

Table 5. 56 Spill Over Effect on Catastrophic Health Expenditure ( $>10 \%$ of household

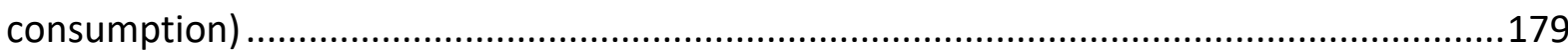

Table 5. 57 Spill Over Effect on Catastrophic Health Expenditure ( $>25 \%$ of household consumption)

Table 5. 58 Spill Over Effect on Catastrophic Health Expenditure ( $>40 \%$ of household consumption)

Table 5. 59 Spill Over Effect on Household Health Insurance Coverage .....

Table 5. 60 Spill Over Effect on Caloric Consumption Per Adult Equivalent 


\section{List of Figures}

Figure 2. 1 Economic Growth in Vietnam from 1984 to 2017 ..........................................195

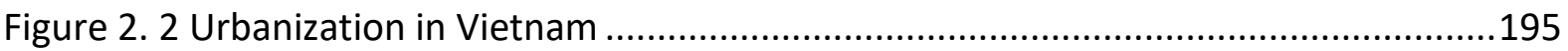

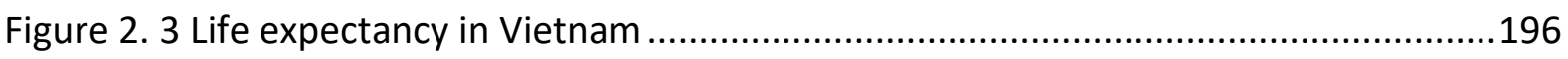

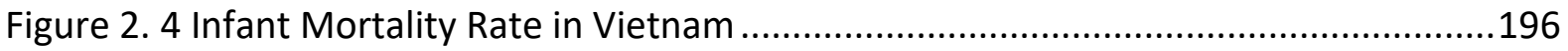

Figure 2. 5 Malnutrition prevalence in Vietnam - Height for Age .....................................197

Figure 2. 6 Malnutrition prevalence in Vietnam - Weight for Age ..................................197

Figure 2. 7 Healthcare expenditure in Vietnam ..............................................................198

Figure 2. 8 Primary types of health care facilities in Vietnam ...........................................199

Figure 2. 9 The number of hospital beds during the last three decades (data from the

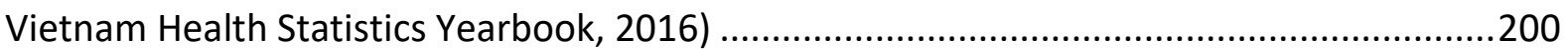

Figure 2. 10 Morbidity trend by types of disease (data from the Vietnam Health Statistics

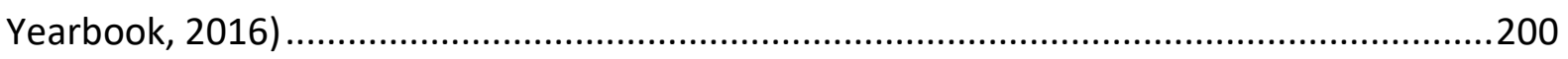

Figure 2. 11 Mortality trend by types of diseases (data from the Vietnam Health Statistics

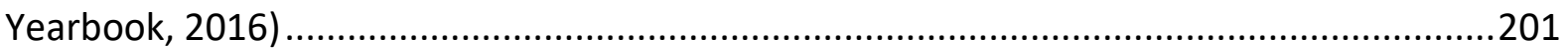

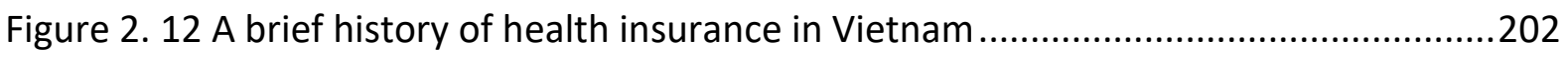

Figure 2. 13 Out-of-pocket payments as a share of national health expenditures (data from

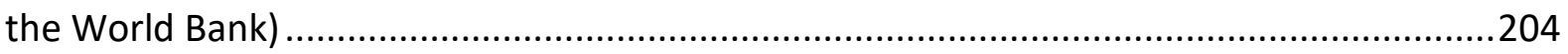

Figure 2. 14 Impoverishment due to illness (data from the World Bank)...........................204

Figure 4. 1 Reported morbidity rates by age (Source: VLSS 93-98 and VHLSS 2004-2008) ....67

Figure 5. 1 Reported Health Insurance Status of Child Groups ......................................... 73

Figure 5. 2 Children's Health Visits in the Last 12 Months ................................................. 76

Figure 5. 3 Children's Outpatient Visits over the Last 12 Months ....................................... 78

Figure 5. 4 The Distribution of Children's Healthcare Utilization ..........................................79

Figure 5. 5 Inpatient Admissions for the Last 12 Months .................................................. 80

Figure 5. 6 Out-of-Pocket Health Expenditure on Different Aged Children ............................82

Figure 5. 7 The Last Month Morbidity Rate of Two Age Groups of Children..........................85

Figure 5. 8 The Last 12 Months Morbidity Rate for Two Groups of Children ........................86

Figure 5. 9 The Persistent Effects on Health Insurance Rates of Children ............................87 
Figure 5. 10 The Reported Health Insurance Status of Two Groups of Children .92

Figure 5. 11 The Health Visits of Two Groups of Children in the Last 12 Months .94

Figure 5. 12 The Out-of-Pocket Health Expenditure for Two Groups of Children .95

Figure 5. 13 The Last Month Morbidity Rate for Two Groups of Children .96

Figure 5. 14 The Last 12 Month Morbidity Rate for Two Groups of Children. .96

Figure 5. 15 Individual Educational Expenditure for Two Family Groups .... .98

Figure 5. 16 Individual Educational Expenditure with Different-Aged Children .98

Figure 5. 17 The School Attendance Rate of Vietnamese Children by Age 100

Figure 5. 18 School Attendance Rate of Vietnamese Children by Year .... 100

Figure 5. 19 Household Wage Income with Different Aged Children 102

Figure 5. 20 Household Income Per Capita with Different Aged Children. .102

Figure 5. 21 Per Capita Household Expenditure Per Capita for Two Different Groups .104

Figure 5. 22 Per Adult Equivalent Household Expenditure for Two Different Groups.... 104

Figure 5. 23 Household Education Expenditure Per Capita for Two Different Households...105 Figure 5. 24 Household Health Expenditure Per Capita for Two Different Households 106

Figure 5. 25 The Probability of Spending over 10\% of Consumption on Healthcare for Two Different Households. .106

Figure 5. 26 Household Health Insurance Rate for Two Different Households 107

Figure 5. 27 The Household Calorie Consumption Per Adult Equivalent for Two Different Households 108 


\section{CHAPTER 1. INTRODUCTION}

There has been an ongoing debate on how to finance healthcare across countries. Some countries favour a healthcare system that relies on private health insurance, some rely on tax financing, whereas some opt for a social health insurance (SHI) model in which participants contribute according to their income. Most countries currently adopt a mixed healthcare financing model that allows for flexibility in mobilizing resources for healthcare provision. In this study, I contribute to this discussion with a case study of SHI in Vietnam.

\subsection{Health financing in developing nations}

Data from the World Bank (2019) reports a wide gap in health investment across countries in the last two decades. Compared with developed countries, in developing countries total healthcare expenditure as a share of GDP and per capita healthcare expenditure was 20 times lower. Public healthcare expenditure accounted for 60 percent of total health expenditure in high-income nations, whereas about 40 percent of healthcare spending in low-income countries was from the public purse. Consequently, the primary source of healthcare funding in developing countries is out-of-pocket payment. In 2014, a household's outlay paid directly to healthcare providers in lower-middle-income countries was 55 percent of total expenditure compared with just 13 percent in high-income countries.

The lack of public funding for healthcare and high out-of-pocket spending can have a detrimental impact on health outcomes in developing nations. Recent data show a strong correlation between healthcare expenditure and life expectancy at birth. Countries that spend less on healthcare tend to have a lower life expectancy at birth and a higher child mortality rate. Although recent economic growth has improved life expectancy in low-income countries, millions of people still suffer the consequences of extreme poverty and premature death (Deaton, 2013). One leading cause of poor health in low-income countries is insufficient funding for sanitation, clean water and the prevention of communicable diseases. In lowincome countries, where public funding for healthcare is limited, households can easily fall into the poverty trap because of catastrophic healthcare spending ${ }^{1}$. In 2010 , there were 808

\footnotetext{
${ }^{1}$ Health expenditure is defined as catastrophic when a household must decrease other consumption over a period of time because of a large healthcare payment. Empirically, there are various thresholds of the proportion
} 
million people (12 percent of the world's population) incurred catastrophic health expenditure compared with 599 million people in 2002 (10 percent of the world's population that year) (A. Wagstaff et al., 2018).

Since governments in developing countries have struggled to mobilize additional resources for healthcare, the World Bank has urged low and middle-income countries to adopt SHI. Recently, the number of nations in Africa, Asia, and Latin America that have adopted SHI is growing (A. Wagstaff, Cotlear, Eozenou, \& Buisman, 2016).

\subsection{Social health insurance}

The demand for healthcare is irregular and unpredictable. The demand for healthcare is uncertain and therefore different from the more stable demand for other essential commodities such as food or shelter. This difference stems from the fact that unlike other essential commodities, the demand for healthcare derives from health which itself is characterized by a degree of uncertainty. Enough income can alleviate the lack of food (hunger) but cannot always prevent illness. Illness that has detrimental effects on health can lead to the loss of earning ability. As a result, healthcare bills that account for a large portion of the budget can impoverish patients and their families, especially those who are economically disadvantaged.

Health insurance can be a solution to prevent catastrophic medical expenses. The idea of health insurance came from the expected utility theory pioneered by Daniel Bernoulli (1738). This theory assumed that individuals aim to maximize the expected value of the utility of income, which includes healthcare costs, as a random reduction component. It further assumed that most individuals are averse to health risks. That assumption means that if an individual must choose between two options: experiencing a probability distribution of medical expenses with a mean of $x$ and paying a health insurance plan with premium $x$ that covers the medical bills, the individual would choose the latter. However, there could be individuals who would ignore the risk of being ill or going through financial hardship through illness, thereby having no demand for health insurance (Arrow, 1963).

of healthcare spending over total expenditure. In this paper, the amount of healthcare spending is considered as catastrophic when it exceeds 10,25 , or 40 percent of total household consumption. 
A lack of demand for health insurance is mainly because of the problem of imperfect and asymmetric information. Individuals know their health status better than insurance companies and this asymmetric information is likely to influence who seeks insurance and who does not, and the premiums companies are likely to charge. For example, those who are healthy, in particular the young, are less likely to seek insurance and pay high premiums. Conversely those whose health is poor, in particular the elderly, are more likely to seek insurance and are also likely to be charged higher premiums because of the higher payouts insurance companies are likely to incur. If those who need insurance, because they are in poor health, are charged higher premiums they are less likely to enrol in insurance schemes. This might be one reason why, in developing countries where poverty and poor health are prevalent, voluntary health insurance programmes are ineffective in expanding coverage and thus burdensome to government budgets (Rothschild \& Stiglitz, 1978).

The World Health Organization (WHO) has encouraged developing nations to implement SHI as a solution to these challenges (WHO, 2005). SHI, first proposed by Bismarck in Germany in 1883, provided coverage and healthcare to those who were required to contribute a percentage of their income irrespective of health risk, socioeconomic status, ability to pay, and living location (Busse, Blümel, Knieps, \& Bärnighausen, 2017). Since then, SHI has been adopted around the world and become more desirable in developing countries across Africa, Asia, and Latin America (Hsiao \& Shaw, 2007). SHI pools as many individuals as possible to spread the financial risk across different groups of the population. This pooling design facilitates the redistribution of resources between the rich and the poor, the young and elderly, as well as the healthy and less healthy (Busse et al., 2017; Hsiao \& Shaw, 2007). Contributions to $\mathrm{SHI}$ funds can generate more resources for healthcare which is, in principle, independent of a government's budget.

\subsection{The potential impacts of social health insurance}

Policy interventions that provide targeted services and resources to households can have direct impacts on the targeted individuals and particular aspects of life they are intended for. But they can also have indirect and spill over impacts that influence the lives of (targeted) individuals beyond the targeted duration, influence other individuals in the household in multiple ways. 
Social health insurance can have a direct impact on the insured, but it might also affect other untargeted individuals and households (Celhay, Martinez, Muñoz, Perez, \& Perez-Cuevas, 2019; Kino, Sato, \& Kawachi, 2018; S. Miller \& Wherry, 2019; Sheu \& Lu, 2014). Unlike existing empirical studies on SHI that focus mainly on the effects on healthcare utilization and health outcomes, this thesis broadens the evaluation of SHI impacts. I examine the impact of $\mathrm{SHI}$ on health outcomes of targeted individuals during the period of targeting and beyond that and look at impacts on health outcomes of others in the household, and spill over impacts on aspects of life beyond health.

At the individual level, SHI can directly impact the health outcomes of beneficiaries. Health indicators such as insurance coverage and healthcare utilization are often used to measure the effects of SHI. Recent empirical evidence shows that SHI can significantly improve the insurance status of individuals in developing countries. There is also evidence that increased access to healthcare encourages people to use more healthcare services. These findings are consistent with the notion that insurance can increase the demand for healthcare by reducing its price (Brook et al., 1984; Finkelstein et al., 2012). Ultimately, what is of interest is the impact of health interventions on health, and some recent studies have shown that SHI does have an impact on indicators such as mortality, morbidity and body mass index (BMI), mental health and nutrition (Erlangga, Suhrcke, Ali, \& Bloor, 2019). It is important to note that there is also some contrary evidence that shows that even though SHI aims to decrease out-ofpocket healthcare expenditure, it provides no financial protection (Guindon, 2014; M. Palmer, Mitra, Mont, \& Groce, 2014).

Besides its direct effects on beneficiaries, SHI might also affect health outcomes indirectly. Since SHI schemes in developing countries often target vulnerable populations of low-income families, young children, and the elderly, its benefits can persist even when these groups no longer receive direct benefits. Previous studies have shown that childhood conditions can have prolonged effects on adult health outcomes (Jackson, 2015; S. Miller \& Wherry, 2019). Thus, I postulate that children exposed to SHI early in life might continue to benefit from the policy even after coverage stops. Specifically, previous exposure can encourage parents to continue to purchase health insurance for these children. If past insurance coverage improved their health status, these children might also have lower healthcare needs and better current health in older years. 
SHI might also impact the health outcomes of other untargeted members in households. In other words, the health benefits of SHI can spill over to those who happen to live in the same family as the beneficiary (Duflo, 2003; Fitzsimons, Malde, Mesnard, \& Vera-Hernández, 2016; Kazianga, de Walque, \& Alderman, 2014; N. Le, Groot, Tomini, \& Tomini, 2019; Monheit \& Vistnes, 2015; Robinson, 2013; Ver Ploeg, 2009). By subsidizing medical expenses, SHI can relax budget constraints and free up household resources. These additional resources can then be allocated for other purposes that might benefit the health outcomes of other members. For example, parents can purchase health insurance for older children if their young children currently benefit from a SHI scheme. Moreover, if young children are immune to infectious diseases because of health insurance, other household members, especially other children, can also stay healthy.

There are also potential spill over effects of SHI on other outcomes such as education, employment and migration status. If SHI can enhance the health condition of the insured, it can positively affect educational outcomes (Case, Fertig, \& Paxson, 2005; Cohodes, Grossman, Kleiner, \& Lovenheim, 2016; Jackson, 2015). Healthy individuals can enrol in school and attend classes regularly; this can translate to better educational achievement. Additionally, empirical evidence shows that Chinese parents tend to invest more in education for healthy children. Thus, I expect that SHI might result in increased individual education expenditure. The health benefits can also extend to the employment outcomes. Healthy individuals might have a better chance to be employed or have more job options than unhealthy individuals. Better health can allow individuals to work more hours or mobilize for a job, thereby increasing their earning ability. The health effects of $\mathrm{SHI}$ can also affect migration among working-age individuals. In developing countries, this can help healthy individuals migrate to large cities to look for a job.

At the household level, SHI can have spill over effects on various outcomes such as living standard, assets, employment, expenditure on healthcare and education, housing conditions and nutritional status (Adams et al., 2018; Fitzsimons et al., 2016; Le et al., 2019; Leininger, Levy, \& Schanzenbach, 2010; Wagstaff \& Pradhan, 2005). Not only can SHI benefit the targeted households via budget constraints, but it might also relieve stress related to illness and its financial burden or even boost the confidence of the decision-makers of families. This might help them make better investment or spending decisions, thereby transforming the 
household's economic condition. I further argue that SHI might have positive effects on the community and the healthcare system. By preventing and providing better treatment for infectious diseases, SHI can maintain healthy households and the whole community. By benefiting health status and employment, $\mathrm{SHI}$ can positively transform a community economically and create a more secure living environment.

\subsection{Social health insurance in Vietnam}

This thesis evaluates the effects of SHI in Vietnam using a free insurance policy for young children as a case study. In 1990s, the country has implemented SHI in different forms. The first compulsory scheme covered only the formal sector in 1992. In 1994, the government introduced a voluntary scheme for school-age children. The compulsory scheme was expanded to include poor households in 2002 and children under age six in 2005. With the Law on Health Insurance in 2008, the country has gradually included other population segments into its SHI scheme. Over more than two decades, health insurance coverage has substantially increased from 12.5 percent of the population in 1998 to 87 percent in 2018 (Ortiz-Ospina \& Roser, 2019).

It should be noted that Vietnam is a unique case study for the following reasons. First, the country has suffered from wars and destruction during its 4,000 years of history. Before Western colonization, Vietnam was ruled by the Chinese for over 1,000 years. In 1884, Vietnam was completely occupied by the French. During the French colonial time, the country was forced to produce and export 57.8 million tons of rice while the population suffered hunger. After World War II ended in 1945, over two million Vietnamese peasants died in a month because of starvation. After that incident, war spread across the nation and the French were defeated in 1954. The north of Vietnam declared independence and the socialist state was established.

Between 1954 and 1975, when the US occupied the south of Vietnam and turned it into a capitalist state, war broke out again between the country's two regions. The Vietnam war ended in 1975 and the country was officially united in 1976. From 1975 to 1986, the economy was severely damaged by the two wars. From May 1975 to 1989, Vietnam continued to fight on two fronts, with the Chinese on the northern border and with the Khmer Rouge on the 
southern border. Life in Vietnam at that time was tough because of shortages of food and other necessities. During this period, Vietnam had a centrally planned economy in which state-owned businesses were the only producers; there was no private property.

Since 1986, under the "Doi Moi" policy, Vietnam has been reformed economically. The country officially adopted a multi-sector market economy while abolishing the central plan and subsidy policies. In April 1988, Resolution 10-NQ/TW recognized agricultural cooperatives as economic entities. They were granted the right to use land and had full control over the crops they produced. In March 1989, the government shifted the focus of the three important economic programmes that promoted agricultural production, consumer goods, and exports. Since 1994, when US President Clinton lifted the trade embargo on Vietnam, the country has been transformed significantly.

Second, Vietnam has achieved remarkable economic growth in the last three decades. Under the 1986 economic and political reform "Doi Moi", real GDP per capita has significantly increased from US\$ 376 in 1984 to US\$2,500 in 2018. From being one of the world's poorest nations, Vietnam has successfully transformed into a lower-middle-income country. Between 1992 and 2014, the poverty rate, defined as living with less than 3.1 purchasing power dollars per day, Vietnam's proportion decreased substantially from 57 to 12 percent. As a result, over 45 million people could escape poverty (WorldBank, 2019).

The country has experienced rapid social and demographic change. The population had increased from 23.5 million in 1945 to 96.5 million in 2019. This increase has caused a sharp rise between 1961 to 2017 in the population density from 103 to 308 people per square kilometre (WorldBank, 2019). In 2018, though about 70 percent of the population was under 35 years old, Vietnam's population is aging quickly. Life expectancy in Vietnam is among the highest in South East Asia; it increased from 52 to 75 years between 1950 and 2019 (Zijdeman \& Ribeira da Silva, 2015). According to the World Bank forecast, the middle-class will comprise 26 percent of the population by 2026 .

The provision of essential services in Vietnam has improved during the last 30 years. From 1990 to 2016, the percentage of households with access to electricity increased from 74 to 100 percent (WorldBank, 2019). Over the same period, the share of the population with 
access to drinking water increased from 63 to 98 percent. This latter improvement resulted in a decline in the mortality rate because of unsafe water from 11 deaths per 100,000 individuals in 1990 to 2 per 100,000 in 2017 (WorldBank, 2019).

Vietnam has had better health indicators along with its better economic growth. Maternal mortality rates declined between 1990 and 2015 from 139 to 54 per 100,000 live births. Similarly, child and infant mortality rates have fallen from 8.6 percent in 1964 to 2.1 percent in 2017. There have been some improvements in health financing in Vietnam. From 1995 to 2006, the government spent less than six percent of GDP on healthcare. During this time, the public share of the healthcare expenditure was around 30 percent and out-of-pocket payments accounted for about 50 percent of total health spending. After 2006, the country tried to control private healthcare spending. This effort resulted in a decline in out-of-pocket expenditure to below 40 percent while public spending increased to over 40 percent of health expenditure. It should be noted that government spending accounted for over 50 percent of the total healthcare expenditure in 2014. The country needs to sustain its high economic growth to maintain this level of public spending on healthcare (WorldBank, 2019).

A third and unique feature of Vietnam is the availability of nationally representative household survey data for a 25-year period. These permit micro-level analysis of development in Vietnam. The first two household surveys were conducted in 1993 and 1998 with technical assistance of the World Bank, and from 2002 onwards by the General Statistics Office of Vietnam. In this thesis I use data from eight surveys from 2002 to 2016 . The surveys contain information on demography, education, healthcare, employment, household assets and expenditure, housing conditions and household participation in poverty reduction programmes. The information in these surveys allows the study of the effects of the free health insurance policy for young Vietnamese children.

\subsection{Research questions}

This study uses the free health insurance policy (for children under six) to study the impacts of $\mathrm{SHI}$ and seeks to answer the following research questions:

a. What are the effects of the policy on the health outcomes of the insured? 
b. What are the indirect effects of the policy on health outcomes of the insured beyond the duration of (initial) coverage?

c. What is the spill over effect of the policy on health outcomes of other children living with insured children, education of children, and household outcomes like income, expenditure and nutritional intake?

I estimate the effects of the policy change using pooled Vietnam Household Living Standards Survey (VHLSS) data from 2002 to 2016 and Difference-in-Differences (DD) models.

In order to measure the direct impacts, I use a sample of children age under 11 observed before and after the policy change. In this sample, children under six belong to the treatment group and children aged six to 10 serve as the control group. In order to estimate the indirect or persistent effects, I use a sample of children aged six to 15 . Children who had free health insurance at a young age form the treatment group and those without free health insurance are the control group. I estimate spill over effects of the policy by using two different samples. In order to estimate the impact of the policy on ineligible children living with eligible siblings, I use a sample of children aged six to 15 . Children who lived with beneficiaries (under-six siblings) belong to the treatment group, and those without a young sibling are the control group. For measuring spill over effect on various household outcomes I use a sample of households observed before and after the 2005 policy change. This sample contains treated households whose young children had free health insurance and control households that had no young children.

\subsection{Outline of the thesis}

This thesis comprises six chapters. After the introduction in Chapter 1, I discuss the development of Vietnam over the last 25 years in Chapter 2. Chapter 3 presents a literature review on the effects of SHI. I then describe the VHLSS dataset and outline the DD models in the Chapter 4. The DD estimates of the effects of free health insurance policy for young children are reported in Chapter 5, and I conclude the thesis with a discussion in Chapter 6. 


\section{Chapter 2 DEVELOPMENT IN VIETNAM}

This chapter presents some background information on Vietnam, including its history and the socio-economic changes, especially after the reforms in the late 1980s. Then I outline some important features of the healthcare system in Vietnam including the main changes in health and health insurance.

\subsection{A brief history of Vietnam}

The history of Vietnam is a story of fighting against many foreign occupations. The first state of Vietnam was formed in the seventh century B.C. From the third century B.C. to 1979 , the country endured hundreds of wars against formidable nations such as China, France, Japan, and the US. Since the second century B.C., various Chinese dynasties ruled Vietnam for over 1000 years (Vu \& Sharrock, 2015).

After the famous "Bach Dang" victory in 938, Vietnam was liberated from the Chinese and became an independent feudal state. Until the $16^{\text {th }}$ century, Vietnamese people lived under seven dynasties: Ngo, Dinh, Le, Ly, Tran, Ho, and Le So. During this period, Vietnam, one of the wealthiest countries in Asia, witnessed developments in agriculture and irrigation systems. People were deeply influenced by three main religions: Buddhism, Taoism and Confucianism. To escape the Chinese influence, Vietnam transformed Chinese scripts into its unique writing system called "Nom" (Vu \& Sharrock, 2015).

Between the $16^{\text {th }}$ and $18^{\text {th }}$ centuries, the feudal regimes in Vietnam declined. Despite economic and cultural developments, such as building cities and seaports that facilitated trade, the country endured civil wars that divided the country. At that time, many European countries transformed quickly into capitalism.

From 1858 to 1945, the French, who used trade and missionaries, ruled Vietnam. For nearly 100 years, the country became a semi-feudal state in which French colonists had complete control over the Nguyen dynasty. Under the French exploitation, millions of Vietnamese people died from starvation. This created a conflict between landlords and farmers that led to the establishment of the Communist Party. This new political party, led by Ho Chi Minh, sparked a revolt and declared independence from the French in September 1945. Later, in 
1954, the victory of "Dien Bien Phu" and the Geneva Accord forced the French to withdraw from Vietnam. The Democratic Republic of Vietnam in the north was formed.

From 1954 till 1975 saw the war between the north and south of Vietnam. In the south, the Republic of South Vietnam was established with military and financial support from the U.S. The war between the two regions lasted for over 20 years and ended in 1975 after the Americans officially withdrew from the south of the country. The Socialist Republic of Vietnam was formed after the country's unification in 1976.

For 10 years after the liberation, people lived in poverty was caused by the devastating impact of wars, the deficiencies of state socialism, and a trade embargo by the U.S. The per capita GDP of Vietnam in 1984 was \$US 376, which was lower than middle and high-income countries by about 5 and 63 times, respectively. Economic hardship was a primary reason that Vietnam implemented a reform policy in the late 1980s. Under the "Doi Moi" policy of 1986, the nation has experienced major positive transformation. From one of the poorest countries in the world, Vietnam has joined the group of lower-middle-income countries with real GDP per capita in 2018 of US\$2,500. Currently, Vietnam is one of the largest exporters of rice, coffee, cashews, and aquacultural products (WorldBank, 2019).

\subsection{Socio-economic development in Vietnam after the "Doi Moi' policy}

During the Vietnam War, the North implemented a subsidized, centrally planned economic structure. This Soviet model relied on state or collective ownership in which all property and production were controlled by the state or cooperatives and the government decided what and how to produce (Turley \& Selden, 2019).

After gaining independence in 1975, Vietnam witnessed many problems with a centrally planned economy. One challenge of this economic model was the lack of production incentives. This created extreme food shortages and drove inflation up to 402 percent. Also, facing the sudden reduction in foreign aid and the collapse of the Soviet Union, Vietnam fell into an economic crisis in the 1980s. In 1986, the country reformed politically and economically under the "Doi Moi" policy. Since then, Vietnam has achieved remarkable socioeconomic development (WorldBank, 2019). 


\subsubsection{Economic achievements}

Over the last three decades, Vietnam has experienced high economic growth compared with other countries in the region. According to the World Bank, the annual growth rate of Vietnam reached its peak at 9.5 percent in 1995 and has fluctuated around six to seven percent for the last decade. Figure 2.1 (Appendix) shows that the country's real GDP per capita substantially increased after the trade embargo was lifted by the U.S. in 1994. Vietnam changed from a poor into a lower middle-income country with GDP per capita increasing by 240 percent from 1994 to 2017. Compared with Cambodia and Laos, two Indochina nations that were also ruled by the French, Vietnam enjoyed a higher average income. Vietnam and Laos have tended to adopt similar policies and have performed better than Cambodia.

Recent data from the World Bank show that exports accounted for over 90 percent of the GDP during 2013 and 2017. Vietnam's main trading partners include the U.S., China and Japan. The primary export products are rice, coffee, aquacultural products, textiles and clothing. The export value of textiles and aquacultural products in 2017 were reported as approximately 26,000 and 8,300 million USD, respectively. Thus, the Vietnam economy is susceptible to changes in trading policies as well as prices and consumer preferences.

\subsubsection{Social and demographic change}

The country has experienced rapid social and demographic change. The population increased from 23.5 million in 1945 to 96.5 million in 2019. This increase has caused a sharp rise in the population density from 103 to 308 people per square kilometre between 1961 and 2017 (WorldBank, 2019). Since more people migrate to urban areas, the increased population might negatively affect the living conditions in Vietnam's big cities. Figure 2.2 (Appendix) shows that the proportion of people living in urban areas significantly increased from 19 percent in 1980 to 35 percent in 2017.

In 2018, about 70 percent of the population was under 35 years old, but Vietnam's population is aging quickly. This can affect the country's healthcare system that currently faces shortages in funding and infrastructure. The government has considered providing free health insurance to the elderly, but this might be infeasible. Thus, the country might be unable to achieve the planned universal health coverage by 2030. Life expectancy in Vietnam is among the highest 
in South East Asia; it increased from 52 to 75 years between 1950 and 2019 (Zijdeman \& Ribeira da Silva, 2015). According a World Bank forecast, the middle-class will reach 26 percent of the population by 2026 .

\subsection{Healthcare system in Vietnam}

\subsubsection{Some changes in the healthcare system in Vietnam}

In 1945, an official healthcare system was established in the North Vietnam. As a French colony in the early 1940s, the whole country had only 47 hospitals with 4,000 beds for a population of 18 million and there was just one doctor per 180,000 people. Life expectancy at birth in 1936 was 34 years. The mortality rate was 26 per 1,000, and the infant mortality rate was 350 per 1,000 live births. When the war with France ended in 1954, Vietnam built a state-financed healthcare system for which the government used tax revenue to subsidize healthcare (Ladinsky \& Levine, 1985).

In 1975, after the Vietnam War, the new government applied the north-style health system to the entire now united country. At this time, the country had economically suffered from four decades of wars. From 1976 to 1986, the economy under-performed, which affected the allocation of resources for the necessary investment in healthcare. The lack of funding for public health resulted in a significant decrease in the number of annual health visits (London, 2008). However, despite the dire fiscal condition and low healthcare investment, the country's life expectancy at birth and infant mortality rates were comparable to wealthier countries. Figure 2.3 shows that, in 1984, Vietnam's life expectancy at birth was only four years lower than the average of high-income countries. In developing countries, the number of infant deaths can primarily drive overall life expectancy (Deaton, 2013). Figure 2.4 (in the Appendix) illustrates that infant mortality rates in Vietnam are comparable to upper-middleincome countries. The rate dropped significantly from 41 to 16 per 1,000 live births between 1984 and 2017.

From 1986, Vietnam reformed its economy. The country soon adopted a mixed healthcare system in which private hospitals could also provide care. In 1989, the government allowed hospitals to collect partial user fees for the first time. This led to hospital autonomy in 2006. 
Many have witnessed a booming economy since the reforms. Various national indicators showed improvements in economic and health outcomes.

Vietnam's annual growth, which has been higher than other ASEAN countries, reached its record highest of 9.5 percent in 1995. Figures 2.5 and 2.6 (Appendix) show a downward trend in the percentage of children under five who suffered from malnutrition. However, health expenditure as a percentage of GDP, has not reached the level of upper-middle and highincome countries. Indeed, Figure 2.7 (Appendix) indicates that health expenditure in Vietnam accounted for about five percent of GDP from 2000 to 2016. Therefore, people might need to spend more on healthcare services from their own pocket. For instance, personal payment accounted for 80 percent of national health expenditure in Vietnam in 1998 (Wagstaff \& Doorslaer, 2003).

\subsubsection{The current structure of Vietnam healthcare system}

Currently, Vietnam has a public-private mixed system of health facilities at four administration levels. Figure 2.8 shows that, at the central level, the Vietnam Ministry of Health $(\mathrm{MOH})$, along with central hospitals, is responsible for providing protection and healthcare for the population. The $\mathrm{MOH}$ also sets the agenda for the long-term development of the country's health sector. At lower levels, health facilities in provinces, districts, and communes are responsible for managing and providing healthcare. These health facilities receive human and financial resources from the $\mathrm{MOH}$ and the relevant people's committees. Commune health stations provide a wide range of primary care, including maternal and child care, family planning, immunization and the treatment of common infections and diseases.

According to WHO, in 2019, the country has 47 central hospitals, 419 provincial hospitals and 864 district hospitals. There are also 182 private hospitals concentrated in urban areas. In recent years, public hospitals have become overcrowded because of the rapid population increase and the changing pattern of diseases (Takashima, Wada, Tra, \& Smith, 2017).

The latest data from WHO report that the number of hospital beds per 10,000 population of Vietnam in 2005 was comparable to lower and middle-income countries. However, the ratio was lower than the global average measure by seven beds. Figure 2.9 (Appendix) shows that the number of hospital beds, especially public ones, has increased slowly over the last three 
decades. On the other hand, Figures 2.10 and 2.11 (Appendix) illustrate that communicable diseases are less prevalent and non-communicable diseases have become the main cause of morbidity and mortality over the last 40 years. Given the high population density, the change in disease patterns might put much pressure on healthcare staff and overload Vietnam's health facilities.

\subsubsection{Health insurance policies in Vietnam}

There are typically three models of health insurance systems in the world. The first model is a national health service or single-payer in which the government pays for healthcare services using tax revenue as in England and Canada. The second model is the social health insurance system, whose primary healthcare provider can either be public or private insurance funds or companies. The third is a market-based model in which private organizations and insurance companies provide healthcare services as in the U.S.

In Vietnam, the current health insurance system comprises SHI and private health insurance. Vietnam first implemented SHI in 1992 because the country was unable to subsidize healthcare. There are currently two SHI schemes: compulsory or voluntary. The compulsory scheme covers the formal sector, the poor and children under six. Despite a mandate, the compulsory element of SHI is not legally enforceable. Meanwhile, the voluntary scheme includes the informal sector, schoolchildren, students and households. Today's health insurance system in Vietnam is the result of many policy changes. Some significant changes related to insurance policies.

After the 1986 economic reforms, Vietnam started SHI in 1992 to mobilize funds for healthcare and reduce out-of-pocket payments. Appendix Figure 12 presents a brief history of health insurance in Vietnam. Currently, there are two types of health insurance in Vietnam: SHI provided by the Government and private health insurance offered by private insurance companies. SHI differs from the private one since it is not-for-profit. The government uses it as a tool to increase access to healthcare services and prevent financial hardship through illness. The history of health insurance in Vietnam can be depicted as a series of events over three periods. 
Before 1986, there was no health insurance in the country. After gaining independence in 1975 , Vietnam became a socialist country with a centrally planned economy. In 1976, the new government abolished the existing private insurance companies from the old regime in the south. It established a non-life insurance company called BAVINA. In 1997, this company was then merged with Bao Viet Insurance Company in the north to become the first non-life insurance company in the country. The year 1986 witnessed many reforms in Vietnam, including the in the healthcare system.

Figure 2.12 shows that, from 1989 to 2000 , the introduction of health insurance did not have any significant impact on coverage. In 1989, the Government allowed hospitals to collect user fees for the first time. Social and private health insurance were introduced in 1992 and 1996, respectively. SHI has been compulsory for the informal sector since 1992. Currently, workers in the formal sector need to contribute 4.5 percent of monthly wages; employers are responsible for covering 3.5 percent and the employees pay the rest. The voluntary scheme began to cover students and schoolchildren in 1994. In the meantime, the first regulations for insurance businesses in 1993 was a turning point for the private insurance market. Later, some new insurance companies were established. The first foreign insurance company opened its branch in 1999.

From 2001 to 2014, the country witnessed a significant improvement in health insurance coverage. The coverage rate substantially increased from 12.5 percent in 1998 to approximately 70 percent in 2013 . The improved coverage resulted from expansion of SHI during the last 20 years. SHI has dominated health insurance; from 2005 to 2014, the private insurance market covered only five percent of the population. The highlight of this period was the free health insurance mandate for the poor and young children.

Since 2002, poor households can receive free health insurance. Approximately 1.5 million poor individuals (about 15 percent of the population) in 2004 could receive free health insurance cards in Vietnam (Wagstaff, 2007a). After the introduction of the law on children protection in 2004, under-six children received free health insurance a year later. The insurance premium for the poor and for young children equals 4.5 percent of the monthly minimum wage. The government subsidizes this premium entirely using tax revenue. In 2008, 
the law on health insurance allowed farmers and unemployed household members to join the voluntary health insurance scheme.

The government expected that the rapid expansion in insurance coverage would improve access to healthcare services and contain costs. Existing studies show that SHI in Vietnam might have increased outpatient visits, but not inpatient admissions. Besides, these studies consistently found no evidence of the effect of insurance on health expenditure.

A recent report from the World Bank indicated that hospital autonomy in Vietnam had increased hospital revenue by between 1.8 and 3.0 times (WorldBank, 2011). This means that health users paid more for healthcare services. Figure 2.13 (Appendix) shows that out-ofpocket payments are indeed the primary source of healthcare financing in Vietnam, accounting for 40 percent of national health expenditure. Despite the implementation of SHI in 1992, the data do not suggest the programme reduced out-of-pocket payments.

Figure 2.14 (Appendix) further shows that 10 - 15 percent of the population spent over 10 percent of household income or consumption on healthcare, and five percent of the population fell into poverty because of healthcare costs over the last two decades. Overcrowded public hospitals and increasing fees ${ }^{2}$ might be the reason that SHI has had no effect on inpatient care.

\footnotetext{
${ }^{2}$ Between 1993 and 1998, while the deregulation of the pharmaceutical industry partly reduced drug prices by $30 \%$, the hospital autonomy policy contributed to increased fees for public hospitals, and private clinics and doctors, by more than 1000 and 600 percent, respectively.
} 


\section{Chapter 3 LITERATURE REVIEW}

This chapter discusses empirical research on the impact of SHI. The first section introduces a theoretical framework for health insurance as well as a brief history and development of SHI across countries. Next, the empirical evidence on health insurance effects is presented. This section emphasizes estimation methods that were used in previous studies to measure the impact of health insurance. Since this study aims to provide a broad evaluation of $\mathrm{SHI}$, this section presents empirical evidence on the different health insurance effects, including direct, indirect, and spill over effects. Finally, I compare and contrast current studies on the impact of SHI in Vietnam in terms of estimation methods, data and findings.

\subsection{Theoretical framework of health insurance}

The debate on health insurance. Arrow (1963) introduced the theory of insurance that focused on health outcomes. Since measuring health involves uncertainty, it was argued that health could not be insured (McGuire, 2000). Various conditions were set to ensure the insurability of health status. One of these conditions relied on the assumption that healthcare utilization improves the likelihood of gaining health. Therefore, a person with an illness will use healthcare and incur a financial burden. Arrow (1963) postulated that health insurance efficiently provides financial protection against sickness.

Some studies argued that health insurance might have negative welfare effects because of moral hazard problems (Feldstein, 1971; Pauly, 1968). Moral hazard refers to the overuse of healthcare because health insurance lowers the marginal cost of medical services. The empirical evidence on the price elasticity of healthcare demand further supported this view (Feldstein, 1973; Manning, Newhouse, Duan, Keeler, \& Leibowitz, 1987). However, studies also showed that the need for healthcare increased even in the absence of the moral hazard motivation (de Meza, 1983; Pauly, 1968). Nyman (1999) attributed the largest component of the increase in healthcare utilization to the better access to healthcare through health insurance.

Thus, health insurance theory has evolved around two notions. First, given that health outcomes relate to many uncertainties, health insurance can provide financial protection against health risks (Arrow, 1963). Second, other than the moral hazard problem, the most 
important factor that contributes to increased healthcare demand is improved access to healthcare through health insurance.

The role of health insurance in developing countries. The demand for healthcare is irregular and unpredictable. Healthcare demand is different from that for other commodities such as food or shelter that are stable by nature. This difference stems from the fact that health is uncertain (Arrow, 1963); we do not know what is going on inside our bodies. Enough income can alleviate hunger but cannot prevent illness. Illness that has a detrimental effect on health can lead to the loss of earning ability. As a result, healthcare bills that account for a large portion of the budget can impoverish patients and their families, especially those who are already economically disadvantaged.

Arrow (1963) discussed the role of health insurance as a solution to prevent catastrophic medical expenses. The idea of health insurance came from the expected utility theory pioneered by Daniel Bernoulli (1738). This theory assumed that individuals aim to maximize the expected value of the utility of income, which includes healthcare costs as a random reduction component. It further assumed that most individuals are averse to health risks. The assumption means that if an individual must choose between two options: experiencing a probability distribution of medical expenses with a mean of $x$ and paying a health insurance plan with premium $x$ (or $x+b$ where $b>0$ ) that covers the medical bills, the individual would choose the latter. Individuals, who would ignore the risk of being ill or going through financial hardship because of, have no demand for health insurance.

Rothschild and Stiglitz (1978) demonstrated that the lack of demand for health insurance is mainly because of the problem of imperfect and asymmetric information. For instance, health insurance companies charge higher premiums for the group of less healthy individuals such as the elderly, young children, and ethnic groups, according to their health risks. This would discourage young and healthy people from joining a health insurance plan. The reason is that these people would be unwilling to subsidize people with a higher risk status. This lack of participation can explain why, in developing countries where poverty and poor health are prevalent, voluntary health insurance programmes are ineffective in expanding coverage and burdensome to government budgets. 
Social health insurance. SHI pools as many individuals as possible to spread the financial risk across different groups of the population. This pooling design facilitates the redistribution of resources between the rich and the poor, the young and the elderly, as well as the healthy and less healthy (Busse et al., 2017; Hsiao \& Shaw, 2007). The contributions to SHI funds can generate more resources for healthcare, which is theoretically independent of governments' budgets. However, in practice, it is common that developing countries' governments often subsidize the poor and young children. In these countries, the mobilization of the resources of $\mathrm{SHI}$ is challenging because of low per capita income and a small formal sector. SHI funds can even become unsustainable in the presence of an epidemic or pandemic that substantially increases the demand for healthcare. For instance, the Vietnamese government has been trying to flatten the curve of the Coronavirus pandemic to prevent too many individuals from using hospital care at the same time. Otherwise, this could corrupt the healthcare system and cause the SHI fund to burst.

The mobilization of resources for healthcare in developing countries can be challenging. The 2010 World Health Report documented that the national health expenditure in developing nations needed to exceed five percent of GDP to implement universal health coverage (Mclntyre, Meheus, \& Røttingen, 2017). Figure 2.7 shows that health expenditure was less than four percent of GDP among ASEAN countries but health spending gaps among lowincome and wealthier countries are approximately one to two percent of GDP. To increase health expenditure through tax revenue can be difficult for lower-income countries because of low economic growth and income per capita. As a result, the private health expenditure of these countries could amount to 50 to $80 \%$ of total health expenditure (Hsiao \& Shaw, 2007). For example, Figure 2.13 illustrates that out-of-pocket payments for healthcare as a share of total health expenditure in Vietnam were still above 40 percent despite robust economic growth and recent health system reform.

There can be inequity in access to healthcare between the poor and the non-poor in developing countries. Since health facilities are more available in urban areas, poor who live in rural and remote areas might be unable to reach the facilities. Furthermore, if health facilities in urban areas are heavily subsidized, households that have better information and greater access to healthcare can reap more benefits than the poor. Hsiao and Shaw (2007) provided an example that poor households in Ghana could get only 12 percent of total health 
expenditure whereas rich households get over 50 percent of these resources. Since the government normally subsidizes SHI premiums for the poor, $\mathrm{SHI}$ is expected to lift financial barriers and provide better access to healthcare for the poor in the population.

In the absence of SHI or private health insurance, severe illness, or injuries can impoverish many households in the developing world. These low-income households would sell farm livestock, houses or land to cover healthcare costs. Consequently, they could be pushed below the poverty line. This, in turn, could exacerbate their health problems and put the poor into the poverty trap and poor health (Knaul et al., 2011). Figure 2.10 shows that about five percent of the Vietnam population was impoverished through healthcare costs. In some developing countries where SHI premiums for poor households are fully subsidized, there is evidence that insured poor households were less likely to be impoverished by healthcare costs (Baeza \& Packard, 2006).

Therefore, the World Health Organization in 2005 encouraged developing nations to implement SHI. SHI, first proposed by Bismarck in Germany in 1883, provides coverage and healthcare to those who are required to contribute a percentage of their income irrespective of health risk, socioeconomic status, ability to pay, or living location (Busse et al., 2017). Since then, $\mathrm{SHI}$ has been adopted around the world and become more desirable in developing countries across Africa, Asia, and Latin America (Hsiao \& Shaw, 2007).

Recently, the implementation of SHI has had some achievements in the developing world. Under SHI, health insurance coverage increased to 80 percent of the population in Colombia in 2007 and over 90 percent in Thailand in 2001 (Giedion \& Uribe, 2009; Hanvoravongchai \& Hsiao, 2007). High coverage rates can increase healthcare utilization in developing countries across Africa, Asia, and Latin America (Giedion \& Uribe, 2009; Obermann, Jowett, \& Kwon, 2018; Palmer, 2014). After almost a decade of SHI, out-of-pocket payments for healthcare as a share of total health expenditure decreased by ten percentage points in Thailand and by over 30 percentage points in Colombia (Hsiao \& Shaw, 2007). These studies also showed that $\mathrm{SHI}$ improved coverage and healthcare utilization for the vulnerable groups, such as young children, mothers and poor households. 


\subsection{The impact of health insurance}

Since this study examines SHI in a developing country, this section mainly focuses on the effects of public or SHI policies in low and middle-income countries. The first part discusses the methodologies used in previous studies. Then the existing findings on the health insurance impacts will be categorized into direct, indirect, and spill over effects.

\subsubsection{Measuring the health insurance effects}

Measuring the impact of health insurance is a challenging task. We can think of the purchase of health insurance as an individual decision. Therefore, a person's characteristics can play decisive roles in whether he or she will pay insurance premiums. For example, an individual with a high level of risk aversion is more willing to pay for health insurance than those with a neutral risk attitude. In the absence of health insurance, the healthy risk-averse individual would use more healthcare services than the healthy risk-neutral one. Therefore, the observed difference in healthcare utilization between these two individuals can be because of health insurance as well as their risk tolerance. If data on risk attitude is available, we can isolate the causal effects of health insurance condition on their tolerance for risk.

However, in practice, we do not usually observe or can measure a person's risk attitude along with other meaningful characteristics. Therefore, a simple comparison of healthcare use by the insured and uninsured can yield a misleading estimate of the causal effects of health insurance. This example of selection bias because of unobservables also applies to other health outcomes such as health status and expenditure. In econometrics terminology, this is the selection bias because of insurance endogeneity that occurs when the assignment of health insurance is not independent of unobserved characteristics (Angrist \& Pischke, 2008).

Current empirical studies spent much effort to overcome the self-selection bias of health insurance. These studies relied on randomized controlled experiments, natural experiments, and cross-sectional data (Erlangga et al., 2019; Levy \& Meltzer, 2008). In randomized controlled experiments, the assignment of health insurance to an individual is unrelated to his or her characteristics. Therefore, that study design can control for unobserved factors that would affect either the insurance status or health outcomes of interest. In other words, one 
can plausibly attribute the differences in the health outcomes between the insured and uninsured as the causal effect of health insurance.

Randomized controlled experiments on health insurance are rare because they are costly and time-consuming (Angrist \& Pischke, 2008). Among these studies is the famous health insurance experiment in the US (RAND project) that provided individuals with free care as well as insurance plans with different co-insurance rates. This large-scale, randomized controlled experiment randomly assigned health insurance to 7,700 individuals from 2,750 households from 1971 to 1982 (Brook et al., 1984). The Oregon health insurance experiment in 2008 is another example of an expensive study on the effects of health insurance. This experiment used a lottery draw to randomly select 35,169 low-income individuals from 29,664 households in Oregon. The selected uninsured individuals then had a chance to apply for Medicaid.

Around the same time in Cambodia, the SKY micro-health insurance programme started to expand. This programme used a lottery to manipulate prices for insurance premiums in rural areas. About 2,499 households received a deep discount for health insurance premiums in this random fashion (Levine, Polimeni, \& Ramage, 2016). These three examples of randomized controlled experiments have one thing in common: random assignment ensured that the insurance status of participants was unrelated to their unobserved characteristics.

Alternatively, health insurance studies have applied quasi-experimental techniques when data from randomized controlled experiments are not available. A quasi-experimental study design relies on a natural experiment in which things change abruptly, such as following a disaster or a policy change. A natural experiment is like a randomized control experiment because it can randomly alter the insurance status of individuals (Angrist \& Pischke, 2008).

An example of a quasi-experimental study is using a national policy that provides a subsidy on insurance premiums for the poor. After this policy change, poor individuals can obtain insurance regardless of their background. One can measure the causal effects of insurance by comparing the outcomes of the insured and uninsured before and after the policy change. However, other changes over time can also affect everyone's outcomes. These quasi- 
experimental designs need a plausible comparison group of individuals to account for the time effects that are unrelated to insurance status.

Suppose a near-poor group has similar characteristics to the poor. Since they receive no insurance, the difference in their outcomes over time might reflect the time effects. One can isolate the causal effects of health insurance by comparing the outcomes of the poor with the outcomes of the near-poor. This approach is Difference-in-Differences (DD). It relies on the assumption that the outcomes of the insured group and the comparison group would follow a similar trend if there were no policy changes.

Another method compares the outcomes of individuals with income just above and below a poverty line. At this cut-off point, those with a slightly higher income are ineligible for health insurance. Let us assume that the individuals around the poverty line are comparable regarding their characteristics. One can estimate the causal effects of insurance by comparing the outcomes of these individuals at the cut-off point. This method is the Regression Discontinuity (RD) design.

Empiricists have argued that the estimation of the RD model has more statistical power than the DD model because the individuals studied are comparable. However, it might be easier to generalize the results of the DD study since it includes more individuals than does the RD model (Campbell \& Stanley, 2015). Another approach to measuring the effects of insurance used cross-sectional data with Propensity Score Matching. These observational studies compared the outcomes of the insured and uninsured on a propensity score. This score accounts for the systematic differences in insurance status among the individuals. The idea behind this method is that individuals with a similar propensity score are comparable in terms of their characteristics (Austin, 2011).

\subsubsection{Different types of health insurance effects}

Health insurance policies can have direct, indirect, or spill over effects on the access to and use of healthcare services, medical spending, and health status. 


\subsubsection{The direct effects}

This section presents the direct impact of health insurance on health as well as other outcomes. The direct effect refers to the intended impact of insurance policies on the beneficiaries.

Health insurance experiments in the U.S. In 1971, the RAND project conducted the health insurance experiment in six areas of the U.S. The project chose the areas so that there was a regional and urban/rural balance. This large-scale experiment randomly assigned health insurance to 7,700 individuals from 2,750 households from 1971 to 1982 . These individuals might receive free healthcare or various cost-sharing plans. These cost-sharing plans required the insured to co-pay 25,50 , or 95 percent of health expenses. Random assignment into these plans ensured that the insurance coverage was unrelated to the characteristics of individuals or households. This practice enabled researchers to attribute any observed differences in health outcomes as the causal impacts of health insurance.

At the end of the experiment, the project gathered information on participants' healthcare utilization and costs. The participants also went through a comprehensive medical examination. The results showed that individuals with free care had one to two more health visits and 20 percent more hospitalizations than those in the cost-sharing plans. This can be because of the price effect of health insurance since free care eliminates the financial barrier to care seekers, which, in turn, increases their demand for healthcare. Those in the free care plan spent 20 and 30-percent more on healthcare than those with 25 and 95 percent coinsurance rates, respectively. The findings further indicated that health insurance could improve the health status of low-income individuals. The poorest and sickest individuals with hypertension in the free care plan witnessed more significant reductions in blood pressure than those in the cost-sharing plans. These poor participants had better vision and received more dental care while experiencing less severe symptoms. Likewise, health insurance might alleviate stress and reduce sick days for individuals with cost-sharing plans. However, there was no evidence on the impact of health insurance on risky behaviours, such as smoking and excessive eating, (Brook et al., 2006; Manning et al., 1987). 
More recently, there was a randomized controlled experiment on health insurance in Oregon. In 2008, the Oregon health insurance experiment randomly offered low-income individuals a chance to apply for Medicaid. Medicaid provided participants a comprehensive benefits package without any coinsurance. Among 89,824 individuals who signed up, the experiment selected 35,169 individuals from 29,664 households using a lottery draw. These individuals were uninsured in the previous six months and had income below the federal poverty line. After one year of implementing the experiment, the results showed that selected individuals reported higher health insurance rates than unselected individuals by 25 percentage points. With lottery selection as an instrumental variable, the results showed that health insurance could increase the probabilities of using outpatient and inpatient care by 35 and 30 percent, respectively. The evidence indicated that insurance might lower the out-of-pocket health expense by 35 percent while decreasing the probability of having medical debt by 25 percent. The experiment found that Medicaid enrolment was associated with a 32 percent increase in reported overall happiness (Finkelstein et al., 2012).

Many believe these two randomized controlled experiments provide plausible estimates on the impacts of health insurance. Health insurance can probably increase the access and demand for healthcare services because it lowers the price of healthcare. Nevertheless, whether health insurance improves health outcomes is still debatable. Health is uncertain since we are unable to know what is happening inside our bodies. Thus, it is difficult to measure health status objectively. Evidence shows that more care could have detrimental effects on health (Fisher, 2003). Therefore, whether the increase in healthcare utilization because of insurance can improve health remains open.

The direct health effects of health insurance in African countries. I present empirical evidence on the impact of public health insurance in Namibia, Burkina Faso and Ghana. I select a mixed health insurance system in Namibia, a community-based health insurance system in Burkina Faso and Ghana's National Health Insurance Scheme as three examples of public health insurance on different scales.

Gustafsson-Wright, Janssens, and van der Gaag (2010) examined the relationship between health shocks and insurance status on out-of-pocket medical spending in Namibia. Health insurance in that country can be purchased from either open or closed health funds. The 
government runs a closed fund that covers only the formal sector, whereas open funds such as Namibia Medical Care or Namibia Health Plan do not have any restrictions on membership. These funds pay medical expenses directly to healthcare providers for the services used by the insured. Since the study relied on a cross-sectional dataset of a random sample of 1769 households and 7343 individuals in the Greater Windhoek area, a linear regression model was separately applied for the insured and uninsured. This stratification strategy aimed to mitigate the adverse selection problem of voluntary health insurance.

The findings show that the insured incurred a higher level of expense for chronic disease and inpatient services than the uninsured. However, the uninsured paid a higher proportion of expenditure per capita for treatments relative to the insured. The evidence suggests that health shocks might disproportionately affect the uninsured since treatment was so expensive that they could not afford it. The study may suffer from the omitted variable bias because of the unobserved health status. This source of bias prevented the researchers from attributing the results as causal health insurance effects.

The lack of randomized experimental data did not allow the study to correct for time-invariant unobserved factors that affect insurance status. Additionally, the results can also be plagued with a simultaneity bias. For instance, high healthcare expenditure might have worsened health status during the study period (Wooldridge, 2012). Those who incur large health spending might sacrifice other spending, e.g., on food, which is a determinant of health. Also, the financial burden can negatively affect their mental health.

Burkina Faso started a community-based health insurance (CBHI) programme in the Nouna District in January 2004. This insurance type operates at the community level on a voluntary and not-for-profit basis. Under the $\mathrm{CBHI}$ scheme, community members pool their resources to provide support and cover for medical expenses (Carrin, Waelkens, \& Criel, 2005; Ekman, 2004). The purposes of this insurance plan were to provide greater access to healthcare, improve health and prevent catastrophic medical expenditure. Participants who lived in $\mathrm{CBHI}$ areas paid premiums of up to \$USD 3 in exchange for a benefits package at certified health facilities. The package covered outpatient and inpatient services without co-payment. 
Empirical evidence showed that the $\mathrm{CBHI}$ in Burkina Faso had no effect on healthcare utilization but did provide the insured with financial protection. In an initial study, Robyn et al. (2011) used propensity score matching ${ }^{3}$ and logistic regression to compare health utilization between insured and uninsured individuals. The authors found that the insurance scheme significantly increased the use of health facilities. However, this observational study is susceptible to bias because of self-selection into the programme. Robyn, Fink, Sié, and Sauerborn (2012) overcame this selection bias problem by using the random rollout of an insurance scheme to explain health outcomes. Since the programme went through three years of implementation, the probability of being selected randomly varied across households over time. The authors use this selection probability and a panel dataset of over 5,600 individuals from 900 households to estimate the effects of insurance. This is the intention-totreat approach. The results from fixed-effects models showed that $\mathrm{CBHI}$ in Nouna had no significant effect on healthcare utilization. This finding was inconsistent with previous evidence from Robyn et al. (2011). Thus, propensity score matching may be unable to account for unobserved factors that co-determine insurance status and health utilization.

Other studies showed that $\mathrm{CBHI}$ in Burkina Faso could provide financial protection for households. Parmar, Reinhold, Souares, Savadogo, and Sauerborn (2012) estimated the effects of the programme on household assets. The authors used a panel dataset of 890 households from 41 villages from 2004 to 2007. The study used three estimation models to address the selection bias of insurance: ordinary least squares (OLS), two-staged least squares with instrumental variable, and the fixed-effects model. These methods are like the one used by Robyn et al. (2012) that considered eligibility through rollout timing as an instrumental variable. The findings show that insurance might increase household assets per capita by one percent (fixed-effects model) to more than 20 percent (OLS model). Fink, Robyn, Sié, and Sauerborn (2013) found that the programme may significantly decrease the likelihood of catastrophic health expenditure by 30 percentage points. However, the results show limited effect on individual health expenses. This study reported no evidence of the impact of CBHI

\footnotetext{
${ }^{3}$ Propensity score matching is a non-parametric approach that matches the insured and uninsured on each observable variable. The technique is successful only if the matches are close on the observables.
} 
on the health outcomes of children and young adults. Interestingly, the authors found that the programme could increase the mortality rate of older individuals in the community.

In Ghana, the implementation of Ghana's National Health Insurance Scheme (NHIS) began in August 2003. This national universal health insurance programme received funding from tax revenue, a compulsory contribution from the formal sector, individual premiums and donors. Under this scheme, there was no co-payment other than the insurance premiums. The benefits package comprised a wide range of outpatient and inpatient services, such as oral and maternity services, as well as emergency care. Currently, exemption from an insurance premium applies to the elderly, children under 18 whose parents participated in the scheme, the poor, and pregnant women (Blanchet, Fink, \& Osei-Akoto, 2012). In an observational study, Blanchet et al. (2012) found that enrolees in Ghana's NHIS were more likely to visit health facilities and have prescriptions than the uninsured. As previously mentioned, this study is susceptible to the self-selection bias of insurance even after controlling by propensity score matching. Fenny, Asante, Enemark, and Hansen (2014) showed that NHIS individuals in three districts of Ghana were more likely to seek treatment from formal health facilities than the uninsured.

Meanwhile, Abrokwah, Moser, and Norton (2014) found that the NHIS in Ghana might provide financial protection. The authors controlled for selection bias by using random variations in implementation timing across districts in the country. Specifically, they relied on the exogenous variation in the programme rollout to explain healthcare utilization and costs. With a cross-sectional dataset from Ghana's Living Standard Survey in 2006, the study found that gaining access to the NHIS significantly increased prenatal care visits and lowered outof-pocket payments. Aryeetey et al. (2016) reported evidence that NHIS might also prevent households from incurring catastrophic medical expenses. Using data from two repeated household surveys, the authors show that 7 to 18 percent of insured households experienced catastrophic expenditure, whereas 29 to 36 percent of uninsured households experienced catastrophic spending. The paper showed that NHIS enrolment might decrease household out-of-pocket payments by 86 percent.

Latin American countries. In Colombia, empirical evidence showed that health insurance had positive effects on health utilization but no impact on health expenditure. G. Miller, Pinto, 
and Vera-Hernandez (2013) found that, among the poor, Colombia's health insurance program Regimen Subsidiado could increase preventive care visits, provide the insured with financial protection from health risks, and improve children's health outcomes. Trujillo, Ortiz, Gómez, and Steinhardt (2010) reported that diabetes patients who enrolled in the country's SHI programme were less likely to use preventive care than patients with no insurance. However, these insured patients had a higher chance of being hospitalized than uninsured individuals. The author argues that this phenomenon could have negative consequences for the insurance fund because inpatient services are more expensive than preventive services.

In Mexico, recent studies have shown that though health insurance provided financial protection to health risks and improved health status, it had limited effect on health utilization. To measure the effects of the Mexican SHI scheme for the poor, Rivera-Hernandez, Rahman, Mor, and Galarraga (2016) used a fixed-effects model with an instrumental variable and data from repeated cross-sectional surveys. They found that insured older adults had higher insulin use than uninsured by 40 percentage points. However, the results also show that the insurance programme had no effect on the treatment of diabetes and hypertension. On the other hand, Galárraga, Sosa-Rubí, Salinas-Rodríguez, and Sesma-Vázquez (2010) evaluated the impact of a health insurance scheme called Seguro Popular (SP) using two national household surveys and two-stage least squares models with an instrumental variable. The authors found that this policy substantially decreased catastrophic expenditure at the national level as well as reducing individual out-pocket-payments for outpatient services and medical expenses. Grogger, Arnold, León, and Ome (2014) reported heterogeneity in the impact of SP on health expenditure between rural and urban households. That study, which used data from a field experiment and national household surveys, indicated that SP significantly decreased catastrophic expenditure in rural areas. The authors also found that the decrease in catastrophic expenditure in rural areas was more pronounced in households who could access health facilities with more staff. The authors further reported that the SP programme also reduced catastrophic spending in urban areas and the SP programme in Mexico was reported to have positive effects on health outcomes. With micro data from the country's population census and weighted maximum likelihood estimator, Pfutze (2014) found that the programme SP might decrease national infant mortality rates by approximately 5 per 1,000 live births. Pfutze (2015) exploited exogenous 
variations in the roll-out of the SP program and data from a national survey on demography to measure the effects of the policy on the probability of miscarriage during pregnancy. The study showed that health insurance entitlement significantly decreased miscarriage rates by .04 percentage points.

Asian countries. In Cambodia, recent evidence using an experiment in which health insurance premiums were randomized, Levine et al. (2016) found that health insurance had positive effects on economic outcomes and health utilization. Specifically, the study showed that households with deeply discounted health insurance were less likely to experience large health expenditure, to take loans or incur debt, and have less land because of health expenses. The results showed that treated households were more likely to visit public health facilities than private facilities compared with households with no discount. However, the authors found no significant evidence of a health insurance effect on health outcomes.

In Indonesia, the findings of Sparrow, Suryahadi, and Widyanti (2013) showed that SHI increased either health utilization or expenditure. Using a Difference-in-Difference (DD) model with panel data from over 8,000 households, the authors found that the Askeskin social health insurance scheme significantly increased outpatient care among the poor and private health spending of urban households. In the Philippines, Quimbo, Peabody, Shimkhada, Florentino, and Solon (2011) found positive health effects of a randomized health insurance intervention for poor children. Using a DD regression with data of hospital users, the authors showed that poor children with better health insurance were less likely to be wasting or have infections. In Laos, Alkenbrack and Lindelow (2015) reported positive effects of communitybased health insurance targeting the country's informal workforce. The authors used data from a survey of 3,000 households and weighted the regression with propensity score matching. Their results showed that health insurance significantly increased health utilization while decreasing health expenses and catastrophic expenditure. In Thailand, the first middleincome country to implement universal health coverage, recent studies showed that health insurance had positive effects on health outcomes. Using a DD model, Ghislandi, Manachotphong, and Perego (2015) found that health insurance significantly increased the probability of an annual health check-up as well as outpatient and inpatient care. The study also found that health insurance did not promote risky health-related behaviours or reduce preventive care. 


\subsubsection{Indirect effects}

The indirect or persistent impact of health insurance refers to the effect on those who had previous exposure to health insurance.

The existing SES-health gradient reported a positive correlation between good health and higher socioeconomic status among adults (Adler et al., 1994). Previous studies indicated that childhood health might contribute to this gradient (Case et al., 2005; Case, Lubotsky, \& Paxson, 2002). Adults who are taller or had better birthweight tended to have a better performance on a set of social, economic, and cognitive measures (Case \& Paxson, 2008; Currie, 2009; Haddad \& Bouis, 1991; Strauss \& Thomas, 2007; Thomas \& Strauss, 1997). In contrast, poverty and illness in childhood might have a persistent impact on adult health status, thereby lowering education achievement and life opportunities (Kuh \& Wadsworth, 1993). Empirical evidence confirmed that health conditions, such as birth weight, might be the determinant of education level and employment (Currie \& Hyson, 1999; Currie \& Madrian, 1999).

Case et al. (2005) reported a lasting impact of childhood health and circumstances on adult health, employment, and socioeconomic status. Using a panel dataset of a birth cohort that was recorded from birth until middle age, the authors found that a poor uterine environment and health status at a young age negatively affected education achievement, adult health and socioeconomic status. Thus, if health insurance can improve health status at a young age, it is likely that its benefits will extend into adulthood.

Recent research indicated that Medicaid entitlement at a young age might have a positive impact on various health outcomes of teenagers and educational attainment in adulthood (Cohodes et al., 2016). That study used an instrumental variable (IV) approach and a dataset of teenagers who were born in the U.S. between 1980 and 1990. Since Medicaid rules varied across states, the author used Medicaid eligibility as the IV for actual eligibility. This method relies on the assumption that the Medicaid rules were not associated with the health outcomes of interest.

Cohodes et al. (2016) showed that Medicaid entitlement at a young age may have a positive impact on various health measures among teenagers. Specifically, Medicaid status can 
decrease body mass index (BMI) and the likelihood of engaging in risky behaviours such as unprotected sex, drinking and smoking. However, it should be noted that some of these estimates were not statistically significantly different from zero. The study also found that Medicaid exposure at a young age may increase educational achievement in adulthood. For instance, a 10-percentage-point increase in Medicaid eligibility reduced the high school dropout rate and increased the college attendance rate.

With a similar approach, S. Miller and Wherry (2019) found that the expansion of health insurance for children through Medicaid had a lasting impact on targeted adult outcomes. Specifically, the results showed that adults whose mothers were eligible for prenatal insurance coverage under Medicaid experienced a lower obesity rate and fewer hospital admissions for the treatment of a range of diseases. Furthermore, the insurance coverage for mothers tended to increase high school completion rates among affected adults.

Recent studies found evidence on the long-term effects of expansions of the Medicaid and CHIP programmes. Since only children who were born after 1983 were eligible for the Medicaid expansion, this cut-off point created a discontinuity in the duration of eligibility of children born before and after this year. Wherry and Meyer (2016) exploited this policy change to measure the long-term effects of Medicaid expansion on future mortality and hospital admissions of those children. The study indicated that Medicaid expansion had longterm effects on health because it decreased mortality and healthcare use among African American young adults.

Johnson and Schoeni (2011) reported that childhood health (low birth weight) and poverty (limited parental investment, lack of health insurance) had an adverse impact on chronic disease prevalence in middle-age. This study extracted information from PSID to create a nationally representative longitudinal dataset of siblings observed in four decades in the U.S. The authors then applied sibling fixed effects models to the dataset to estimate the effects of childhood condition on health outcomes in middle-age. The fixed effect models control for unobserved time-invariant household characteristics such as genetic endowment or family structure. The findings showed that low birth weight significantly increased the likelihood of chronic diseases such as asthma, hypertension, stroke and diabetes. The estimates were robust to the sibling fixed effects and the inclusion of various control variables. Johnson and 
Schoeni (2011) found that limited parental investment, such as the lack of child health insurance coverage, significantly increased the prevalence of chronic diseases in middle-age.

Nelson (2010) and Lin and Liu (2014) examined whether in utero exposure to the 1918 influenza pandemic affected socioeconomic outcomes in adulthood. Using the epidemic as a natural experiment, the authors found that those who were in utero during the influenza pandemic fared worse than other birth cohorts around 1918. Lin and Liu (2014) found that individuals having in utero exposure to influenza in Taiwan were shorter and more vulnerable to severe illnesses. A one-percentage-point increase in influenza incidence was associated with a-0.343-year decrease in schooling. Similarly, Nelson (2010) showed that the 1919 Brazilian birth cohort that was exposed to the pandemic in utero experienced 0.2 fewer years of education and a 20 percent reduction in wages.

Meanwhile, Levine and Schanzenbach (2009) examined the long-term impact of the expansion of public health insurance through both Medicaid and SCHIP on children's educational outcomes. This paper used a dataset of children from the National Assessment of Educational Progress (NAEP) and a triple difference model. This estimation strategy exploited the exogenous variation in insurance eligibility across states and over time to explain children's reading and mathematics test scores. The study found that health insurance eligibility might positively affect reading but not mathematics test scores. For instance, a 50percentage-point increase in eligibility was associated with a 0.09 -standard-deviation increase in reading test scores. This study also provided evidence on improvements in birth weight and mortality rate because of health insurance. The improvements in health outcomes at birth might positively affect educational measures. However, one should be cautious about interpreting the estimate of the effect of health status on educational outcomes since it can be confounded by unobserved characteristics.

Celhay et al. (2019) evaluated the impact of the Medical Insurance Century XXI (SMSXXI) scheme in Mexico on health outcomes and medical expenditure. Under the national public health insurance policy, Seguro Popular, the Mexican government launched the SMSXXI scheme in 2006 to provide coverage for children under age five. This scheme targeted the uninsured and low-income population intending to increase access to medical care, reduce out-of-pocket spending and eliminate health inequity. 
The study used datasets of hospitals, households and children from nationally representative surveys and administrative data sources from 2001 to 2016. The outcome variables were neonatal and infant mortality rates, self-reported morbidity relating to health status, influenza, and diarrhoea, as well as children's height. Other groups of outcomes included household expenditure for healthcare, hospital discharges, and service quality. Since the programme restricted the enrolment to children who were born after December 2006, the study uses this exogenous variation in the change of eligibility rule to explain the outcomes of interest. A difference-in-difference and triple difference models were used.

Celhay et al. (2019) found evidence of the long-term health effects of the insurance scheme on various health outcomes. After eight years of implementation, the programme was reported to increase the height of affected children and low-income individuals by 0.4 and $0.87 \mathrm{~cm}$, respectively. Within three to six years after the SMSXXI began, it tended to decrease the prevalence of influenza and diarrhoea while improving the health status of the affected children. The scheme also reduced household medical expenditure by approximately 14 percent.

\subsubsection{Spill over effects}

Empirical studies on the impact of a health insurance policy or health intervention should pay attention to the spill over effects of the programmes. Miguel and Kremer (2004) gave an example of the spill over effects of a health intervention in Kenya. The project involved the evaluation of the impact of deworming medication on school-age children in the country. Intestinal worms can be transmitted from one person to another through direct contact with contaminated faeces. When children were dewormed, the benefit can extend to the others living in the same household. Therefore, in this example, when medication was delivered to children in one school, the benefits can spill over to another school. The study found that deworming medication significantly reduced the number of worms of children in nonparticipating schools.

Recent studies have paid more attention to the potential spill over effects of health interventions in African countries. Empirical evidence shows that providing health inputs to a targeted member can affect others within the household. For instance, providing iodine 
supplementation to children in Tanzania can increase parental investment in their siblings (Adhvaryu \& Nyshadham, 2016). Similarly, supplying nutrient supplements to mothers of infants in Ghana may improve the height of untargeted children (Adams et al., 2018).

Similarly, a school meal programme in Burkina Faso that allowed children to bring home food, might improve the weight of pre-school age children. Interestingly, this programme, which served children only lunch at school, seemed to have no impact on their young siblings (Kazianga et al., 2014). Since these studies relied on randomized controlled experiments, which differed in design, treatment, and time frame, there were various explanations about how these spill over effects might occur.

Based on these studies, I outline three potential channels through which spill over effects of health interventions can happen. First, providing free health inputs to a child can relax household budget constraints (income effect). For example, although the iodine supplementation programme in Tanzania aimed to improve child endowment, the programme might free household resources that would have been needed for unhealthy children (Adhvaryu \& Nyshadham, 2016).

Alternatively, or additionally, this can increase parental labour supply in time and effort that would have been used for sick children (Adams et al., 2018; Fitzsimons et al., 2016). Consequently, parents might invest additional resources in other children. The findings showed that children whose younger siblings were exposed to iodine supplementation in utero received better vaccination rates while being breastfed for longer (Adhvaryu \& Nyshadham, 2016).

Similarly, the programme that supplied nutrient pills for mothers of infants in Ghana, aimed to improve child health. Unintendedly, the results indicated that the programme might also increase household food expenditure as well as the father's labour supply. This is consistent with the notion that fathers tend to work more to accommodate the increase in food expenditure. Although the increase in household food consumption can benefit untargeted children, the spill over effects on children's height are significant only among those with taller mothers (Adams et al., 2018). 
Second, the spill over effects of health interventions might happen through raising parents' awareness of health. They might realize that additional health inputs can improve child outcomes (learning effect). I argue that the income effect alone might be insufficient for the spill over effects to occur without the improvement in child health. For instance, the provision of a free lunch for school children in Burkina Faso tended to have no impact on the weightfor-age measure of pre-school siblings at home. Although the free lunch can relax the household budget, there was no evidence of reallocation of resources that benefit untargeted children (Kazianga et al., 2014).

Since the programme lasted for one year, I argue that parents might not realize any improvement in child outcomes from the free lunch. This could affect their decision to invest in pre-school siblings. In contrast, the results showed that parents could perceive improvements in child outcomes from iodine supplementation in Tanzania as well as nutrient pills in Ghana (Adams et al., 2018). These realized benefits might encourage them to allocate more resources to other children.

Third, the spill over effects are because of sharing. On the one hand, parents might share additional health inputs among children within a household. For instance, the school meal programme in Burkina Faso might improve the weight-for-age measure of pre-school children if children could bring home food (Kazianga et al., 2014). In this case, suppose the household budget was unaffected. Parents could share the ingredients so that household members could get some benefits. Therefore, the total effect of health inputs can be observable among children. In reality, parents can adjust the household budget. Even if the additional health inputs are indivisible, spill over effects can still occur so long as parents adjust the budget. However, if the household budget is fixed, the positive spill over effects can also happen if parents decide to cut other expenditure.

There is not much evidence on the spill over effects of free health insurance, an indivisible health input. Fitzpatrick and Thornton (2018) investigated the spill over effects of parental participation in voluntary health insurance on the healthcare utilization of children in Nicaragua. The study used a randomized trial programme that assigned insurance premiums to parents of children younger than 12 . The study found that insurance coverage might increase the healthcare utilization of young children with insured parents. Health insurance 
can lower healthcare prices, thereby increasing the demand for healthcare among the insured. This finding might suggest reallocation of resources within households can happen as healthcare prices change.

Kino et al. (2018) investigated the spill over benefits of the Affordable Care Act through a Medicaid expansion of the capacity to pay housing rent or mortgage and the purchase of nutritious meals. The 2015 study used a dataset of individuals aged 18 to 64 across 12 states in the U.S. The treatment variable was access to health insurance which was implements by expansion of Medicaid. The study used a two-stage least squares regression with IV to explain the self-reported condition of stress or worry about having enough money to pay for rent and nutritious meals.

The estimation models relied on the assumption that the ability to pay rent and buy meals was uncorrelated with the Medicaid expansion. The findings showed that access to health insurance might reduce the stress of rent payment and the purchase of nutritious food among low-income individuals. Among individuals with income below 138 percent of the Federal Poverty Line (FPL), a 10-percentage-point increase in health insurance rate was associated with a reduction in the probability of being worried about paying for housing and food by 8.6 and 7.2 percentage points, respectively. The results show no effects of health insurance on outcomes of those whose income was above 138 percent of the FPL.

Sheu and Lu (2014) estimated the spill over effect of the Taiwan Nation Health Insurance (NHI) policy started in 1995 on the pattern of household consumption. Since the NHI can provide financial protection for insured households, the study investigated how households reallocate the potential freed-up resources because of insurance. The difference-in-differences model was applied to a dataset of 17,899 households from the cross-sectional Taiwan Survey of Family Income and Expenditure surveys from 1993 to 2000. The DD model compared the changes in the proportion of consumption expenditure on medical items and non-medical items of households in the treated group (eligible to enter $\mathrm{NHI}$ ) to the changes in outcomes of the control groups before and after the policy was implemented in 1995 . The results indicate that the $\mathrm{NHI}$ had the largest positive effects on spending on housing such as rent and water bills, accounting for 1.87 percent of total household consumption. The findings also 
showed that low-income households experienced the largest increase in expenditure on rental and water bills, 2.6 percent.

\subsection{Empirical research on the impacts of SHI in Vietnam}

In this section, I discuss the impact of different health insurance policies in Vietnam. Started in 1992, the SHI policy in Vietnam has been gradually expanded to various segments of the population under either the compulsory or voluntary schemes. A. Wagstaff and Pradhan (2005) estimated the impacts of the voluntary health insurance (VHI) scheme on healthcare use, health status and household non-health expenditure. Using a panel dataset from VLSS 1993-1998 (collected before and after the introduction of VHI) and a DD model with propensity score matching, the authors aimed to eliminate any confounding effects because of selection on time-invariant unobservables. They found evidence of a positive impact of insurance on height-for-age and weight-for-age among young schoolchildren, and on body mass index among adults. The results also showed that VHI can increase outpatient and inpatient use while decreasing out-of-pocket health payments. Interestingly, this study showed that VHI can also increase household food and non-food consumption. These findings are consistent with the notion that households are not willing to increase consumption in the absence of health insurance that makes them vulnerable to large out-of-pocket expenditure. This saving was critical to households in Vietnam during the 1990s when the average cost of a single hospital visit equalled up to 20 percent of an individual's annual non-food expenditure.

Under the elementary education law of 1991, children in Vietnam must attend elementary school at age six. Since 1994, schoolchildren could buy health insurance under the Voluntary Health Insurance Scheme for Students. They could also buy private health insurance, but voluntary health insurance was still the primary source. Guindon (2014) studied the impact of this policy on the use of healthcare services in Vietnam. The author used the Vietnam Household Living Standard Surveys rounds of 2002, 2004, and 2006 and a Difference-inDifferences approach. The study showed that health insurance for students had a positive effect on inpatient visits, but not outpatient care. Given that the voluntary health insurance for student's status is likely an endogenous variable, Guindon (2014) argues that the selection bias problem should occur at the school level, not at the individual level because of the 
schooling effect on health insurance status. Even though there can be peer effects at school when parents decide to buy health insurance at school for their children, there is still no study that confirms this.

In the same line, Nguyen (2016) examined the impact of voluntary student health insurance on Vietnamese children aged six to 14. The author used fixed-effects models on two samples of VHLSS 2006-2008 and 2010-2012. Nguyen (2016) argued that individual fixed-effect estimation could solve the selection bias of voluntary health insurance. However, the fixedeffects estimation can only absorb unobserved time-invariant variables. Therefore, Nguyen (2016) investigated the potential source of endogeneity bias caused by adverse selection. The main concern was that parents of unhealthy children were more likely to buy health insurance, thereby using more healthcare services. Thus, he used a Probit regression model to explore whether previous health status affected current health insurance status. The results show no evidence of such self-selection into the insurance programme.

Various policy changes from 1993 to 1998 could hinder the effects of SHI in Vietnam. First, deregulation of the pharmaceutical industry could partly contribute to a 30 percent decrease in drug prices. However, after implementing the hospital autonomy policy, hospital fees increased by 1,000 percent and the cost of seeking care at private clinics rose by 600 percent in the period (Wagstaff \& van Doorslaer, 2001). Evidence from a quasi-experimental study showed that the policy might induce higher out-of-pocket payments for inpatient care and higher expenditure per treatment visit of 20 percent (Wagstaff \& Bales, 2012). In 1998, though only 12 percent of the population was enrolled in SHI, 80 percent of total health expenditure in Vietnam was private payments (Wagstaff \& van Doorslaer, 2003). Thus, households in Vietnam are vulnerable to health shocks. A. Wagstaff (2007b) found that the death or hospitalization of a working family member and a substantial decrease in the body mass index of the household head had negative impacts on household total income and food consumption, especially for urban households. The author also showed that the effects of hospitalization on healthcare expenditure were more pronounced for the uninsured than the insured. Similarly, using household data from 2004 to 2008, Mitra, Palmer, Mont, and Groce (2016) found that health insurance in Vietnam can provide financial protection to the insured facing health shocks. The results suggest that the negative impact of health shocks is lessened in the country over time as households could maintain their consumption. The authors argued 
that households, particularly in rural areas, coped with the shocks by borrowing, selling assets or decreasing education expenditure.

Previous studies found that, facing health shocks, low-income households are the most vulnerable. Although there was a remarkable reduction in poverty because of the economic boom of the 1990s, not all households in Vietnam reaped equal benefit. Households with a higher level of education, living in urban areas (especially those in the Red River Delta and the south-east regions), and having a white-collar job could successfully escape poverty. In contrast, low-income households often in rural areas doing agricultural jobs and with a lower education level tended to remain in destitution (Glewwe, Gragnolati, \& Zaman, 2000). One of the main sources of health financing among poor households in a rural province was loans with high interest rates from private lenders. These households tended to cut food consumption when facing health shocks. The reduction in food consumption, combined with the distress caused by the illness and debt can exacerbate the ability to work and the health status of the poor.

To protect this vulnerable segment of the population, since 2002, the government has subsidized the health insurance premium for the poor. Health Care Fund for the Poor (HCFP) relies on general revenue to finance healthcare for the poor, ethnic groups living in the Northern Midland and Mountainous areas, and households in disadvantaged communes across the country. Using a single difference model and propensity score matching and data from VHLSS 2004, Wagstaff (2007a) showed that the policy might increase healthcare utilization while decreasing the likelihood of catastrophic out-of-pocket expenditure. However, the results indicated no impact on private spending even among poor households with insurance, which had to pay a considerable amount of their income for treatment. Also, the policy might encourage the insured to use more inpatient services than outpatient services. This can further increase healthcare costs for the poor because they need to pay for the costs of transport, accommodation, etc., while receiving treatment. More importantly, the author found that the policy impact on healthcare use was more pronounced on the wealthier than the poorest households. Guindon (2014), with a double difference model and a dataset from 2002 to 2006, confirmed that the health insurance policy for the poor had positive effects on inpatient visits. 
A more recent study indicated that after 10 years of implementation, empirical studies showed mixed results for the impact of the health insurance policy for the poor. In a 2013 cross-sectional study conducted in Hanoi, Vietnam's capital, poor households with a member contracting a non-communicable disease were more susceptible to catastrophic health spending and impoverishment than those without the disease (Kien et al., 2016). This study found that health insurance coverage was unrelated to the likelihood of catastrophic health expenditure and impoverishment. The authors attributed the lack of health insurance impact to the increase in drug prices in the country during the past 10 years (Ministry of Health of Vietnam, 2014). They also argued that doctors tended to prescribe more expensive drugs that are not covered by the insurance benefit package.

In contrast, Van Minh, Kim Phuong, Saksena, James, and Xu (2013) found that SHI had a modest impact on household healthcare expenditure. Using a single difference model and a dataset from VHLSS 2002-2010, the study showed that insurance coverage might prevent poor households from incurring large health spending and falling into poverty. It should be noted that this study investigated whether a family member reporting with health insurance had any impact on household healthcare expenditure. Since health insurance cannot be shared among family members, a healthy insured person should play no role in household health payments. I argue that this study might overstate the impact of health insurance. These studies on the impact of $\mathrm{SHI}$ in Vietnam solely relied on a single comparison between households with and without health insurance. The authors cannot address the selection bias inherent in voluntary health insurance.

A similar study was conducted to examine the link between health insurance and healthcare use and spending among individuals in the mountainous areas of Vietnam. Using a sample of over 2,000 individuals aged above 18 who reported with non-communicable diseases (NCDs), Nguyen, Tran, Khuong, Phan, and Jayasuriya (2020) found that health insurance is positively correlated with healthcare use among individuals with NCDs. This can promote equity in access to healthcare for ethnic groups living in disadvantaged areas of Vietnam. However, since the results showed no link between insurance coverage and healthcare spending, this means that SHI seems to fail to protect the insured from the financial burden of illness. 
These studies focusing on the impact of health insurance policy for the poor enacted in 2002 showed differential effects of the policy across rural and urban areas as well as regions. These results motivate the examination of whether free health insurance for young children matter differentially across regions. Since Vietnam comprises of three unique regions where people have different cultures, traditions, and beliefs, I argue it is necessary to take into account the role of regional factors that could be obscured by using country-level pooled data. The findings are also relevant to central and local policy makers, and can inform local-level amendments to current policies.

In 2003, the government promoted voluntary health insurance for the whole household. This policy aimed to cover family members, farmers and homemakers who tended not to enrol in SHI. To participate in this programme, one must buy health insurance for everyone in the household. This can indirectly affect the health insurance rates of children. However, this policy has not been successful because of low numbers of participants in this health insurance scheme in 2007 and 2011 (Giang, 2008; Van Tien, Phuong, Mathauer, \& Phuong, 2011).

Though the Vietnamese government has tried to draw as many individuals as possible into the SHI pool, voluntary health insurance seems to be undesirable to the uninsured. In a cluster randomised control experiment on 3,000 households from 20 communes in Vietnam, participants were randomly selected into a control group or assigned to three treatments: a booklet with information on SHI policies and their benefits; a voucher offering a 25 percent discount on the premium; and both the information booklet and the voucher. Though the treatments seemed to be more attractive among people with current health issues, the results indicated that providing information and a premium subsidy appeared to be ineffective in expanding VHI enrolment. Similarly, it showed that information campaigns on the benefits of SHI were unsuccessful in expanding $\mathrm{VHI}$ coverage among workers in the informal sectors (only 11 percent of 23 million workers had insurance in 2008) (Wagstaff, Nguyen, Dao, \& Bales, 2016).

Enrolment in VHI was also low among young children although they are vulnerable to illness. Acknowledging this problem, since 2005 the Vietnamese government has implemented a free health insurance policy using general revenue to subsidize the premium for children under six. Palmer et al. (2014) studied the impact of this free health insurance mandate on 
healthcare utilization and the cost for children under age six in Vietnam. Using a Regression Discontinuity (RD) model, the authors compared the health-related outcomes of children whose ages are around the cut-off point (age six). They found evidence of a positive impact of such a policy on the extensive and intensive margin of utilization for both outpatient and inpatient services, but no significant effect on spending per visit.

The RD approach relies on the assumption that no other health insurance policies impact children aged six and older (the comparison group). Since these children can purchase the health insurance for schoolchildren, Nguyen et al. (2020) argued that the two different health insurance plans can have a differential impact on the outcomes of children in the treated and comparison groups. To overcome this challenge, the author used difference-in-discontinuities and triple-difference models and VHLSS 2004-2006 data to estimate the impacts of FHI for young children. The results indicated that the coverage expansion had negligible effect on healthcare use and spending as well as the health status of young children. The study suggested that the beneficiaries tended not to use FHI but seek alternative care with a higher price and quality. This feature is consistent with previous findings that show a negative correlation between income, education and the likelihood of using health insurance when seeking care in Vietnam (Sepehri, Sarma, \& Serieux, 2009). Indeed, existing evidence showed that parents chose not to use $\mathrm{FHI}$ and paid higher prices when seeking care for their young children.

In the same vein, Guindon (2014) examined the impact of free health insurance for children under six on the intensive margin of utilization for inpatient and outpatient services. The author uses a DD model to estimate the treatment effect of the policy using children aged seven to ten as the control group. The results indicate that the policy only increases the number of inpatient visits.

The inconsistent results of these studies may be a result of using surveys from different years and different estimation approaches. Palmer et al. (2014) used the Vietnam Household Living Standard Survey of 2002, 2004 and 2006 whereas Guindon (2014) used the surveys of 2006, 2008 and 2010. I argue that using children aged seven as a part of the control group may introduce bias to the estimates. Since the free health insurance policy was first implemented in 2005 , seven-year-old children may have been exposed to the treatment at a younger age. 
$\mathrm{Vu}$ (2019) explored the long-term effects of providing free health insurance for young children in Vietnam on future healthcare use. The paper found that childhood exposure to free health insurance reduced the likelihood of hospitalization but had no effect on outpatient utilization. The author also suggested that the impacts of this policy change were more pronounced on households with low-income, a low level of education and those living in disaster-prone areas.

These studies show that, after years of implementation, the FHI policy for young children is still unable to reduce out-of-pocket spending for its beneficiaries. One reason that this policy cannot provide financial protection is that children tend not to use free insurance when seeking care. In a case study on treatment for diarrhoea among young children in Ho Chi Minh city, Shieh et al. (2013) found that 29 percent of 472 inpatient cases did not use free health insurance. Parents who chose not to use FHI were more likely to use a private hospital bed for their children, which is costly. The authors suggested that these parents rather paid more than bear the longer waiting times and complicated paperwork to use $\mathrm{FHI}$.

Another explanation for the increase in healthcare expenditure on young children is that the insured tended to bypass lower-level health facilities without referral; thereby they have to pay co-insurance rates. In a case study on treatment for pneumonia and meningitis in children in Hanoi, parents directly brought their children to tertiary hospitals and were willing to pay higher user fees (Le et al., 2014). This practice is common in Vietnam since lower-level facilities are perceived as not able to deliver good quality care (Nguyen et al., 2020). It should be noted that these studies in Vietnam's two wealthiest cities where accessing health facilities is much easier than in rural parts of the country. Rural children are unlikely to have the luxury of bypassing primary facilities because of the financial burden of hospital fees as well as nonmedical costs for transport, accommodation and the forgone income while seeking care.

This thesis aims to contribute to the ongoing debate on the impact of SHI in Vietnam. Existing studies provide only a fragmented picture of the impact of various SHI policies in the country on certain individuals and household outcomes. Most focus on the direct impact of the policies on beneficiaries and the studies focus on the effects in a short time period; this overlooks a potential lag in an insurance policy. Some of the studies are plagued with the selection bias problem inherent in VHI. 
To fill these gaps, I aim to provide a broader evaluation of SHI in Vietnam using a specific insurance scheme: the FHI for children under six. I use household microdata that can capture the remarkable economic development in the country from 2002 to 2016 , a longer period than previous studies. Not only does this study investigate the policy's direct effects on the outcomes for young children, but it also focusses on the persistent effects on children who were exposed to $\mathrm{FHI}$ at a young age and the spill over effects on untargeted older children in the household and the whole household. Compared with previous studies, I examine the policy impacts on a richer set of individual health and educational outcomes as well as household indicators such as wage income, total income, living standards, health and education expenditure, health insurance coverage and calorie consumption.

This study is immune from the selection bias of VHI because the policy has been implemented nationwide so that children are entitle to $\mathrm{FHI}$ regardless of their living location or socioeconomic status. To the best of our knowledge, this study is the first to scrutinize the various effects of FHI policy using an intensive dataset. It thereby provides a broader policy evaluation that can support decision making in Vietnam and other developing countries towards universal health coverage. 


\section{Chapter 4 DATA AND ESTIMATION MODELS}

This chapter presents the research design, dataset and econometric models used in the study to evaluate the different impacts of a health insurance policy change in Vietnam. The first section describes a quasi-experimental study design in which the free health insurance (FHI ${ }^{4}$ policy for the country's young children serves as a natural experiment. Since this research design requires a dataset that was collected before and after the policy change, our study relies on multiple national representative household surveys. The plausible study design and data availability allow us to identify the causal effects of the policy change on the various outcomes of interest.

\subsection{Free health insurance policy for young children in Vietnam}

Compared to other East Asian countries with the same stage of development, Vietnamese households were more likely to experience catastrophic health expenditure that could drive them into poverty (van Doorslaer et al., 2006, 2007). In order to reduce private spending on healthcare, in 2005 Vietnam's government introduced universal health insurance that comprises of a special policy that provides free health insurance for children under six years. This policy provides children under the age of six hospital treatment, laboratory tests, and generic medicines at registered public health facilities with no cost. The government issues a health insurance card to these children where they can present it at public hospitals (where they register as primary healthcare provider) to receive free care. This gatekeeper design ensures that the insured can only access central hospitals with a formal referral from a primary care provider. It should be noted that parents who have health insurance cards for their children can still bypass the primary facilities and seek care at central hospitals with higher fees. In reality, parents often choose to forgo the free care at local hospitals due to their below-standard conditions (Trivedi, 2002). Moreover, the benefit packages are the same across different social health insurance schemes. For example, young children who are enrolled in the free health insurance programme are entitled to the same benefits as those enrolled in the health insurance programme for students. Compared to China where the

\footnotetext{
${ }^{4} \mathrm{FHI}$ in this context refers to full subsidization using general revenue for the health insurance premium for Vietnamese children under-six.
} 
amount of premium determines the level of benefits the insured can receive, it is likely that Vietnam is trying allieviate inequality in income-based healthcare access. This model might benefit the disadvantaged segment of the population while limiting the choices of care among those who are willing to pay more for better options. For instance, people who seek care using social health insurance are all subject to the overcrowded public hospitals regardless to their income. Therefore, if there were a program that could offer the insureds hospital rooms with fewer beds while charging them a higher premium, wealthier individuals may be more likely to join. This is why in Vietnam some affluent individuals can have both private and public health insurance.

\subsection{Study design}

I rely on a quasi-experimental approach to estimate the impact of the FHI policy for Vietnam's young children. In this section, I discuss the choice of the control group and the relevance of the Difference-in-Differences model.

Relying on Wagstaff (2010), I start with the basic situation that involves comparing the outcomes of insured and uninsured children. A child either has insurance or (s)he does not. If the child is insured, I observe his/her treatments. Otherwise, I observe his/her outcome without the treatment. I assume that these outcomes are captured by the following specifications:

$$
\begin{gathered}
y^{0}=k_{0}(X)+\alpha+\varepsilon=k_{0}(X)+u_{0}(1) \\
y^{1}=k_{1}(X)+\alpha+\beta+\varepsilon=k_{1}(X)+u_{1}(2)
\end{gathered}
$$

where: $y^{0}$ and $y^{1}$ are the without insurance and with insurance outcomes of a given child; $k_{0}(X)$ and $k_{1}(X)$ are the functions to capture the effects of a group of control variables, $X$, on the outcomes; $\alpha$ is a constant term that represents the effects of unobserved characteristics of the child on the outcomes; $\beta$ is the unobserved impact of insurance on the outcomes for the insured child; and $\varepsilon$ is the error term. The effect of insurance on the insured child is the difference between the with and without treatment outcomes:

$$
y^{1}-y^{0}=k_{1}(X)-k_{0}(X)+\beta=g(X)+\beta(3)
$$


where: $g(X)$ is the effect of the observed individual characteristics; and $\beta$ is the unobserved impact of the policy change on insured children.

To evaluate the effect of insurance coverage, one can ask what the outcome would have been for insured children had they not been covered. In this case, the purpose is to estimate the impact of insurance on treated children. Similarly, one can ask what the outcome would have been for those who were uninsured if they had been given the treatment. This is equivalent to measuring the effect insurance would have had on untreated children. Thus, the conditioning on the observed characteristics, $X$, the average treatment effect on the treated (ATT) and the average treatment effect on the untreated children (ATU) can be estimated by:

$$
\begin{aligned}
& A T T(X)=E\left[y^{1}-y^{0} \mid X, T=1\right]=g(X)+E[\beta \mid T=1](4) \\
& \operatorname{ATU}(X)=E\left[y^{1}-y^{0} \mid X, T=0\right]=g(X)+E[\beta \mid T=0](5)
\end{aligned}
$$

These effects will differ from one another if the treated and untreated children differ in the observed characteristics, $X$ (e.g., it can be easier for children in an urban area to use health insurance than those in a rural area), and if the impact of the unobserved effect of insurance differs between these children (e.g., children who are vulnerable to illness are more likely to purchase health insurance). The policy change that our study relies on provided FHI for young children across the country, thereby ruling out this self-selection issue. Since I aim to examine how successful the FHI policy has been to date, I am interested in estimating the ATT. If the objective were to investigate how older children, who are currently not entitled to the policy, would benefit from receiving FHI, ATU is the relevant estimate. Both ATT and ATU are information for policymakers. In the following sections, I present the common methods to estimate ATT and ATU.

\section{The drawback of single difference}

The simplest approach to infer ATT and ATU is to compare the outcomes for children with and without health insurance. To illustrate the problems that might arise in using this method, I use potential outcomes for the treated and untreated groups of children. First, the expected outcomes for those who have insurance are as follows:

$$
E\left[y^{1} \mid X, T=1\right]=g_{1}(X)+E[\alpha \mid X, T=1]+E[\beta \mid X, T=1]+E[\varepsilon \mid X, T=1]
$$




$$
E\left[y^{0} \mid X, T=1\right]=g_{0}(X)+E[\alpha \mid X, T=1]+E[\varepsilon \mid X, T=1]
$$

Equation (6) represents the observed outcomes for insured children; equation (7) is the unobserved hypothetical outcome of these insured children had they not been covered. Similarly, I can set up the potential outcomes for the untreated children:

$$
\begin{gathered}
E\left[y^{0} \mid X, T=0\right]=g_{0}(X)+E[\alpha \mid X, T=0]+E[\varepsilon \mid X, T=0] \\
E\left[y^{1} \mid X, T=0\right]=g_{1}(X)+E[\alpha \mid X, T=0]+E[\beta \mid X, T=0]+E[\varepsilon \mid X, T=0]
\end{gathered}
$$

where the observed outcome for children without insurance is generated by equation (8) and the unobserved hypothetical outcomes for these untreated children had they been covered is captured by equation (9).

To estimate the ATT, I could compare the observed outcome of treated children in equation (6) to the counterfactual outcome of these children in equation (7). Since this hypothetical outcome is unobserved, the single difference involves comparing the outcome of equation (6) with the observed outcome of the untreated children in equation (8). However, the outcomes in equations (7) and (8) are systematically different regarding $\alpha$ and $\varepsilon$. In other words, this comparision is problematic because children with and without insurance can differ in unobserved characteristics. Therefore, the single difference in the expected outcomes between the insured and uninsured can be specified as:

$$
E[y \mid X, T=1]-E[y \mid X, T=0]=A T T(X)+E[\alpha \mid X, T=1]-E[\alpha \mid X, T=0]
$$

Therefore, the single difference with conditioning on the $X^{\prime} s$ might provide a biased estimate of the effect of insurance because insured and insured children differ in their unobservable $\alpha$ and $\varepsilon$. It should be noted that treated and untreated children can also differ in unobserved characteristics that make them benefit more or less from having insurance (e.g., insurance can be more beneficial to vulnerable young children than to older children). However, as can be seen in (10), the difference in the $\beta \mathrm{s}$ does not bias the ATT estimate.

To estimate ATU, one would compare the observed outcome of children without insurance in equation (8) to the counterfactual outcome of these children in equation (9). Again, the outcome in (9) cannot be observed. Instead, I can compare the expected outcomes of the 
untreated children in (8) to treated children in (6). However, the outcomes of those in equations (6) and (9) systematically differ in their $\alpha, \varepsilon$, and $\beta$. Thus, the difference in mean outcome between the treated and untreated children in this case can be decomposed as:

$$
\begin{gathered}
E[y \mid X, T=1]-E[y \mid X, T=0]=A T U(X)+E[\alpha \mid X, T=1]-E[\alpha \mid X, T=0]+ \\
E[\beta \mid X, T=1]-E[\beta \mid X, T=0]
\end{gathered}
$$

Generally, the single difference approach is useful in randomised control trials (RCT) in which health insurance is randomly assigned to children. For example, in a large-scale experiment in which health insurance premiums were subsidized (the RAND project started in 1971), insured individuals indeed increased healthcare utilization and spent more for healthcare (Aron-Dine, Einav, \& Finkelstein, 2013; Brook et al., 1984). The Oregon Health Insurance Experiment in 2008 randomly provided uninsured individuals a chance to apply for Medicaid. The results from that randomized control experiment showed that health insurance statistically significantly increased health utilization, lowered health expenses, and improve physical and mental health. Conditioning on the observed characteristics, $X$, the simple comparison of the outcomes of insured and uninsured children can produce an unbiased average treatment effect of insurance since ATT and ATU are equal in the context of an RCT.

Since conducting such an expensive experiment is often financially infeasible, many studies have relied on a quasi-experimental research design to estimate the effects of health insurance. The quasi-experimental design relies on non-experimental data to estimate the causal effects of health insurance. This approach exploits an abrupt change in policy that affects many, but not all, individuals at the same time. The primary purpose is to mimic an ideal experiment (Angrist \& Pischke, 2008).

However, with cross-sectional data, the simple difference between children with and without insurance tends to produce a biased estimator of the impact of insurance. As is pointed out, the estimator of ATT is biased because of either the difference in observable characteristics $\mathrm{X}^{\prime}$ 's or the difference in unobservables $\alpha$ and $\varepsilon$. There is also another source of bias related to the unobserved marginal impact, $\beta$, of having insurance. Since our study evaluates how successful the FHI policy for young children in Vietnam has been to date, ATT is the relevant estimator of interest. Because the policy has been implemented nationally, every under-six 
child is entitled to FHI. As a result, I can only choose older children who are not eligible for the policy as the comparison group. Since young and older children can differ in key unobserved characteristics, such as vulnerability to illness, that can affect the outcomes, using the single difference will produce a biased ATT in this case.

\section{Difference-in-Differences approach}

I argue that a DD model can eliminate or reduce the bias because of unobservables that cannot be solved with the simple difference. With multi-year microdata, I can observe the outcome of treated children aged under six at time $t_{1}$, before the policy changed and the outcome for similar-aged children at time $\mathrm{t}_{2}$, after they can receive FHI. Thus, I can decompose the mean difference in equation (10) as:

$$
\begin{gathered}
E\left[y \mid X, T=1, t=t_{2}\right]-E\left[y \mid X, T=0, t=t_{2}\right]=\operatorname{ATT}(X)+E\left[\alpha \mid X, T=1, t=t_{2}\right]- \\
E\left[\alpha \mid X, T=0, t=t_{2}\right](12)
\end{gathered}
$$

None of the treated and untreated children could receive $\mathrm{FHI}$ at time $\mathrm{t}_{1}$. Thus, the expected difference in outcome between the two groups of children at time $t_{1}$ is as follows:

$$
\begin{gathered}
E\left[y \mid X, T=1, t=t_{1}\right]-E\left[y \mid X, T=0, t=t_{1}\right]=E\left[\alpha \mid X, T=1, t=t_{1}\right]- \\
E\left[\alpha \mid X, T=0, t=t_{1}\right](13)
\end{gathered}
$$

I subtract Equation (13) from Equation (12) to measure the DD estimator of ATT that indicates the change in the difference in expected outcomes between the treated children (aged under six) and the untreated children (aged six to 15) over time:

$$
\begin{gathered}
\left\{E\left[y \mid X, T=1, t=t_{2}\right]-E\left[y \mid X, T=0, t=t_{2}\right]\right\}-\left\{E\left[y \mid X, T=1, t=t_{1}\right]-\right. \\
\left.E\left[y \mid X, T=0, t=t_{1}\right]\right\}=A T T(X)+\left\{E\left[\alpha \mid X, T=1, t=t_{2}\right]-E\left[\alpha \mid X, T=1, t=t_{1}\right]\right\}- \\
\left\{E\left[\alpha \mid X, T=0, t=t_{2}\right]-E\left[\alpha \mid X, T=0, t=t_{1}\right]\right\}
\end{gathered}
$$

The DD estimator in Equation (14) relies on the parallel trend assumption that requires the outcomes of treated and untreated children to follow the same pattern in the absence of treatment. In other words, this assumption ensures that the last two components in Equation (14) are the same so that the DD estimate equals ATT(X). 
It is noteworthy that the parallel trend assumption is weaker than the assumption of a timeinvariant $\alpha_{\mathrm{i}}$ that underpins the fixed effect models with panel-data. The DD model requires no assumption about the marginal impact of insurance $\beta_{\text {i }}$ since it is not the source of bias in ATT. Though it is unclear whether I have a biased estimate of ATT or ATU under the single difference approach, the DD model provides an unambiguous estimate of ATT.

This study relies on Vietnam's FHI policy change. Since 2005, children under six across the nation can receive free health insurance. This policy can create an exogenous variation in health insurance coverage across an age cohort and year. I account for unobserved factors at age and year level using various control groups.

Particularly, this study estimates the causal effects of free health insurance on children and their households using the following control groups. First, to retrieve the direct effects of this policy on children under six, I use a control group of children aged six to ten. This group controls for the changes over time in the country. I also control for potential age differences in the health outcomes of these children. The data show that these children have similar patterns of morbidity. It should be noted that our control group of children aged six to ten were observed from different surveys conducted in different years. Therefore, some children in the control group were exposed to $\mathrm{FHI}$ at a young age (for example, older children in the survey 2010 have longer exposure to $\mathrm{FHI}$ at a younger age than those observed in 2006). If the policy had indeed improved the outcomes of the children in the control group, the impact of $\mathrm{FHI}$ on young children may be underestimated. I could alleviate this issue by including variables indicating the likelihood and intensity of previous exposure using information from year of birth and survey. However, this will cause multicollinearity issues because I control for survey year and age in our estimation model. Informing this issue, however, I also investigate the persistent effect of $\mathrm{FHI}$ on older children, the outcomes of which can give some indication as to whether the direct effects are downwardly biased.

Second, to measure the persistent effects on children who had previous exposure to free health insurance at a young age, I compare the outcomes for children between the age cohorts. For example, differences in outcomes for six-year-olds before and after the policy change can be because of previous exposure to free health insurance at a young age and time effects. To isolate the time trend, I use a control group of older children who had no exposure. 
Third, to estimate the spill over effects on children who live with young siblings, I compare the outcomes of these children before and after the policy change. I capture the time trend by using a control group of similar-age children who do not have younger siblings. Since parents made fertility decisions before the policy change, the presence of young children in households is exogenous. Similarly, I compare the outcomes of households with young children observed before and after the intervention. The difference in outcomes of households without young children over time can be used to control for year effects.

Under this framework of a quasi-experimental research design, I use DD regression models and data from national household surveys to measure the effects of free health insurance for young children on household and individuals.

\subsection{Data}

This study estimates the causal effects of health insurance using a DD model. This estimation model requires a dataset of children and households observed before and after the policy change in 2005. I use the Vietnam Household Living Standard Surveys from 2002 to 2016. Since the health insurance policy might take a long time to have a significant impact on health outcomes (Giedion, Alfonso, \& Díaz, 2013), unlike previous studies in Vietnam that focussed on a shorter time frame, this study uses a dataset spanning 14 years. In the following parts, I discuss the survey's design and questionnaires, as well as the construction of variables needed for analysis.

\subsubsection{Vietnam Household Living Standard Surveys}

To measure the impact of free health insurance for Vietnamese children under six, I use data from the Vietnam Household Living Standard Surveys (VHLSS). VHLSS, conducted every two years since 2002, was designed to collect data on the standard of living to assist in planning public policy and the socio-economic development strategy. I use eight rounds of VHLSSs from 2002 to 2016 that provide individual and household-level data on demographic characteristics, migration, health and education, employment and income, expenditure, housing conditions, and participation in poverty reduction programmes. 
The design

Before the VHLSS, there were two rounds of a standard living survey (VLSS) in 1993 and 1998. Compared with those surveys, VHLSS has larger sample that contains over 3,000 communes, accounting for approximately 30 percent of total communes in the country. In each commune, three households are chosen to create a sample of over 9,000 households. The selection of households is based on a stratified random sampling procedure. It should be noted that there was a change in sampling frame of VHLSS between 2002 and 2016. The 20022008 sampling frame was based on the 1999 Housing and Population census. According to that census, about $80 \%$ of households in Vietnam lived in rural areas. Therefore, the selection of households in the VHLSS was based on stratification to guarantee that that sample was $80 \%$ rural households.

The 2002-2008 sampling procedure of VHLSS followed two steps. The first step involved creating a master sample from the Housing and Population census in 1999. In that census, there were 61 provinces and 10,475 communes in Vietnam (Hansen \& Le, 2013). The communes were then categorized into urban and rural areas. The master sample was created by selecting the communes from these urban and rural lists by province. This approach ensured that the communes were distributed evenly across the country. As a result, 3,000 communes were selected with the probability proportional to the number of households in the communes. Next, three enumeration areas (EAs) in each commune in the master sample were drawn with the probability proportional to the number of households in the EAs. Consequently, the master sample contained over 3,000 communes and 9,000 EAs.

The second step was to create a VHLSS sample of households from the master sample. Specifically, 1,500 EAs were selected from the 9,000 EAs in the master sample, with the probability proportional to the number of households in the EAs. It should be noted that $80 \%$ of the selected EAs were from rural areas for each province across the country. Next, a certain number of households was randomly selected in each EA. In VHLSS 2002, 20 households in each EAs were selected to participate in the survey of household income and expenditure. However, in later rounds of VHLSSs, only three households were interviewed, so the number of households in these surveys was over 9,000 compared with about 30,000 in VHLSS 2002. It should also be noted that, in later VHLSS rounds, half of the households that were 
interviewed in previous surveys were reselected and half the households were new from the EAs in the master sample. This study does not exploit the longitudinal element of VHLSS and solely focussed on creating a repeated cross-sectional dataset.

VHLSS 2010-2016 was based on the sampling frame from the Housing and Population census of 2009. The master sample created from the 2009 census contains 5,490 communes (76\% from rural areas) and 16,470 EAs. From this master sample, 3,063 EAs were selected of the 16,479 in the master sample and three households were randomly selected from each of these 3,063 EAs. VHLSS 2010 is thus a sample of 9,189 households. It should be noted that more households were allocated to provinces with a small population to ensure representativeness at the provincial level.

The VHLSS was designed to interview selected household members directly. In the section on education and health, all members were encouraged to give answers. Meanwhile, in the employment and income section, members who had jobs or generate income were invited to answer. Questions about household expenditure, durables, and housing conditions were for the household head or any member who had the most information. For young children or members who were unable to respond, the household head was expected to give answers on their behalf. Generally, VHLSSs collected data at the beginning of every quarter and the reference period for most questions in the questionnaires was the last 12 months.

Table 4.1 presents the descriptive statistics of some household characteristics in the VHLSSs. As previously mentioned, since the sampling frame of VHLSS was different in 2010, the proportion of rural households has declined. The decrease in rural households from 80 percent in 2002 to 70 percent in 2016 might reflect the socio-economic development in Vietnam over that time. There were other changes in housing conditions and household composition in the period. The size of the living place had increased gradually from $56 \mathrm{~m}^{2}$ in 2002 to $84 \mathrm{~m}^{2}$ in 2016 and the household size had decreased from 4.48 to 3.81 persons, on average.

There were no differences in the presence or number of children under six in the households. There were not many changes in the characteristics of the household head over time. The chance that a household head was male and married were consistently about 75 and 80 
percent, respectively. However, in recent surveys heads were reported as being slightly older and with a better education level. Household living standards in Vietnam improved significantly over time. Compared with 2002 , household income and expenditure ${ }^{5}$ per capita in 2016 were eight and nine times higher, respectively. The percentage of household members with health insurance increased significantly, especially after the free health insurance policy for young children started in 2005.

Table 4. 1 Household Characteristics

\begin{tabular}{lrrrrrrrr}
\hline Year & 2002 & 2004 & 2006 & 2008 & 2010 & 2012 & 2014 & 2016 \\
\hline Rural & 0.77 & 0.76 & 0.75 & 0.74 & 0.72 & 0.71 & 0.70 & 0.70 \\
Kinh Ethnicity & 0.85 & 0.85 & 0.84 & 0.84 & 0.82 & 0.82 & 0.83 & 0.82 \\
Living Area & 56.15 & 60.19 & 63.09 & 68.05 & 69.9 & 74.61 & 80.62 & 84.33 \\
Household Size & 4.48 & 4.41 & 4.25 & 4.16 & 3.94 & 3.92 & 3.84 & 3.81 \\
Having children under six & 0.34 & 0.27 & 0.26 & 0.27 & 0.31 & 0.30 & 0.31 & 0.29 \\
Number of children under & & & & & & & & \\
six & 0.38 & 0.33 & 0.32 & 0.34 & 0.38 & 0.36 & 0.37 & 0.37 \\
& & & & & & & & \\
Male head & 0.76 & 0.76 & 0.75 & 0.76 & 0.75 & 0.75 & 0.74 & 0.75 \\
Head's Age & 47.55 & 49.09 & 49.36 & 49.96 & 48.35 & 49.75 & 50.74 & 51.7 \\
Being Married & 0.82 & 0.82 & 0.82 & 0.82 & 0.82 & 0.81 & 0.80 & 0.80 \\
Head's Education (Grade) & 6.65 & 6.86 & 6.97 & 7.10 & 7.14 & 7.17 & 7.30 & 7.35 \\
& & & & & & & & \\
Income Per Capita & 4.52 & 6.11 & 9.53 & 11.74 & 19.75 & 23.83 & 30.67 & 36.65 \\
Expenditures Per Capita & 3.71 & 4.89 & 6.83 & 9.48 & 18.71 & 22.38 & 29.36 & 33.37 \\
Health insurance coverage & $\mathrm{N} / \mathrm{A}$ & 0.39 & 0.54 & 0.57 & 0.61 & 0.65 & 0.70 & 0.78 \\
Number of Households & 29,533 & 9,188 & 9,189 & 9,189 & 9,402 & 9,399 & 9,399 & 9,399 \\
\hline Note: Data from Vietnam Household & & & & & & & & \\
\hline
\end{tabular}

Note: Data from Vietnam Household Living Standard Surveys 2002 to 2016

Table 4.2 presents some information on the children samples that will be used to estimate the effects of the free health insurance policy. I use a sample of children under 11 years to examine the direct effect of the policy on children under six. The proportion of under-six children was around 50 percent, and the probability of having health insurance among

\footnotetext{
${ }^{5}$ Monetary amounts in this thesis are expressed in real terms.
} 
children in this sample increased significantly by over 40 percentage points after the policy change.

Table 4. 2 Individual characteristics

\begin{tabular}{|c|c|c|c|c|c|c|c|c|}
\hline Year & 2002 & 2004 & 2006 & 2008 & 2010 & 2012 & 2014 & 2016 \\
\hline \multicolumn{9}{|c|}{ Sample 1: Children Aged Under 11} \\
\hline Sample Size & 25,889 & 6,932 & 6,042 & 5,870 & 6,608 & 6,338 & 6,406 & 6,325 \\
\hline $\begin{array}{l}\text { \% of Children } \\
\text { U6 }\end{array}$ & 0.43 & 0.44 & 0.48 & 0.53 & 0.54 & 0.53 & 0.54 & 0.54 \\
\hline Rural & 0.81 & 0.81 & 0.8 & 0.78 & 0.75 & 0.73 & 0.73 & 0.72 \\
\hline Kinh Ethnicity & 0.78 & 0.76 & 0.75 & 0.76 & 0.75 & 0.75 & 0.76 & 0.75 \\
\hline Health & N/A & 0.46 & 0.82 & 0.87 & 0.88 & 0.92 & 0.95 & 0.96 \\
\hline
\end{tabular}

Sample 2 \& 3: Children Aged Six to 15

\begin{tabular}{|c|c|c|c|c|c|c|c|c|}
\hline Sample Size & 31,860 & 9,084 & 7,800 & 6,861 & 6,279 & 6,028 & 5,827 & 5,801 \\
\hline Rural & 0.82 & 0.81 & 0.8 & 0.79 & 0.77 & 0.75 & 0.74 & 0.73 \\
\hline Kinh Ethnicity & 0.8 & 0.78 & 0.77 & 0.78 & 0.74 & 0.75 & 0.76 & 0.76 \\
\hline $\begin{array}{l}\text { Health } \\
\text { Insurance Rates }\end{array}$ & N/A & 0.63 & 0.77 & 0.81 & 0.82 & 0.88 & 0.92 & 0.95 \\
\hline $\begin{array}{l}\text { Exposure to FHI } \\
\text { policy }\end{array}$ & 0 & 0 & 0.07 & 0.23 & 0.48 & 0.7 & 0.91 & 1 \\
\hline $\begin{array}{l}\text { Living with } \\
\text { Children U6 }\end{array}$ & 0.27 & 0.27 & 0.27 & 0.28 & 0.31 & 0.3 & 0.3 & 0.31 \\
\hline Total Individuals & & & & & & & & \\
\hline $\begin{array}{l}\text { (including } \\
\text { adults) }\end{array}$ & 132,385 & 40,438 & 39,071 & 38,253 & 37,012 & 36,655 & 36,094 & 35,798 \\
\hline
\end{tabular}

Note: Data from Vietnam Household Living Standard Surveys 2002 to 2016.

I use the sample of children aged six to 15 to measure the persistent and spill over effects of the policy on these children. I notice that the health insurance rates of children older than six increased from 0.63 in 2004 to 0.77 in 2006 . This increase can be the result of past exposure to the policy or through living with young siblings who were entitled to FHI. I can see that not all children experienced $\mathrm{FHI}$ at a young age. Only 7 percent of children aged six to 15 in 2006 
were entitled to free health insurance for under-six children when they were young. Using this variation in the receipt of treatment, I later use a DD model to estimate the prolonged effects of the policy. Likewise, about 30 percent of children aged six to 15 lived with undersix-year old siblings. I later use the DD model to investigate whether the presence of young siblings in the household can affect the outcomes for older children.

\subsubsection{The questionnaires}

Although there were some changes in the questionnaire over time, the VHLSS questionnaire generally had eight parts. The first part is the cover page that provides information on household ethnicity and the living location. Section I has questions about age, gender, marital status, and relationship to household head of all household members as well as those who were temporarily away from the household. I mostly rely on age to determine the treatment status of children in the analyses. For example, I focus on children under six to examine the direct effects of FHI policy and I rely on children's age and the policy change year to investigate whether being exposed to and the duration of exposure to $\mathrm{FHI}$ had any persistent future impacts on young children. I use the presence of children under six in the household as an indicator of treatment to study the spill over effects on older children and the household. The code system for the relationship to household head in VHLSS did not allow us to identify the kin relationship among members in extended households. A new code system has been available since VHLSS 2014.

In Section II, the focus is on the education of all household members, including educational attainment, school participation and education expenditure. To estimate the effects of the FHI policy on children's education, I rely on two outcomes: school attendance in the last 12 months and education expenditure. The question on school participation was consistent across surveys, but there were some changes in the question on education expenditure. Generally, across surveys, individual education expenditure comprised tuition fees, contribution to class or school funds, uniforms, school books and other materials as well as tutoring fees. Since 2006, the education expenditure category started to include other 
expenses such as fees if children attended school out of their registered living location and other expenses for transport, housing rent and contributions to various school funds ${ }^{6}$.

Section III covers health information of all household members, including morbidity and health insurance status as well as healthcare utilization and expenditure. There were two measures of morbidity: in the last 30 days and the last 12 months. The question of morbidity in the last 30 days was available from VHLSS 2004 to 2008, and the question on last year's morbidity rate was only in surveys in 2004, 2006, 2008, 2014 and 2016. The question of morbidity in the last 12 months was modified in the 2014 and 2016 surveys to emphasize the seriousness of the illness or injuries. I argue that this change can affect the probability of reporting an illness or injury. Therefore, in my analyses, I use data on morbidity rates only in VHLSS 2004 to 2008. Although the questions on health insurance, healthcare utilization and expenditure were consistent across surveys, VHLSS 2002 did not ask household members about their insurance status nor the number of outpatient and inpatient visits.

Section IV mainly focuses on the employment and the economic activities of all household members. The employment questions were available to members older than six years old and cover employment status as well as primary and secondary jobs. The job-related questions included type, sector, the number of hours, days, and months of working, and the wage or salary of the jobs. Since my analysis examines whether free health insurance can affect household wages, I aggregate income sources from the paid jobs into a total wage of the household. The questions on other economic activities applied to the whole household. These activities include agricultural, forestry, and fishery production or processing, and business enterprises. Since VHLSS questions provide about revenue and costs of the economic activities, I can measure and aggregate the income sources from the activities with other income sources into the household income. I use this to estimate the effects of free health insurance policy on household net income per capita.

Section $\mathrm{V}$ had detailed questions on household expenditure, including daily and annual spending on food, non-food items and other expenses. Since these questions provided information on the type and quantity of food consumed, I can calculate the calories consumed

\footnotetext{
${ }^{6}$ I test the sensitivity of the results when excluding these additional costs.
} 
in households. Thus, I use caloric consumption as an outcome variable in my analysis of the impact of the FHI policy on household nutritional status.

Section VI focuses on questions about the purchase of household durables. In some VHLSS surveys, there were separate lists of durables and fixed assets. When there were no such lists, I distinguished the two kinds of property. In Section VII, there were questions about housing conditions. These questions involved the size of living areas, the structures and materials of the house. There were also questions on the expenses for housing utilities such as electricity and water supply and garbage disposal. Since I aim to estimate the effects of the FHI policy on household expenditure per capita, I explain later how I construct the aggregate of the expenditure using data from all sources on household consumption. Section 8 of VHLSSs comprises questions on participation in current poverty alleviation programmes in Vietnam.

\subsubsection{Variables}

Using VHLSS 2002 to 2016, I create three data samples to address four research questions. First, to examine whether $\mathrm{FHI}$ for children under six has direct effects on its beneficiaries, I use a sample of children aged under 11 . Specifically, I focus on the available outcomes of these children from VHLSSs, including health insurance, healthcare utilization and expenditure, as well as morbidity status. Second, my study uses a sample of children aged six to 15 to estimate the persistence and spill over effects of the policy on older children in the household. I also focus on school attendance and education expenditure of these school-age children. Third, I measure the effects of the FHI policy on households using a sample of all households in the surveys. Household outcomes include wage, income, expenditure, health insurance coverage and calorie consumption.

Although most outcome variable in this study can be extracted directly from VHLSSs, the treatment variable for persistent effect analysis and the calculation of household expenditure per capita are not straightforward. First, to estimate the prolonged effects of the FHI policy, I build a measure of the duration of exposure to the FHI policy for children aged six to 15 . Specifically, I develop the treatment intensity variable using children's age and the year when the policy changed. Table 4.3 summarizes the intensity of treatment by children's age over time. There were variations in the treatment of children within and between age cohorts. For 
example, one year after the policy change, six-year-old children in 2006 would have one more year of free health insurance compared with six-year-old children in 2004 or seven-yearchildren observed in the same year.

Table 4. 3 The duration of exposing to free health insurance by age and survey year

\begin{tabular}{crrrrrrrrrr}
\hline Year/Age & 6 & 7 & 8 & 9 & 10 & 11 & 12 & 13 & 14 & 15 \\
\hline 2002 & 0 & 0 & 0 & 0 & 0 & 0 & 0 & 0 & 0 & 0 \\
2004 & 0 & 0 & 0 & 0 & 0 & 0 & 0 & 0 & 0 & 0 \\
2006 & 1 & 0 & 0 & 0 & 0 & 0 & 0 & 0 & 0 & 0 \\
2008 & 3 & 2 & 1 & 0 & 0 & 0 & 0 & 0 & 0 & 0 \\
2010 & 5 & 4 & 3 & 2 & 1 & 0 & 0 & 0 & 0 & 0 \\
2012 & 6 & 6 & 5 & 4 & 3 & 2 & 1 & 0 & 0 & 0 \\
2014 & 6 & 6 & 6 & 6 & 5 & 4 & 3 & 2 & 1 & 0 \\
2016 & 6 & 6 & 6 & 6 & 6 & 6 & 5 & 4 & 3 & 2 \\
\hline
\end{tabular}

Note: I assigned the treatment of FHI using the information on age and the year of the policy change. Data from VHLSS 2002 to 2016.

I construct a household consumption aggregate using the guidelines of (Deaton \& Zaidi, 2002). I then combine this amount of consumption with household expenditure on healthcare and education to calculate total household expenditure. In my analysis, I study the effect of the FHI policy on household living standards measured by household expenditure per capita. Although income per capita is one outcome of the study, I argue that household expenditure as a measure of living standard is more relevant in the context of Vietnam.

Deaton and Zaidi (2002) suggest that household consumption is less likely to be affected by fluctuations in income in the short-term and that consumption is less variable than income. Since empirical evidence showed that households could smooth their consumption in the short run, the measure of living standards by consumption is more stable than using income. In Vietnam, about $80 \%$ of households living in rural areas were subject to income fluctuation from agricultural activities. Therefore, using consumption as a measure of welfare is more appropriate. Even if consumption also fluctuates with festivals or holidays, this variation in consumption can still be smaller than income fluctuations. In VHLSS, there were detailed questions on festival and daily food consumption so that I can account for part of the variation in consumption. 
Aggregate household consumption consists of four main components: food and non-food items, consumer durables, housing rent, and utilities. In VHLSSs, though the time reference for holiday food consumption was the last 12 months, daily food consumption was recorded for the last 30 days. Therefore, I aggregate daily food consumption to year level (with an adjustment for the Tet holiday of two weeks). I also convert daily non-food consumption into year level. The questions about food and non-food items were specific about the quantities purchased or self-produced and their total value.

On the other hand, instead of using the purchase value of durable goods, I add their use-value into the consumption aggregate. The use-value of consumer durables can be considered as the cost of using the durables for a certain period. In other words, I estimate the use-value of durables as if households purchased the durable good at the beginning of the year and sold it at year's end. Specifically, I use the following formula to estimate the user cost of durables presented by Deaton and Zaidi (2002). In the formula, $S_{t}$ is the number of durable goods and $P_{t}$ and $P_{t+1}$ are the purchase price at the beginning and year's end; $r_{t}$ is the nominal interest rate of holding the durables for a year, and $\delta$ is the rate of depreciation in the value of the durable. This depreciation rate is based on the assumption that the value after using it for a year is lower than the purchase value. Therefore, formula (1) is the discounted present value of the durable goods at year's end:

$$
S_{t}\left(P_{t}-P_{t+1} \frac{1-\delta}{1+r_{t}}\right)
$$

I estimate the use cost of durable goods using the following alternative formula:

$$
S_{t} P_{t}\left(r_{t}-\pi_{t}+\delta\right)
$$

where: $S_{t} P_{t}$ is the current value of durable goods; $\left(r_{t}-\pi_{t}\right)$ is the real interest rate; and $\pi_{t}=$ $\left(\frac{P_{t+1}-P_{t}}{P_{t}}\right)$ is the inflation rate of the price of durables. I calculate the depreciation rate of durable goods using the following formula:

$$
\delta-\pi=1-\left(\frac{P_{t}}{P_{t-T}}\right)^{1 / T}
$$


I avoid large variations in the price of a durable good, the use of the median of depreciation rates by year, region, and urban/rural areas.

Similarly, it would make no sense to include house purchase in the household consumption aggregate because this expenditure is rare and large. I rely on the same approach to estimate the use-value of durable goods to measure the implicit rental value of a house. To VHLSSs, I add the rental payment of households that paid the rent in the consumption aggregate. The renters were also asked to provide an estimate of the value of the house they were renting or living in. Based on this information, I calculate a ratio of rent to estimated house value and use this ratio to measure the rental payment of households that were not renters. To minimize fluctuations in housing prices, I use the mean of rental payment by year, urban/rural area and region?

\subsection{Estimation Models}

\subsubsection{Difference-in-Difference models for the direct effects of the free health insurance policy}

This study uses DD strategy to estimate the causal effect of the policy of free health care for children under six on their health insurance coverage, health utilization and spending, and morbidity rate. Children aged six to ten serve as the counterfactual for children under six. The identification strategy relies upon the assumption that the health outcomes of the two groups of children would have had the same time trend in the absence of the FHI mandate. When testing for the parallel trend assumption, it is better to have many years of data prior to the policy change because it would show whether health-related outcomes of children in the control and treatment are systematically different. Although there are four VHLSS surveys conducted before 2005, the year FHI was first implemented, the changes in questionnaires did not allow me to gauge consistent data on health outcomes such as healthcare use, except for the survey in 2004. Using only one year before to policy change to test the parallel trend

\footnotetext{
7 Alternatively, one can use regression to predict rents on housing characteristics such as household size, structure, number of rooms and the availability of access to electricity or clean water as well as location. The estimates of this regression can then be used to predict the rent payment of non-renters. However, though the number of renters in VHLSSs was small, to make a reliable prediction, the questions on housing characteristics were inconsistent. Therefore, this approach is not relevant to this study.
} 
assumption, however, would be inadequate. Fortunately, the usable information on morbidity status from survey 1992, 1998, 2004, and 2006 allowed me to examine whether children under six and six to ten have different health status. Indeed, later I test this assumption using morbidity status; there is no evidence in the tests to indicate children in the treated and control groups have different morbidity rates. In Figure 4.1, the morbidity rates of these children seem to decline at similar rates. It should be noted that I can use morbidity data from VLSS 1993-1998 and VHLSS 2004-2008 because the questions on illness were changed. The simplest DD OLS regression estimates the effect of the free health care policy on individual $i$ 's outcomes in year $t$ without controlling for observables and year fixed effects:

$$
Y_{i t}=\alpha+B_{1} \text { PostPolicy }_{t}+B_{2} \text { Under }_{i}+B_{3} \text { PostPolicy }_{t} \times \text { Under }_{i}+\varepsilon_{i t}
$$

where: $Y_{i t}$ represents the outcome of interest such as health insurance status, health utilization and medical costs, and morbidity rate of individual $i$ at year $t$; PostPolicy $t$ is a binary variable equalling one in the years after the policy implementation in 2005 ; and Under $6_{i}$ is an indicator for children under six. The coefficient $b_{3}$ captures the causal effect of the policy on the outcome variable. However, this specification may be susceptible to potential omitted variable biases from unobserved individual characteristics such as mortality rate or parents' risk preference. These confounding factors can be correlated with outcome variables and interaction term PostPolicy ${ }_{t} \times$ Under6 $_{i}$, which may bias the estimates of the impact of the policy. To account for this, I include relevant control variables for the differences in characteristics between the two groups of children. The inclusion of control variables is expected to reduce residual variance as well as gain additional efficiency for estimators. It also relaxes the assumptions necessary for identification in the DD framework, since the two groups need only follow parallel trends conditional on controls.

I introduce indicator variables for region, urban/rural, ethnicity, age, age squared, gender, and household head characteristics to equation (4.1.1). The analysis controls for the sex of children since Treleaven et al. (2016) showed evidence of gender effects of healthcare use among children in northern Vietnam. I use variable age to capture the differences in outcomes of children of different age groups. I include variable age square to reflect the possibility that the age effects may be different as children age. 
I control for the gender of the household head because existing evidence shows that females invest more resources for the future, especially on children (Ashraf, 2009; Bobonis, 2009; Lundberg, Pollak, \& Wales, 1997; Rangel, 2006; Rubalcava, Teruel, \& Thomas, 2009; Thomas, 1990). Similarly, Duflo (2003) showed that South African children whose grandmothers were entitled to pension funds tended to be taller. This study includes control variables of household composition and the marital status of the household head because empirical evidence suggests that family structure could affect children's health. (Ziol - Guest \& Dunifon, 2014) indicate that children who live with a single father were less likely to have poor health whereas those who lived with a grandparent or those in foster care experienced poor health outcomes. I control for household size since Shieh et al. (2013) report that Vietnam children living in larger families were more likely to use FHI for hospital treatment. To correct for yearspecific variations, which may bias the estimate of $b_{3}, 1$ include year fixed effects, $\tau_{y}$. Therefore, the preferred specification is:

$$
Y_{i t}=\alpha+\tau_{y}+B_{2} \text { Under }_{i}+\text { B3PostPolicy }_{t} \times \text { Under }_{i}+\mathbf{x}_{i t} \mathbf{b}+\varepsilon_{i t}
$$

To assess the validity of the "common trends" assumption underlying the DD model and to examine the potential dynamic effects of the policy, I modify the specification (4.1.2) by interacting Under $6_{i}$ with the year fixed effects. In the absence of the policy, the outcomes in the treated and control group are assumed to follow the same trend. This implies that the coefficients associated with the interaction between the years before the policy change and Under6; should not be statistically significantly different from zero (Meyer, 1995). The specification has the following form:

$$
Y_{i t}=\alpha+\tau_{y}+b_{2} \text { Under }_{i}+\sum_{y=2002}^{2016} \gamma_{y} \times \tau_{y} \times \text { Under }_{i}+\mathbf{x}_{i t} \mathbf{b}+\varepsilon_{i t}
$$

The causal effect represents the intention-to-treat estimator since I assume that all children under six are covered by the law even if some individuals report having no health insurance. One may be concerned that parents may manipulate a child's age to get the treatment. Using observational data makes it difficult to detect potential manipulation in an observed child's 
age. I assume that there is no self-selection into the free health care programme, otherwise the estimates may be biased ${ }^{8}$.

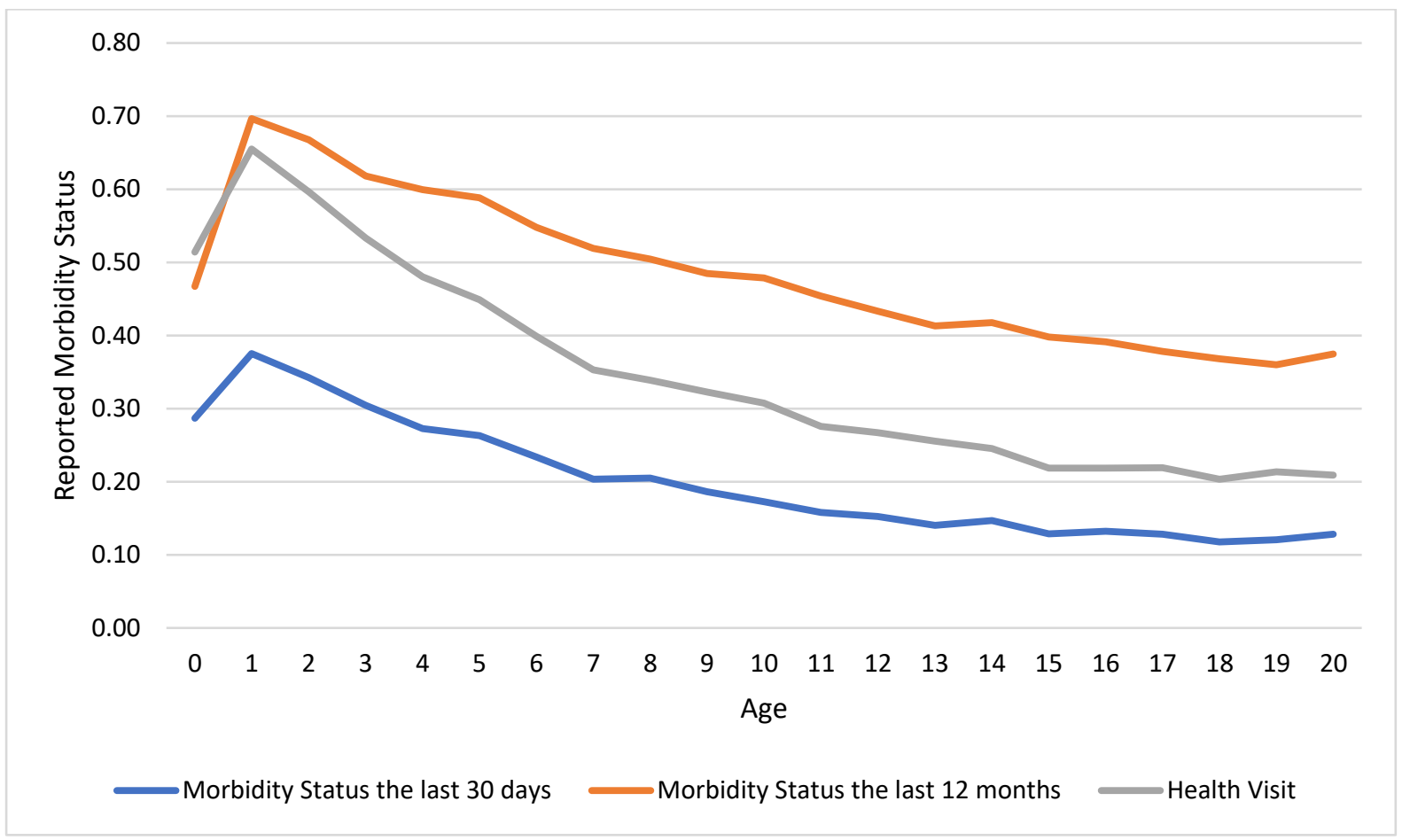

Figure 4. 1 Reported morbidity rates by age (Source: VLSS 93-98 and VHLSS 2004-2008)

4.2 Difference-in-Difference models for the indirect (persistent) effects of the free health insurance policy

Since the policy change affects children across age cohorts, this study uses a DD model to estimate the persistent causal effects of free health care on older children's outcomes. I compare the change in outcomes of children affected by the policy with that of children unaffected after controlling for age and survey year. This comparison relies on the assumption that the outcomes of affected and unaffected children would follow the same trend in the absence of free health care. The following DD OLS regression model estimates the persistent effects of previous exposure to free health care at a younger age:

\footnotetext{
${ }^{8} \mathrm{~A}$ child's age is shown on their birth certificate. To obtain a birth certificate, parents may use the certificate of giving birth released by hospitals, which indicates the time and date the baby was born. Manipulation of age can occur for children whose mothers did not give birth in hospitals. Policy 29/2008/TT-BLETBXH of November 28, 2008 states that children without a birth certificate and certificate of giving birth can request a confirmation paper from a commune officer to obtain free health insurance card.
} 


$$
Y_{i a t}=\gamma_{a}+\lambda_{t}+\beta_{1} D_{i a t}+X_{i a t}^{\prime} \delta+\varepsilon_{i a t}
$$

where: $Y_{i a t}$ is the outcome for a child $i$ aged $a$ observed at survey year $t$. (the outcome variables include health outcomes, school attendance rate and education expenditure); $\gamma_{a}$ indexes dummy variables that indicate age groups ( 6 to 15 years old); $\lambda_{t}$ indexes dummy variables of survey year (2002 to 2016); $D_{\text {iat }}$ is a dummy variable equal to 1 if a child is exposed to free health care and 0 otherwise; coefficient $\beta_{1}$ is the estimated causal effects of previous exposure to free health care at a young age; $X_{i a t}^{\prime}$ is a vector of control variables for individual and household characteristics; and $\varepsilon_{\text {iat }}$ is the error term.

I include a variable indicating the number of years of previous exposure to free health care to specification (4.2.1) to examine the effects of an additional year of free health care on free health care status. Because the policy change in 2005 creates exogenous variation in years of exposure to free health care, I infer an intensity of treatment variable, $I_{i a t}$, that takes a value from 0 to 6 years of free health care. Therefore, $\beta_{2}$ captures the effects of an additional year of free health care on reported health insurance rates. Since one can argue that the effect of changing from 0 to 1 can be different from changing from 5 to 6 years of free health care, to increase the flexibility of the model, I include an intensity of treatment variable $I_{\text {iat }}$ and a dummy variable for free health care status $D_{\text {iat }}$ into (2):

$$
Y_{i a t}=\gamma_{a}+\lambda_{t}+\beta_{1} D_{i a t}+\beta_{2} I_{i a t}+X_{i a t}^{\prime} \delta+\varepsilon_{i a t}
$$

One concern is that the policy started in 2003 that encouraged people to buy voluntary health insurance for the whole family may confound our results. One can also argue that exposure to both policies may affect future health insurance rates. Even though recent studies report that the voluntary health insurance for the family has not been successful in terms of the number of participants (Giang, 2008; Van Tien et al., 2011), I test the robustness of equation (4.2.2) by including a variable, $I^{\prime}{ }_{i a t}$, indicating treatment by the second policy:

$$
Y_{i a t}=\gamma_{a}+\lambda_{t}+\beta_{1} D_{\text {iat }}+\beta_{2} I_{i a t}+\beta_{3} I^{\prime}{ }_{i a t}+X_{i a t}^{\prime} \delta+\varepsilon_{i a t}
$$

Since the free health care policy change in 2005 creates variation in the free health care status of children aged 6 to 15 across age cohorts, there is a concern that children within age cohorts 
can be related to each other. Therefore, I provide robust standard errors of the DD OLS estimators to correct for correlation among these children.

\subsubsection{Difference-in-Difference models for the spill over effects of the free health insurance policy}

This study relies on the implementation of the FHI policy for young children in Vietnam. Since 2005, the country has offered FHI for children under age six. My goal is to leverage this plausibly exogenous variation in the insurance rate for young children over time to retrieve the causal effects on parents' tendency to buy health insurance for older children. Conceptually, my approach compares the insurance rates of older children (age six to 15) with younger siblings before and after the free healthcare was extended to young children (the "first difference"). Of course, a variety of factors may move insurance rates up or down over time, such as higher incomes, lower insurance costs, or rising access to hospitals. To account for these potential time-trending confounders, I estimate the counterfactual trend in insurance rates for these older children with siblings using older children without younger siblings (the "second difference"). I will be successful in retrieving the causal effects of the policy if the trends in insurance rates for these two similarly-aged groups have evolved in parallel in the absence of the policy change. I show that the estimated effect is unlikely to be derived from an endogenous fertility response by parents to the reduced cost of having young children.

I use the DD method to estimate the spill over effects of the policy. At the individual level, I use the DD application on the sample of children aged six to 15 . This sample includes a treated group of children (with eligible younger siblings) and a comparison group of children (without eligible younger siblings). The DD design allows me to compare the changes in health insurance rates between the treated and comparison groups of older children before and after the policy change. I attribute this double difference as the spill over effect of providing free health insurance for young children on older children's health insurance rates.

My DD estimate relies on the assumption that the outcomes of children with younger siblings and those without younger siblings would follow similar trends in the absence of the policy. I 
use the following DD specification to estimate the spill over effect of providing free health insurance for young children on older children's outcomes:

$$
I_{i t}=\propto+\gamma U_{i}+\delta P_{t}+\beta\left(U_{i} \cdot P_{t}\right)+\pi_{t}+X_{i t}^{\prime} \tau+\varepsilon_{i t}
$$

Where: $i$ is a child and $t$ is a survey year. The outcome, $I_{i t}$, includes health outcomes, school attendance rate and education expenditure. The treatment, $U_{i}$, is a dummy variable indicating the presence of younger siblings in the household. This variable can capture differences in health insurance rates between children with and without younger siblings. The post-period, $P_{t}$, is a dummy variable capturing the time trend in health insurance rates across children. The interaction term, $\left(U_{s} . P_{t}\right)$, measures the effect of living with an eligible younger sibling after the policy changed on older children's health insurance rates ${ }^{9} . I$ also include year fixed effects, $\pi_{t}$, to control for common shocks across children over time.

I use a set of variables, $X$, to control for individual and household characteristics ${ }^{10} . I$ include the household head's age ${ }^{11}$ variable to control for potential differences in parents' age between children in the treated group over time. This parents' age gap can affect children's health insurance rates if older parents are more likely to purchase health insurance (i.e., more risk-averse). Therefore, this variable can isolate the potential effects of unobserved factors related to parents' age on these children. Similarly, I control for household head's gender, education level, household size, income, and the presence of other older siblings in the household.

To estimate the spill over effects of the policy change on household outcomes, I use a similar DD specification:

$$
H_{j s t}=\propto_{j}+\gamma U_{s}+\delta P_{t}+\beta\left(U_{s} \cdot P_{t}\right)+\pi T_{t}+X_{j t}^{\prime} \tau+\varepsilon_{j s t}
$$

Where $j$ indicates a household (the observation level). The outcome variables include household wage, income, health insurance coverage, expenditure, and calorie consumption.

\footnotetext{
${ }^{9} \mathrm{I}$ also use interaction terms between year fixed effects $T_{t}$ and sibling effect $U_{s}$ to measure the effects of the policy at certain years in alternative specifications.

${ }^{10}$ I do not include children's age fixed effects in the specification for households.

${ }^{11}$ Since VHLSS does not allow me to link parents to children in extended households, I substitute this with household head's information.
} 
I also use the same set of control variables, $X_{j t}^{\prime}$, to control for household characteristics (including those of the household head). Since I use repeated cross-sectional samples, there can be correlations in individual and household outcomes across survey years. As a result, the sample standard errors can be incorrectly estimated. I alleviate this problem using the robust standard error option provided by the STATA package. 


\section{Chapter 5 THE IMPACTS OF FREE HEALTH INSURANCE POLICY FOR YOUNG CHILDREN}

This study examines the causal effects of providing FHI for Vietnam children under six using DD models and household microdata from the VHLSSs. This chapter reports the DD estimates on the different effects of the policy change on individual and household outcomes. I first present evidence on the direct, persistent, and spill over effects on individual health outcomes. I then report the spill over effects of the policy on the individual educational outcomes and a range of household indicators.

\subsection{The direct health effects}

\subsubsection{Health Insurance Coverage}

In this section, I present the estimated impact of the FHI policy for children under six on the beneficiaries' health insurance status. Since respondents in VHLSS 2004 to 2016 were asked if they had enrolled in any health insurance plan in the past 12 months, the information allows me to create a dummy variable indicating whether the individual was insured. In the next part, I graphically report the impact of the policy change on the reported health insurance rates of children aged zero to five.

Figure 5.1 indicates that the policy had successfully improved the health insurance status of young children. Before the policy change in 2005 , the coverage rate of children under six was 26 percent, which was 36 percentage points lower than the rate of older children (aged six to ten). This difference in insurance rate reflects the fact that school-aged children in Vietnam had more options to buy health insurance than their younger peers. Under the voluntary health insurance scheme for students 


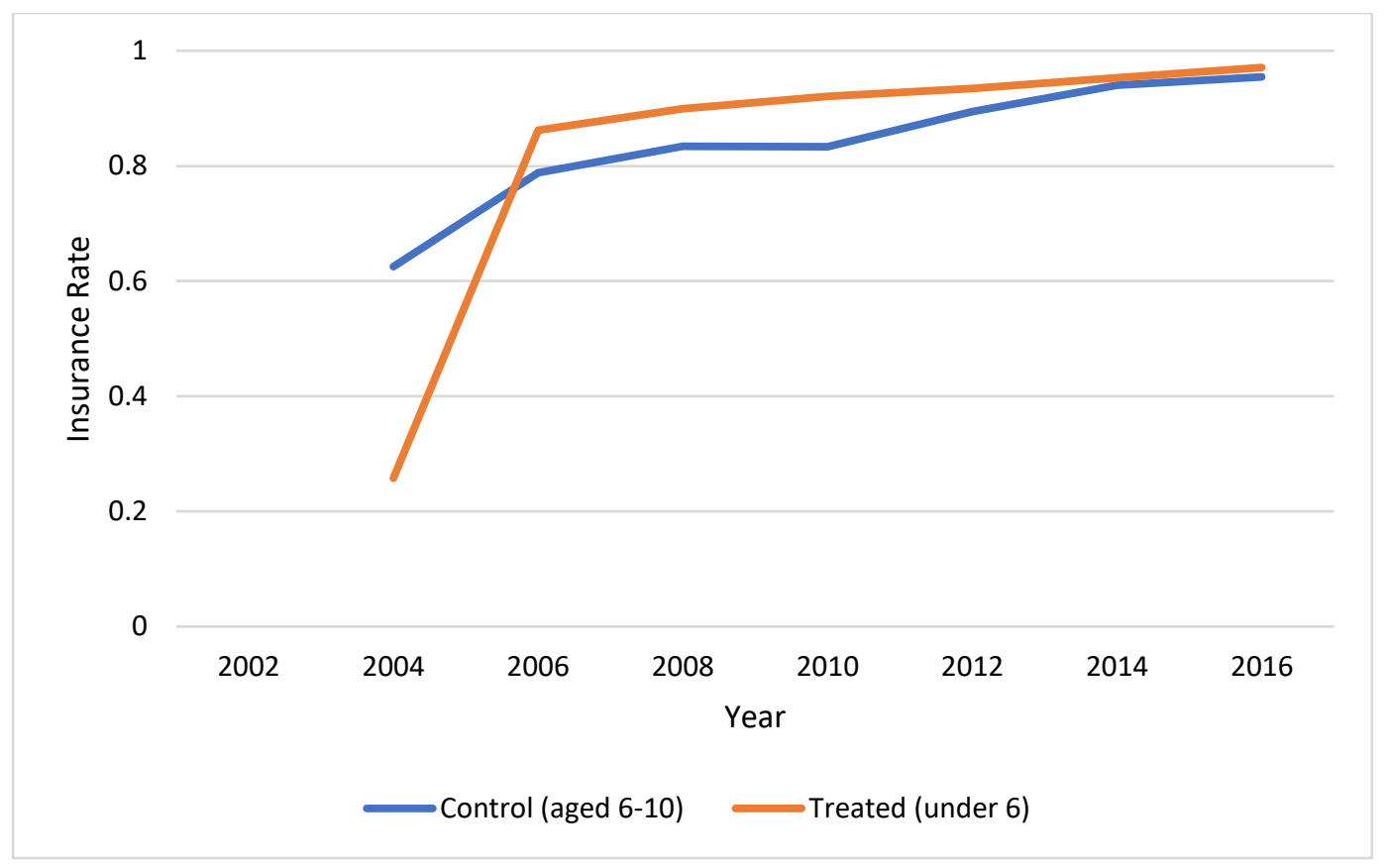

Figure 5. 1 Reported Health Insurance Status of Child Groups

starting in 1994, schoolchildren could enrol in this government-subsidized plan that is more affordable than private health insurance and had less social stigma than the health insurance plan for poor households. Previously, children under six could obtain health insurance only through a private plan or the pro-poor policy. After the FHI policy for young children enacted in 2005, Figure 5.1 shows that the health insurance rate of young children increased by 60 percentage points in 2006 and surpassed the rate for school children in the following years.

Our DD estimates report a positive impact of the policy on the coverage rate of young children. Across specifications, Table 5.1 consistently shows that providing FHI increases the insurance rate of the beneficiaries by 40 percentage points (statistically significantly at $1 \%$ ). Since the policy might have different effects over time and across places, I present evidence on the heterogeneity of its impact. In Table 5.1, Column 2, the estimates of the interaction terms of the treatment status and survey year indicators exhibit a decreasing pattern for the effect of the FHI policy over time. This trend should be interpreted as a success of this policy because it almost approached full coverage for young children and the insurance rate of the control group gradually increased over time.

The results also show evidence on the differential impact of this policy across locations. Table 5.1, Column 3 shows that children in rural areas may reap more benefits from the policy 
change than those in urban areas. Compared with urban residents, the impact of FHI on rural young children is greater by four percentage points (statistically significant at $1 \%$ ). Despite of the disadvantaged conditions of rural infrastructure and a lack of information sources that might prevent households from obtaining healthcare, this finding shows that the policy change can provide better access to health facilities, increase healthcare use and ultimately improve the health status of rural children.

Table 5.1, Column 4, indicates that the FHI policy has differential effects on health insurance rates of children across the six regions of Vietnam. For this specification, I choose the Central Highland (region 4) as the baseline. The results show that the policy has less impact on the insurance rates of children in the Northern Midland and Mountainous Area (region 2) and the Central Highlands. It should be noted that in these two regions live the majority of ethnic groups that have difficult living conditions and low income per capita in the country. In contrast, the policy has greater impact on young children in the Red River Delta (Region 1) and the Mekong Delta area (Region 6) by 2.5 and 3.2 percentage points, respectively. These gaps may be driven by the higher living standards in these regions where residents in the main cities, such as Hanoi and Cantho, have better access to healthcare facilities and information. In the Mekong Delta, even though the fertile land and a complex system of rivers makes life easier, the majority of the rural parts, especially among the Khmer ethnic group, still have a lower education level. This might result in a lack of ability to access useful health information among this ethnic group. Thus, the policy impact is even greater if it disproportionately for these disadvantaged children.

Interestingly, the policy tended to have less impact on the southern part of the country. Compared with the Central Highlands, the policy has less impact on young children in the south east (Region 5) by 2.3 percentage points (the effect is statistically insignificantly at $10 \%$ ). It should be noted that this region with Hochiminh City generates the most revenue and hosts most migrant workers. Since these workers might face difficulties enrolling their young children in the $\mathrm{FHI}$ programme, this can decrease the insurance rate of this region.

I check the robustness of the results by restricting the sample to include children with close age gaps. First, since children across age groups can have different demands for healthcare, I exclude infants and one-year-old children who are arguably more vulnerable to illness. The 
results in Table 5.2 confirm that this exclusion has almost no impact on the DD estimate previously reported. Second, I apply a more conservative restriction to include children aged four to seven. I argue that this approach can yield comparable results but has more flexibility than the Regression Discontinuity model that was used in previous studies. Table 5.3 shows that estimates from this sub-sample have similar signs but lesser magnitude. Specifically, the DD estimates across specifications consistently show that the policy change statistically significantly increased coverage of children aged four to five by 30 percentage points (40 percentage points in the full sample). However, the net effects are unchanged since the postpolicy insurance rates of children aged four and five are consistently higher by 10 percentage points than children aged six and seven.

\subsubsection{Healthcare Utilization}

Previous results show that the FHI policy for young children significantly improves health insurance coverage for the beneficiaries (children under six). This section presents the evidence on whether this improvement can be translated into higher healthcare utilization among these young children. The measures of utilization include the probability of visiting health facilities and the number of outpatient and inpatient visits in the past 12 months. In the following parts, I report the DD estimates of the impact of this policy change on these outcomes.

\subsubsection{Health visits}

The variable distribution of health visits shows that 40 percent of the children in the full sample (aged zero to ten) had at least one health visit. As can be seen in Figure 5.2, children under six are generally more likely to visit health facilities than older ones. Although the FHI policy change in 2005 immediately improved insurance coverage for young children, the health visit rates of children in the treated and control group shown in Figure 5.2 tended to follow the same trend until 2010. After that, the probability of health visits by young children continued to increase and the rate for older children went down. This implies that the policy might take longer to have significant effects on healthcare utilization. 


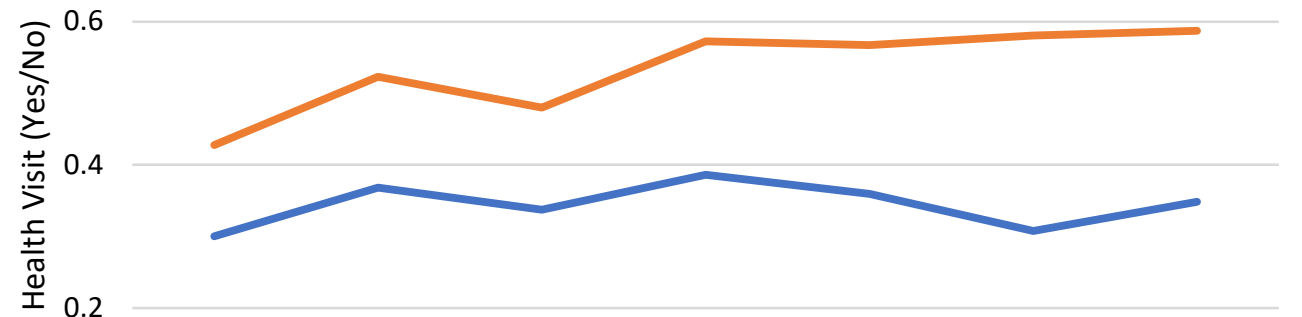

0

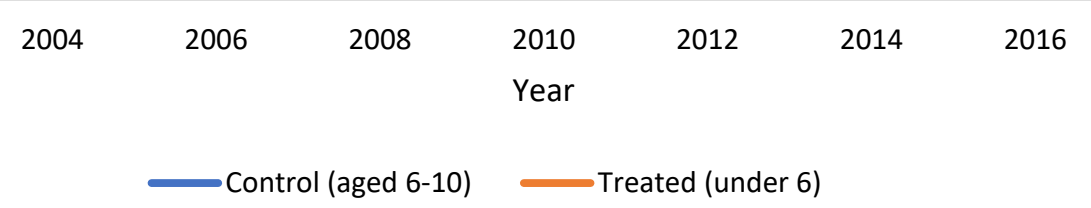

Figure 5. 2 Children's Health Visits in the Last 12 Months

Table 5.4 shows evidence of the positive impact of the policy change on the likelihood of visiting health facilities. After controlling for the characteristics of the household and its head, the DD estimate in Column 1 indicates that the FHI policy increases the chance of health visits by eight percentage points (statistically significant at $1 \%$ ). This means that, because of the policy, children under six are more likely to have more health visits than their older peers.

I also present evidence on the heterogeneity of the impacts of the policy across locations and over time. The coefficients of the interaction terms in Table 5.4, Column 2, indicate that the impact is more pronounced over time. Indeed, the change in the effect from 0.029 to 0.13 between 2006 and 2016 suggests that the policy had a larger effect on health visits after 10 years of implementation than it did in the early phase. I also find that the policy might have less impact on the likelihood of children in the rural areas visiting health facilities by five percentage points compared with their urban peers.

The results in Table 5.4, Column 4, indicate that the policy has had a uniform effect across regions. Although the policy had more impact on young children in the Central Highlands than those in the other areas (except the Red River Delta) by one to three percentage points, these estimates are statistically insignificant at the $10 \%$ level. Since the Central Highlands and the Northern Midland and Mountainous Area are two disadvantaged parts of the country with a 
majority of indigenous groups, one would expect that the policy could have more impact on healthcare use in there. However, the findings do not support coverage expansion after the policy change translating into higher healthcare demand in these regions than elsewhere.

It is noteworthy that there are other policies and programmes that target young, vulnerable children in Vietnam. To the best of my knowledge, the law on protecting children took place a year before the FHI policy was implemented in 2005. This law can facilitate other policy interventions such as increasing investment in healthcare infrastructure or the education system that potentially improve the healthcare utilization of those living in difficult conditions.

These findings are robust to the restriction of the data to include children with similar physical conditions. After excluding infants and one-year-old children, Table 5.5 shows that the estimated effects are reduced in size but not sign. However, as I include only children whose ages are near the cut-off point, the results in Table 5.6 become insignificant. Therefore, I argue that the policy might have most impact on young children so their absence in this sample reduces the size of the effect.

\subsubsection{Outpatient visits}

Though outpatient care, such as seeking care at private clinics run by doctors or purchasing medicine over the counter without a prescription, is common in Vietnam, these services are not covered by insurance plans. So, if FHI policy for young children can motivate parents to visit public health facilities where expenses are covered, I would expect a decreasing trend in outpatient use. However, Figure 5.3 illustrates that the number of outpatient visits by children in the sample increased over time, and under-six children tend to use more outpatient care than older ones. Three years after the policy change, the utilization of the two groups of children follows the same trend. This implies that the FHI policy might have no immediate effect on outpatient use among targeted children. Indeed, the gap in the outpatient visits between the two groups seems to be slightly wider since 2010 .

The regression results show a positive impact of the $\mathrm{FHI}$ policy on young children in outpatient visits. The OLS estimates in Table 5.7 indicate that the FHI policy can increase the outpatient use of children under six by 0.29 visits (statistically significant at 1\%), which is 22 percent of 
the average number of outpatient visits. Since the number of children reporting no visits accounts for 40 percent of the distribution of outpatient use (see Figure 5.4), I perform a Poisson regression to test the stability of the OLS estimates. Though the goodness-of-fit test indicates that the Poisson model might not fit the data well, there is still a positive impact of the policy on outpatient visits.

The results also show that there are variations in the effects of the FHI policy across regions and over time. In Table 5.7, Column 2, the coefficients of the interaction terms suggest a lag in the policy effect since it started to have larger, significant impact five years after the implementation. The results in Table 5.7, Column 3 indicate that the policy has less impact on young children in rural areas by 0.4 outpatient visits, compared to their urban peers. This gap might result from the lack of healthcare infrastructure in rural parts of the country.

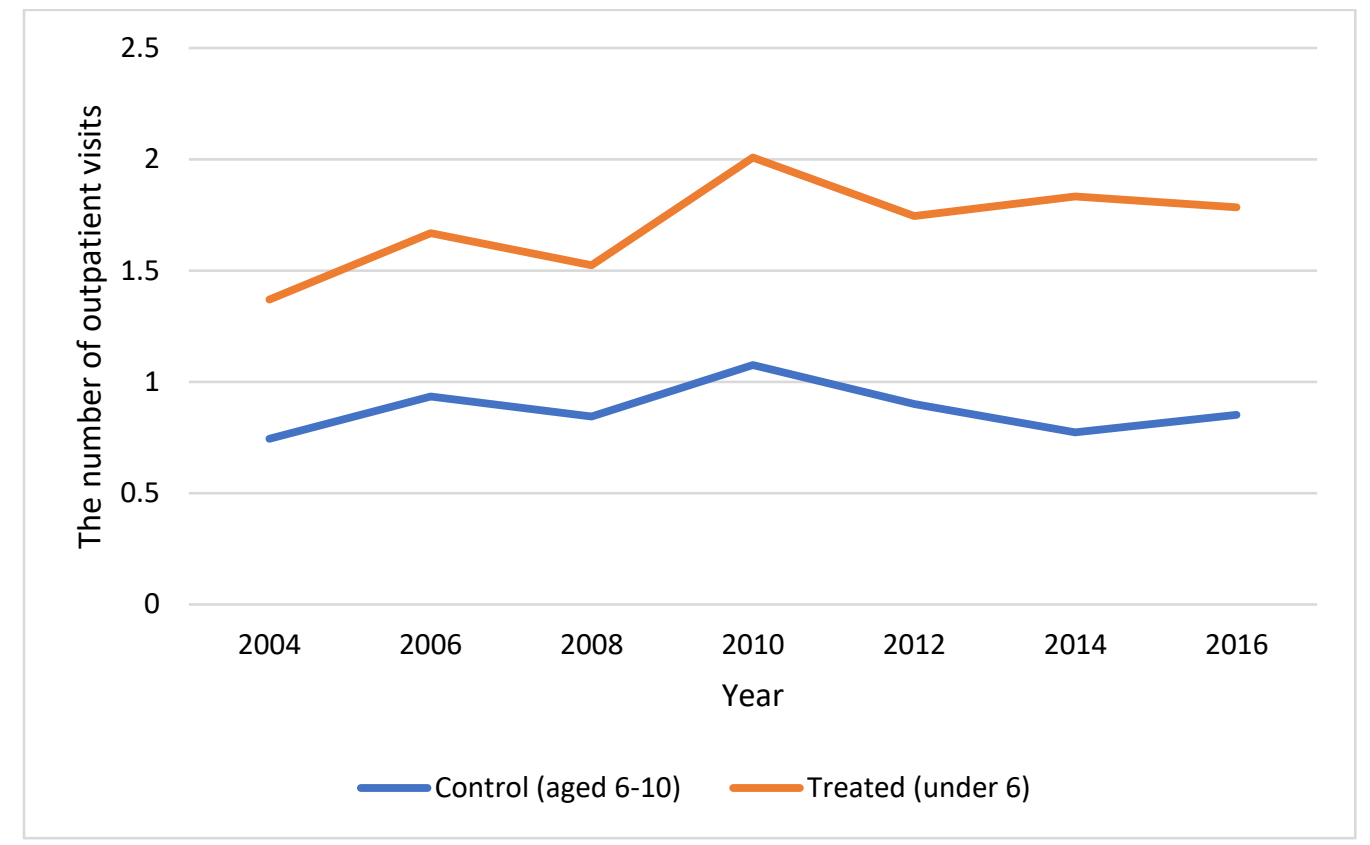

Figure 5. 3 Children's Outpatient Visits over the Last 12 Months

I find that the policy effects on outpatient visits are significantly greater in southern regions, especially in the Mekong Delta. The findings in Table 5.7, Column 4 further show that the policy has less utilization impact in the Northern Midlands and Mountainous areas by 0.3 visits. The gap can be because the outpatient services in southern Vietnam are more available and easily accessed compared with those in the northern mountainous areas. 


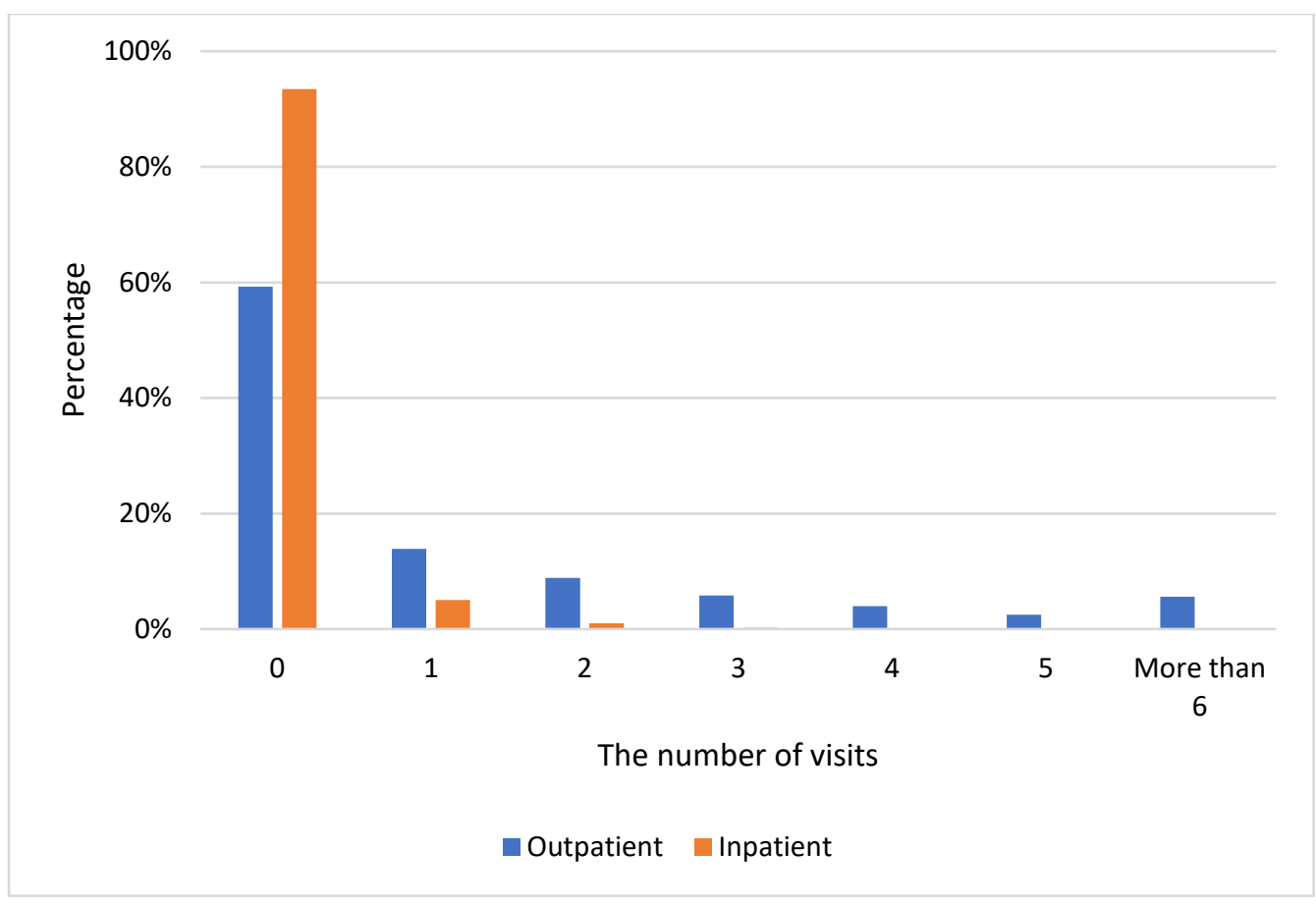

Figure 5. 4 The Distribution of Children's Healthcare Utilization

Table 5.8 shows that the results remain stable with the exclusion of infants and one-year-old children from the data sample. As I narrow the age difference among children, the estimates in Table 5.9 decrease substantially and become insignificant. As earlier with health visit outcome, the findings are consistent with the notion that the policy exerts more impact on young children than older peers (i.e., infants are the most vulnerable to diseases so need more healthcare).

\subsubsection{Inpatient visits}

Under the FHI policy for young children, inpatient expenditure is fully covered. However, because hospitals in Vietnam are overcrowded and treatment is costly (for the uninsured and healthcare system), inpatient admission is often the last resort in seeking healthcare in Vietnam. If the FHI policy can eliminate the financial burden of hospitalization as intended, I expect to see an increase in inpatient use among young children. As can be seen in Figure 5.5, there is an increasing trend in inpatient use among young children over time, while utilization among older children shows no major change.

I find limited evidence on the positive effect of the FHI policy on inpatient utilization among young children. In Table 5.10, the OLS estimates show that the FHI policy has a slightly greater 
impact on children under six by 0.02 visits (equal to 22 percent of the sample mean of inpatient visits). It should be noted that about 93 percent of children in the sample reported no inpatient visits in the past 12 months. Given that the inpatient admission is rare, I perform a Poisson regression for a robustness check.

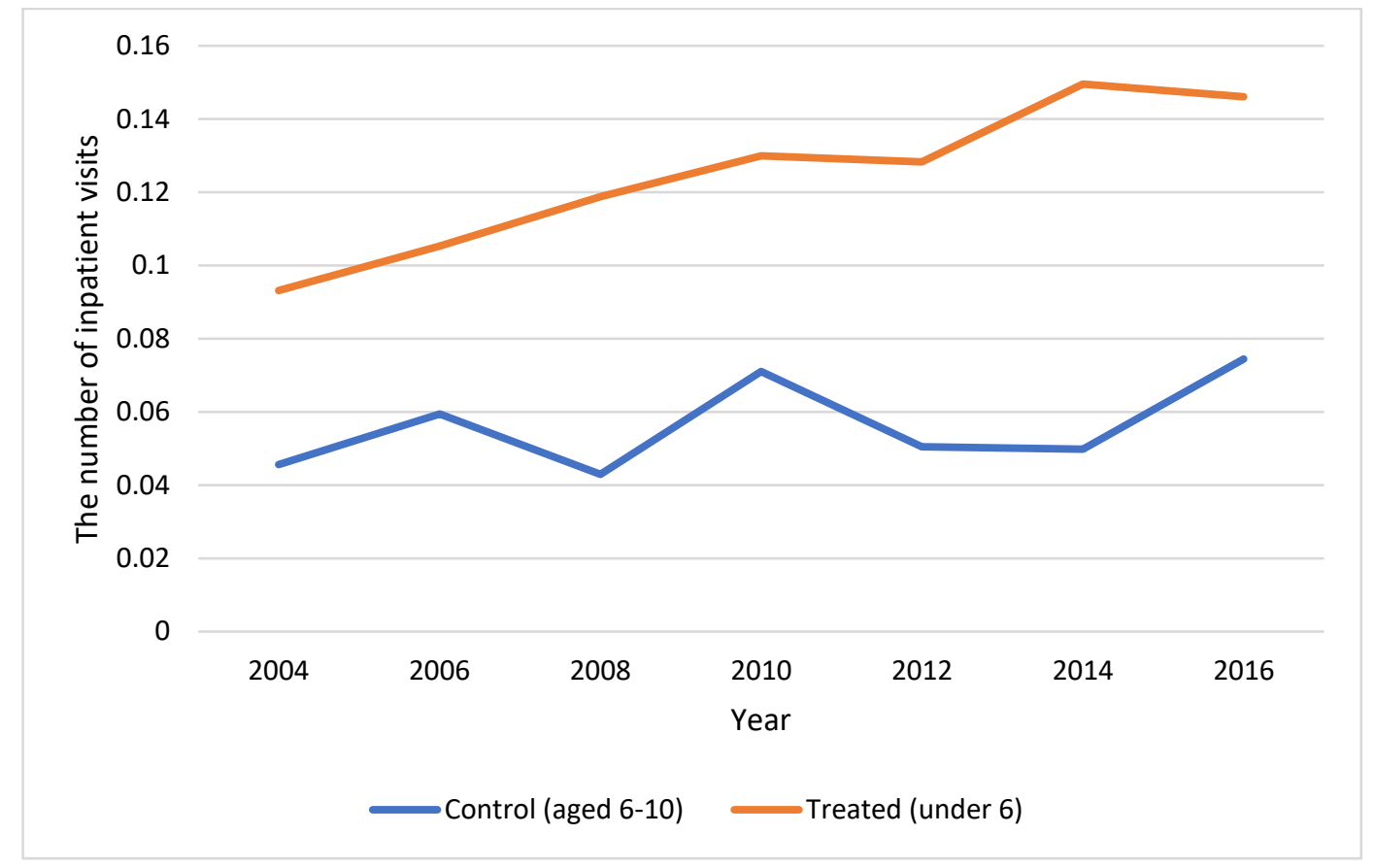

Figure 5. 5 Inpatient Admissions for the Last 12 Months

The results from the Poisson models cannot reject the null hypothesis that the policy has any impact on inpatient use. I also checked the robustness of the results by restricting the sample to include children who are less vulnerable to illness and are of a similar age. The results in Tables 5.11 and 5.12 show no significant effects of $\mathrm{FHI}$ on the inpatient care of children aged two old and older. Therefore, it might be that the policy is beneficial only to very young children. The lack of impact on inpatient care can also be because of the competing effects that local health care can reduce the occurrence of severe conditions and hence decrease the inpatient use by insured individuals.

The OLS models provide some evidence of heterogeneity in the effects of the policy change on the inpatient use across regions and over time. The results in Table 5.10, Column 2, suggest that the FHI policy might take some time to have a significant impact on the use of inpatient services. In contrast to the effect on outpatient use, the estimate in Table 5.10, Column 3, shows that the policy has no differential effects on rural and urban children (the effect is small 
and statistically insignificant at conventional levels). Table 5.10, Column 4 , shows that the policy has less effect on children in southern Vietnam than those in the mountainous areas (Northern Midlands and Mountainous areas and the Central Highlands). Even though these estimates are statistically insignificant, they may give a glimpse of how children seek healthcare across regions. Though drugstores and private clinics run by doctors are popular in Vietnam, the availability of these facilities can affect the choice between outpatient and inpatient care. Suppose that children in the south can easily access these types of healthcare and that outpatient care is perceived by patients as more convenient, this can explain why outpatient and not inpatient services are more common in the south than in other regions. Another explanation for the greater impact on the inpatient use in the Central Highlands, Northern Midlands and Mountainous areas is that children in these disadvantaged regions might have worse health conditions that need be treated in hospitals. Therefore, the FHI policy that provides these children with free access to any care can increase inpatient use. In contrast, the impact of $\mathrm{FHI}$ on inpatient use is more pronounced among children in the north of the country, especially in the Red River Delta. This finding implies that inpatient care is preferable in the north whereas outpatient care is more popular in the south.

\subsubsection{Healthcare Expenditure}

Previous sections show that the FHI policy has a positive effect on the probability of visiting health facilities as well as on the number of outpatient and inpatient visits. Previous studies found that the insured tended to use more healthcare, thereby increasing healthcare expenditure. Though FHI for young children in Vietnam aims to provide greater access to healthcare, its primary goal is to protect families from financial hardship because of illness. In this section, I present evidence on the policy's ability to reduce out-of-pocket health payments for young children. The following parts report the DD estimates of the impact of the FHI policy on individual, outpatient, and inpatient health expenditure.

\subsubsection{Individual health expenditure}

Although the FHI policy aims to reduce out-of-pocket health expenses, Figure 5.6 shows that it might only contain the healthcare cost and only for a year in 2006 because, after that, the expense has risen over time. Though young children tend to incur higher health spending than 
the older children, the gaps in health expenses seems to have expanded since 2012. It should be noted that older children can enrol in an insurance plan for school children. Thus, the increases in health expenditure on children in the sample may signal that the health insurance programmes might be ineffective at containing rising healthcare costs.

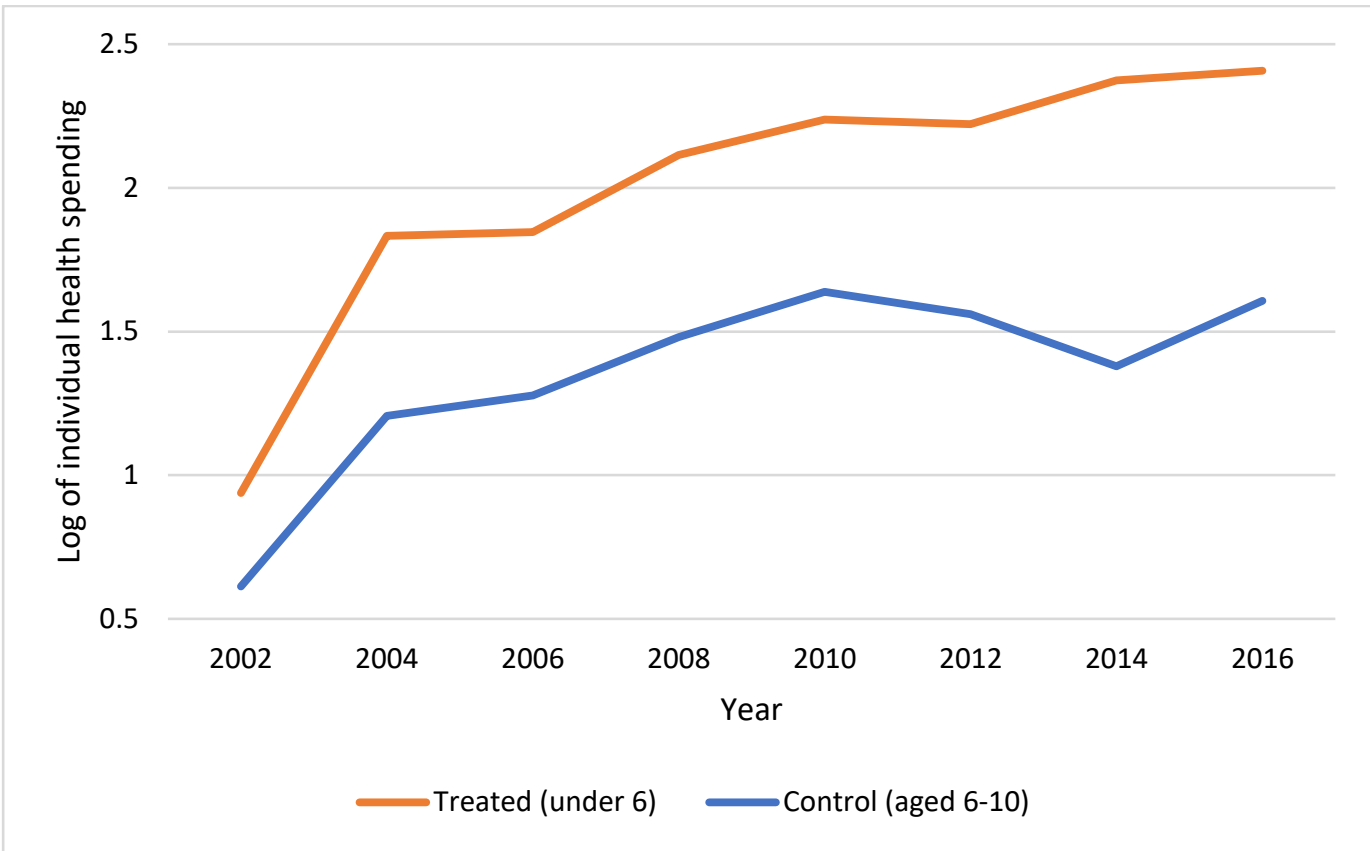

Figure 5. 6 Out-of-Pocket Health Expenditure on Different Aged Children

I find that the provision of health insurance has a positive impact on healthcare spending on young children. The OLS estimates in Table 5.13, Column 1, show that the policy change increased spending by 34 percent (statistically significantly at 1\%). Since health expenditure is a truncated variable, I perform Tobit regression to detect any misspecification problem using OLS models. The Tobit estimate confirms that the policy has a sizeable, positive effect on personal health spending.

The results indicate that the policy has a differential impact on health expenses across regions and over time. In Table 5.13, Column 2, though spending is generally higher since the policy was implemented, the policy impact on health expenditure has been greater since 2012, seven years after implementation. There is a stark difference in the size effect of 35 percentage points between 2010 and 2014. This is concerning because it signals that the policy might be ineffective in providing financial protection for the insured. 
In Table 5.13, Column 3, I find that the impact of the policy change on health payments is more pronounced for urban children than for those in rural areas. As pointed out in previous sections, rural children might incur fewer health expenses because of lower health insurance rates and healthcare use. Therefore, there is less impact on health spending among rural children that should not be interpreted as the financial protective effect of the policy. The estimates in Table 5.13, Column 4, suggest that the impact of $\mathrm{FHI}$ on health expenditure in the southern part of the country is significantly greater than in other regions. Specifically, the effects on spending are greater on children under six in the south-east and Mekong Delta than in the Central Highlands by 57 and 37 percentage points, respectively. As previously indicated, the outpatient care is more prevalent in the south. If this type of service involves seeking care from private clinics or drugstores, the insured cannot claim reimbursement. Therefore, I argue that the higher level of out-of-pocket payments in the south is because of outpatient service, which is not covered by the FHI policy.

I test the sensitivity of the results by focusing on children of similar physical condition and age. Using a sample of children aged four to seven, Table 5.15 shows a significant drop in the size effect of the policy change on the health expense of these children, but the sign remains unchanged. These results imply that the policy increases health expenditure across age groups even though older children have less demand for healthcare.

\subsubsection{Outpatient and inpatient expenditure}

I find that the FHI policy has a positive effect on outpatient and inpatient spending. In Table 5.16, Column 1, the OLS DD estimate is that the policy increases the outpatient expense of children under six by 33 percent (statistically significantly at $1 \%$ ). This is consistent with previous evidence on the positive impact of insurance on total individual health expenditure. Table 5.19 reports a statistically significant increase in inpatient cost among young children by six percent after the policy change. As previously argued, the costs of inpatient care can typically be reimbursed under the FHI policy. This result shows that even if the insured children use only the covered inpatient care, they still incur higher expenses. Although the government has implemented a range of policies to regulate the price of healthcare services and drugs, it is unable to control related costs such as transport or under-the-table money that can drive up health expenses significantly. 
My results show heterogeneity in the $\mathrm{FHI}$ effects on the outpatient spending on young children. In Table 5.16, Column 2, the policy has had a larger impact on outpatient expenditure among children under six since 2014 compared with earlier years. Table 5.16, Column 3 , indicates a lesser spending effect on rural children than urban children by 54 percentage points. Additionally, the results in Table 5.16, Column 4 show that FHI appears to have a greater impact on outpatient spending among children in the wealthier part of the country (the south and the Red River Delta) than in the poorer regions (Central Highlands and the Northern Midlands and Mountainous Areas).

In contrast, Table 5.19, Column 2, shows no time trend in the effect on inpatient expenditure, but Column 3 shows that the policy has a greater influence on the expenditure on rural children than on urban children (but the estimate is statistically insignificant at $10 \%$ ). The estimates in Table 5.19, Column 4, indicate that the policy's impact is greater on inpatient payments for children in the northern and central Vietnam than for those in the south.

These results on the impact on the cost of outpatient and inpatient services reflect the healthcare use pattern that was reported in the section 5.1.2.2 and 5.1.2.3. Specifically, outpatient care is widely used in the south, but inpatient service is more common in the centre and north. Regardless of the service type, the estimates suggest that the FHI policy cannot contain costs for the insured since those who demand more healthcare have to pay more for it.

Again, I use Tobit regression to test whether the OLS results are sensitive to the choice of specification. The Tobit estimates of the effect of the FHI policy on outpatient and inpatient spending are positive and statistically significant at $1 \%$ I also restrict the sample to exclude very young children who might have higher healthcare demands. With the effects on outpatient spending, the results in Tables 5.17 and 5.18 consistently show that the FHI policy has a positive impact and the effect is more pronounced for young children. Tables 5.20 and 5.21 indicate that the policy change might increase inpatient expenditure among young children, but not the older ones covered by the FHI. 


\subsubsection{Health Status}

One of the two main goals of the FHI policy for young Vietnamese children is to promote the health status of the beneficiaries. Recent studies report the positive impacts of health insurance policies on a range of health outcomes, including mortality rate, acute disease, malnutrition and aggregate health index. As presented in the section 5.1.1 and 5.1.2, the policy change improved health insurance coverage of young children, which can translate into a higher level of healthcare utilization. In this section, I show the evidence of the direct health effects of the policy on the morbidity (as reported in the last 30 days and past 12 months) of children under six.

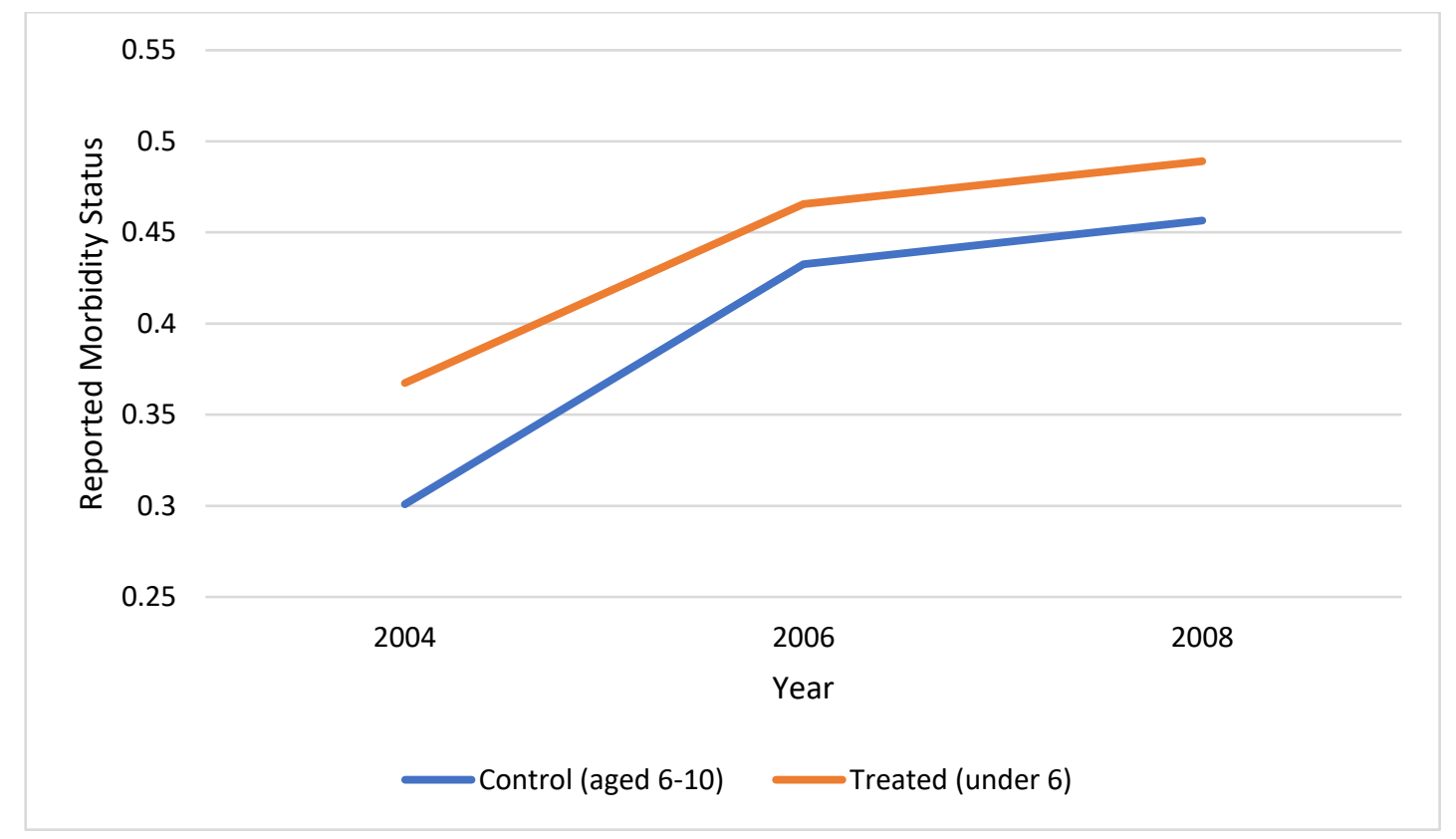

Figure 5. 7 The Last Month Morbidity Rate of Two Age Groups of Children

As illustrated in Figures 5.7 and 5.8, young children generally have higher monthly and yearly morbidity rates than older children by five and ten percentage points, respectively. The descriptive statistics also show that insured children are seven-percentage points more likely to report with a health issue than uninsured ones. Although this association might imply an influence of insurance coverage on self-reported health status, the two figures do not portray any substantial changes in the health status of young children following the policy change. In fact, the regression results show a limited impact of health insurance on the likelihood of reporting illness among young children. In Tables 5.22 and 5.25, Column 1, the policy change 
can reduce the 30 -day-period morbidity rate by 2.8 percentage points (statistically significant at $10 \%$ ) while having no meaningful impact on the health status in the past 12 months.

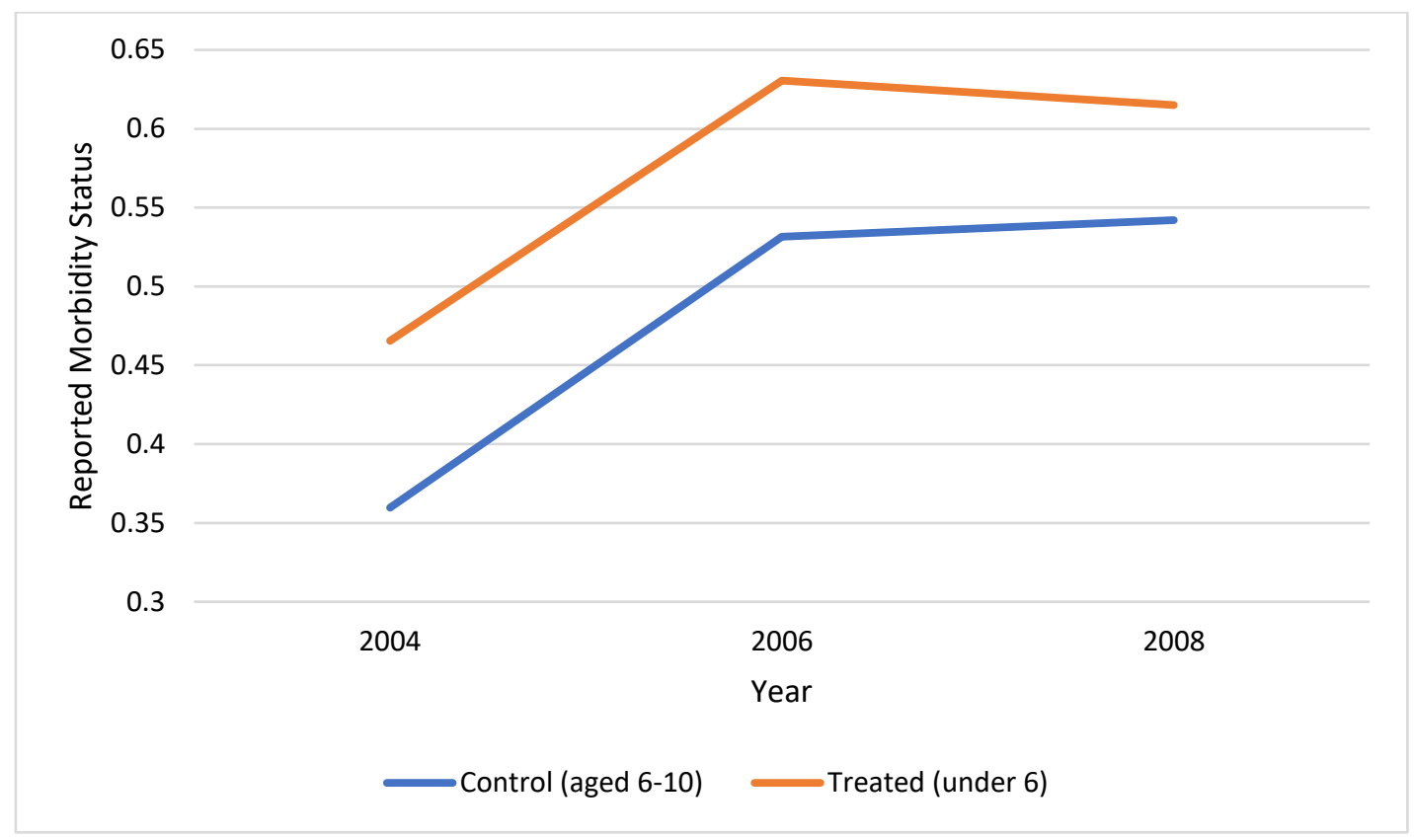

Figure 5. 8 The Last 12 Months Morbidity Rate for Two Groups of Children

The rest of these tables report evidence on the heterogeneous effects of the FHI policy across locations. Table 5.25, Column 4, shows that the policy might have a greater effect on the morbidity rates of children in the Northland Midlands and Mountainous areas than in the Central Highlands. Although the descriptive statistics show that household income is lower in the Northern Midlands and Mountainous areas, the policy impact tends not to increase the wellbeing of these children who live in economically disadvantaged conditions.

It should be noted that the estimates are sensitive to the restriction of the sample to children of specific age groups. In Tables 5.24 and 5.27, as I include those with a narrower age gap (four to seven), the impact doubles and becomes more statistically significant. In contrast, the exclusion of infants and one-year-olds reduces the estimates in Tables 5.23 and 5.26 reduce and they become insignificant. These sensitivity tests suggest that the policy might be more beneficial to older children than younger ones, and that the choice of comparison group matters. 


\subsection{Persistent Health Effects}

In this section, I present evidence on the prolonged impact of the FHI policy on children who were exposed to this policy change at a young age. The policy change created variations in the duration of exposure to $\mathrm{FHI}$ among children of different birth cohorts. For example, in Figure 5.9, within the same age groups, children in the Cohort A could have one year of FHI whereas those in the Cohort B were too old to enrol in the programme. My analysis exploits these variations to estimate the persistent effects on health outcomes using pooled repeated cross-sectional microdata of children aged six to 15 . In the following discussion, I provide evidence on whether being exposed and the intensity of exposure to the FHI policy can affect insurance coverage, healthcare use, expenditure and health status.

\subsubsection{Health insurance coverage}

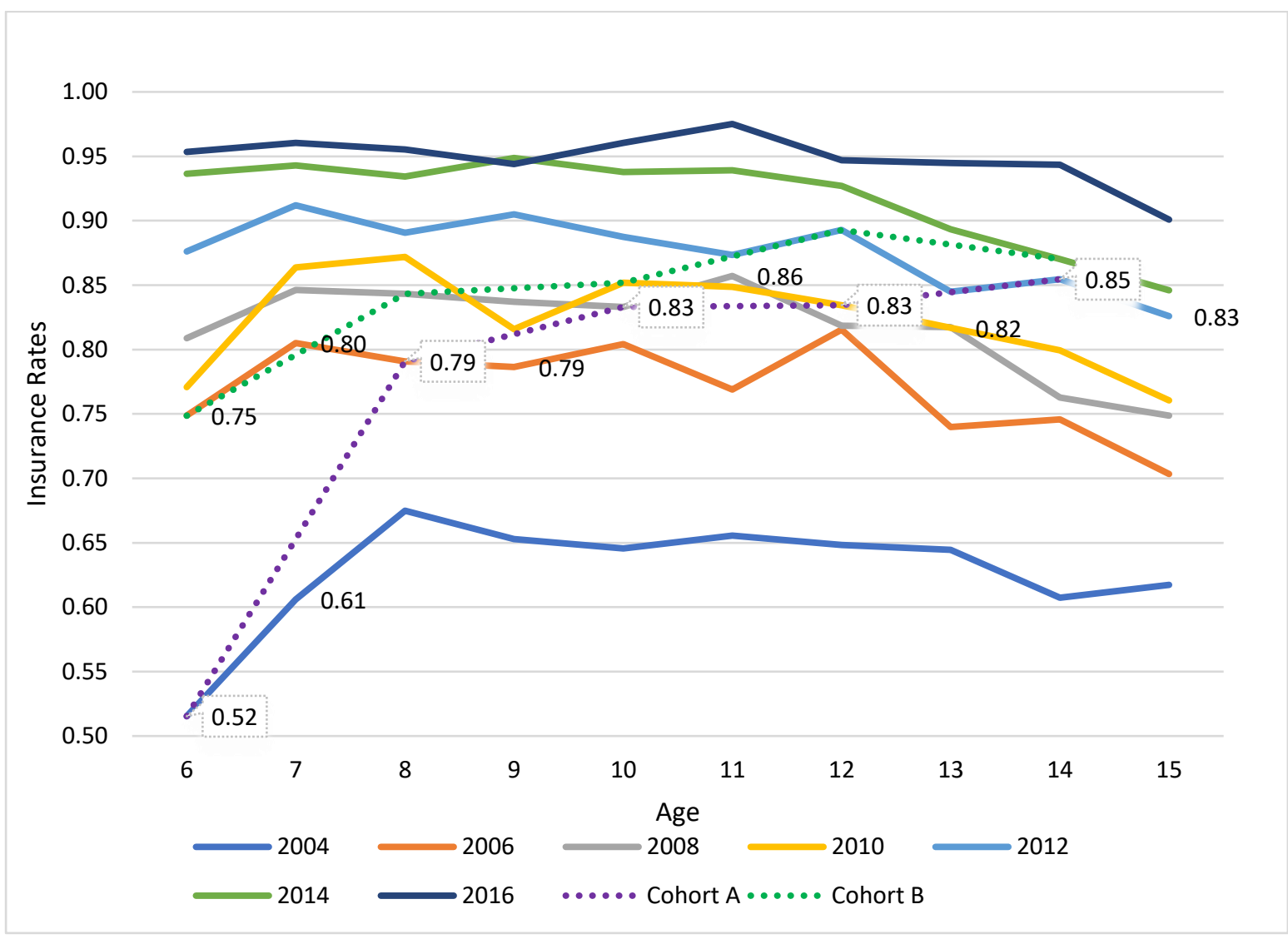

Figure 5. 9 The Persistent Effects on Health Insurance Rates of Children

Figure 5.9 depicts the insurance coverage of children aged six to 15 who were respondents in VHLSS 2004 to 2016. As can be seen, there are three sources of variation in insurance rate: 
across and within age groups as well as between birth cohorts. I will illustrate the policy impact on health insurance status using two groups of six and seven-year-old children. One year after the policy change, the insurance rate of children aged six increased by 23 percentage points but the increase among children aged seven was 19 percentage points. Children aged six in 2006 could experience one year of FHI, the others were too old to enrol in the programme. Assuming that the change in insurance status of children aged seven (who received no treatment) is a valid counterfactual for children aged six in the absence of treatment, I then attribute the difference in coverage between these two groups as the persistent effect of exposing to one year of FHI. The same logic applies to children in other age groups.

I find evidence of a positive impact of the FHI policy on insurance coverage of children who were exposed to this policy at a young age. In Table 5.28, Column 1, conditioning by prior insurance status, an additional year of exposure to $\mathrm{FHI}$ increases future health insurance rate by 0.3 percentage points (statistically significant at $10 \%$ ). Although this finding is consistent with previous studies that report a long-term link between childhood environment and adult outcomes, my results shed some light on the policy impact right after children become ineligible for FHI. Since the Vietnamese government has been encouraging its citizens to participate in the SHI programme, $\mathrm{FHI}$ for young children should be considered a promotion or trial that could increase adult enrolment.

My results further suggest that the policy effects can vary across living location. In Table 5.28, Column 2, relative to urban residents, the estimate indicates that the policy has a larger impact on rural children by 8.2 percentage points (significant at 1\%). In contrast, Table 5.10, Column 3, shows that the persistent effects are more pronounced on children from wealthier regions, including the Red River Delta and southern part of the country. One potential explanation for this gap is that parents from advantaged areas have more and better experience of $\mathrm{FHI}$ on their young children than those in the Central Highlands where the access to healthcare might be limited. Realizing the benefits of earlier FHI, these parents may continue to health insurance for their children. 


\subsubsection{Healthcare utilization}

I find that entitlement to $\mathrm{FHI}$ at a young age can increase healthcare utilization among children aged six to 15. In Table 5.29, Column 1 children who were exposed to at least one year of FHI are more likely to visit healthcare facilities than those without exposure by 2.3 percentage points (statistically significant at 1\%). The size of the effect, which is smaller than the direct effect on health visit rate, might reflect the fact that older children are less vulnerable to illness, thereby having a lower demand for healthcare.

In Table 5.29, Column 3, the estimates indicate that FHI might have a larger impact on the likelihood of visiting health facilities among children in southern regions. As reported in Section 5.1.1, the affected children in these regions are more likely to purchase health insurance that can cover the higher level of utilization. Also, compared with the Central Highlands, the southern part of the country has better economic conditions and healthcare infrastructure that can improve the outreach of the policy and facilitate healthcare utilization.

I also find that the FHI policy has a limited impact on outpatient care and no significant effect on inpatient utilization. Indeed, the results in Table 5.30 show that previous exposure to the policy increases the number of outpatient visits by only 0.09 visit (statistically significant at 1\%). In contrast, Table 5.31 shows a negligible impact of the policy on the number of inpatient uses. Since these estimates are consistent with those from the Poisson regression, I argue that the results are not susceptible to the misspecification issue.

\subsubsection{Healthcare expenditure}

My findings indicate that the FHI policy can have a prolonged impact on healthcare expenditure of children aged six to 15 . Table 5.32 shows that exposure to $\mathrm{FHI}$ at a young age consistently increases the individual health spending by 13 percent. Moreover, an additional year of FHI can further increase the expense by 3.6 percent. These estimates are statistically significant at $1 \%$. It should be noted that my results can be biased because of the misspecification issue, so I perform Tobit regression for healthcare expenditure and the estimates are consistent in sign but statistically insignificant. 
Table 5.33 shows that the policy has a positive impact on spending on outpatient services. Since inpatient care is rare and outpatient costs account for the large proportion of total health spending, the estimates in this table reflect the impact on overall health expenditure. I find that the policy has no significant effect on inpatient expenditure (see Table 5.34). Previous findings report that the FHI policy has a small impact on the number of outpatient visits among children aged six to 15 . Thus, the increase in outpatient spending might signal that healthcare has become more expensive.

I find evidence of heterogeneity of the impact of the policy change on healthcare expenditure. As reported in Tables 5.32 and 5.33, Column 2, compared with urban areas, the effects on total healthcare cost and outpatient expenses are lower in rural areas by 12 and 21 percentage points, respectively. Since the policy has less impact on outpatient use among rural children, the lower outpatient cost might be interpreted as a result of low utilization, not that healthcare expenditure is more affordable in rural areas.

Similarly, the effects on healthcare spending and outpatient costs vary across regions. Compared with the Central Highlands, the policy has less impact on healthcare expenditure in the Northern Midland and Mountainous areas but has substantial effects in southern regions (as reported in Column 3 of Table 5.32 and 5.33). In contrast, Table 5.34, Column 3 shows that the policy has less impact on inpatient spending in Mekong Delta region. This pattern is consistent with the healthcare use trend previously presented. Indeed, since healthcare costs increase as a result of higher utilization, these findings suggest that health insurance in Vietnam might be unable to provide financial protection. Under the hospital autonomy policy, hospitals in Vietnam are encouraged to generate more revenue, which gives them the incentive to perform unnecessary but costly tests. Since these costs are unlikely to be covered by health insurance plans, patients have to bear the burden. Further, the cost of transport, accommodation and under-the-table money for doctors can exacerbate increasing healthcare costs. These issues pose a huge challenge for current health insurance policies in Vietnam. 


\subsubsection{Health status}

Although the policy might increase healthcare utilization among children aged six to 15 , this increase is translated into better health outcomes. As reported in Tables 5.35 and 5.36, the policy has no significant impact on two measures of health status: self-reported morbidity in the last 30 days and past 12 months. This is consistent with the notion that older children, who are not as vulnerable to illness as young children, might have less demand for healthcare.

\subsection{Spill Over Health Effects}

In previous sections, I showed that providing free health insurance for young children can affect their health-related outcomes directly and persistently. This section reports whether the policy has any impact on health outcomes for untargeted children. To estimate the health spill over effects, I use the DD model to compare the changes in the outcomes for the treated group (children aged six to 15 who lived with under-six siblings) with the changes in the outcomes for the control group (children of similar age who do not live with targeted siblings). The following parts presents the DD estimates of the $\mathrm{FHI}$ impacts on health insurance coverage, healthcare utilization and expenditure and morbidity.

\subsubsection{Health insurance coverage}

Figure 5.10 shows that the health insurance rate of children aged six to 15 follows an upward trend even before the provision of $\mathrm{FHI}$ for children under six. The increased coverage may reflect either growing popularity of voluntary health insurance for school children started in 1994 or a positive effect of the Law on Children Protection enacted in 2004. Along with the overall economic development of Vietnam during this period, these pro-children policies through subsidy and information might have a positive impact on the coverage of school-aged children. My interest is to examine whether living with young siblings who were entitled to $\mathrm{FHI}$ can benefit older children. As can be seen from Figure 5.10, the expected coverage rate of treated children surpassed those in the control group one year after the policy change and remained higher in the following years. 


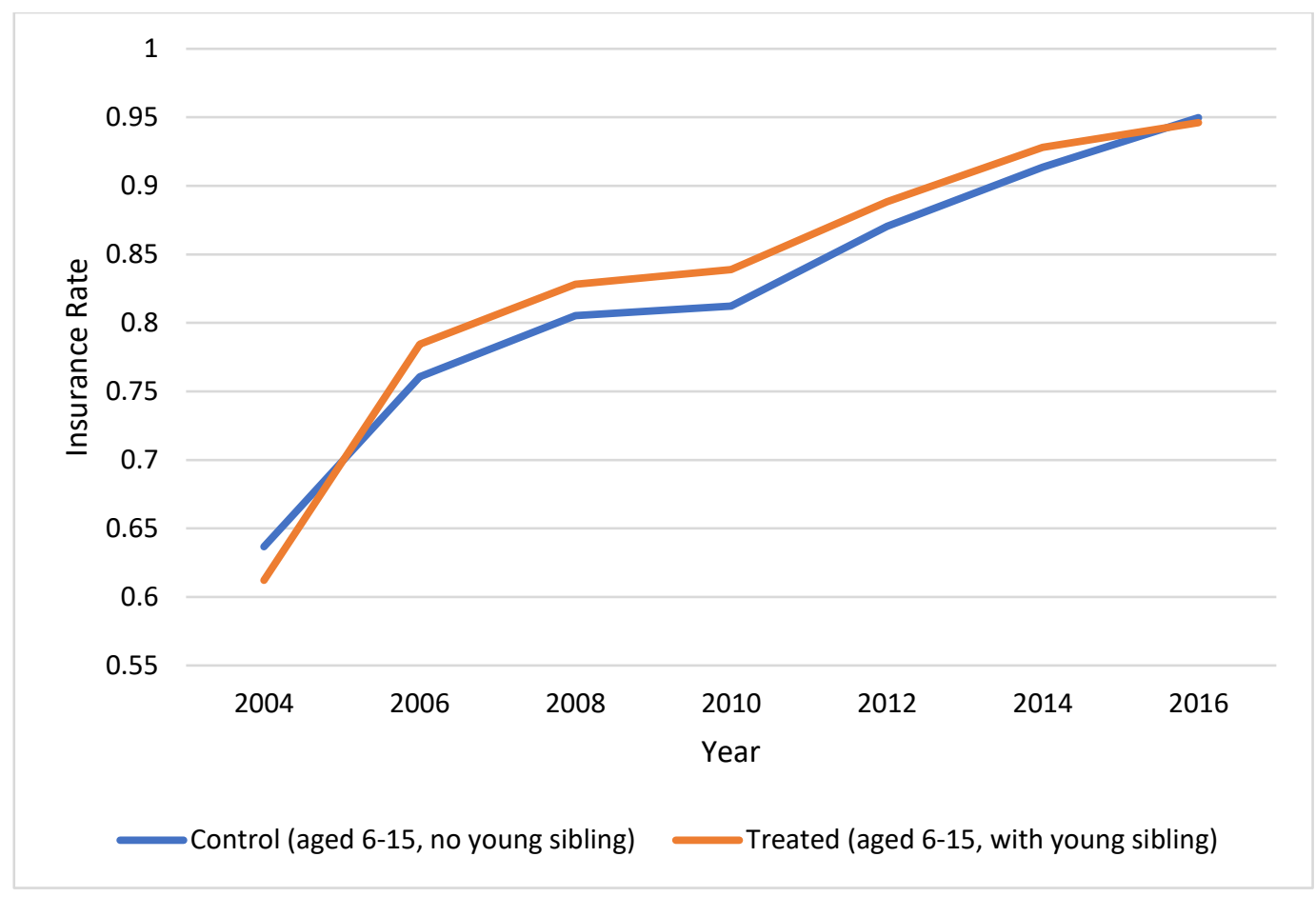

Figure 5. 10 The Reported Health Insurance Status of Two Groups of Children

Indeed, I find that the FHI policy targeting young children can have a spill over effect on insurance coverage of older children. In Table 5.37, Column 1, the DD estimate shows that living with a targeted sibling can increase the health insurance rate by 3.9 percentage points (statistically significantly at 1\%). I argue that providing free insurance for young children can free available household resources so that parents can purchase health insurance for other children to maintain parity within the family.

Since the spill over effect on insurance status is much higher than the persistent impact reported in previous section, a policy that subsidizes premiums (has wealth effects) might have more pronounced impact than the one that provides information on the benefits of health insurance (awareness effects). Our finding aligns with the results of previous experiments that examined the effects of information and premium subsidy on enrolment in voluntary health insurance in Vietnam. Specifically, A. Wagstaff, Nguyen, et al. (2016) found that the information brochure and a 25 percent premium subsidy had a negligible, insignificant effect on enrolment. Other studies suggest that higher rates of subsidization can yield higher impact on health insurance purchase; the authors argue that subsidizing 25 percent of insurance premiums might be insufficient to produce a significant impact. Not only 
do my results show that full subsidization substantially increases the coverage of beneficiaries (direct effect), but benefits can spill over to other household members.

The estimates reported in Table 5.37, Columns 3 and 4, show that the spill over effects on insurance coverage are more pronounced on children living in disadvantaged areas. Compared with urban residents, the policy impact on the insurance rate of rural children is statistically significantly greater by five percentage points since the policy change. Similarly, children in the Northern Midland and Mountainous Areas and the Central Highlands, two disadvantaged regions, tend to have better coverage when living with targeted siblings than those in other regions. Thus, it is apparent that the FHI policy for young children is more effective in increasing voluntary health insurance enrolment than an information campaign or programmes that provide partial premium subsidy, especially in rural and disadvantaged regions.

\subsubsection{Healthcare utilization}

Unlike previous results that show the FHI policy has a positive impact on both health insurance status and healthcare use among children under six, I find that the increased coverage because of living with under-six sibling does not translate into higher health utilization among children aged six to 15. Figure 5.11 illustrates an identical pattern of reported health visits in the last 12 months among children in the treated and control groups. I also find no difference in outpatient visit or inpatient admissions of these children.

Indeed, the DD estimates in Tables 5.38, 5.39, and 5.40 consistently show that the FHI policy for children under six children has no spill over effect on the probability of visiting health facilities or outpatient and inpatient use (the effects are small and not statistically different from zero). It is noteworthy that the results of a Poisson regression are consistent with our OLS estimates on the effects on the number of 


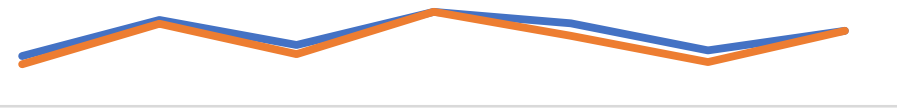

0

$\begin{array}{ccccccc}2002 & 2004 \quad 2006 \quad 2008 \quad 2010 \quad 2012 & 2014 & 2016 \\ \text { Year }\end{array}$

Control (aged 6-15, no young sibling)

Treated (aged 6-15, with young sibling)

Figure 5. 11 The Health Visits of Two Groups of Children in the Last 12 Months

outpatient and inpatient visits. Without the increase in healthcare use, I argue that the increase in coverage of older children is mainly driven by parents' desire to maintain parity among their children, not by the adverse selection related to voluntary insurance.

\subsubsection{Healthcare expenditure}

Despite the improving health insurance status of children aged six to 15 who live with eligible siblings, the FHI policy tends to have no effect on out-of-pocket health spending. Figure 5.12 shows that there is no difference in the total health expenditure of the treated and control groups over time. I also find no difference in spending for outpatient and inpatient care among these children.

The regression results report no spill over impact of FHI policy on healthcare expenditure among untargeted children. The DD estimates in Tables 5.41 and 5.42 show a reduction in total expenditure and outpatient spending by 1.0 and 1.7 percent, respectively. However, these estimates are statistically insignificant at $10 \%$. 


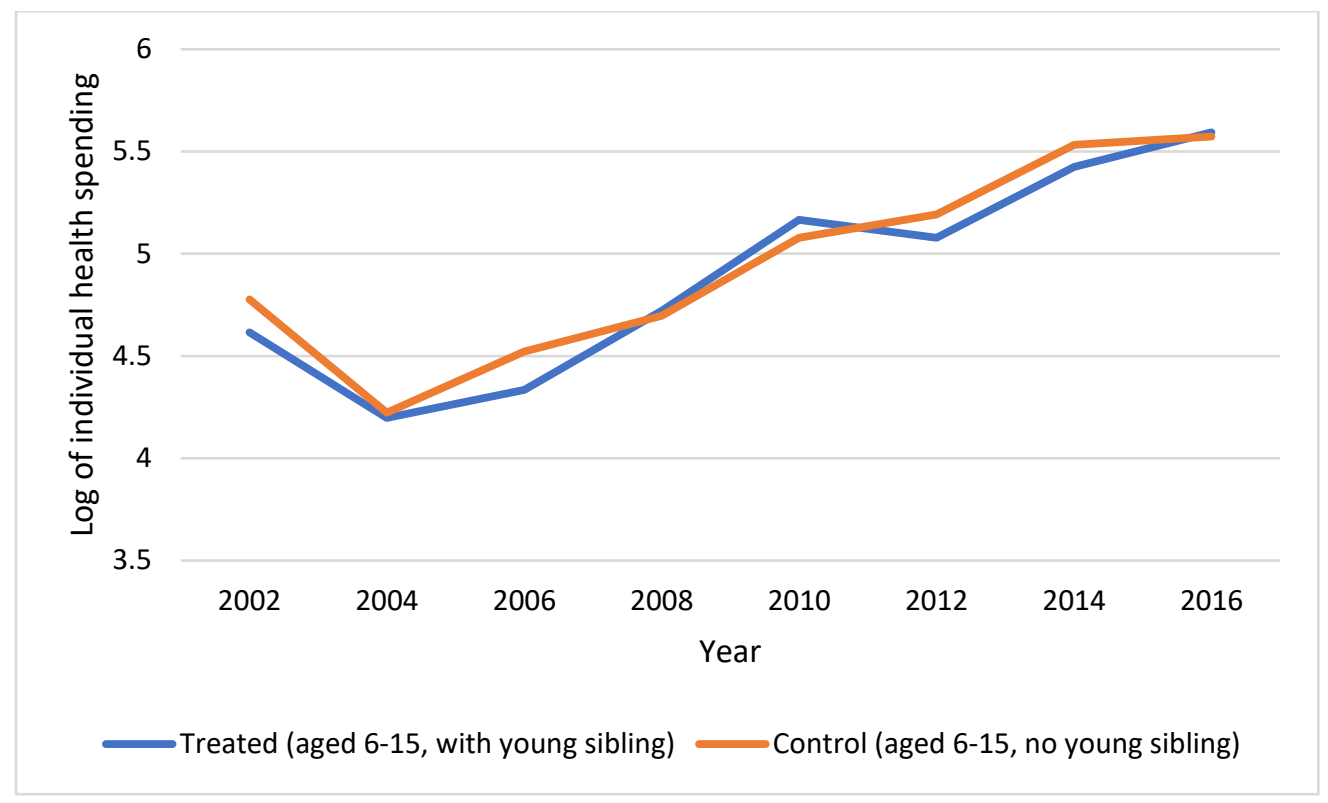

Figure 5. 12 The Out-of-Pocket Health Expenditure for Two Groups of Children

Similarly, the results in Table 5.43 show a negligible and insignificant impact of the policy on inpatient payment. The lack of impact on out-of-pocket payments is likely the result of the low utilization reported in the previous section. My results are consistent with the results from a Tobit model suggesting that the OLS estimates are insensitive to functional form.

\subsection{Health status}

I find that the FHI policy for young children might improve the health status of untargeted children in the household. Figures 5.13 and 5.14 both indicate that children living with young siblings are less likely to report having an illness in the last 30 days and past 12 months than the control group. The differences in morbidity rates of these children tend to widen after the policy change. Despite having higher insurance rates than the control group, treated children seem also to have better health outcomes. This evidence further strengthens our argument of no adverse selection into enrolment. 


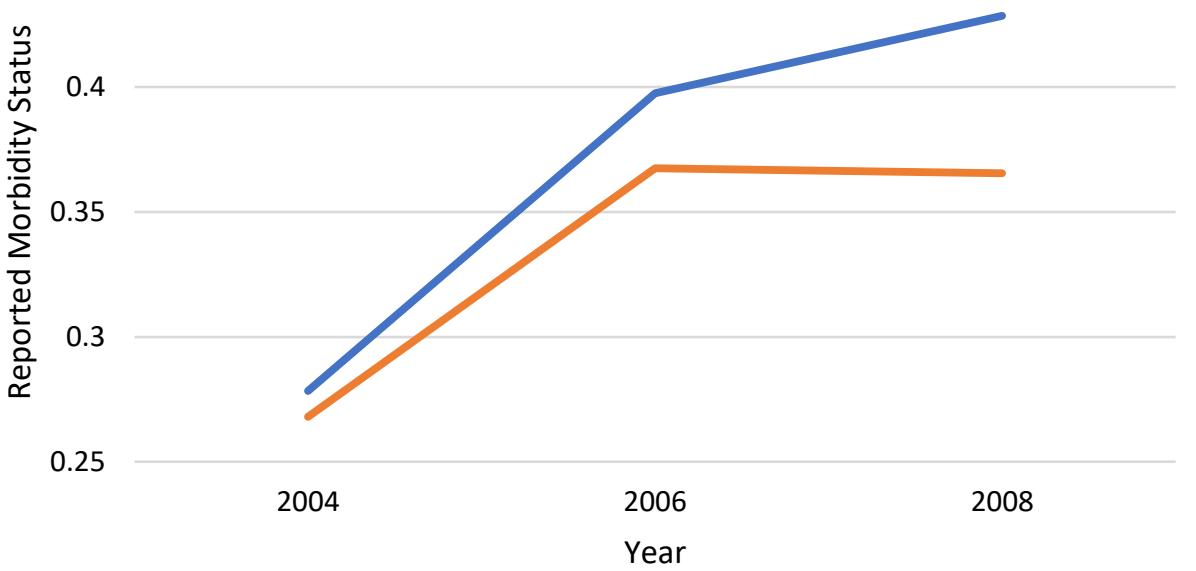

Control (aged 6-15, no young sibling) —Treated (aged 6-15, with young sibling)

Figure 5. 13 The Last Month Morbidity Rate for Two Groups of Children

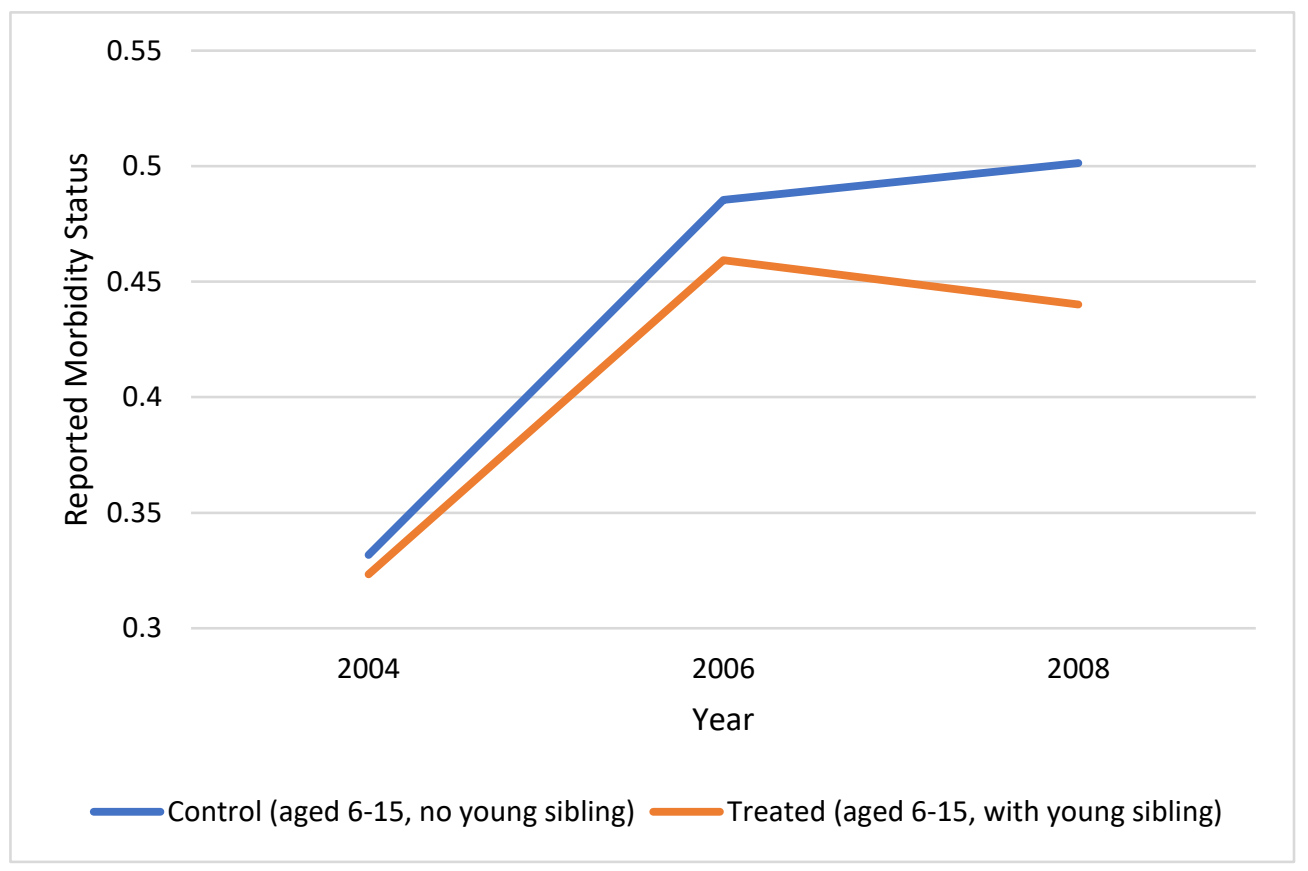

Figure 5. 14 The Last 12 Month Morbidity Rate for Two Groups of Children

In Tables 5.44 and 5.45, Column 1, the DD estimates consistently show that providing FHI for young children reduces both measures of morbidity status of older children by about two percentage points. However, the estimates are statistically insignificant at $10 \%$. I find that the spill over effect on morbidity rates is not uniform over time nor across regions. In Tables 5.44 and 5.45, Column 2, the impact was more pronounced and statistically significant in 2008, 
which indicates that the policy might take meaningful effect three years after implementation. Similarly, Tables 5.44 and 5.45, Column 3, show that the policy impact on the reported morbidity rates among rural children are statistically significantly lower than their urban peers by over four percentage points. Noticeably, the policy has greater effects on children in wealthy regions, such as the Red River Delta and the south-east than on those in the Central Highlands by eight and 14 percentage points, respectively. Although children in the Central Highlands tend to have better insurance status because of the policy change, I am unable to link this increase in coverage to better morbidity status because of the lack of healthcare utilization.

\subsection{Spill Over Effects on Other Individual and Household Outcomes}

\subsubsection{Individual education expenditure}

Using a panel dataset from the VLSS rounds 1992/93 and 1997/98, a previous study showed that voluntary health insurance (VHI) has a positive impact on household educational expenditure (A. Wagstaff \& Pradhan, 2005). The authors argue that VHI can reduce a household's financial risk of illness, thereby increasing its expenditure for other purposes, including the investment in education. Using the same argument, I am interested in whether providing FHI for young children can increase a household's spending on education for older children. Since the policy has been implemented nationally, my analysis is not susceptible to the selection bias attached to studies on VHI. Thus, my DD estimates of the average treatment effects of the FHI policy are unbiased (so long as the treatment is randomly assigned, the average treatment on the treated, ATT, is equal to the average treatment on the untreated, ATU). 


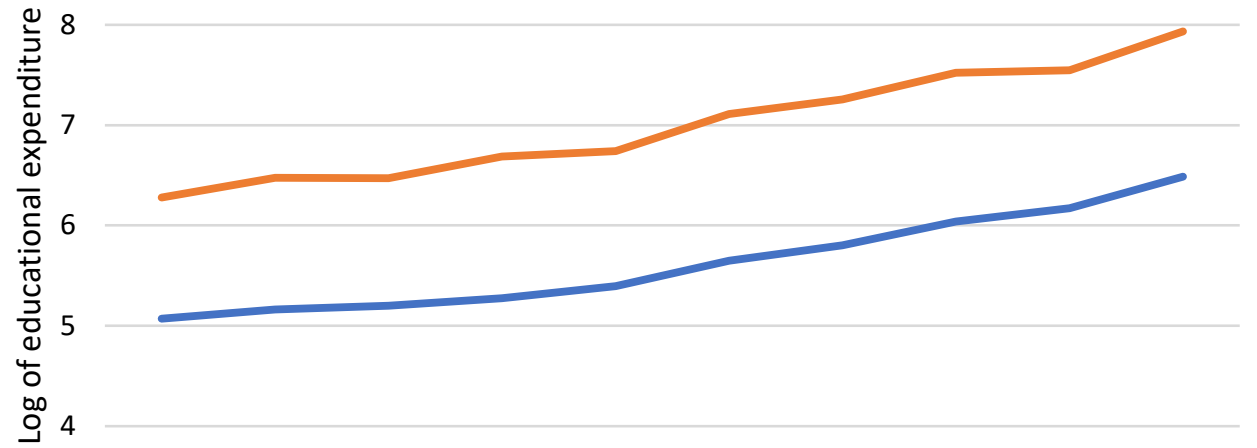

3

$\begin{array}{rrrrrrrrrr}6 & 7 & 8 & 9 & 10 & 11 & 12 & 13 & 14 & 15\end{array}$

Control (No Exposure) $\longrightarrow$ Treated (With Exposure)

Figure 5. 15 Individual Educational Expenditure for Two Family Groups

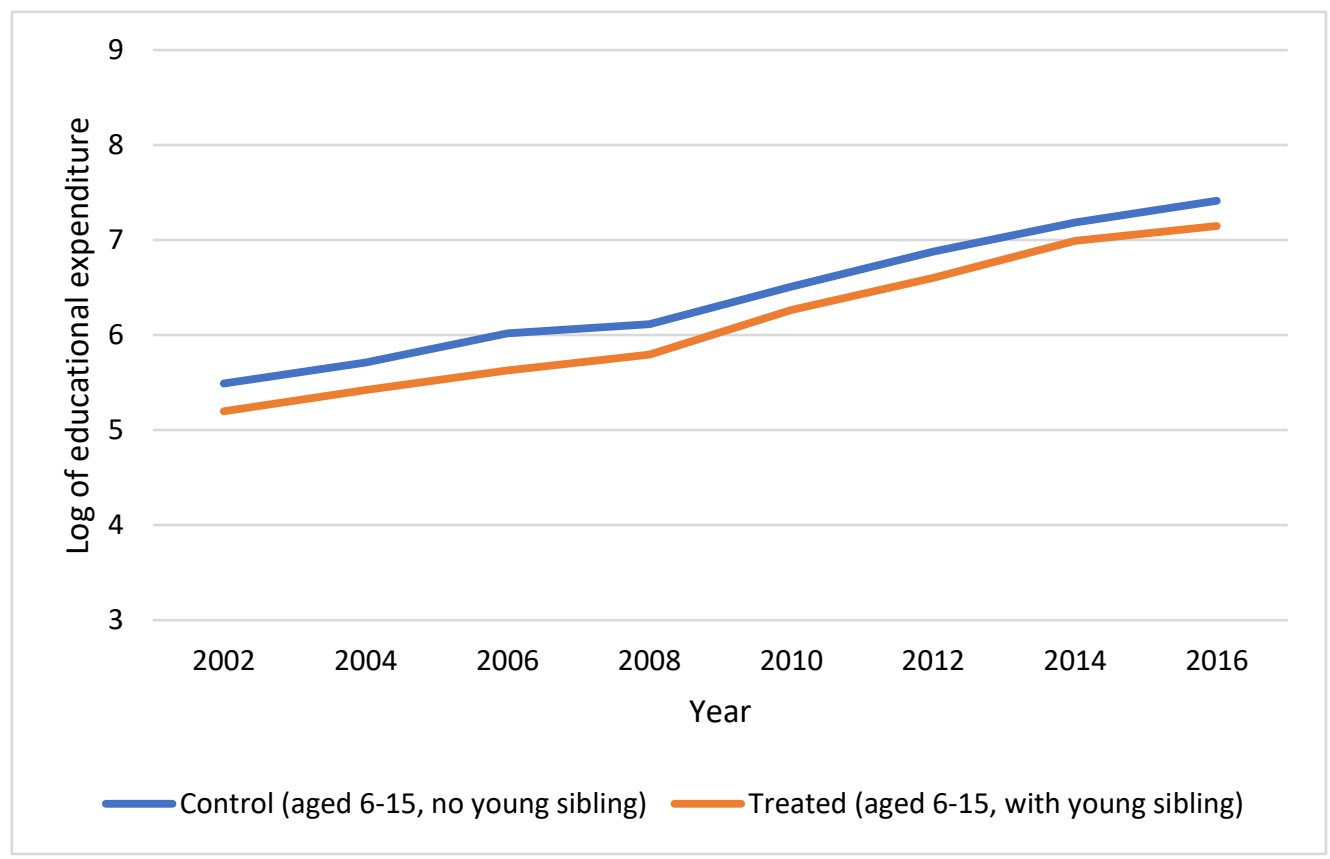

Figure 5. 16 Individual Educational Expenditure with Different-Aged Children

It is notable that the FHI policy can affect older children's education expenditure through two channels: the persistent effect because of their exposure to the policy at a young age; and the spill over effect because of living with a targeted sibling. Figure 5.15 shows educational spending tends to increase as children get older. The spending gap between children in the treated and control groups can be either attributed to the time trend in educational 
investment or previous exposure to $\mathrm{FHI}$ at a young age. On the other hand, Figure 5.16 shows that education expenditure on children living with a younger sibling is generally lower than that of children in the control group. Also, there seems to be no abrupt change in the spending pattern of treated children following the policy change.

Our regression results show that $\mathrm{FHI}$ can affect educational spending on older children through their exposure to it at a young age, but not with the presence of a targeted sibling in the household. Table 5.46, Column 1, suggests that exposure to at least one year of FHI at a young age can increase future spending on education by $10 \%$ (statistically significant at $1 \%$ ). I find that the policy might have a disproportionate impact on spending across location. Since rural children received a lower level of investment in education than their urban peers, Table 5.46, Column 2, shows that the persistent effect of the FHI policy can narrow this spending gap. Across regions, the results in Table 5.46, Column 3, indicate that the prolonged effects are more pronounced on treated children in affluent areas such as the Red River Delta or the south-east than the rest of the country. In contrast, Table 5.47, Column 1, shows that the presence of an eligible sibling in the household has negligible, statistically insignificant impact on the education expenditure of older children.

\subsubsection{School attendance}

A previous study on the determinants of school attendance among children in Vietnam found that a lack of interest in studying led to poor performance in class; household poverty and parental education were the main factors contributing to early school drop-out (T. D. Le \& Tran, 2013). In this section, I add evidence to the debate on whether health insurance plays a role in school attendance among children aged six to 15 in Vietnam. As previously pointed out in the section 5.4.1, the FHI policy for children under six can affect schooling through two mechanisms. First, if the exposure to $\mathrm{FHI}$ at a young age makes these children healthier, this positive health impact can be beneficial to school performance. Second, the presence of a young sibling who is entitled to $\mathrm{FHI}$ in the household has a positive health effect on older children. Thus, the policy might also have spill over effects on schooling of untargeted children. 
Figure 5.17 shows that the school attendance rates tend to decline as children get older. The past entitlement to $\mathrm{FHI}$ seems to have larger impact on children younger than eight and older than 13. However, at this point I am unable to separate the effect of exposing to FHI at a young age to the time trend in education. Meanwhile, the effect of FHI policy on school attendance rates is visually displayed in Figure 5.18. Indeed, schooling rate of treated children increases, not immediately, but five years after the policy was implemented.

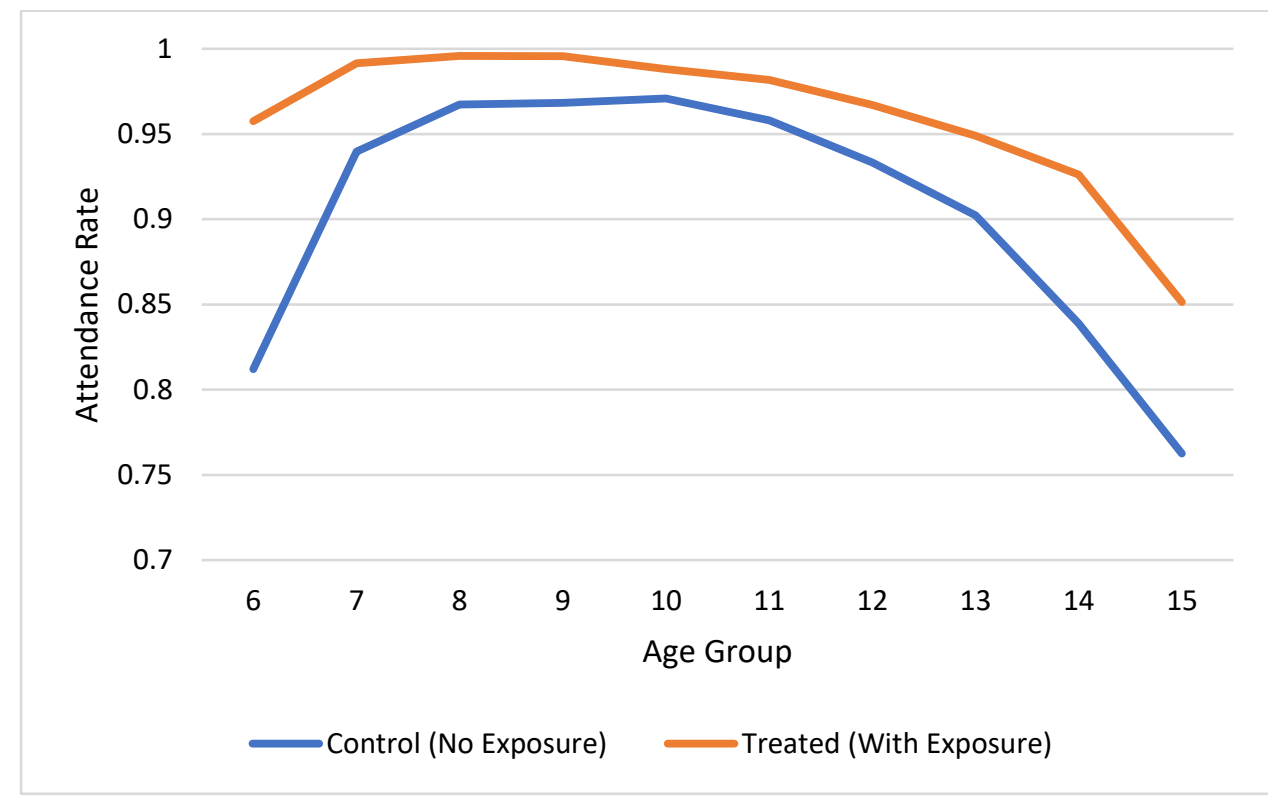

Figure 5. 17 The School Attendance Rate of Vietnamese Children by Age

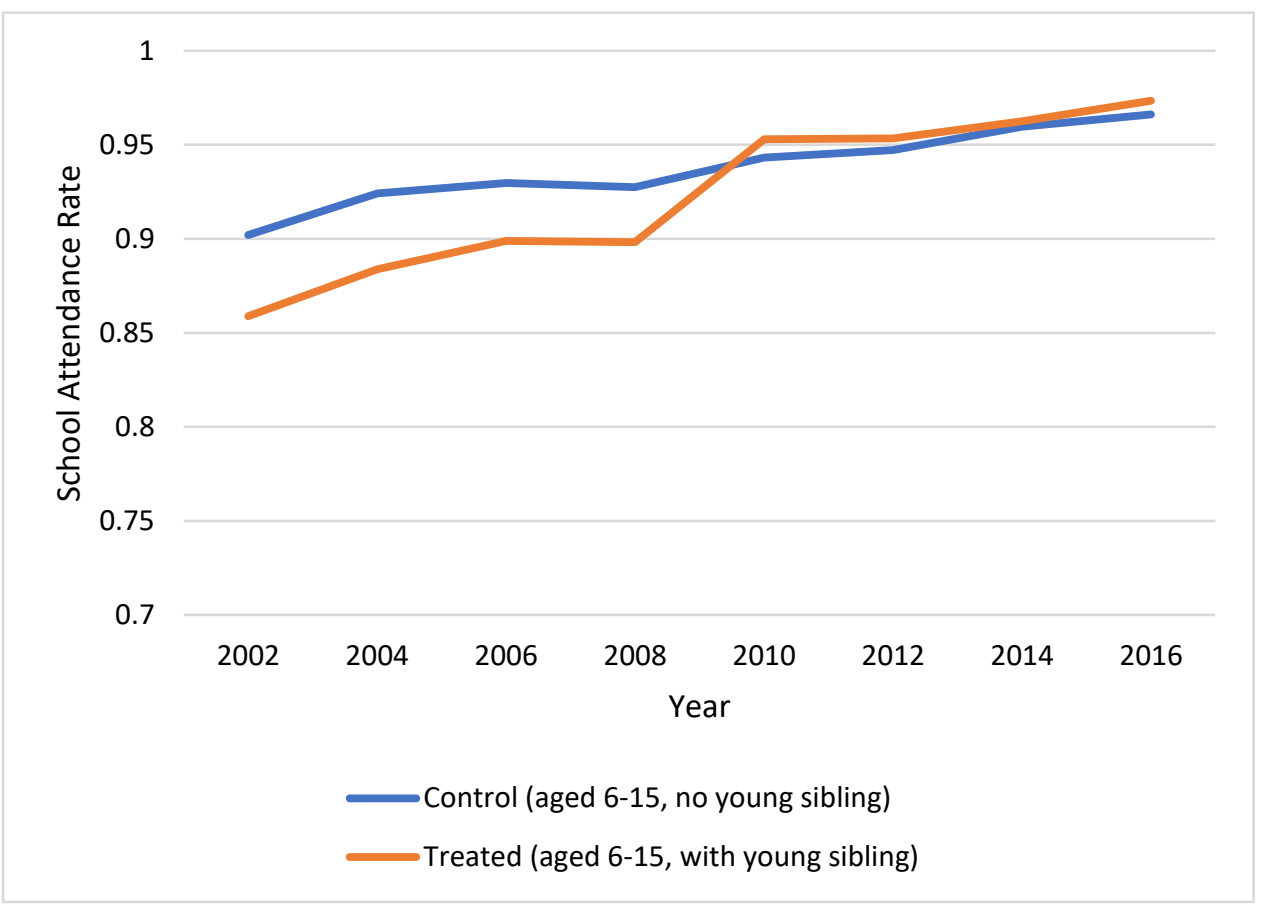

Figure 5. 18 School Attendance Rate of Vietnamese Children by Year 
Our regression results suggest that the FHI policy change might benefit children who live with a beneficiary, not those with past entitlement. Although Table 5.48 shows that exposure to $\mathrm{FHI}$ at a young age has positive and statistically significant impact on schooling, the effect is too negligible to prove any meaningful impact. In contrast, the DD estimates in Table 5.49 indicate that living with a targeted young sibling increases the school attendance rate of older children by 2.8 percentage points (statistically significant at $1 \%$ ).

I argue that the FHI policy might reduce the financial risk of illness so that parents can use the available sources (which would have been withheld otherwise) to send their children to school. Since young children can be healthier under the FHI policy (reported in section 5.1.4), older children who are less susceptible to infectious diseases in a household can perform well at school. I show that the provision of $\mathrm{FHI}$ for young children can have either a wealth or health effect on the education measures of older children. More generally, my finding supports the idea that the household environment is critical to the educational attainment of young children. From a policy perspective, my analysis shows that a programme aiming to promote child health can have impact beyond its target group.

\subsubsection{Household earnings}

In this section, I present evidence on the $\mathrm{FHI}$ impact on household wage income and per capita income. Recent studies provide mixed evidence on the impact of health policy on household labour supply. While Beuermann and Garzon (2016) show that intervention in Africa can increase parental household supply, N. Le et al. (2019) suggests that the free health insurance policy for the poor in Vietnam might reduce household working hours. This study complements these papers by examining whether the FHI policy for young children has any impact on household wages and income per capita. 


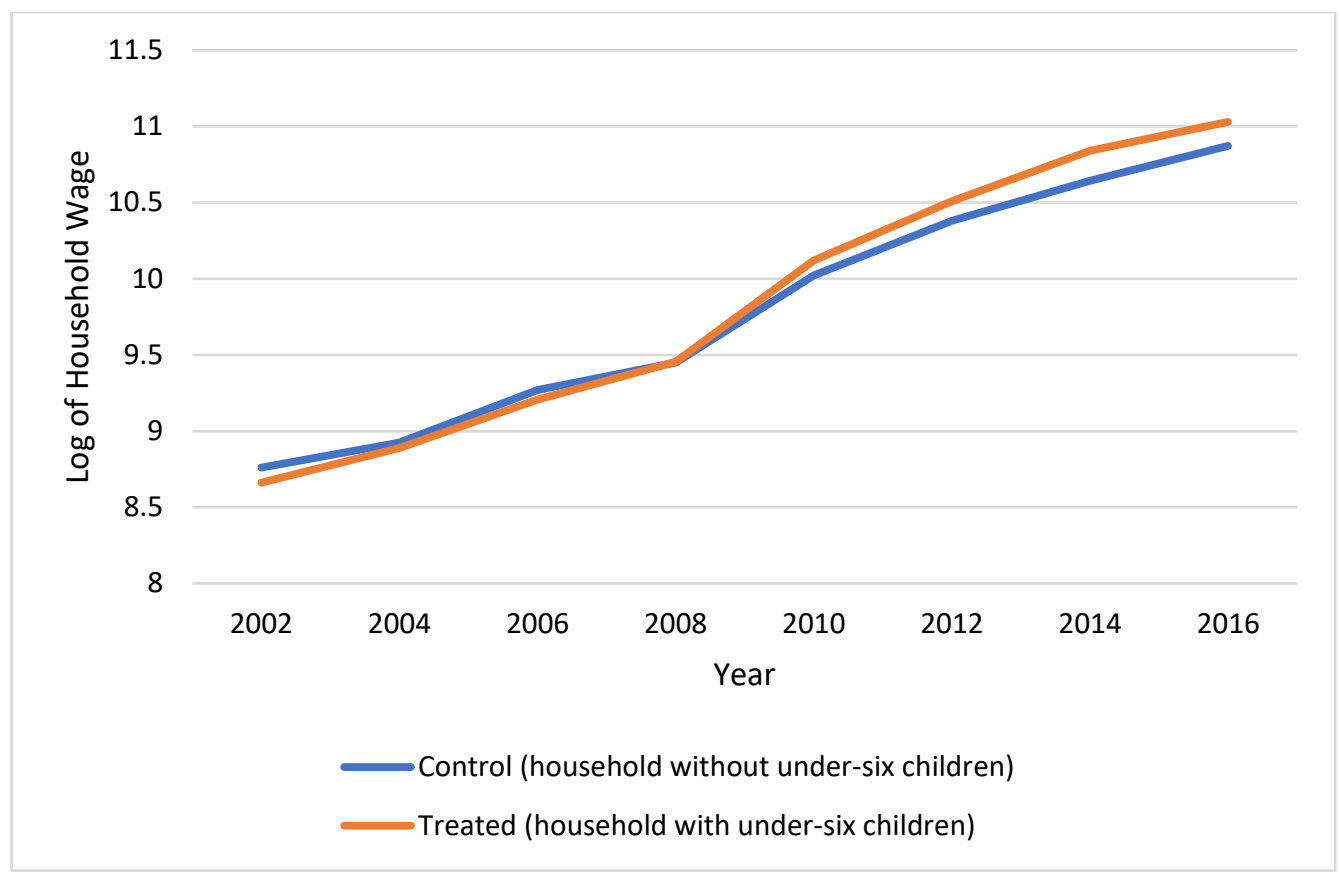

Figure 5. 19 Household Wage Income with Different Aged Children

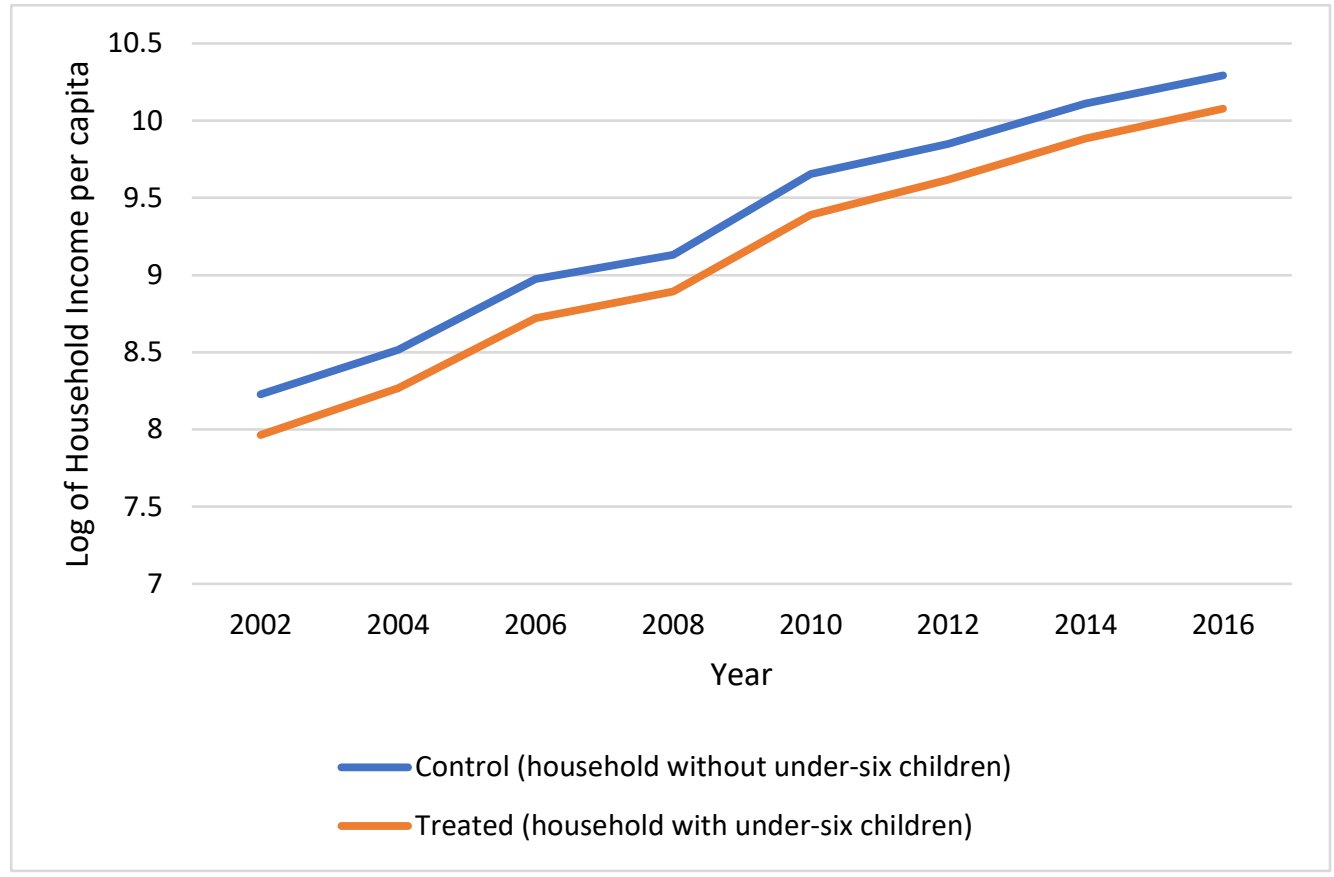

Figure 5. 20 Household Income Per Capita with Different Aged Children

Figure 5.19 shows that household wage income follows an upward trend in which a household with young children tends to earn more than one without young children five years after the policy change. Similarly, Figure 5.20 illustrates increased household income per capita though a typical treated household tends to earn less than the control group. These patterns of 
household earning are consistent with the economic development in Vietnam over the past 30 years.

My results show evidence of a positive impact of the FHI policy on household wages and income per capita. Table 5.50, Column 1 indicates that the policy can increase household wages of households with young children by 9.8 percent (statistically significant at $1 \%$ ). Since the estimates in Table 5.50, Column 2, demonstrate that the policy started having substantial impact after five years, it is relevant to use data covering a longer time period to study health insurance policy in Vietnam. The results in Table 5.50, Column 3 indicate that the policy has a favourable effect on the wage income of households in rural areas. Across regions, Table 5.50, Column 4, shows that the policy has most impact on household wages in the Red River Delta. Suppose that the policy change motivated parents to increase their working hours or to look for a new job, the larger effect on households in the Red River Delta, where the labour market is more robust, might be because of the flexibility to adjust for labour supply in the area. In contrast, the results in Table 5.51, Column 1 show a negligible impact of the FHI policy on income per capita. Compared with the control group, the income per capita in households with young children is 1.4 percent higher (statistically significant at $10 \%$ ). Since production costs become more expensive in the country, the increases in prices might offset any wage gain accrued from the policy.

\subsubsection{Household expenditure}

In this section, I provide evidence on the $\mathrm{FHI}$ impact on total household expenditure and various sub-aggregates of household expenditure, including healthcare and education expenditure. Previous studies showed that the voluntary health insurance policy in Vietnam can boast household nonmedical consumption using a panel dataset from VLSS 1992/93 and 1997/98 (A. Wagstaff \& Pradhan, 2005). In the same spirit, our analysis, which is not susceptible to selection bias inherent in VHI with the most recent dataset, can add to discussion on the impact of health insurance policy on household outcomes in Vietnam.

\subsubsection{Household expenditure per capita and per adult equivalent}

Figures 5.21 and 5.22 show an overall increase in both measures of household expenditure in which a typical household with young children tends to have lower consumption than the one 
without young children. It is noteworthy that the increase in household expenditure since 2010 might be because of the change in the VHLSS sample frame reflects a higher proportion of urban households. Column 1 in Table 5.52 and 5.53 shows that the policy has a negative impact on household consumption. However, I argue that the policy hardly has any meaningful impact on household expenditure because the size of the effect is negligible.

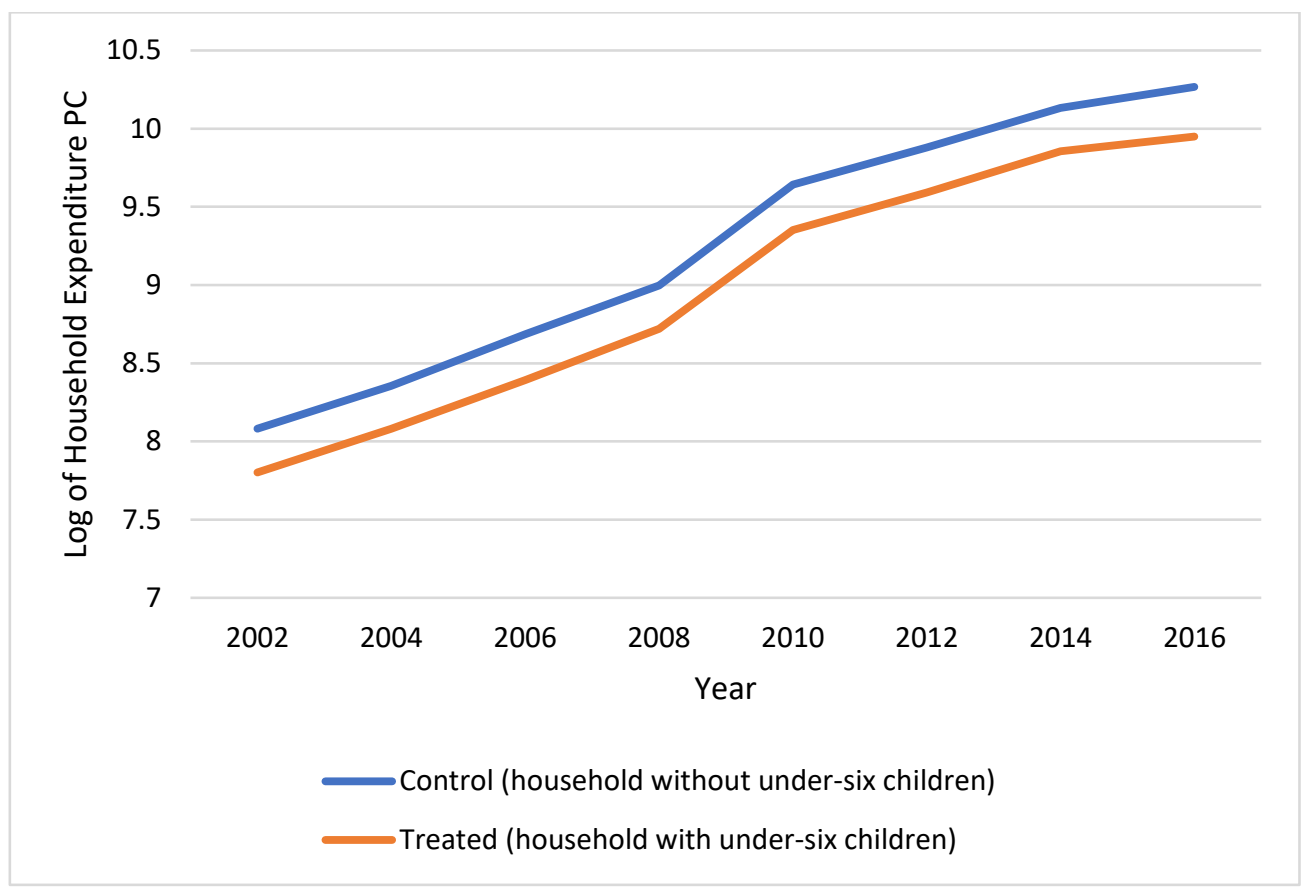

Figure 5. 21 Per Capita Household Expenditure Per Capita for Two Different Groups

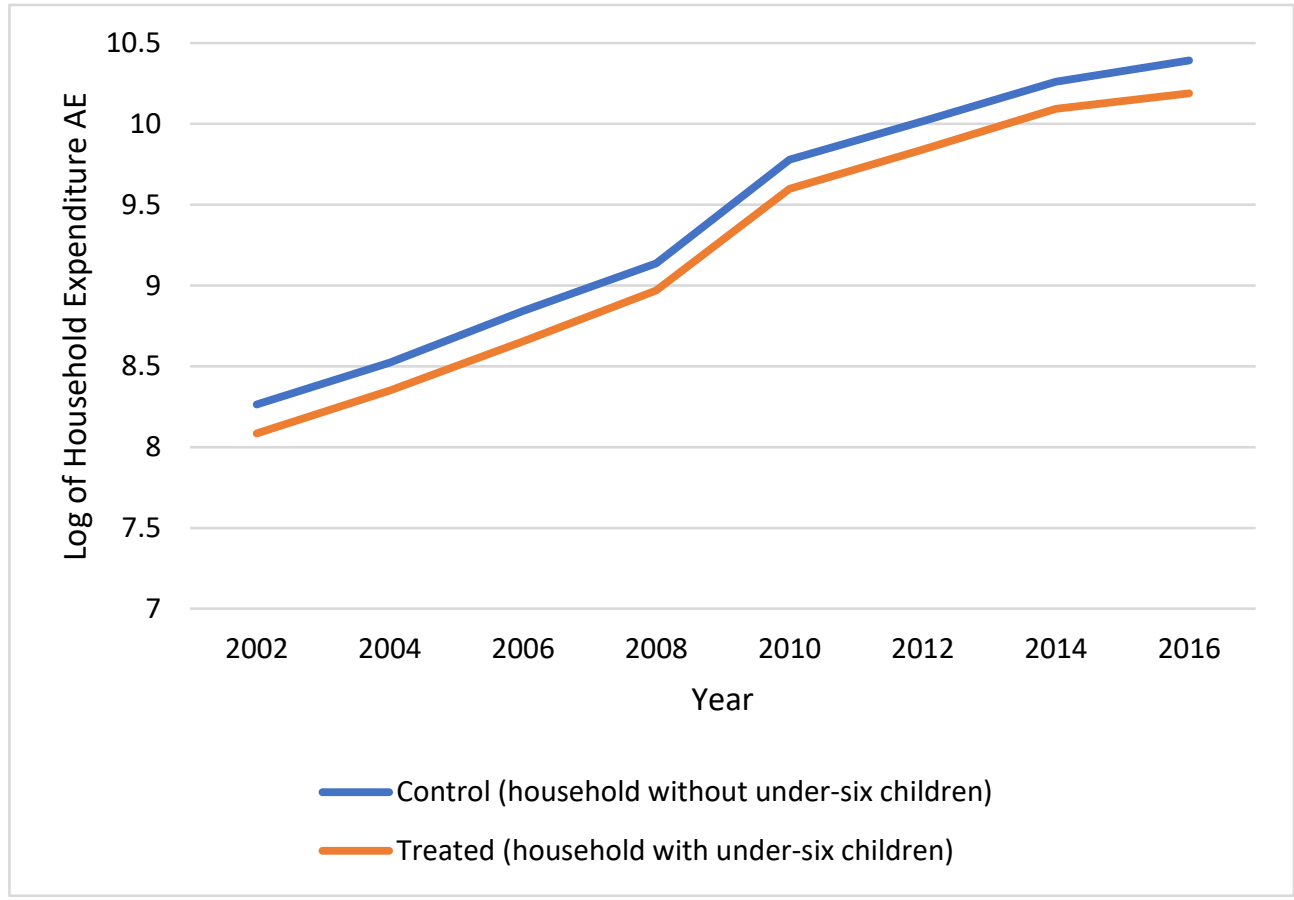

Figure 5. 22 Per Adult Equivalent Household Expenditure for Two Different Groups 
In contrast to a previous study that found a positive impact of $\mathrm{VHI}$ on education spending (A. Wagstaff \& Pradhan, 2005), our results show that the FHI policy decreases education expenditure of household with young children. Figure 5.23 below shows that, while investment in education tends to increase over time, treated households spend less on education than control group households. Without controlling for household characteristics that might affect education spending, it is unclear if the policy has any effect on this measure. The regression results in Table 5.54, Column 1 show that the policy decreases education spending by 10 percent (statistically significantly at $1 \%$ ). A Tobit regression produces similar results which indicates that the OLS estimates are robust. In Table 5.54 in Column 2 and 4, I find that the policy impact has been greater since 2010 and varies across locations. Compared with the Central Highlands, the policy has less negative impact on education spending in wealthy regions such as the Red River Delta and the south-east.

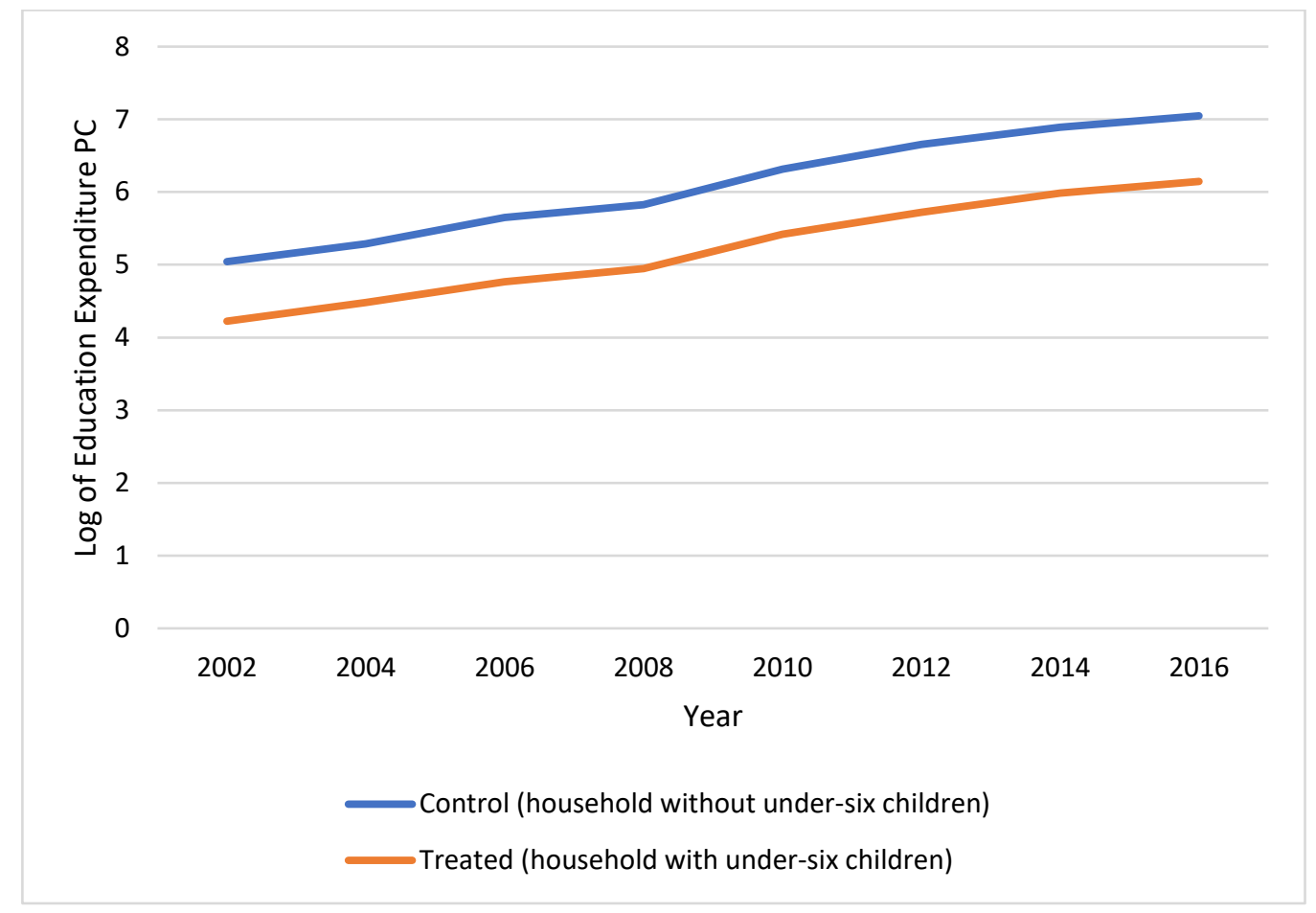

Figure 5. 23 Household Education Expenditure Per Capita for Two Different Households

I find no evidence on the policy effects on household healthcare expenditure and the probability of incurring catastrophic healthcare costs. Figures 5.24 and 5.25 display a contrasting picture of the patterns of healthcare spending in Vietnam. Though per capita out- 
of-pocket payments continue to rise, the likelihood of spending more than 10 percent of consumption for healthcare tends to decline over time (the same is true for the 25 and 40 percent thresholds). Perhaps strong economic growth over a long period of time has helped households absorb the increased healthcare costs. Since low-income households are most vulnerable to health shocks, the current pro-poor policies might protect them from catastrophic spending. However, the results in Table 5.55, Column 1, show that the policy had no significant aggregate effect on healthcare expenditure per capita. The findings consistently indicate that the policy change is unable to provide financial protection at all.

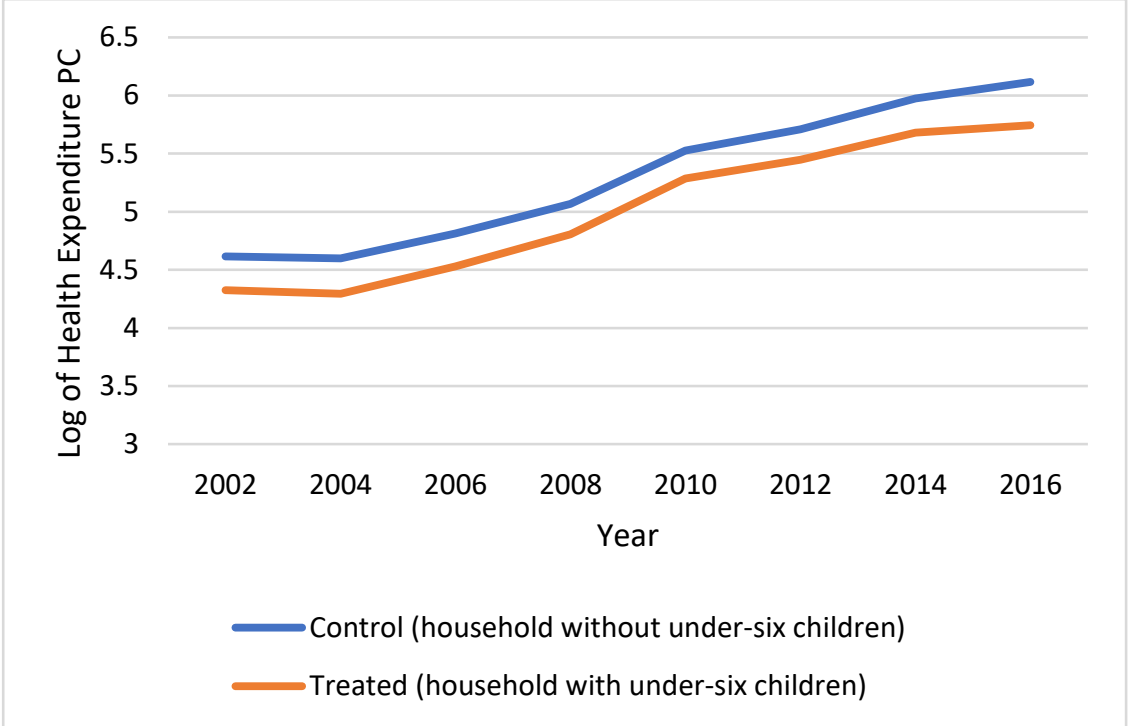

Figure 5. 24 Household Health Expenditure Per Capita for Two Different Households

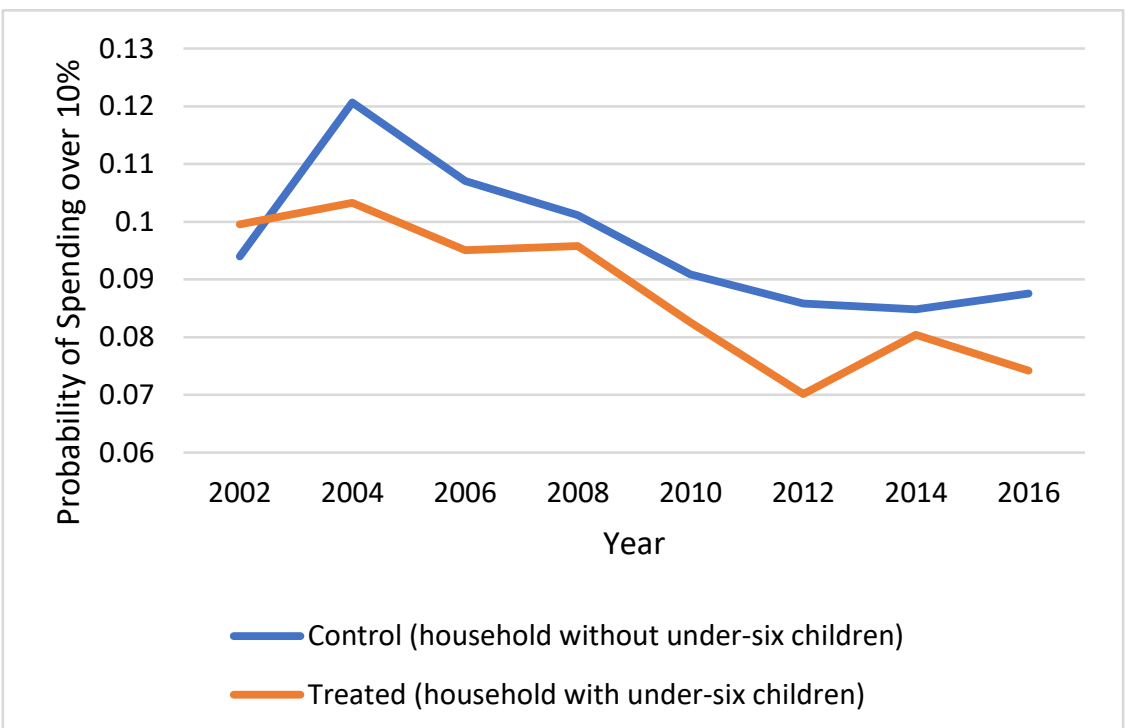

Figure 5. 25 The Probability of Spending over $10 \%$ of Consumption on Healthcare for Two Different Households 
Though the results in Table 5.55, Column 3, show no differential effects of spending between urban and rural areas, the estimates in Column 4 point to a significantly greater impact of FHI on households across regions in the country compared to the Central Highlands. The lower impact on healthcare spending in the Central Highlands can be because of to either low healthcare use or higher medical costs in other regions. Similarly, the estimates in Table 5.56, Column 1 suggest that FHI policy for young children has no overall effect on the probability of paying large portions of household consumption on healthcare. However, Table 5.56, Column 3, shows that the FHI policy significantly decreased the catastrophic expenditure incidence of rural households by 3.9 percentage points. This is consistent with the study of A. Wagstaff (2007b) that showed the vulnerability of urban households when facing health shocks. Moreover, the results in Table 5.56, Column 4, indicate that the impact is greater on households in South Vietnam than in the Central Highlands.

\subsubsection{Household health insurance coverage and calorie consumption}

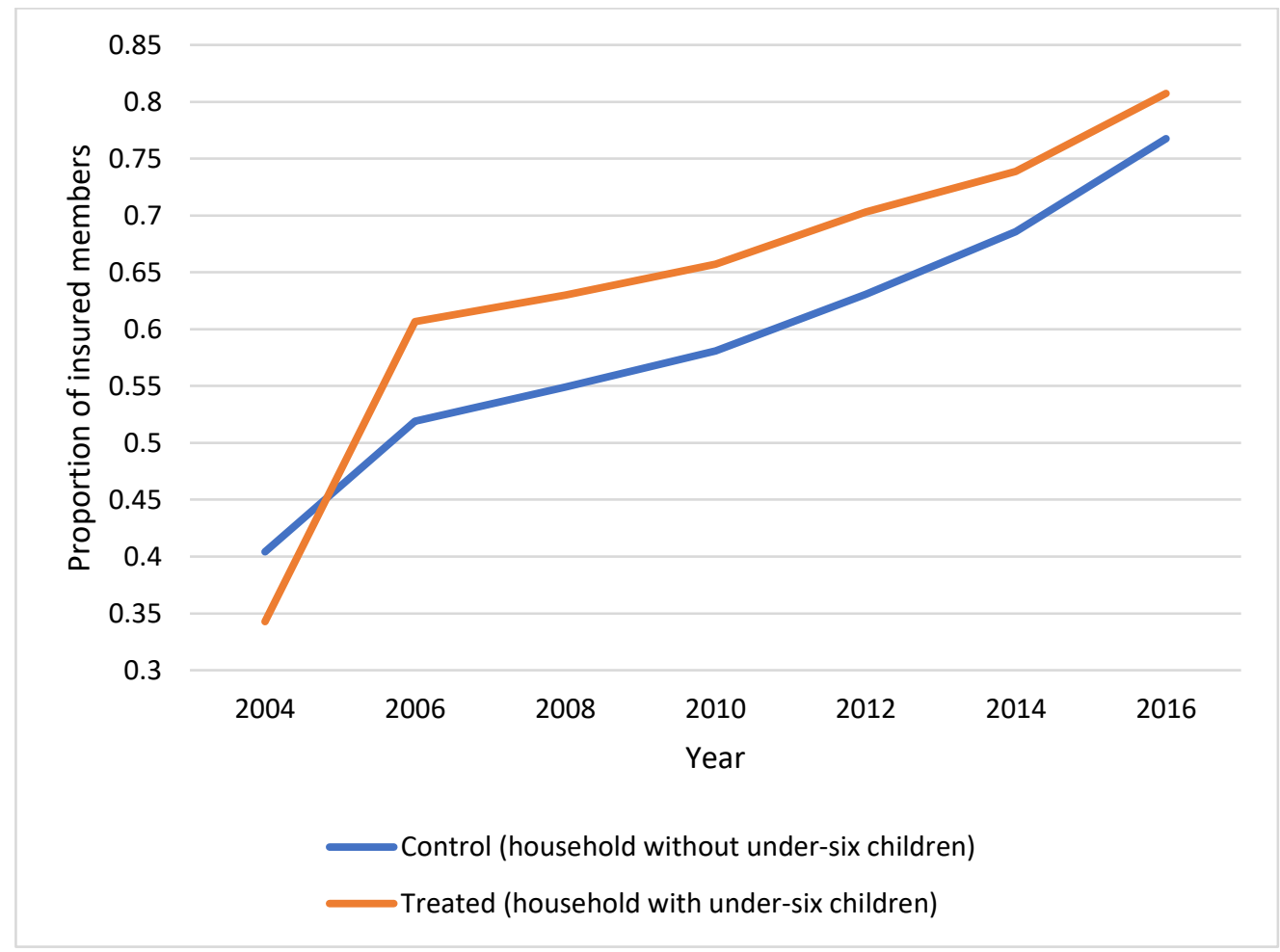

Figure 5. 26 Household Health Insurance Rate for Two Different Households

I previously reported that children aged six to 15 who live with younger siblings are more likely to have insurance than those without younger siblings. In this section, I provide more evidence of the spill over effect of the FHI policy on the insurance rate for other household 
members. Figure 5.26 shows that the proportion of household member with insurance increases substantially among household with young children. The estimates in Table 5.59, Column 1 indicate that the presence of targeted children in the household increases household coverage by 12 percentage points (statistically significantly at $1 \%$ ). This substantial effect size suggests that the benefit of this policy change might be extended to other family members. As previously reported, the direct and spill over effects on the individual insurance rate are 40 (reported in Table 5.1) and 3.9 (presented in Table 5.37) percentage points, respectively).

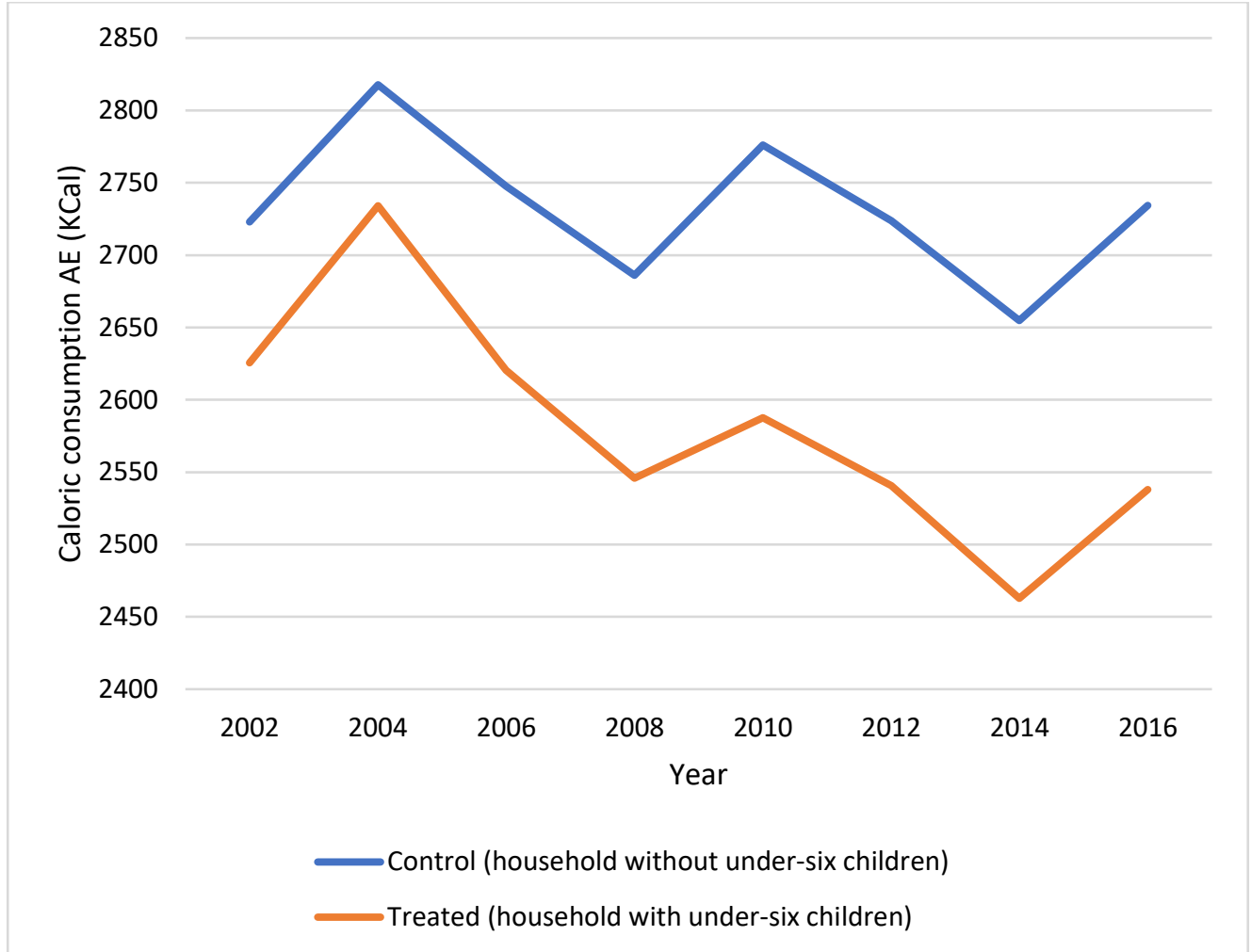

Figure 5. 27 The Household Calorie Consumption Per Adult Equivalent for Two Different Households

Meanwhile, I also find that $\mathrm{FHI}$ might have a negative impact on household calorie consumption. Figure 5.27 shows that calorie consumption per adult equivalent slightly decreased during the study period. Though the amount of calories consumed in a household with young children is lower than the control household, the gap between them widens over time. The DD estimates in Table 5.60, Column 1 suggest that the FHI policy decreases calorie consumption by $88 \mathrm{Kcal}$ (statistically significantly at $1 \%$ ). However, since this amount accounts for only four percent of the average calorie consumption, I argue that the size of the effect is insignificant in drawing a conclusion about the policy's impact. 


\section{Chapter 6 DISCUSSION AND CONCLUSION}

This section summarizes the main findings and discuss their implications in relation to existing literature. These raise some issues about social health insurance that are relevant for countries at similar levels of development. The chapter concludes with some suggestion for future research.

\subsection{Main Findings}

Table 6.1 shows that free health insurance policy for young children in Vietnam tended to have noticeable direct impacts on various outcomes of these young children and moderate to negligible persistent and spillover effects on older children. Meanwhile, the results in Table 6.2 suggest that this policy might not have meaningful influence on other household outcomes, such as total income or caloric intake. Below I will discuss the results and propose some explanations for these estimates.

Health insurance status. I find that the policy succeeded in improving the health insurance status of young children in Vietnam. Under the Children Protection Law (25/2004/QH11) of 2004, children under six in Vietnam are entitled to free health care since 1 January 2005. My results show that since the policy change, children under six were 40 percentage points more likely to report having health insurance than children aged six to ten. This is a remarkable achievement for a developing country where catastrophic healthcare expenses can push people into destitution.

Despite the expansion in health insurance coverage, existing studies show that the insured tend not to use FHI when seeking care (Schmidt et al., 2012; Shieh et al., 2013). This can be for several reasons. First, beneficiaries might be not interested in seeking care in overcrowded public hospitals with long waiting lines; they opt for more convenient options like visiting private clinics run by doctors who work after hours, or they purchase drugs at pharmacy without prescription. This makes it harder to contain private healthcare costs because payments to private doctors and drugstores are not reimbursed under SHI in Vietnam. 
Table 6.1 The effects of free health insurance policy for young children on individual outcomes

\begin{tabular}{|c|c|c|c|c|}
\hline & Direct effects & Persis & ects & Spillover effects \\
\hline $\begin{array}{l}\text { Outcome } \\
\text { variables }\end{array}$ & & $\begin{array}{l}\text { Exposure } \\
\text { (Yes/No) }\end{array}$ & $\begin{array}{l}\text { Years of } \\
\text { exposure }\end{array}$ & \\
\hline $\begin{array}{l}\text { Insurance } \\
\text { status }\end{array}$ & $\begin{array}{c}0.410 * * * \\
(0.013)\end{array}$ & $\begin{array}{c}0.009 \\
(0.007)\end{array}$ & $\begin{array}{l}0.003^{*} \\
(0.002)\end{array}$ & $\begin{array}{c}0.039 * * * \\
(0.010)\end{array}$ \\
\hline $\begin{array}{l}\text { Health visit } \\
\text { (Yes/No) }\end{array}$ & $\begin{array}{c}0.081^{* * *} \\
(0.012)\end{array}$ & $\begin{array}{c}0.023^{* * *} \\
(0.008) \\
\end{array}$ & $\begin{array}{c}0.000 \\
(0.002)\end{array}$ & $\begin{array}{c}-0.011 \\
(0.010) \\
\end{array}$ \\
\hline $\begin{array}{l}\text { Outpatient visit } \\
\text { (times) }\end{array}$ & $\begin{array}{c}0.291 * * * \\
(0.061)\end{array}$ & $\begin{array}{c}0.092 * * * \\
(0.030)\end{array}$ & $\begin{array}{l}-0.001 \\
(0.009)\end{array}$ & $\begin{array}{l}-0.012 \\
(0.050)\end{array}$ \\
\hline $\begin{array}{l}\text { Inpatient visit } \\
\text { (times) }\end{array}$ & $\begin{array}{c}0.023 * * * \\
(0.011) \\
\end{array}$ & $\begin{array}{c}0.009 \\
(0.007) \\
\end{array}$ & $\begin{array}{l}-0.000 \\
(0.002) \\
\end{array}$ & $\begin{array}{c}0.000 \\
(0.009)\end{array}$ \\
\hline $\begin{array}{l}\text { Health } \\
\text { expenditure } \\
\text { (log) }\end{array}$ & $\begin{array}{c}0.340 * * * \\
(0.030)\end{array}$ & $\begin{array}{c}0.131^{* * *} \\
(0.040)\end{array}$ & $\begin{array}{c}0.036 * * * \\
(0.009)\end{array}$ & $\begin{array}{l}-0.010 \\
(0.031)\end{array}$ \\
\hline $\begin{array}{l}\text { Outpatient } \\
\text { expenditure }\end{array}$ & $\begin{array}{c}0.331 * * * \\
(0.030)\end{array}$ & $\begin{array}{c}0.110 * * * \\
(0.030)\end{array}$ & $\begin{array}{c}0.037^{* * *} \\
(0.008)\end{array}$ & $\begin{array}{c}0.048 \\
(0.060)\end{array}$ \\
\hline $\begin{array}{l}\text { Inpatient } \\
\text { expenditure }\end{array}$ & $\begin{array}{c}0.061 * * * \\
(0.021)\end{array}$ & $\begin{array}{c}0.030 \\
(0.020)\end{array}$ & $\begin{array}{l}-0.001 \\
(0.005)\end{array}$ & $\begin{array}{l}-0.029 \\
(0.040)\end{array}$ \\
\hline $\begin{array}{l}\text { Morbidity } \\
\text { status (last } 30 \\
\text { days) }\end{array}$ & $\begin{array}{c}-0.028^{* * *} \\
(0.020)\end{array}$ & $\begin{array}{c}0.044 \\
(0.030)\end{array}$ & $\begin{array}{l}-0.002 \\
(0.010)\end{array}$ & $\begin{array}{c}-0.023 \\
(0.010)\end{array}$ \\
\hline $\begin{array}{l}\text { Morbidity } \\
\text { status (last } \\
\text { year) }\end{array}$ & $\begin{array}{l}-0.013 \\
(0.010)\end{array}$ & $\begin{array}{c}0.032 \\
(0.030)\end{array}$ & $\begin{array}{c}0.002 \\
(0.010)\end{array}$ & $\begin{array}{c}-0.022 \\
(0.010)\end{array}$ \\
\hline $\begin{array}{l}\text { Education } \\
\text { expenditure } \\
(\log )\end{array}$ & & $\begin{array}{c}0.101^{* * *} \\
(0.030)\end{array}$ & $\begin{array}{l}-0.001 \\
(0.008)\end{array}$ & $\begin{array}{c}0.000 \\
(0.010)\end{array}$ \\
\hline $\begin{array}{l}\text { School } \\
\text { attendance } \\
\text { (Yes/No) }\end{array}$ & & $\begin{array}{c}0.001 \\
(0.005)\end{array}$ & $\begin{array}{c}0.002 * * \\
(0.001)\end{array}$ & $\begin{array}{c}0.028^{* * *} \\
(0.004)\end{array}$ \\
\hline
\end{tabular}

Notes to Table 6.1: The regression results reported in the following tables are adjusted for year fixed effects. I also include control variables for the characteristics of children (age, gender, ethnicity) and of the household head (age, gender, education level). I also control for household characteristics such as rural/urban and regions; ${ }^{*}=p<0.05 ;{ }^{* *}=p<0.01$; and ${ }^{* * *}=p<0.001$. Standard errors are in parentheses. 
Table 6.2 The spillover effects of free health insurance policy for young children on household outcomes

\begin{tabular}{|l|c|}
\hline \multicolumn{1}{|c|}{ Outcome variables } & Marginal effects \\
\hline Income wage & $0.098^{* * *}$ \\
& $(0.021)$ \\
\hline Income per capita & $0.014^{*}$ \\
& $(0.009)$ \\
\hline Expenditure per capita & $-0.013^{*}$ \\
& $(0.007)$ \\
\hline Expenditure per adult equivalent & -0.008 \\
& $(0.007)$ \\
\hline Education expenditure per capita & $-0.100 * * *$ \\
& $(0.020)$ \\
\hline Catastrophic health expenditure $(>10 \%$ of & 0.004 \\
total household expenditure) & $(0.007)$ \\
\hline Catastrophic health expenditure $(>25 \%$ of & 0.004 \\
total household expenditure) & $(0.007)$ \\
\hline Catastrophic health expenditure $(>40 \%$ of & 0.004 \\
total household expenditure) & $(0.007)$ \\
\hline
\end{tabular}

Notes to Table 6.2: The regression results reported in the following tables are adjusted for year fixed effects. I include control variables for the characteristics of the household head (age, gender, education level). I also control for household characteristics such as rural/urban and regions; ${ }^{*}=p<$ $0.05 ; * *=p<0.01 ;$ and ${ }^{* * *}=p<0.001$. Standard errors are in parentheses.

Second, the complicated paperwork to use health insurance at public hospitals can turn the insured away. This is particularly relevant to people in rural areas whose low educational level is the main barrier preventing them from using FHI. Indeed, public hospitals in Vietnam are always full of busy people; patients and their caregivers might receive little information or help from staff. Previous studies raised the issue of the stigma of using the free healthcare card for the poor because the insured might be discriminated against $(\mathrm{H}$. T. Dao, Waters, \& Le, 2008). Fortunately, the government promptly solved this problem by using the same health insurance card for everyone.

Third, the insured might bypass primary facilities without formal referral to seek treatment at higher-level hospitals (P. Le et al., 2014; Lee, Oh, Hoang, Moon, \& Subramanian, 2019). This practice is common in Vietnam because lower-level hospitals in Vietnam are perceived as incapable of providing good quality care. When patients feel that the treatment is not helpful and are unable to get a formal referral, they are likely to seek care directly at tertiary hospitals 
and forgo the benefits of FHI. Not only does this increase private spending, but it also puts more pressure on the country's already overcrowded central hospitals. Though Vietnam has been trying to expand insurance coverage, the government should, at the same time, invest in building more hospitals and increase the number of beds and health workers.

The estimations also present evidence of persistent and spill over effects of the policy on the coverage of older children. It is likely that past entitlement to $\mathrm{FHI}$ and the presence of eligible young children in the households increases the insurance enrolment among older children. Moreover, I find that FHI can substantially increase the proportion of the insured in a household, which implies that the policy might also affect adults. It should be noted that the government has struggled to increase coverage among the uninsured in the informal sector because information campaigns and high premiums might fail to motivate these individuals (A. Wagstaff, Nguyen, et al., 2016). My results provide empirical evidence supporting the alternative approach: start promotion at a young age and within households to activate the intra-household allocation of resources. If this strategy is effective, we might find, in the future, an increase in coverage among informal workers who were previously exposed to FHI at a young age.

Healthcare utilization. Under the behavioural model of healthcare services used by Andersen (1995), gaining access to care is considered the primary determinant of healthcare utilization. Therefore, the provision of FHI might explain the positive impacts on health visit likelihood and outpatient care in this study. The results show that the likelihood of visiting health facilities among the beneficiaries significantly increased by eight percentage points compared with children aged six to ten. This increase accounts for 13 percent of the baseline health visit rate of 61 percent. Similarly, children under six had 0.29 more outpatient visits than older children; this increase equals 15 percent of the average visits of children in the control group. In contrast, I find limited effects of the policy on the number of inpatient visits and this is consistent with the finding that user fee exemption had no effect on inpatient use among rural children (Schmidt et al., 2012).

The increase in outpatient use among young children following the policy change seems to be a good thing because outpatient care provides, for example, vaccination at public facilities. Thus, FHI might be one factor that has a positive impact on the child mortality rate in Vietnam 
in the last decade. Previous studies suggest that the insured might switch from seeking care at private facilities and drugstores to public hospitals where expenses can be reimbursed under FHI (M. Palmer et al., 2014). Although our OLS estimates point to a statistically significant impact of the policy on inpatient use, the Poisson model shows otherwise. Since hospitalization is rare, the conclusion on the policy's impact on inpatient use seems to depend on the underlying assumptions of econometric models.

I further find that the policy change has persistent effects on health visits and outpatient use of children aged six to 15 . Compared with children without previous exposure to FHI, those with at least one year of treatment were more likely to visit health facilities by 2.3 percentage points. Similarly, they also had 0.092 more visits for outpatient care, but no significant difference was found for inpatient services. I find no evidence of the spill over effects of the policy on health utilization among these children. My results are consistent with recent evidence on the impact of subsidised health insurance on utilization in developing countries (Guindon, 2014; D. Levine et al., 2016; G. Miller et al., 2013; C. Nguyen, 2016; H. Nguyen \& Wang, 2013; M. Palmer et al., 2014; Panpiemras, Puttitanun, Samphantharak, \& Thampanishvong, 2011; Robyn et al., 2012; Sood et al., 2014).

There may be two reasons for the lack of impact on inpatient care. First, hospitalization can be less critical for older children, who are not as vulnerable to illness as young children. Second, the increase in outpatient care at primary facilities might provide effective treatment for these children so that they do not need tertiary care. Indeed, if primary facilities can produce good quality care and absorb much of the healthcare need, this can ease the increasing pressure on Vietnam's central hospitals.

It is noteworthy that previous studies did not take into account the persistent and spill over effects of $\mathrm{FHI}$ for young children when investigating the impact of $\mathrm{VHI}$ for school children. Since these studies often compared schoolchildren with and without insurance, they overlooked the potential impact of $\mathrm{FHI}$ in the past and the presence of younger siblings in households. Thus, they failed to control for the additional effects and might overstate the true impact of VHI for schoolchildren. 
Healthcare expenditure. At the individual level, I find that the FHI policy significantly increases spending on young children by 34 percent; outpatient care accounts for most of this increase in expenditure. My results also show that past exposure to $\mathrm{FHI}$ at a young age increases individual health spending by 13 percent and an additional year of $\mathrm{FHI}$ can further increase the expense by 3.6 percent. I find no evidence of the spill over impact of the FHI policy on healthcare expenditure among untargeted children. At the household level, I find no evidence of the policy's effects on household healthcare expenditure and the probability of incurring catastrophic healthcare costs. These results indicate that more healthcare utilization requires more payments; the FHI policy is unable to achieve its second goal to reduce healthcare expenditure.

The lack of financial protection by health insurance policies in Vietnam has been addressed in previous studies (C. Nguyen, 2016; H. Nguyen \& Wang, 2013; M. Palmer et al., 2014; Sepehri, Sarma, \& Oguzoglu, 2011). Van Minh et al. (2013) reported that health insurance in Vietnam covered 80 percent of health expenditure and health users paid for the remaining 20 percent. According to the Ministry of Health of Vietnam (2010), health insurance funds paid for 80 to 100 percent of health expenditure for insured at registered health facilities. However, in 2014, about 10 percent of the population spent over 10 percent of household income on healthcare. In 2012, two to three percent of the population was pushed into extreme poverty through medical expenses. Although the country started SHI in 1992, out-of-pocket spending in 2016 was still around 40 percent of total national health expenditure.

The lack of financial protection from SHI in Vietnam can be the result of inconsistencies in healthcare policies. On the one hand, the Vietnamese government gives people the incentive to use more healthcare but heavily regulates the salary of health workers. This can discourage people from pursuing a medical career (which is very time consuming) because they cannot charge patients accordingly. Matsushima, Yamada, and Shimamura (2020) showed that the number of doctors increased slowly during the coverage expansion. The shortage of health workers can negatively affect the quality of care and put even more constraints on the healthcare system.

On the other hand, under Decision No. 45/HDBT in 1989, the government allowed public health facilities to collect fees for care services. This has been considered one factor that 
increases out-of-pocket expenditure. Under the Health Insurance Law 2008, the insured must co-pay fees if at higher level of public health facilities (see in the healthcare system in the Introduction). In addition, Decree 43/ND-CP in 2006 encouraged hospitals to generate more income to pay for staff, as well as to invest in new technology and equipment. Consequently, Van Minh et al. (2013) argued that new technology would further increase healthcare costs but the hospital financial autonomy has transferred the financial burden to households. Thus, these policies and the prevalence of private clinics run by doctors (who work after hours) can increase the earnings of health workers but create greater costs for patients. In a field experiment conducted in China, Lu (2014) found that doctors who could earn a proportion of patients' health expenditure prescribed more expensive drugs for insured patients than uninsured.

This also creates another problem. (Vujicic, Shengelia, Alfano, \& Thu, 2011) shows that the shortage of doctors in rural areas has worsened because there are fewer earning options in the rural economy. This can explain my results that show higher health insurance rates of children in rural areas did not have a greater impact on their healthcare utilization compared with urban children.

Generally, Vietnamese patients can seek treatment for minor illness by visiting private clinics or buying over-the-counter drugs that is costly but more convenient. Otherwise, they can have more affordable care by visiting public hospitals which is crowded and time consuming. As Vietnamese tend to avoid hospital but prefer seeing private doctors and buying drug without prescription, they need to pay more healthcare even with health insurance. Additionally, under-table payment (a bribe) to health workers, a common practice among care seekers in Vietnam (Matsushima \& Yamada, 2016), and non-medical costs for accommodation, transportation and food can further increase out-of-pocket spending (M. G. Palmer, 2014; A. Wagstaff \& Doorslaer, 2003). These expenses can be devastating for poor patients from rural areas who can need inpatient care at higher-level health facilities.

Health status. In the attempt to examine whether coverage expansion can improve the health outcomes of Vietnamese children, I use information on their self-reporting of experiencing illness. The results show that the FHI policy appears to have no impact on the self-reported morbidity rates of young and older children. This finding is different from recent studies that 
show health insurance significantly improved self-reported physical and mental health conditions (Camacho \& Conover, 2013; Chen \& Jin, 2012; Finkelstein et al., 2012). A. Wagstaff and Doorslaer (2003) found that SHI in Vietnam has a positive impact on health outcomes. However, the author used different health indicators (height- and weight-for-age) and an older version of VHLSS conducted in 1993-1998.

Education outcomes. I find that the policy change can affect the educational outcomes of children aged six to 15 . Compared with children without $\mathrm{FHI}$ at a young age, one more year of exposure to the policy is associated with a 0.23 percentage point increase in school attendance. Consistent with previous evidence of positive effects of health insurance on children's school enrolment (Chen \& Jin, 2012), this study shows that living with children under six can increase the probability of attending school in the last 12 months by 2.8 percentage points. On the other hand, the results show that children with at least one year of free health care at a young age might receive higher education expenditure by 3.8 percent compared with the control group. In contrast, I find that the presence of young children in the household has no beneficial effect on education spending on older siblings. At the household level, I find that education expenditure per capita of a household with children under six is significantly lower than for households without young children.

Household outcomes. I find that providing FHI for young children can affect household outcomes. First, my estimates show that households of young children have higher wages than the control group by 9.8 percent. This is somewhat inconsistent with recent evidence that shows health insurance for the poor in Vietnam decreased the number of working hours as well as the probability of employment (N. Le et al., 2019). Though I find no significant effects of the policy on household income per capita, the results indicate that households with young children have lower expenditure per capita and adult equivalents than the control group by 3.8 and 2.4 percent, respectively.

My results also show that the policy has negligible effect on household caloric consumption. This finding is different from recent studies that show health insurance significantly improved self-reported physical and mental health conditions (Camacho \& Conover, 2013; Chen \& Jin, 2012; Finkelstein et al., 2012). While Peng and Conley (2016) reported that health insurance in rural China improved child nutrition status, I find that free health insurance policy might 
have negative effects on household calorie consumption. Though the estimates are statistically significant, the effect sizes are insignificant compared to the baseline. It should be noted that as I measure calories consumption per adult equivalent, this finding might be an inadequately informative indicator for children's health status.

\subsection{Contributions of the Study}

\subsubsection{Contribution to existing literature}

The evaluation of health insurance policy in Vietnam can provide empirical evidence to support policy decisions as well as inform budget planning. Although SHI has been promoted as an effective tool to achieve universal health coverage in Vietnam, recent studies on its effects provided mixed results of its impact (H. L. Dao, Somanathan, Tandon, Hurt, \& Fuenzalida-Puelma, 2014; Guindon, 2014; C. Nguyen, 2016; H. Nguyen \& Wang, 2013; M. Palmer et al., 2014). More importantly, these studies examine a small set of possible outcomes. In contrast, my study examines a wide range of possible impacts and is thus able to provide a more comprehensive perspective on the overall impact of the policy intervention.

A health insurance policy, and in fact any policy intervention, might not have immediate effects; the effects might appear over time (Ursula Giedion et al., 2013). As such, the period of time over which impacts are measured matters. Compared with previous studies, I examine the effects of health insurance over a more extended period. Using eight rounds of national household surveys, I investigate the effects of insurance policy 10 years after it was implemented. Using a more extensive dataset has the advantage of addressing the potential lag in FHI policy impact. For example, results discussed in Section 5.1.2.1 showed that the FHI policy for young children has greater effects on healthcare visits after ten years of implementation. Additionally, this dataset can capture the remarkable development in Vietnam in the last two decades.

Another problem arises when comparing the outcomes of insured populations before and after a policy change. It is challenging to find a plausible control group to account for changes in outcomes when the policy intervention is national in scope, and when there has been sustained development which is likely to improve health and other outcomes on its own. For 
example, using older children as a control group to study the impact of free health insurance effects (on health outcomes) is questionable if the two groups of children have different healthcare needs through differences in health condition. Most studies in Vietnam implicitly assume that children aged under 11 have comparable health status. I, on the other hand, explicitly use data on self-reported morbidity status to justify my choice of the control group. Specifically, I study the direct effects of health insurance on children under six using a control group of children aged six to ten. Since my data show that these two groups have similar patterns of self-reported morbidity, I argue that children aged six to ten can serve as a plausible counterfactual.

I also use exogenous variation in the timing of the policy change to study the persistent effects of health insurance across children in different age cohorts. More importantly, I also investigate the spill over effects of health insurance on other household members. I exploit the exogenous variations in the presence of children under six in households to compare older children with and without young siblings, households with and without young children.

To the best of my knowledge, this study is the first to examine the prolonged and spill over effects of health insurance on school-aged children and their households in Vietnam. Indeed, this study provides empirical evidence that can inform policymakers with a broad picture of the impact of the FHI policy for young children in Vietnam. As the country aims to achieve universal health coverage by 2030 , the government has considered increasing coverage to other populations such as the elderly. The findings of this study can be beneficial to that work.

\subsubsection{Policy Implications}

The significant increase in health insurance rates reported in this thesis can be attributed to some features of the design of Vietnam's existing social health insurance programme. First, the current participants who join social health insurance in the country are issued a universal health insurance card regardless of their socio-economic background. Before the issue of this single card, separate cards were given to different groups of participants: those who work in formal or private sectors, students, and those who were poor. This practice of labelling was likely to cause stigma to vulnerable groups such as those in poverty which, in turn, might lead them to avoiding enrolling their children in the programme. Also, those in poverty who used 
their insurance card might face discrimination at health facilities. Therefore, the use of a single health insurance card for all may go some way to eliminating the stigma and contributed to the increase in the reported health insurance uptake among young children in this study. Countries moving towards publically-funded universal health insurance should consider this practice when designing their health insurance schemes.

Second, even though the Vietnamese government subsidizes certain groups of the population at different rates for social health insurance, the premium and the benefit package is the same across these subgroups. Compared to the social health insurance in China whose benefit packages vary among different insurance plans with different premiums, the one-fits-all solution in Vietnam has the advantage of encouraging people to join the scheme. This feature, however, can limit the options of those who were willing to pay more for a higher level of care they expected. This can prompt them to opt for another private health insurance scheme which is more expensive and expected to provide more benefits than social health insurance. Indeed, since public hospitals, especially in big cities, are infamous for being overcrowded and lacking staffs, rooms, and equipment, wealthier individuals are more likely to join an insurance plan (if there is one) that charges higher premium for better care. In this case, this shows that Vietnamese government might try to avoid the inequality in healthcare due to income levels. Therefore, it is up to other governments to choose an appropriate model that works best for their people.

Third, social health insurance in Vietnam relies on gatekeepers to discourage people from bypassing health facilities at perceived lower levels of care such as commune health station and district hospitals. This restriction is useful for controlling health care costs and preventing central hospitals from being overloaded. However, since facilities of lower care quality are well-known for the lack of experienced doctors and essential equipment, the presence of gatekeepers may force current participants to bypass the system, thereby increasing their personal healthcare expenditure through self-funding their care. Moreover, it is a misnomer that gatekeeper alone can reduce the cost of care and fix the overcrowded central hospitals in Vietnam. Indeed, this study shows that despite the improvement in health insurance status, health expenditures have been gradually increasing in the country the last ten years. Consider a family from a small town that has to bypass the poor-condition district hospital to seek care at central hospital. Without referral from local hospitals (which are not easy to get), this family 
needs to bear the additional cost of foregone paycheck, transportation and accommodation fees which are significantly high, especially for low-income individuals. To fix this problem, the first task is for more government investment in human capital and equipment for local health facilities, This, however, is no easy task, especially given that current and historical national debt levels. Moreover, the economy is tightening still in response to the Covid-19 pandemic. A second fix would involve the referral procedure, which needs to be improved so that it is more accessible to insured individuals, especially those in rural areas who often lack of knowledge or awareness of the benefit packages and struggle with complicated paperwork and the administrative burden of using the programme. Third, I recommend that financial assistance for transportation and accommodation be included in the benefit packages for low-income individuals to help reduce the costs to accessing healthcare services.

Finally, the findings that there are significant spillover effects of providing free health insurance for young children has implications for policy. Many countries that have implemented universal health insurance programmes have not witnessed a significant increase in coverage in a short period of time, like in Vietnam and China. Empirical papers often attribute this achievement to the aggressive policy implementation and awareness campaigns in Vietnam and China. While other Asian populations are similar to Vietnam and China in that there is more intergenerational shared households and dense communities, it appears that awareness campaigns are an additional and important source for distributing knowledge.

\subsection{The Study's Limitations}

As studies using Difference-in-Differences models need to rely on the parallel trend assumption, it would have been better if I could have used more rounds of data to examine the trends in outcomes that happened before the policy change. Though VHLSS started in 2002, there were two rounds of its predecessor, the Living Standard Surveys in 1993 and 1998. These previous surveys did not provide consistent questionnaires whose data could be extracted. Even the 2002 survey, for instance, did not provide information on individual health insurance status and the number of outpatient and inpatient visits. Therefore, I interpret estimates on health insurance status and healthcare utilization with some caution. Likewise, since questions about self-reported morbidity were not included in 2002 and have 
changed since 2010, my DD models on morbidity rate relied on data restricted to three rounds of the surveys from 2004 to 2008. Besides, the design of VHLSSs did not allow me to identify the kin relationships in extended households. Therefore, I am unable to explore whether this policy change can affect parents' outcomes. Since 2014, it has been possible to match mothers with children. I look forward to seeing more detailed research on the effects on health insurance in Vietnam.

Moreover, the main source of data is from the VHLSS, a national household survey that has primarily focused on living standards. Thus, the information on healthcare and health behaviours are limited. More qualitative research in the future can contribute to a deeper understanding of how people actually think about health insurance, why ten percent of Vietnamese population are still uninsured, and what aspects of the current insurance programme need to be improved so that they can reduce the personal and national cost and bring more benefits to users.

\subsection{Conclusions}

Social Health Insurance started in Vietnam in 1992 to mobilize resources for a healthcare system that has been mainly financed by tax revenue and private spending. Vietnam is a developing country in South-East Asia where the informal sector is large, and the tax system is far from perfect. Thus, Vietnam is unable to collect enough tax revenue for healthcare which leads to the skyrocketing out-of-pocket expenditure that makes SHI seem desirable. Although $\mathrm{SHI}$ in the country has succeeded in expanding coverage of the population, private costs for healthcare are still high after many years of implementation.

To conclude, this thesis finds that the free health insurance policy for young children has significant impacts on the health outcomes of children under six, who are the policy's direct beneficiaries. I also find evidence of persistent effects of this policy on the health and educational outcomes of ex-beneficiaries. The policy has positive spill over effects on older (untargeted) children living in targeted households, positive effects on household wage income, income per capita, education expenditure, and insurance coverage but no impact on standard of living, health expenditure, the likelihood of catastrophic healthcare spending, or calorie consumption. 


\section{TABLES}

The regression results reported in the following tables are adjusted for year fixed effects. I also include control variables for the characteristics of children (age, gender, ethnicity) and of the household head (age, gender, education level). I also control for household characteristics such as rural/urban and regions; ${ }^{*}=p<0.05 ;{ }^{* *}=p<0.01$; and ${ }^{* * *}=p<0.001$. Standard errors are in parentheses. The tables with full details are presented in the Appendix. 
Table 5. 1 Direct Health Effect - Health Insurance Status (Children Aged 0-10)

\begin{tabular}{|c|c|c|c|c|}
\hline & $(1)$ & $(2)$ & (3) & (4) \\
\hline \multirow[t]{2}{*}{ Under Six } & $-0.300 * * *$ & $-0.300 * * *$ & $-0.301 * * *$ & $-0.300 * * *$ \\
\hline & $(0.010)$ & $(0.011)$ & $(0.010)$ & $(0.014)$ \\
\hline \multirow[t]{2}{*}{ Under Six $x$ Post } & $0.410 * * *$ & & $0.383 * * *$ & $0.413 * * *$ \\
\hline & $(0.013)$ & & $(0.01)$ & $(0.02)$ \\
\hline \multirow[t]{2}{*}{ Under Six x Year2006 } & & $0.440 * * *$ & & \\
\hline & & $(0.010)$ & & \\
\hline \multirow[t]{2}{*}{ Under Six x Year2008 } & & $0.431^{* * *}$ & & \\
\hline & & $(0.010)$ & & \\
\hline \multirow[t]{2}{*}{ Under Six x Year2010 } & & $0.450 * * *$ & & \\
\hline & & $(0.010)$ & & \\
\hline \multirow[t]{2}{*}{ Under Six x Year2012 } & & $0.411^{* * *}$ & & \\
\hline & & $(0.012)$ & & \\
\hline \multirow[t]{2}{*}{ Under Six x Year2014 } & & $0.380 * * *$ & & \\
\hline & & $(0.011)$ & & \\
\hline \multirow[t]{2}{*}{ Under Six x Year2016 } & & $0.381 * * *$ & & \\
\hline & & $(0.013)$ & & \\
\hline \multirow[t]{2}{*}{ Under Six x Post $x$ Rural } & & & $0.042 * * *$ & \\
\hline & & & $(0.007)$ & \\
\hline \multirow[t]{2}{*}{ Under Six x Post x Region 1} & & & & $0.025^{*}$ \\
\hline & & & & $(0.010)$ \\
\hline \multirow[t]{2}{*}{ Under Six x Post x Region 2} & & & & $-0.040 * * *$ \\
\hline & & & & $(0.014)$ \\
\hline \multirow[t]{2}{*}{ Under Six x Post $x$ Region 3} & & & & 0.015 \\
\hline & & & & $(0.011)$ \\
\hline \multirow[t]{2}{*}{ Under Six $x$ Post $x$ Region 5} & & & & -0.023 \\
\hline & & & & $(0.010)$ \\
\hline \multirow[t]{2}{*}{ Under Six x Post $\mathrm{x}$ Region 6} & & & & $0.032^{*}$ \\
\hline & & & & $(0.011)$ \\
\hline \multirow[t]{2}{*}{ Constant } & $0.501 * * *$ & $0.501 * * *$ & $0.520 * * *$ & $0.500 * * *$ \\
\hline & $(0.021)$ & $(0.020)$ & $(0.020)$ & $(0.033)$ \\
\hline Obs & 44520 & 44520 & 44520 & 44520 \\
\hline R Squared & 0.254 & 0.255 & 0.254 & 0.255 \\
\hline
\end{tabular}


Table 5. 2 Direct Health Effect - Health Insurance Status (Children Aged 2-10)

\begin{tabular}{|c|c|c|c|c|}
\hline & (1) & (2) & (3) & (4) \\
\hline Under Six & $\begin{array}{r}-0.311^{* * *} \\
(0.010)\end{array}$ & $\begin{array}{r}-0.311 * * * \\
(0.010)\end{array}$ & $\begin{array}{r}-0.310 * * * \\
(0.012)\end{array}$ & $\begin{array}{r}-0.310 * * * \\
(0.014)\end{array}$ \\
\hline Under Six x Post & $\begin{array}{r}0.403 * * * \\
(0.010)\end{array}$ & & $\begin{array}{r}0.360 * * * \\
(0.010)\end{array}$ & $\begin{array}{r}0.400 * * * \\
(0.020)\end{array}$ \\
\hline Under Six x Year2006 & & $\begin{array}{r}0.442^{* * *} \\
(0.020)\end{array}$ & & \\
\hline Under Six x Year2008 & & $\begin{array}{r}0.420 * * * \\
(0.020)\end{array}$ & & \\
\hline Under Six x Year2010 & & $\begin{array}{r}0.440 * * * \\
(0.011)\end{array}$ & & \\
\hline Under Six x Year2012 & & $\begin{array}{r}0.390 * * * \\
(0.010)\end{array}$ & & \\
\hline Under Six x Year2014 & & $\begin{array}{r}0.352^{* * *} \\
(0.013)\end{array}$ & & \\
\hline Under Six x Year2016 & & $\begin{array}{r}0.361^{* * *} \\
(0.010)\end{array}$ & & \\
\hline Under Six x Post $x$ Rural & & & $\begin{array}{r}0.048 * * * \\
(0.007)\end{array}$ & \\
\hline Under Six $x$ Post $x$ Region 1 & & & & $\begin{array}{r}0.010 \\
(0.014)\end{array}$ \\
\hline Under Six x Post $x$ Region 2 & & & & $\begin{array}{r}-0.029 * \\
(0.012)\end{array}$ \\
\hline Under Six x Post $x$ Region 3 & & & & $\begin{array}{l}0.0036 \\
(0.011)\end{array}$ \\
\hline Under Six x Post x Region 5 & & & & $\begin{array}{l}-0.029 \\
(0.020)\end{array}$ \\
\hline Under Six x Post $x$ Region 6 & & & & $\begin{array}{l}0.030 * \\
(0.010)\end{array}$ \\
\hline Constant & $\begin{array}{r}0.591 * * * \\
(0.032)\end{array}$ & $\begin{array}{r}0.591 * * * \\
(0.030)\end{array}$ & $\begin{array}{r}0.610 * * * \\
(0.030)\end{array}$ & $\begin{array}{r}0.590 * * * \\
(0.031)\end{array}$ \\
\hline Obs & 37217 & 37217 & 37217 & 37217 \\
\hline R Squared & 0.226 & 0.228 & 0.227 & 0.227 \\
\hline
\end{tabular}


Table 5. 3 Direct Health Effect - Health Insurance Status (Children Aged 4-7)

\begin{tabular}{|c|c|c|c|c|}
\hline & $(1)$ & $(2)$ & (3) & (4) \\
\hline Under Six & $\begin{array}{r}-0.201 * * * \\
(0.021)\end{array}$ & $\begin{array}{r}-0.201^{* * *} \\
(0.020)\end{array}$ & $\begin{array}{r}-0.203^{* * *} \\
(0.022)\end{array}$ & $\begin{array}{r}-0.200 * * * \\
(0.020)\end{array}$ \\
\hline Under Six x Post & $\begin{array}{r}0.311^{* * *} \\
(0.020)\end{array}$ & & $\begin{array}{r}0.290 * * * \\
(0.021)\end{array}$ & $\begin{array}{r}0.320 * * * \\
(0.031)\end{array}$ \\
\hline Under Six x Year2006 & & $\begin{array}{r}0.360 * * * \\
(0.021)\end{array}$ & & \\
\hline Under Six x Year2008 & & $\begin{array}{r}0.330 * * * \\
(0.020)\end{array}$ & & \\
\hline Under Six x Year2010 & & $\begin{array}{r}0.360 * * * \\
(0.021)\end{array}$ & & \\
\hline Under Six x Year2012 & & $\begin{array}{r}0.290 * * * \\
(0.020)\end{array}$ & & \\
\hline Under Six x Year2014 & & $\begin{array}{r}0.261^{* * *} \\
(0.021)\end{array}$ & & \\
\hline Under Six x Year2016 & & $\begin{array}{r}0.260 * * * \\
(0.020)\end{array}$ & & \\
\hline Under Six x Post $x$ Rural & & & $\begin{array}{r}0.037^{* * *} \\
(0.010)\end{array}$ & \\
\hline Under Six x Post x Region 1 & & & & $\begin{array}{r}0.003 \\
(0.020)\end{array}$ \\
\hline Under Six x Post x Region 2 & & & & $\begin{array}{c}-0.044^{*} \\
(0.020)\end{array}$ \\
\hline Under Six x Post x Region 3 & & & & $\begin{array}{r}0.002 \\
(0.021)\end{array}$ \\
\hline Under Six x Post x Region 5 & & & & $\begin{array}{c}-0.058^{*} \\
(0.022)\end{array}$ \\
\hline Under Six x Post x Region 6 & & & & $\begin{array}{r}0.025 \\
(0.020)\end{array}$ \\
\hline Constant & $\begin{array}{r}0.782^{* * *} \\
(0.081)\end{array}$ & $\begin{array}{r}0.791 * * * \\
(0.081)\end{array}$ & $\begin{array}{r}0.792^{* * *} \\
(0.080)\end{array}$ & $\begin{array}{r}0.784 * * * \\
(0.080)\end{array}$ \\
\hline Obs & 16,075 & 16,075 & 16,075 & 16,075 \\
\hline R Squared & 0.251 & 0.254 & 0.251 & 0.252 \\
\hline
\end{tabular}


Table 5. 4 Direct Health Effect - Health Visit (Children Aged 0-10)

\begin{tabular}{|c|c|c|c|c|}
\hline & (1) & (2) & (3) & (4) \\
\hline \multirow[t]{2}{*}{ Under Six } & -0.009 & -0.011 & -0.009 & -0.009 \\
\hline & (0.010) & $(0.011)$ & (0.010) & (0.010) \\
\hline \multirow[t]{2}{*}{ Under Six x Post } & $0.081 * * *$ & & $0.12 * * *$ & $0.088 * * *$ \\
\hline & $(0.012)$ & & $(0.014)$ & $(0.020)$ \\
\hline \multirow[t]{2}{*}{ Under Six x Year2006 } & & $0.029 *$ & & \\
\hline & & $(0.020)$ & & \\
\hline \multirow[t]{2}{*}{ Under Six x Year2008 } & & 0.018 & & \\
\hline & & $(0.020)$ & & \\
\hline \multirow[t]{2}{*}{ Under Six x Year2010 } & & $0.059 * * *$ & & \\
\hline & & $(0.021)$ & & \\
\hline \multirow[t]{2}{*}{ Under Six x Year2012 } & & $0.090 * * *$ & & \\
\hline & & $(0.023)$ & & \\
\hline \multirow[t]{2}{*}{ Under Six x Year2014 } & & $0.160 * * *$ & & \\
\hline & & $(0.020)$ & & \\
\hline \multirow[t]{2}{*}{ Under Six x Year2016 } & & $0.131 * * *$ & & \\
\hline & & $(0.024)$ & & \\
\hline \multirow[t]{2}{*}{ Under Six $x$ Post $x$ Rural } & & & $-0.049 * * *$ & \\
\hline & & & $(0.010)$ & \\
\hline \multirow[t]{2}{*}{ Under Six x Post $x$ Region 1} & & & & 0.030 \\
\hline & & & & $(0.021)$ \\
\hline \multirow[t]{2}{*}{ Under Six x Post $x$ Region 2} & & & & -0.010 \\
\hline & & & & $(0.021)$ \\
\hline \multirow[t]{2}{*}{ Under Six x Post x Region 3} & & & & -0.022 \\
\hline & & & & $(0.020)$ \\
\hline \multirow[t]{2}{*}{ Under Six x Post $x$ Region 5} & & & & -0.008 \\
\hline & & & & $(0.020)$ \\
\hline \multirow[t]{2}{*}{ Under Six x Post $x$ Region 6} & & & & -0.027 \\
\hline & & & & $(0.021)$ \\
\hline \multirow[t]{2}{*}{ Constant } & $0.731 * * *$ & $0.731 * * *$ & $0.715^{* * *}$ & $0.720 * * *$ \\
\hline & $(0.040)$ & $(0.040)$ & $(0.041)$ & (0.041) \\
\hline Obs & 44,520 & 44,520 & 44,520 & 44,520 \\
\hline R Squared & 0.086 & 0.088 & 0.087 & 0.087 \\
\hline
\end{tabular}


Table 5. 5 Direct Health Effect - Health Visit (Children Aged 2-10)

\begin{tabular}{|c|c|c|c|c|}
\hline & (1) & $(2)$ & (3) & (4) \\
\hline Under Six & $\begin{array}{r}-0.043^{* * *} \\
(0.020)\end{array}$ & $\begin{array}{r}-0.043^{* * *} \\
(0.020)\end{array}$ & $\begin{array}{r}-0.043 * * * \\
(0.021)\end{array}$ & $\begin{array}{r}-0.043^{* * *} \\
(0.020)\end{array}$ \\
\hline Under Six x Post & $\begin{array}{r}0.067^{* * *} \\
(0.013)\end{array}$ & & $\begin{array}{r}0.11 * * * \\
(0.020)\end{array}$ & $\begin{array}{r}0.080^{* * *} \\
(0.020)\end{array}$ \\
\hline Under Six x Year2006 & & $\begin{array}{r}0.027 \\
(0.021)\end{array}$ & & \\
\hline Under Six x Year2008 & & $\begin{array}{r}0.013 \\
(0.021)\end{array}$ & & \\
\hline Under Six x Year2010 & & $\begin{array}{r}0.055^{* * *} \\
(0.022)\end{array}$ & & \\
\hline Under Six x Year2012 & & $\begin{array}{r}0.073 * * * \\
(0.021)\end{array}$ & & \\
\hline Under Six x Year2014 & & $\begin{array}{r}0.120 * * * \\
(0.024)\end{array}$ & & \\
\hline Under Six x Year2016 & & $\begin{array}{r}0.101^{* * *} \\
(0.023)\end{array}$ & & \\
\hline Under Six x Post $x$ Rural & & & $\begin{array}{r}-0.056 * * * \\
(0.012)\end{array}$ & \\
\hline Under Six x Post x Region 1 & & & & $\begin{array}{r}0.012 \\
(0.022)\end{array}$ \\
\hline Under Six x Post x Region 2 & & & & $\begin{array}{l}-0.025 \\
(0.020)\end{array}$ \\
\hline Under Six x Post $x$ Region 3 & & & & $\begin{array}{l}-0.030 \\
(0.020)\end{array}$ \\
\hline Under Six x Post $x$ Region 5 & & & & $\begin{array}{r}-0.0077 \\
(0.020)\end{array}$ \\
\hline Under Six x Post x Region 6 & & & & $\begin{array}{l}-0.014 \\
(0.020)\end{array}$ \\
\hline Constant & $\begin{array}{r}0.901 * * * \\
(0.040)\end{array}$ & $\begin{array}{r}0.912 * * * \\
(0.040)\end{array}$ & $\begin{array}{r}0.891^{* * *} \\
(0.041)\end{array}$ & $\begin{array}{r}0.900 * * * \\
(0.041)\end{array}$ \\
\hline Obs & 37,217 & 37,217 & 37,217 & 37,217 \\
\hline R Squared & 0.081 & 0.083 & 0.082 & 0.082 \\
\hline
\end{tabular}


Table 5. 6 Direct Health Effect - Health Visit (Children Aged 4-7)

\begin{tabular}{|c|c|c|c|c|}
\hline & (1) & $(2)$ & (3) & (4) \\
\hline \multirow[t]{2}{*}{ Under Six } & 0.001 & 0.001 & 0.001 & 0.001 \\
\hline & $(0.030)$ & $(0.030)$ & $(0.030)$ & $(0.030)$ \\
\hline \multirow[t]{2}{*}{ Under Six x Post } & 0.005 & & 0.014 & 0.004 \\
\hline & $(0.021)$ & & $(0.022)$ & (0.033) \\
\hline \multirow[t]{2}{*}{ Under Six x Year2006 } & & -0.008 & & \\
\hline & & $(0.031)$ & & \\
\hline \multirow[t]{2}{*}{ Under Six x Year2008 } & & -0.041 & & \\
\hline & & $(0.030)$ & & \\
\hline \multirow[t]{2}{*}{ Under Six x Year2010 } & & 0.033 & & \\
\hline & & $(0.030)$ & & \\
\hline \multirow[t]{2}{*}{ Under Six x Year2012 } & & -0.004 & & \\
\hline & & $(0.032)$ & & \\
\hline \multirow[t]{2}{*}{ Under Six x Year2014 } & & 0.034 & & \\
\hline & & $(0.032)$ & & \\
\hline \multirow[t]{2}{*}{ Under Six x Year2016 } & & 0.009 & & \\
\hline & & $(0.030)$ & & \\
\hline \multirow[t]{2}{*}{ Under Six x Post $x$ Rural } & & & -0.011 & \\
\hline & & & $(0.020)$ & \\
\hline \multirow[t]{2}{*}{ Under Six x Post $x$ Region 1} & & & & 0.016 \\
\hline & & & & $(0.030)$ \\
\hline \multirow[t]{2}{*}{ Under Six $x$ Post $x$ Region 2} & & & & -0.005 \\
\hline & & & & $(0.033)$ \\
\hline \multirow[t]{2}{*}{ Under Six x Post $x$ Region 3} & & & & -0.008 \\
\hline & & & & $(0.031)$ \\
\hline \multirow[t]{2}{*}{ Under Six x Post $x$ Region 5} & & & & -0.003 \\
\hline & & & & $(0.040)$ \\
\hline \multirow[t]{2}{*}{ Under Six x Post $\times$ Region 6} & & & & 0.007 \\
\hline & & & & $(0.030)$ \\
\hline \multirow[t]{2}{*}{ Constant } & $0.701 * * *$ & $0.694 * * *$ & $0.694 * * *$ & $0.701 * * *$ \\
\hline & (0.102) & $(0.101)$ & $(0.100)$ & $(0.100)$ \\
\hline Obs & 16,075 & 16,075 & 16,075 & 16,075 \\
\hline R Squared & 0.060 & 0.061 & 0.060 & 0.061 \\
\hline
\end{tabular}


Table 5. 7 Direct Health Effect - The number of Outpatient Visit (Children Aged 0-10)

\begin{tabular}{|c|c|c|c|c|}
\hline & (1) & $(2)$ & (3) & (4) \\
\hline \multirow[t]{2}{*}{ Under Six } & 0.031 & 0.026 & 0.033 & 0.030 \\
\hline & (0.079) & (0.079) & $(0.074)$ & $(0.074)$ \\
\hline \multirow[t]{2}{*}{ Under Six x Post } & $0.291^{* * *}$ & & $0.580 * * *$ & $0.240 * *$ \\
\hline & $(0.061)$ & & (0.091) & $(0.100)$ \\
\hline \multirow[t]{2}{*}{ Under Six x Year2006 } & & 0.130 & & \\
\hline & & $(0.090)$ & & \\
\hline \multirow[t]{2}{*}{ Under Six x Year2008 } & & 0.071 & & \\
\hline & & $(0.080)$ & & \\
\hline \multirow[t]{2}{*}{ Under Six x Year2010 } & & $0.320 * * *$ & & \\
\hline & & $(0.100)$ & & \\
\hline \multirow[t]{2}{*}{ Under Six x Year2012 } & & $0.281 * * *$ & & \\
\hline & & $(0.080)$ & & \\
\hline \multirow[t]{2}{*}{ Under Six x Year2014 } & & $0.510 * * *$ & & \\
\hline & & $(0.081)$ & & \\
\hline \multirow{2}{*}{ Under Six x Year2016 } & & $0.401^{* * *}$ & & \\
\hline & & $(0.080)$ & & \\
\hline \multirow[t]{2}{*}{ Under Six x Post $x$ Rural } & & & $-0.402 * * *$ & \\
\hline & & & $(0.061)$ & \\
\hline \multirow[t]{2}{*}{ Under Six x Post x Region 1} & & & & 0.020 \\
\hline & & & & $(0.091)$ \\
\hline \multirow[t]{2}{*}{ Under Six x Post x Region 2} & & & & $-0.310 * * *$ \\
\hline & & & & $(0.080)$ \\
\hline \multirow[t]{2}{*}{ Under Six x Post $x$ Region 3} & & & & -0.082 \\
\hline & & & & $(0.091)$ \\
\hline \multirow[t]{2}{*}{ Under Six $x$ Post $x$ Region 5} & & & & $0.231^{*}$ \\
\hline & & & & $(0.100)$ \\
\hline \multirow[t]{2}{*}{ Under Six x Post $\times$ Region 6} & & & & $0.531 * * *$ \\
\hline & & & & $(0.100)$ \\
\hline \multirow[t]{2}{*}{ Constant } & $2.321 * * *$ & $2.330 * * *$ & $2.180 * * *$ & $2.380 * * *$ \\
\hline & $(0.210)$ & $(0.204)$ & $(0.200)$ & $(0.200)$ \\
\hline Obs & 44,520 & 44,520 & 44,520 & 44,520 \\
\hline R Squared & 0.095 & 0.096 & 0.096 & 0.098 \\
\hline
\end{tabular}


Table 5. 8 Direct Health Effect - The number of Outpatient Visit (Children Aged 2-10)

\begin{tabular}{|c|c|c|c|c|}
\hline & (1) & $(2)$ & (3) & (4) \\
\hline Under Six & $\begin{array}{r}-0.200 * * \\
(0.080)\end{array}$ & $\begin{array}{r}-0.200^{* *} \\
(0.080)\end{array}$ & $\begin{array}{r}-0.196 * * \\
(0.080)\end{array}$ & $\begin{array}{r}-0.200^{* *} \\
(0.080)\end{array}$ \\
\hline Under Six x Post & $\begin{array}{r}0.291^{* * *} \\
(0.070)\end{array}$ & & $\begin{array}{r}0.580^{* * *} \\
(0.091)\end{array}$ & $\begin{array}{r}0.210 * * \\
(0.102)\end{array}$ \\
\hline Under Six x Year2006 & & $\begin{array}{r}0.210^{* *} \\
(0.100)\end{array}$ & & \\
\hline Under Six x Year2008 & & $\begin{array}{r}0.029 \\
(0.090)\end{array}$ & & \\
\hline Under Six x Year2010 & & $\begin{array}{r}0.341 * * * \\
(0.101)\end{array}$ & & \\
\hline Under Six x Year2012 & & $\begin{array}{r}0.301 * * * \\
(0.090)\end{array}$ & & \\
\hline Under Six x Year2014 & & $\begin{array}{r}0.470 * * * \\
(0.091)\end{array}$ & & \\
\hline Under Six x Year2016 & & $\begin{array}{r}0.370 * * * \\
(0.090)\end{array}$ & & \\
\hline Under Six x Post $x$ Rural & & & $\begin{array}{r}-0.380 * * * \\
(0.070)\end{array}$ & \\
\hline Under Six x Post x Region 1 & & & & $\begin{array}{r}-0.033 \\
(0.100)\end{array}$ \\
\hline Under Six x Post x Region 2 & & & & $\begin{array}{r}-0.270 * * * \\
(0.090)\end{array}$ \\
\hline Under Six x Post $x$ Region 3 & & & & $\begin{array}{l}-0.055 \\
(0.100)\end{array}$ \\
\hline Under Six x Post x Region 5 & & & & $\begin{array}{c}0.310 * * \\
(0.101)\end{array}$ \\
\hline Under Six x Post x Region 6 & & & & $\begin{array}{r}0.620^{* * *} \\
(0.100)\end{array}$ \\
\hline Constant & $\begin{array}{r}3.120 * * * \\
(0.200)\end{array}$ & $\begin{array}{r}3.130 * * * \\
(0.200)\end{array}$ & $\begin{array}{r}3.010^{* * *} \\
(0.201)\end{array}$ & $\begin{array}{r}3.181 * * * \\
(0.201)\end{array}$ \\
\hline Obs & 37,217 & 37,217 & 37,217 & 37,217 \\
\hline R Squared & 0.094 & 0.094 & 0.095 & 0.097 \\
\hline
\end{tabular}


Table 5. 9 Direct Health Effect - The number of Outpatient Visit (Children Aged 4-7)

\begin{tabular}{|c|c|c|c|c|}
\hline & $(1)$ & $(2)$ & (3) & (4) \\
\hline Under Six & $\begin{array}{r}-0.031 \\
(0.101)\end{array}$ & $\begin{array}{r}-0.036 \\
(0.100)\end{array}$ & $\begin{array}{r}-0.030 \\
(0.100)\end{array}$ & $\begin{array}{c}-0.032 \\
(0.100)\end{array}$ \\
\hline Under Six x Post & $\begin{array}{r}0.072 \\
(0.090)\end{array}$ & & $\begin{array}{r}0.210 \\
(0.103)\end{array}$ & $\begin{array}{r}0.130 \\
(0.100)\end{array}$ \\
\hline Under Six x Year2006 & & $\begin{array}{r}0.078 \\
(0.100)\end{array}$ & & \\
\hline Under Six x Year2008 & & $\begin{array}{r}-0.170 \\
(0.100)\end{array}$ & & \\
\hline Under Six x Year2010 & & $\begin{array}{r}0.220 \\
(0.111)\end{array}$ & & \\
\hline Under Six x Year2012 & & $\begin{array}{r}0.081 \\
(0.100)\end{array}$ & & \\
\hline Under Six x Year2014 & & $\begin{array}{r}0.200 \\
(0.100)\end{array}$ & & \\
\hline Under Six x Year2016 & & $\begin{array}{r}-0.018 \\
(0.100)\end{array}$ & & \\
\hline Under Six x Post $x$ Rural & & & $\begin{array}{c}-0.180 * \\
(0.103)\end{array}$ & \\
\hline Under Six x Post x Region 1 & & & & $\begin{array}{l}-0.200 \\
(0.100)\end{array}$ \\
\hline Under Six x Post $x$ Region 2 & & & & $\begin{array}{r}-0.250 * \\
(0.100)\end{array}$ \\
\hline Under Six x Post $x$ Region 3 & & & & $\begin{array}{l}-0.063 \\
(0.100)\end{array}$ \\
\hline Under Six x Post x Region 5 & & & & $\begin{array}{c}-0.002 \\
(0.200)\end{array}$ \\
\hline Under Six x Post x Region 6 & & & & $\begin{array}{r}0.250 \\
(0.200)\end{array}$ \\
\hline Constant & $\begin{array}{r}1.750 * * \\
(0.700)\end{array}$ & $\begin{array}{r}1.770 * * \\
(0.700)\end{array}$ & $\begin{array}{r}1.691 * * \\
(0.702)\end{array}$ & $\begin{array}{r}1.730 * * \\
(0.701)\end{array}$ \\
\hline Obs & 16,075 & 16,075 & 16,075 & 16,075 \\
\hline R Squared & 0.082 & 0.082 & 0.082 & 0.083 \\
\hline
\end{tabular}


Table 5. 10 Direct Health Effect - The number of Inpatient Visit (Children Aged 0-10)

\begin{tabular}{|c|c|c|c|c|}
\hline & (1) & $(2)$ & (3) & $(4)$ \\
\hline \multirow[t]{2}{*}{ Under Six } & 0.002 & 0.002 & 0.002 & 0.003 \\
\hline & (0.010) & (0.010) & (0.010) & $(0.011)$ \\
\hline \multirow[t]{2}{*}{ Under Six $x$ Post } & $0.023 * *$ & & 0.021 & 0.012 \\
\hline & $(0.011)$ & & (0.010) & $(0.020)$ \\
\hline \multirow[t]{2}{*}{ Under Six x Year2006 } & & -0.003 & & \\
\hline & & $(0.010)$ & & \\
\hline \multirow[t]{2}{*}{ Under Six x Year2008 } & & $0.027^{*}$ & & \\
\hline & & (0.010) & & \\
\hline \multirow[t]{2}{*}{ Under Six x Year2010 } & & 0.009 & & \\
\hline & & $(0.020)$ & & \\
\hline \multirow[t]{2}{*}{ Under Six x Year2012 } & & $0.029 * *$ & & \\
\hline & & $(0.010)$ & & \\
\hline \multirow[t]{2}{*}{ Under Six x Year2014 } & & $0.050 * * *$ & & \\
\hline & & $(0.020)$ & & \\
\hline \multirow[t]{2}{*}{ Under Six x Year2016 } & & 0.024 & & \\
\hline & & $(0.020)$ & & \\
\hline \multirow[t]{2}{*}{ Under Six x Post $x$ Rural } & & & 0.002 & \\
\hline & & & (0.010) & \\
\hline \multirow[t]{2}{*}{ Under Six x Post x Region 1} & & & & $0.035 * *$ \\
\hline & & & & $(0.020)$ \\
\hline \multirow[t]{2}{*}{ Under Six x Post x Region 2} & & & & $0.031 *$ \\
\hline & & & & $(0.020)$ \\
\hline \multirow[t]{2}{*}{ Under Six x Post $x$ Region 3} & & & & 0.015 \\
\hline & & & & $(0.020)$ \\
\hline \multirow[t]{2}{*}{ Under Six $x$ Post $x$ Region 5} & & & & -0.022 \\
\hline & & & & $(0.020)$ \\
\hline \multirow[t]{2}{*}{ Under Six x Post x Region 6} & & & & -0.018 \\
\hline & & & & $(0.022)$ \\
\hline \multirow[t]{2}{*}{ Constant } & $0.100 * * *$ & $0.100 * * *$ & $0.100 * * *$ & $0.110 * * *$ \\
\hline & $(0.030)$ & $(0.030)$ & $(0.030)$ & $(0.030)$ \\
\hline Obs & 44,520 & 44,520 & 44,520 & 44,520 \\
\hline R Squared & 0.011 & 0.011 & 0.011 & 0.011 \\
\hline
\end{tabular}


Table 5. 11 Direct Health Effect - The number of Inpatient Visit (Children Aged 2-10)

\begin{tabular}{|c|c|c|c|c|}
\hline & (1) & $(2)$ & (3) & (4) \\
\hline \multirow[t]{2}{*}{ Under Six } & -0.021 & -0.021 & -0.021 & -0.021 \\
\hline & $(0.010)$ & (0.010) & (0.010) & $(0.010)$ \\
\hline \multirow[t]{2}{*}{ Under Six x Post } & 0.015 & & 0.012 & 0.013 \\
\hline & (0.010) & & $(0.010)$ & $(0.020)$ \\
\hline \multirow[t]{2}{*}{ Under Six x Year2006 } & & -0.008 & & \\
\hline & & $(0.020)$ & & \\
\hline \multirow[t]{2}{*}{ Under Six x Year2008 } & & 0.015 & & \\
\hline & & $(0.021)$ & & \\
\hline \multirow[t]{2}{*}{ Under Six x Year2010 } & & 0.016 & & \\
\hline & & $(0.021)$ & & \\
\hline \multirow[t]{2}{*}{ Under Six x Year2012 } & & 0.016 & & \\
\hline & & $(0.022)$ & & \\
\hline \multirow[t]{2}{*}{ Under Six x Year2014 } & & $0.039 * *$ & & \\
\hline & & $(0.020)$ & & \\
\hline \multirow[t]{2}{*}{ Under Six x Year2016 } & & 0.012 & & \\
\hline & & $(0.020)$ & & \\
\hline \multirow[t]{2}{*}{ Under Six x Post $x$ Rural } & & & 0.004 & \\
\hline & & & (0.011) & \\
\hline \multirow[t]{2}{*}{ Under Six x Post $x$ Region 1} & & & & 0.011 \\
\hline & & & & $(0.021)$ \\
\hline \multirow[t]{2}{*}{ Under Six $x$ Post $x$ Region 2} & & & & 0.014 \\
\hline & & & & $(0.020)$ \\
\hline \multirow[t]{2}{*}{ Under Six x Post $x$ Region 3} & & & & 0.007 \\
\hline & & & & $(0.020)$ \\
\hline \multirow[t]{2}{*}{ Under Six x Post $x$ Region 5} & & & & -0.020 \\
\hline & & & & $(0.022)$ \\
\hline \multirow[t]{2}{*}{ Under Six x Post $\times$ Region 6} & & & & -0.014 \\
\hline & & & & $(0.022)$ \\
\hline \multirow[t]{2}{*}{ Constant } & $0.200 * * *$ & $0.200 * * *$ & $0.210 * * *$ & $0.200 * * *$ \\
\hline & $(0.041)$ & (0.040) & $(0.040)$ & $(0.040)$ \\
\hline Obs & 37,217 & 37,217 & 37,217 & 37,217 \\
\hline R Squared & 0.010 & 0.010 & 0.010 & 0.010 \\
\hline
\end{tabular}


Table 5. 12 Direct Health Effect - The number of Inpatient Visit (Children Aged 4-7)

\begin{tabular}{|c|c|c|c|c|}
\hline & (1) & $(2)$ & (3) & (4) \\
\hline \multirow[t]{2}{*}{ Under Six } & 0.015 & 0.015 & 0.015 & 0.015 \\
\hline & (0.020) & $(0.020)$ & $(0.020)$ & $(0.020)$ \\
\hline \multirow[t]{2}{*}{ Under Six x Post } & 0.004 & & 0.000 & 0.009 \\
\hline & $(0.021)$ & & $(0.021)$ & $(0.020)$ \\
\hline \multirow[t]{2}{*}{ Under Six x Year2006 } & & -0.018 & & \\
\hline & & $(0.020)$ & & \\
\hline \multirow[t]{2}{*}{ Under Six x Year2008 } & & 0.000 & & \\
\hline & & $(0.020)$ & & \\
\hline \multirow[t]{2}{*}{ Under Six x Year2010 } & & 0.016 & & \\
\hline & & $(0.020)$ & & \\
\hline \multirow[t]{2}{*}{ Under Six x Year2012 } & & 0.005 & & \\
\hline & & $(0.020)$ & & \\
\hline \multirow[t]{2}{*}{ Under Six x Year2014 } & & 0.033 & & \\
\hline & & $(0.020)$ & & \\
\hline \multirow[t]{2}{*}{ Under Six x Year2016 } & & -0.017 & & \\
\hline & & $(0.020)$ & & \\
\hline \multirow[t]{2}{*}{ Under Six x Post $x$ Rural } & & & 0.005 & \\
\hline & & & (0.011) & \\
\hline \multirow[t]{2}{*}{ Under Six x Post x Region 1} & & & & 0.007 \\
\hline & & & & $(0.020)$ \\
\hline \multirow[t]{2}{*}{ Under Six x Post x Region 2} & & & & -0.006 \\
\hline & & & & $(0.030)$ \\
\hline \multirow[t]{2}{*}{ Under Six x Post x Region 3} & & & & -0.017 \\
\hline & & & & $(0.020)$ \\
\hline \multirow[t]{2}{*}{ Under Six x Post $x$ Region 5} & & & & -0.013 \\
\hline & & & & $(0.030)$ \\
\hline \multirow[t]{2}{*}{ Under Six x Post x Region 6} & & & & -0.000 \\
\hline & & & & $(0.030)$ \\
\hline \multirow[t]{2}{*}{ Constant } & $0.201 * *$ & $0.201 * *$ & $0.201 * *$ & $0.200 * *$ \\
\hline & $(0.100)$ & $(0.100)$ & $(0.102)$ & $(0.101)$ \\
\hline Obs & 16,075 & 16,075 & 16,075 & 16,075 \\
\hline R Squared & 0.007 & 0.008 & 0.007 & 0.007 \\
\hline
\end{tabular}


Table 5. 13 Direct Health Effect - Individual Health Expenditure (Children Aged 0-10)

\begin{tabular}{|c|c|c|c|c|}
\hline & (1) & (2) & (3) & (4) \\
\hline \multirow[t]{2}{*}{ Under Six } & $0.082 * *$ & 0.022 & $0.081 * *$ & $0.080 * *$ \\
\hline & $(0.041)$ & $(0.041)$ & $(0.040)$ & $(0.040)$ \\
\hline \multirow[t]{2}{*}{ Under Six x Post } & $0.340 * * *$ & & $0.700 * * *$ & $0.220 * * *$ \\
\hline & $(0.030)$ & & $(0.050)$ & $(0.071)$ \\
\hline \multirow[t]{2}{*}{ Under Six x Year2004 } & & $0.280 * * *$ & & \\
\hline & & $(0.060)$ & & \\
\hline \multirow[t]{2}{*}{ Under Six x Year2006 } & & $0.250 * * *$ & & \\
\hline & & $(0.060)$ & & \\
\hline \multirow[t]{2}{*}{ Under Six x Year2008 } & & $0.310 * * *$ & & \\
\hline & & $(0.070)$ & & \\
\hline \multirow{2}{*}{ Under Six x Year2010 } & & $0.240 * * *$ & & \\
\hline & & $(0.072)$ & & \\
\hline \multirow[t]{2}{*}{ Under Six x Year2012 } & & $0.361 * * *$ & & \\
\hline & & $(0.070)$ & & \\
\hline \multirow[t]{2}{*}{ Under Six x Year2014 } & & $0.710 * * *$ & & \\
\hline & & $(0.070)$ & & \\
\hline \multirow[t]{2}{*}{ Under Six x Year2016 } & & $0.520 * * *$ & & \\
\hline & & $(0.070)$ & & \\
\hline \multirow[t]{2}{*}{ Under Six $x$ Post $x$ Rural } & & & $-0.480 * * *$ & \\
\hline & & & $(0.050)$ & \\
\hline \multirow[t]{2}{*}{ Under Six x Post x Region 1} & & & & $0.430 * * *$ \\
\hline & & & & $(0.090)$ \\
\hline \multirow[t]{2}{*}{ Under Six x Post x Region 2} & & & & $-0.290 * * *$ \\
\hline & & & & $(0.082)$ \\
\hline \multirow[t]{2}{*}{ Under Six x Post x Region 3} & & & & -0.100 \\
\hline & & & & $(0.081)$ \\
\hline \multirow[t]{2}{*}{ Under Six x Post x Region 5} & & & & $0.572 * * *$ \\
\hline & & & & $(0.100)$ \\
\hline \multirow[t]{2}{*}{ Under Six $x$ Post $x$ Region 6} & & & & $0.370 * * *$ \\
\hline & & & & $(0.090)$ \\
\hline \multirow[t]{2}{*}{ Constant } & $1.610 * * *$ & $1.650 * * *$ & $1.500 * * *$ & $1.690 * * *$ \\
\hline & $(0.100)$ & (0.100) & $(0.101)$ & (0.101) \\
\hline Obs & 70,406 & 70,406 & 70,406 & 70,406 \\
\hline R Squared & 0.112 & 0.113 & 0.113 & 0.115 \\
\hline
\end{tabular}


Table 5. 14 Direct Health Effect - Individual Health Expenditure (Children Aged 2-10)

\begin{tabular}{|c|c|c|c|c|}
\hline & (1) & $(2)$ & (3) & (4) \\
\hline \multirow[t]{2}{*}{ Under Six } & $-0.210 * * *$ & $-0.270 * * *$ & $-0.210 * * *$ & $-0.210 * * *$ \\
\hline & $(0.040)$ & $(0.040)$ & $(0.040)$ & $(0.040)$ \\
\hline \multirow[t]{2}{*}{ Under Six x Post } & $0.390 * * *$ & & $0.770 * * *$ & $0.300 * * *$ \\
\hline & $(0.040)$ & & $(0.060)$ & (0.090) \\
\hline \multirow[t]{2}{*}{ Under Six x Year2004 } & & $0.280 * * *$ & & \\
\hline & & $(0.060)$ & & \\
\hline \multirow[t]{2}{*}{ Under Six x Year2006 } & & $0.220 * * *$ & & \\
\hline & & $(0.070)$ & & \\
\hline \multirow[t]{2}{*}{ Under Six x Year2008 } & & $0.291 * * *$ & & \\
\hline & & (0.070) & & \\
\hline \multirow[t]{2}{*}{ Under Six x Year2010 } & & $0.351 * * *$ & & \\
\hline & & $(0.070)$ & & \\
\hline \multirow[t]{2}{*}{ Under Six x Year2012 } & & $0.450 * * *$ & & \\
\hline & & $(0.080)$ & & \\
\hline \multirow[t]{2}{*}{ Under Six x Year2014 } & & $0.750 * * *$ & & \\
\hline & & $(0.080)$ & & \\
\hline \multirow[t]{2}{*}{ Under Six x Year2016 } & & $0.580 * * *$ & & \\
\hline & & $(0.080)$ & & \\
\hline \multirow{2}{*}{ Under Six x Post x Rural } & & & $-0.511 * * *$ & \\
\hline & & & $(0.061)$ & \\
\hline \multirow[t]{2}{*}{ Under Six x Post $x$ Region 1} & & & & $0.351 * * *$ \\
\hline & & & & $(0.100)$ \\
\hline \multirow[t]{2}{*}{ Under Six x Post x Region 2} & & & & $-0.360 * * *$ \\
\hline & & & & $(0.090)$ \\
\hline \multirow[t]{2}{*}{ Under Six x Post $x$ Region 3} & & & & -0.140 \\
\hline & & & & $(0.100)$ \\
\hline \multirow[t]{2}{*}{ Under Six x Post $x$ Region 5} & & & & $0.530 * * *$ \\
\hline & & & & $(0.100)$ \\
\hline \multirow[t]{2}{*}{ Under Six x Post $x$ Region 6} & & & & $0.360 * * *$ \\
\hline & & & & $(0.100)$ \\
\hline \multirow[t]{2}{*}{ Constant } & $2.650 * * *$ & $2.670 * * *$ & $2.550 * * *$ & $2.700 * * *$ \\
\hline & $(0.200)$ & $(0.200)$ & $(0.200)$ & $(0.201)$ \\
\hline Obs & 59,924 & 59,924 & 59,924 & 59,924 \\
\hline R Squared & 0.115 & 0.116 & 0.117 & 0.119 \\
\hline
\end{tabular}


Table 5. 15 Direct Health Effect - Individual Health Expenditure (Children Aged 4-7)

\begin{tabular}{|c|c|c|c|c|}
\hline & (1) & $(2)$ & (3) & (4) \\
\hline \multirow[t]{2}{*}{ Under Six } & -0.071 & $-0.120 *$ & -0.070 & -0.067 \\
\hline & $(0.070)$ & $(0.070)$ & $(0.070)$ & $(0.070)$ \\
\hline \multirow[t]{2}{*}{ Under Six x Post } & $0.170 * * *$ & & $0.380 * * *$ & 0.090 \\
\hline & $(0.050)$ & & (0.091) & $(0.102)$ \\
\hline \multirow[t]{2}{*}{ Under Six x Year2004 } & & $0.270 * * *$ & & \\
\hline & & $(0.090)$ & & \\
\hline \multirow[t]{2}{*}{ Under Six x Year2006 } & & 0.130 & & \\
\hline & & $(0.101)$ & & \\
\hline \multirow[t]{2}{*}{ Under Six x Year2008 } & & 0.068 & & \\
\hline & & $(0.101)$ & & \\
\hline \multirow[t]{2}{*}{ Under Six x Year2010 } & & $0.201^{*}$ & & \\
\hline & & $(0.100)$ & & \\
\hline \multirow{2}{*}{ Under Six x Year2012 } & & $0.280 * *$ & & \\
\hline & & $(0.100)$ & & \\
\hline \multirow[t]{2}{*}{ Under Six x Year2014 } & & $0.420 * * *$ & & \\
\hline & & $(0.100)$ & & \\
\hline \multirow[t]{2}{*}{ Under Six x Year2016 } & & $0.240 * *$ & & \\
\hline & & $(0.100)$ & & \\
\hline \multirow[t]{2}{*}{ Under Six x Post $x$ Rural } & & & $-0.270 * * *$ & \\
\hline & & & $(0.090)$ & \\
\hline \multirow[t]{2}{*}{ Under Six x Post $x$ Region 1} & & & & $0.240 *$ \\
\hline & & & & $(0.100)$ \\
\hline \multirow[t]{2}{*}{ Under Six x Post $\mathrm{x}$ Region 2} & & & & $-0.280 * *$ \\
\hline & & & & $(0.100)$ \\
\hline \multirow[t]{2}{*}{ Under Six x Post x Region 3} & & & & -0.053 \\
\hline & & & & $(0.100)$ \\
\hline \multirow[t]{2}{*}{ Under Six x Post x Region 5} & & & & $0.360 * *$ \\
\hline & & & & $(0.201)$ \\
\hline \multirow[t]{2}{*}{ Under Six x Post x Region 6} & & & & $0.381 * * *$ \\
\hline & & & & $(0.100)$ \\
\hline \multirow[t]{2}{*}{ Constant } & $1.440 * * *$ & $1.450 * * *$ & $1.380 * * *$ & $1.450 * * *$ \\
\hline & $(0.500)$ & $(0.500)$ & $(0.500)$ & $(0.500)$ \\
\hline Obs & 25,847 & 25,847 & 25,847 & 25,847 \\
\hline R Squared & 0.109 & 0.109 & 0.109 & 0.111 \\
\hline
\end{tabular}


Table 5. 16 Direct Health Effect - Outpatient Expenditure (Children Aged 0-10)

\begin{tabular}{|c|c|c|c|c|}
\hline & (1) & $(2)$ & (3) & (4) \\
\hline \multirow[t]{2}{*}{ Under Six } & $0.073 * *$ & 0.002 & $0.072 * *$ & $0.069 * *$ \\
\hline & $(0.030)$ & $(0.030)$ & $(0.030)$ & $(0.031)$ \\
\hline \multirow[t]{2}{*}{ Under Six x Post } & $0.331 * * *$ & & $0.731 * * *$ & $0.280 * * *$ \\
\hline & $(0.030)$ & & $(0.050)$ & $(0.070)$ \\
\hline \multirow[t]{2}{*}{ Under Six x Year2004 } & & $0.330 * * *$ & & \\
\hline & & $(0.050)$ & & \\
\hline \multirow[t]{2}{*}{ Under Six x Year2006 } & & $0.320 * * *$ & & \\
\hline & & $(0.060)$ & & \\
\hline \multirow[t]{2}{*}{ Under Six x Year2008 } & & $0.282 * * *$ & & \\
\hline & & $(0.060)$ & & \\
\hline \multirow[t]{2}{*}{ Under Six x Year2010 } & & $0.310 * * *$ & & \\
\hline & & $(0.060)$ & & \\
\hline \multirow[t]{2}{*}{ Under Six x Year2012 } & & $0.361 * * *$ & & \\
\hline & & $(0.060)$ & & \\
\hline \multirow[t]{2}{*}{ Under Six x Year2014 } & & $0.570 * * *$ & & \\
\hline & & $(0.060)$ & & \\
\hline \multirow[t]{2}{*}{ Under Six x Year2016 } & & $0.530 * * *$ & & \\
\hline & & (0.070) & & \\
\hline \multirow[t]{2}{*}{ Under Six $x$ Post $x$ Rural } & & & $-0.540 * * *$ & \\
\hline & & & $(0.050)$ & \\
\hline \multirow[t]{2}{*}{ Under Six x Post $x$ Region 1} & & & & $0.260 * * *$ \\
\hline & & & & $(0.080)$ \\
\hline \multirow[t]{2}{*}{ Under Six $x$ Post $x$ Region 2} & & & & $-0.460 * * *$ \\
\hline & & & & $(0.070)$ \\
\hline \multirow[t]{2}{*}{ Under Six x Post $x$ Region 3} & & & & $-0.181 * *$ \\
\hline & & & & $(0.080)$ \\
\hline \multirow[t]{2}{*}{ Under Six x Post x Region 5} & & & & $0.580 * * *$ \\
\hline & & & & (0.100) \\
\hline \multirow[t]{2}{*}{ Under Six $x$ Post $x$ Region 6} & & & & $0.400 * * *$ \\
\hline & & & & $(0.080)$ \\
\hline \multirow[t]{2}{*}{ Constant } & $1.170 * * *$ & $1.210 * * *$ & $1.050 * * *$ & $1.231 * * *$ \\
\hline & $(0.100)$ & $(0.100)$ & $(0.100)$ & (0.100) \\
\hline Obs & 70,406 & 70,406 & 70,406 & 70,406 \\
\hline R Squared & 0.128 & 0.129 & 0.131 & 0.134 \\
\hline
\end{tabular}


Table 5. 17 Direct Health Effect - Outpatient Expenditure (Children Aged 2-10)

\begin{tabular}{|c|c|c|c|c|}
\hline & (1) & $(2)$ & (3) & (4) \\
\hline Under Six & $\begin{array}{r}-0.170 * * * \\
(0.040)\end{array}$ & $\begin{array}{r}-0.240 * * * \\
(0.040)\end{array}$ & $\begin{array}{r}-0.170 * * * \\
(0.040)\end{array}$ & $\begin{array}{r}-0.180 * * * \\
(0.040)\end{array}$ \\
\hline Under Six x Post & $\begin{array}{r}0.370 * * * \\
(0.030)\end{array}$ & & $\begin{array}{r}0.801^{* * *} \\
(0.060)\end{array}$ & $\begin{array}{r}0.330 * * * \\
(0.080)\end{array}$ \\
\hline Under Six x Year2004 & & $\begin{array}{r}0.300 * * * \\
(0.060)\end{array}$ & & \\
\hline Under Six x Year2006 & & $\begin{array}{r}0.290 * * * \\
(0.063)\end{array}$ & & \\
\hline Under Six x Year2008 & & $\begin{array}{r}0.261^{* * *} \\
(0.070)\end{array}$ & & \\
\hline Under Six x Year2010 & & $\begin{array}{r}0.370 * * * \\
(0.070)\end{array}$ & & \\
\hline Under Six x Year2012 & & $\begin{array}{r}0.450 * * * \\
(0.070)\end{array}$ & & \\
\hline Under Six x Year2014 & & $\begin{array}{r}0.600 * * * \\
(0.070)\end{array}$ & & \\
\hline Under Six x Year2016 & & $\begin{array}{r}0.600 * * * \\
(0.070)\end{array}$ & & \\
\hline Under Six $x$ Post $x$ Rural & & & $\begin{array}{r}-0.570 * * * \\
(0.060)\end{array}$ & \\
\hline Under Six x Post x Region 1 & & & & $\begin{array}{r}0.250 * * * \\
(0.090)\end{array}$ \\
\hline Under Six $x$ Post $x$ Region 2 & & & & $\begin{array}{r}-0.500 * * * \\
(0.080)\end{array}$ \\
\hline Under Six x Post $x$ Region 3 & & & & $\begin{array}{c}-0.180^{*} \\
(0.090)\end{array}$ \\
\hline Under Six x Post $x$ Region 5 & & & & $\begin{array}{r}0.540 * * * \\
(0.102)\end{array}$ \\
\hline Under Six x Post $x$ Region 6 & & & & $\begin{array}{r}0.420 * * * \\
(0.090)\end{array}$ \\
\hline Constant & $\begin{array}{r}2.010 * * * \\
(0.100)\end{array}$ & $\begin{array}{r}2.030 * * * \\
(0.100)\end{array}$ & $\begin{array}{r}1.900 * * * \\
(0.103)\end{array}$ & $\begin{array}{r}2.060 * * * \\
(0.103)\end{array}$ \\
\hline Obs & 59,924 & 59,924 & 59,924 & 59,924 \\
\hline R Squared & 0.130 & 0.131 & 0.132 & 0.135 \\
\hline
\end{tabular}


Table 5. 18 Direct Health Effect - Outpatient Expenditure (Children Aged 4-7)

\begin{tabular}{|c|c|c|c|c|}
\hline & (1) & $(2)$ & (3) & (4) \\
\hline \multirow[t]{2}{*}{ Under Six } & -0.067 & $-0.120 * *$ & -0.066 & -0.063 \\
\hline & (0.060) & $(0.060)$ & $(0.060)$ & $(0.060)$ \\
\hline \multirow[t]{2}{*}{ Under Six x Post } & $0.170 * * *$ & & $0.420 * * *$ & 0.120 \\
\hline & $(0.050)$ & & $(0.080)$ & $(0.102)$ \\
\hline \multirow[t]{2}{*}{ Under Six x Year2004 } & & $0.290 * * *$ & & \\
\hline & & $(0.080)$ & & \\
\hline \multirow[t]{2}{*}{ Under Six x Year2006 } & & $0.210 * *$ & & \\
\hline & & $(0.090)$ & & \\
\hline \multirow[t]{2}{*}{ Under Six x Year2008 } & & 0.055 & & \\
\hline & & $(0.100)$ & & \\
\hline \multirow[t]{2}{*}{ Under Six x Year2010 } & & $0.210 * *$ & & \\
\hline & & $(0.104)$ & & \\
\hline \multirow{2}{*}{ Under Six x Year2012 } & & $0.280 * * *$ & & \\
\hline & & $(0.100)$ & & \\
\hline \multirow[t]{2}{*}{ Under Six x Year2014 } & & $0.290 * * *$ & & \\
\hline & & $(0.100)$ & & \\
\hline \multirow[t]{2}{*}{ Under Six x Year2016 } & & $0.270 * *$ & & \\
\hline & & $(0.105)$ & & \\
\hline \multirow[t]{2}{*}{ Under Six x Post $x$ Rural } & & & $-0.340 * * *$ & \\
\hline & & & $(0.080)$ & \\
\hline \multirow[t]{2}{*}{ Under Six x Post $x$ Region 1} & & & & 0.170 \\
\hline & & & & $(0.100)$ \\
\hline \multirow[t]{2}{*}{ Under Six x Post $\mathrm{x}$ Region 2} & & & & $-0.390 * * *$ \\
\hline & & & & $(0.100)$ \\
\hline \multirow[t]{2}{*}{ Under Six x Post x Region 3} & & & & -0.083 \\
\hline & & & & $(0.100)$ \\
\hline \multirow[t]{2}{*}{ Under Six x Post x Region 5} & & & & $0.340 * *$ \\
\hline & & & & $(0.200)$ \\
\hline \multirow[t]{2}{*}{ Under Six x Post x Region 6} & & & & $0.390 * * *$ \\
\hline & & & & $(0.1)$ \\
\hline \multirow[t]{2}{*}{ Constant } & 0.640 & 0.650 & 0.561 & 0.641 \\
\hline & $(0.500)$ & $(0.502)$ & (0.501) & $(0.501)$ \\
\hline Obs & 25,847 & 25,847 & 25,847 & 25,847 \\
\hline R Squared & 0.125 & 0.126 & 0.126 & 0.128 \\
\hline
\end{tabular}


Table 5. 19 Direct Health Effect - Inpatient Expenditure (Children Aged 0-10)

\begin{tabular}{|c|c|c|c|c|}
\hline & (1) & (2) & (3) & (4) \\
\hline \multirow[t]{2}{*}{ Under Six } & 0.015 & 0.015 & 0.015 & 0.018 \\
\hline & $(0.020)$ & $(0.020)$ & $(0.020)$ & (0.020) \\
\hline \multirow[t]{2}{*}{ Under Six x Post } & $0.061 * * *$ & & 0.039 & -0.060 \\
\hline & $(0.021)$ & & $(0.031)$ & (0.041) \\
\hline \multirow[t]{2}{*}{ Under Six x Year2004 } & & -0.001 & & \\
\hline & & (0.031) & & \\
\hline \multirow[t]{2}{*}{ Under Six x Year2006 } & & -0.032 & & \\
\hline & & $(0.040)$ & & \\
\hline \multirow[t]{2}{*}{ Under Six x Year2008 } & & $0.110 * * *$ & & \\
\hline & & $(0.040)$ & & \\
\hline \multirow[t]{2}{*}{ Under Six x Year2010 } & & -0.014 & & \\
\hline & & $(0.040)$ & & \\
\hline \multirow[t]{2}{*}{ Under Six x Year2012 } & & $0.078^{*}$ & & \\
\hline & & $(0.040)$ & & \\
\hline \multirow[t]{2}{*}{ Under Six x Year2014 } & & $0.200 * * *$ & & \\
\hline & & $(0.040)$ & & \\
\hline \multirow[t]{2}{*}{ Under Six x Year2016 } & & 0.031 & & \\
\hline & & $(0.040)$ & & \\
\hline \multirow[t]{2}{*}{ Under Six x Post $x$ Rural } & & & 0.030 & \\
\hline & & & $(0.030)$ & \\
\hline \multirow[t]{2}{*}{ Under Six x Post x Region 1} & & & & $0.260 * * *$ \\
\hline & & & & $(0.050)$ \\
\hline \multirow[t]{2}{*}{ Under Six x Post x Region 2} & & & & $0.191 * * *$ \\
\hline & & & & $(0.050)$ \\
\hline \multirow[t]{2}{*}{ Under Six x Post $x$ Region 3} & & & & $0.110 * *$ \\
\hline & & & & $(0.050)$ \\
\hline \multirow[t]{2}{*}{ Under Six x Post $x$ Region 5} & & & & 0.079 \\
\hline & & & & $(0.060)$ \\
\hline \multirow[t]{2}{*}{ Under Six x Post $x$ Region 6} & & & & 0.004 \\
\hline & & & & (0.051) \\
\hline \multirow[t]{2}{*}{ Constant } & $0.530 * * *$ & $0.530 * * *$ & $0.540 * * *$ & $0.561 * * *$ \\
\hline & $(0.070)$ & $(0.070)$ & $(0.070)$ & $(0.071)$ \\
\hline Obs & 70,406 & 70,406 & 70,406 & 70,406 \\
\hline R Squared & 0.011 & 0.012 & 0.011 & 0.012 \\
\hline
\end{tabular}


Table 5. 20 Direct Health Effect - Inpatient Expenditure (Children Aged 2-10)

\begin{tabular}{|c|c|c|c|c|}
\hline & (1) & $(2)$ & (3) & (4) \\
\hline \multirow[t]{2}{*}{ Under Six } & $-0.054 * *$ & $-0.057 * *$ & $-0.054 * *$ & $-0.052 * *$ \\
\hline & $(0.030)$ & $(0.030)$ & $(0.030)$ & $(0.030)$ \\
\hline \multirow[t]{2}{*}{ Under Six x Post } & $0.058 * *$ & & 0.027 & -0.040 \\
\hline & $(0.020)$ & & $(0.030)$ & $(0.051)$ \\
\hline \multirow[t]{2}{*}{ Under Six x Year2004 } & & 0.013 & & \\
\hline & & (0.040) & & \\
\hline \multirow[t]{2}{*}{ Under Six x Year2006 } & & -0.045 & & \\
\hline & & (0.040) & & \\
\hline \multirow[t]{2}{*}{ Under Six x Year2008 } & & $0.084 * *$ & & \\
\hline & & $(0.040)$ & & \\
\hline \multirow[t]{2}{*}{ Under Six x Year2010 } & & 0.028 & & \\
\hline & & (0.041) & & \\
\hline \multirow[t]{2}{*}{ Under Six x Year2012 } & & $0.073 *$ & & \\
\hline & & $(0.044)$ & & \\
\hline \multirow[t]{2}{*}{ Under Six x Year2014 } & & $0.200 * * *$ & & \\
\hline & & $(0.050)$ & & \\
\hline \multirow[t]{2}{*}{ Under Six x Year2016 } & & 0.015 & & \\
\hline & & $(0.050)$ & & \\
\hline \multirow[t]{2}{*}{ Under Six x Post x Rural } & & & 0.041 & \\
\hline & & & $(0.030)$ & \\
\hline \multirow[t]{2}{*}{ Under Six x Post $x$ Region 1} & & & & $0.180 * * *$ \\
\hline & & & & $(0.060)$ \\
\hline \multirow[t]{2}{*}{ Under Six x Post x Region 2} & & & & $0.180 * * *$ \\
\hline & & & & $(0.061)$ \\
\hline \multirow[t]{2}{*}{ Under Six x Post x Region 3} & & & & $0.093^{*}$ \\
\hline & & & & $(0.060)$ \\
\hline \multirow[t]{2}{*}{ Under Six x Post $x$ Region 5} & & & & 0.091 \\
\hline & & & & $(0.060)$ \\
\hline \multirow[t]{2}{*}{ Under Six x Post $x$ Region 6} & & & & -0.009 \\
\hline & & & & $(0.060)$ \\
\hline \multirow[t]{2}{*}{ Constant } & $0.820 * * *$ & $0.830 * * *$ & $0.831 * * *$ & $0.840 * * *$ \\
\hline & $(0.090)$ & $(0.090)$ & $(0.090)$ & $(0.090)$ \\
\hline Obs & 59,924 & 59,924 & 59,924 & 59,924 \\
\hline R Squared & 0.010 & 0.010 & 0.010 & 0.010 \\
\hline
\end{tabular}


Table 5. 21 Direct Health Effect - Inpatient Expenditure (Children Aged 4-7)

\begin{tabular}{|c|c|c|c|c|}
\hline & $(1)$ & $(2)$ & (3) & $(4)$ \\
\hline Under Six & $\begin{array}{l}0.0053 \\
(0.040)\end{array}$ & $\begin{array}{r}0.001 \\
(0.040)\end{array}$ & $\begin{array}{r}0.005 \\
(0.040)\end{array}$ & $\begin{array}{r}0.006 \\
(0.040)\end{array}$ \\
\hline Under Six x Post & $\begin{array}{r}0.042 \\
(0.030)\end{array}$ & & $\begin{array}{r}-0.004 \\
(0.041)\end{array}$ & $\begin{array}{c}-0.027 \\
(0.061)\end{array}$ \\
\hline Under Six x Year2004 & & $\begin{array}{r}0.017 \\
(0.050)\end{array}$ & & \\
\hline Under Six x Year2006 & & $\begin{array}{r}-0.072 \\
(0.060)\end{array}$ & & \\
\hline Under Six x Year2008 & & $\begin{array}{r}0.056 \\
(0.060)\end{array}$ & & \\
\hline Under Six x Year2010 & & $\begin{array}{r}0.053 \\
(0.060)\end{array}$ & & \\
\hline Under Six x Year2012 & & $\begin{array}{r}0.083 \\
(0.060)\end{array}$ & & \\
\hline Under Six x Year2014 & & $\begin{array}{r}0.170 * * \\
(0.070)\end{array}$ & & \\
\hline Under Six x Year2016 & & $\begin{array}{r}-0.026 \\
(0.060)\end{array}$ & & \\
\hline Under Six $x$ Post $x$ Rural & & & $\begin{array}{r}0.063 \\
(0.050)\end{array}$ & \\
\hline Under Six x Post $x$ Region 1 & & & & $\begin{array}{l}0.130 * \\
(0.080)\end{array}$ \\
\hline Under Six x Post x Region 2 & & & & $\begin{array}{r}0.100 \\
(0.070)\end{array}$ \\
\hline Under Six x Post $x$ Region 3 & & & & $\begin{array}{r}0.073 \\
(0.070)\end{array}$ \\
\hline Under Six x Post $x$ Region 5 & & & & $\begin{array}{r}0.063 \\
(0.080)\end{array}$ \\
\hline Under Six x Post $x$ Region 6 & & & & $\begin{array}{r}0.007 \\
(0.071)\end{array}$ \\
\hline Constant & $\begin{array}{r}0.841 * * * \\
(0.300)\end{array}$ & $\begin{array}{r}0.841 * * * \\
(0.301)\end{array}$ & $\begin{array}{r}0.860 * * * \\
(0.300)\end{array}$ & $\begin{array}{r}0.860 * * * \\
(0.300)\end{array}$ \\
\hline Obs & 25,847 & 25,847 & 25,847 & 25,847 \\
\hline R Squared & 0.006 & 0.007 & 0.006 & 0.006 \\
\hline
\end{tabular}


Table 5. 22 Direct Health Effect - Morbidity Last 30 days (Children Aged 0-10)

Under Six x Post x Rural

Under Six x Post x Region 1

Under Six x Post x Region 2

Under Six x Post x Region 3

Under Six x Post x Region 5

Under Six x Post x Region 6
(1)

$$
\begin{array}{r}
0.078 * * * \\
(0.020) \\
-0.028 *
\end{array}
$$$$
\text { (0.020) }
$$

Under Six x Year2006

Under Six x Year2008

(2)

(3)

Under Six$$
-0.028
$$$$
\text { (0.020) }
$$

$-0.028$

(0.020)

$-0.019$

(0.021)

$\begin{array}{rr}078 * * * & 0.078 * * * \\ (0.020) & (0.021) \\ & \\ -0.013 & -0.053 * \\ (0.020) & (0.030)\end{array}$

(0.020) (0.030)

$078 * * *$
$(0.021)$
$-0.053^{*}$
$(0.030)$
0.048

(0.041)

0.050

(0.031)

0.014

(0.031)

0.034

(0.042)

$-0.016$

(0.041)

\begin{tabular}{lrrrr} 
Constant & $0.460 * * *$ & $0.460 * * *$ & $0.450 * * *$ & $0.460 * * *$ \\
& $(0.060)$ & $(0.060)$ & $(0.060)$ & $(0.061)$ \\
& & & & \\
Obs & 15,401 & 15,401 & 15,401 & 15,401 \\
R Squared & 0.067 & 0.067 & 0.067 & 0.068 \\
\hline
\end{tabular}


Table 5. 23 Direct Health Effect - Morbidity Last 30 days (Children Aged 2-10)

Under Six

Under Six x Post

Under Six x Year2006

Under Six x Year2008

Under Six x Post x Rural

Under Six x Post x Region 1

Under Six x Post x Region 2

Under Six x Post x Region 3

Under Six x Post $x$ Region 5

Under Six x Post x Region 6
(1)

(2)

(3)

$\begin{array}{rrrr}0.025 & 0.025 & 0.025 & 0.025 \\ (0.020) & (0.020) & (0.020) & (0.020) \\ & & & \\ -0.020 & & 0.016 & -0.051 \\ (0.020) & & (0.030) & (0.041)\end{array}$

$-0.011$

(0.020)

$-0.028$

(0.020)
$-0.045 *$

(0.020) 
Table 5. 24 Direct Health Effect - Morbidity Last 30 days (Children Aged 4-7)

\begin{tabular}{|c|c|c|c|c|}
\hline & (1) & (2) & (3) & (4) \\
\hline \multirow[t]{2}{*}{ Under Six } & 0.054 & 0.054 & 0.054 & 0.054 \\
\hline & (0.031) & (0.031) & $(0.030)$ & (0.030) \\
\hline \multirow[t]{2}{*}{ Under Six x Post } & $-0.065 * *$ & & -0.052 & -0.069 \\
\hline & $(0.030)$ & & $(0.040)$ & $(0.050)$ \\
\hline \multirow[t]{2}{*}{ Under Six x Year2006 } & & $-0.059 *$ & & \\
\hline & & $(0.030)$ & & \\
\hline \multirow[t]{2}{*}{ Under Six x Year2008 } & & $-0.072 * *$ & & \\
\hline & & $(0.030)$ & & \\
\hline \multirow[t]{2}{*}{ Under Six x Post x Rural } & & & -0.017 & \\
\hline & & & $(0.041)$ & \\
\hline \multirow[t]{2}{*}{ Under Six x Post x Region 1} & & & & 0.039 \\
\hline & & & & $(0.062)$ \\
\hline \multirow[t]{2}{*}{ Under Six x Post x Region 2} & & & & 0.015 \\
\hline & & & & $(0.052)$ \\
\hline \multirow[t]{2}{*}{ Under Six x Post $x$ Region 3} & & & & -0.041 \\
\hline & & & & $(0.063)$ \\
\hline \multirow[t]{2}{*}{ Under Six x Post $x$ Region 5} & & & & 0.026 \\
\hline & & & & $(0.074)$ \\
\hline \multirow[t]{2}{*}{ Under Six x Post $x$ Region 6} & & & & -0.002 \\
\hline & & & & $(0.061)$ \\
\hline \multirow[t]{2}{*}{ Constant } & 0.210 & 0.210 & 0.200 & 0.210 \\
\hline & $(0.200)$ & $(0.200)$ & $(0.203)$ & $(0.203)$ \\
\hline Obs & 5,443 & 5,443 & 5,443 & 5,443 \\
\hline R Squared & 0.074 & 0.074 & 0.074 & 0.074 \\
\hline
\end{tabular}


Table 5. 25 Direct Health Effect - Morbidity Last 12 months (Children Aged 0-10)

\begin{tabular}{|c|c|c|c|c|}
\hline & (1) & $(2)$ & (3) & (4) \\
\hline Under Six & $\begin{array}{r}0.074^{* * *} \\
(0.020)\end{array}$ & $\begin{array}{r}0.074 * * * \\
(0.020)\end{array}$ & $\begin{array}{r}0.074 * * * \\
(0.020)\end{array}$ & $\begin{array}{r}0.074 * * * \\
(0.020)\end{array}$ \\
\hline Under Six x Post & $\begin{array}{r}-0.013 \\
(0.010)\end{array}$ & & $\begin{array}{r}-0.004 \\
(0.020)\end{array}$ & $\begin{array}{l}-0.031 \\
(0.030)\end{array}$ \\
\hline Under Six x Year2006 & & $\begin{array}{r}-0.000 \\
(0.021)\end{array}$ & & \\
\hline Under Six x Year2008 & & $\begin{array}{r}-0.026 \\
(0.020)\end{array}$ & & \\
\hline Under Six x Post x Rural & & & $\begin{array}{r}-0.011 \\
(0.020)\end{array}$ & \\
\hline Under Six x Post x Region 1 & & & & $\begin{array}{r}0.044 \\
(0.030)\end{array}$ \\
\hline Under Six x Post x Region 2 & & & & $\begin{array}{l}0.054^{*} \\
(0.030)\end{array}$ \\
\hline Under Six x Post $x$ Region 3 & & & & $\begin{array}{r}0.011 \\
(0.030)\end{array}$ \\
\hline Under Six x Post x Region 5 & & & & $\begin{array}{r}0.001 \\
(0.030)\end{array}$ \\
\hline Under Six x Post x Region 6 & & & & $\begin{array}{r}-0.023 \\
(0.030)\end{array}$ \\
\hline Constant & $\begin{array}{r}0.680 * * * \\
(0.050)\end{array}$ & $\begin{array}{r}0.680 * * * \\
(0.050)\end{array}$ & $\begin{array}{r}0.680 * * * \\
(0.060)\end{array}$ & $\begin{array}{r}0.681 * * * \\
(0.060)\end{array}$ \\
\hline Obs & 18,844 & 18,844 & 18,844 & 18,844 \\
\hline R Squared & 0.088 & 0.088 & 0.088 & 0.088 \\
\hline
\end{tabular}


Table 5. 26 Direct Health Effect - Morbidity Last 12 months (Children Aged 2-10)

\begin{tabular}{lrrrr}
\hline & $(1)$ & $(2)$ & $(3)$ & $(4)$ \\
\hline Under Six & & & & \\
& 0.026 & 0.027 & 0.027 & 0.027 \\
& $(0.020)$ & $(0.020)$ & $(0.020)$ & $(0.021)$ \\
Under Six x Post & & & & \\
& -0.011 & & 0.013 & -0.031 \\
& $(0.021)$ & & $(0.021)$ & $(0.031)$
\end{tabular}

Under Six x Year2006

0.003

(0.021)

Under Six x Year2008

$-0.026$

(0.020)

Under Six x Post x Rural

$-0.031$

(0.022)

Under Six x Post $x$ Region 1

0.055

(0.034)

Under Six $x$ Post $x$ Region 2

0.041

(0.034)

Under Six $x$ Post $x$ Region 3

0.002

(0.030)

Under Six x Post $x$ Region 5

0.005

(0.040)

Under Six $x$ Post $x$ Region 6

0.001

(0.033)

Constant

$0.861 * * * \quad 0.861 * *$

$0.854 * * *$

$0.860 * * *$

$(0.070)$

(0.070)

(0.071)

(0.071)

Obs

$15,909 \quad 15,909$

15,909

15,909

R Squared

0.093

0.093

0.093

0.093 
Table 5. 27 Direct Health Effect - Morbidity Last 12 months (Children Aged 4-7)

\begin{tabular}{|c|c|c|c|c|}
\hline & (1) & $(2)$ & (3) & (4) \\
\hline \multirow[t]{2}{*}{ Under Six } & 0.043 & 0.043 & 0.044 & 0.043 \\
\hline & $(0.031)$ & $(0.030)$ & $(0.030)$ & $(0.030)$ \\
\hline \multirow[t]{2}{*}{ Under Six x Post } & $-0.057 * *$ & & -0.052 & -0.062 \\
\hline & $(0.020)$ & & $(0.030)$ & $(0.040)$ \\
\hline \multirow[t]{2}{*}{ Under Six x Year2006 } & & -0.046 & & \\
\hline & & $(0.031)$ & & \\
\hline \multirow[t]{2}{*}{ Under Six x Year2008 } & & $-0.068 * *$ & & \\
\hline & & $(0.031)$ & & \\
\hline \multirow[t]{2}{*}{ Under Six x Post x Rural } & & & -0.006 & \\
\hline & & & $(0.031)$ & \\
\hline \multirow[t]{2}{*}{ Under Six x Post x Region 1} & & & & 0.029 \\
\hline & & & & $(0.050)$ \\
\hline \multirow[t]{2}{*}{ Under Six x Post x Region 2} & & & & 0.029 \\
\hline & & & & $(0.050)$ \\
\hline \multirow[t]{2}{*}{ Under Six x Post $x$ Region 3} & & & & -0.033 \\
\hline & & & & $(0.050)$ \\
\hline \multirow[t]{2}{*}{ Under Six x Post x Region 5} & & & & 0.019 \\
\hline & & & & $(0.061)$ \\
\hline \multirow[t]{2}{*}{ Under Six x Post $x$ Region 6} & & & & -0.009 \\
\hline & & & & $(0.052)$ \\
\hline \multirow[t]{2}{*}{ Constant } & $0.501 * *$ & $0.501 * *$ & $0.501 * *$ & $0.500 * *$ \\
\hline & $(0.200)$ & $(0.200)$ & $(0.200)$ & $(0.200)$ \\
\hline Obs & 6,553 & 6,553 & 6,553 & 6,553 \\
\hline R Squared & 0.090 & 0.090 & 0.090 & 0.091 \\
\hline
\end{tabular}


Table 5. 28 Persistent Health Effect - Health Insurance Status (Children aged 6-15)

\begin{tabular}{|c|c|c|c|}
\hline & $(1)$ & $(2)$ & (3) \\
\hline Exposure $(\mathrm{Y} / \mathrm{N})$ & $\begin{array}{r}0.009 \\
(0.007)\end{array}$ & $\begin{array}{r}-0.055^{* * *} \\
(0.010)\end{array}$ & $\begin{array}{r}-0.027 * * \\
(0.011)\end{array}$ \\
\hline Exposure Intensity (Years) & $\begin{array}{c}0.003 * \\
(0.002)\end{array}$ & $\begin{array}{l}0.003 * \\
(0.002)\end{array}$ & $\begin{array}{l}0.003 * \\
(0.002)\end{array}$ \\
\hline Exposure $x$ Rural & & $\begin{array}{r}0.082 * * * \\
(0.008)\end{array}$ & \\
\hline Exposure $\times$ Region 1 & & & $\begin{array}{r}0.056 * * * \\
(0.011)\end{array}$ \\
\hline Exposure $x$ Region 2 & & & $\begin{array}{r}0.015 \\
(0.012)\end{array}$ \\
\hline Exposure $\times$ Region 3 & & & $\begin{array}{r}0.014 \\
(0.010)\end{array}$ \\
\hline Exposure $\times$ Region 5 & & & $\begin{array}{r}0.077^{* * *} \\
(0.020)\end{array}$ \\
\hline Exposure $\times$ Region 6 & & & $\begin{array}{r}0.071 * * * \\
(0.010)\end{array}$ \\
\hline Constant & $\begin{array}{r}0.610 * * * \\
(0.030)\end{array}$ & $\begin{array}{r}0.640 * * * \\
(0.030)\end{array}$ & $\begin{array}{r}0.630 * * * \\
(0.031)\end{array}$ \\
\hline Obs & 47,678 & 47,678 & 47,678 \\
\hline R Squared & 0.104 & 0.106 & 0.105 \\
\hline
\end{tabular}


Table 5. 29 Persistent Health Effect - Health Visit (Children aged 6-15)

\begin{tabular}{|c|c|c|c|}
\hline & (1) & $(2)$ & (3) \\
\hline Exposure $(\mathrm{Y} / \mathrm{N})$ & $\begin{array}{r}0.023^{* * *} \\
(0.008)\end{array}$ & $\begin{array}{r}0.028 * * \\
(0.010)\end{array}$ & $\begin{array}{r}0.004 \\
(0.020)\end{array}$ \\
\hline Exposure Intensity (Years) & $\begin{array}{r}0.000 \\
(0.002)\end{array}$ & $\begin{array}{r}0.000 \\
(0.002)\end{array}$ & $\begin{array}{r}0.000 \\
(0.002)\end{array}$ \\
\hline Exposure $x$ Rural & & $\begin{array}{r}-0.006 \\
(0.010)\end{array}$ & \\
\hline Exposure $\times$ Region 1 & & & $\begin{array}{r}-0.005 \\
(0.021)\end{array}$ \\
\hline Exposure $x$ Region 2 & & & $\begin{array}{r}0.006 \\
(0.022)\end{array}$ \\
\hline Exposure $\times$ Region 3 & & & $\begin{array}{r}0.024 \\
(0.020)\end{array}$ \\
\hline Exposure $\times$ Region 5 & & & $\begin{array}{r}0.040 * * \\
(0.020)\end{array}$ \\
\hline Exposure $\times$ Region 6 & & & $\begin{array}{r}0.045^{* * *} \\
(0.020)\end{array}$ \\
\hline Constant & $\begin{array}{r}0.560 * * * \\
(0.030)\end{array}$ & $\begin{array}{r}0.560 * * * \\
(0.030)\end{array}$ & $\begin{array}{r}0.570 * * * \\
(0.030)\end{array}$ \\
\hline Obs & 47,678 & 47,678 & 47,678 \\
\hline R Squared & 0.046 & 0.046 & 0.047 \\
\hline
\end{tabular}


Table 5. 30 Persistent Health Effect - The number of Outpatient Visit (Children aged 6-15)

\begin{tabular}{|c|c|c|c|}
\hline & (1) & $(2)$ & (3) \\
\hline \multirow[t]{2}{*}{ Exposure $(\mathrm{Y} / \mathrm{N})$} & $0.092 * * *$ & $0.171 * * *$ & 0.012 \\
\hline & $(0.030)$ & $(0.050)$ & $(0.060)$ \\
\hline \multirow[t]{2}{*}{ Exposure Intensity (Years) } & -0.001 & -0.002 & -0.000 \\
\hline & $(0.009)$ & (0.009) & (0.009) \\
\hline \multirow[t]{2}{*}{ Exposure $\mathrm{x}$ Rural } & & $-0.094 * *$ & \\
\hline & & $(0.041)$ & \\
\hline \multirow[t]{2}{*}{ Exposure $\mathrm{x}$ Region 1} & & & -0.009 \\
\hline & & & $(0.070)$ \\
\hline \multirow[t]{2}{*}{ Exposure x Region 2} & & & 0.024 \\
\hline & & & $(0.070)$ \\
\hline \multirow[t]{2}{*}{ Exposure $\mathrm{x}$ Region 3} & & & 0.081 \\
\hline & & & $(0.060)$ \\
\hline \multirow[t]{2}{*}{ Exposure $\times$ Region 5} & & & 0.043 \\
\hline & & & $(0.080)$ \\
\hline \multirow[t]{2}{*}{ Exposure $x$ Region 6} & & & $0.270 * * *$ \\
\hline & & & $(0.070)$ \\
\hline \multirow[t]{2}{*}{ Constant } & $1.390 * * *$ & $1.360 * * *$ & $1.430 * * *$ \\
\hline & $(0.101)$ & $(0.110)$ & (0.110) \\
\hline Obs & 47,678 & 47,678 & 47,678 \\
\hline R Squared & 0.055 & 0.055 & 0.056 \\
\hline
\end{tabular}


Table 5. 31 Persistent Health Effect - The number of Inpatient Visit (Children aged 6-15)

\begin{tabular}{|c|c|c|c|}
\hline & (1) & $(2)$ & (3) \\
\hline \multirow[t]{2}{*}{ Exposure $(\mathrm{Y} / \mathrm{N})$} & 0.009 & -0.011 & 0.007 \\
\hline & $(0.007)$ & (0.009) & $(0.010)$ \\
\hline \multirow[t]{2}{*}{ Exposure Intensity (Years) } & -0.000 & -0.000 & -0.000 \\
\hline & $(0.002)$ & $(0.002)$ & $(0.002)$ \\
\hline \multirow[t]{2}{*}{ Exposure $\mathrm{x}$ Rural } & & $0.027 * * *$ & \\
\hline & & (0.008) & \\
\hline \multirow[t]{2}{*}{ Exposure $x$ Region 1} & & & -0.005 \\
\hline & & & $(0.010)$ \\
\hline \multirow[t]{2}{*}{ Exposure $x$ Region 2} & & & 0.011 \\
\hline & & & $(0.010)$ \\
\hline \multirow[t]{2}{*}{ Exposure $x$ Region 3} & & & 0.002 \\
\hline & & & $(0.010)$ \\
\hline \multirow[t]{2}{*}{ Exposure $x$ Region 5} & & & $0.028 *$ \\
\hline & & & $(0.010)$ \\
\hline \multirow[t]{2}{*}{ Exposure $x$ Region 6} & & & -0.014 \\
\hline & & & $(0.010)$ \\
\hline \multirow[t]{2}{*}{ Constant } & $0.046^{*}$ & $0.054^{* *}$ & $0.046^{*}$ \\
\hline & $(0.020)$ & $(0.020)$ & $(0.030)$ \\
\hline Obs & 47,678 & 47,678 & 47,678 \\
\hline R Squared & 0.002 & 0.003 & 0.003 \\
\hline
\end{tabular}


Table 5. 32 Persistent Health Effect - Individual Health Expenditure (Children aged 6-15)

\begin{tabular}{|c|c|c|c|}
\hline & (1) & $(2)$ & (3) \\
\hline Exposure $(\mathrm{Y} / \mathrm{N})$ & $\begin{array}{r}0.131^{* * *} \\
(0.040)\end{array}$ & $\begin{array}{r}0.231^{* * *} \\
(0.050)\end{array}$ & $\begin{array}{c}0.141^{* *} \\
(0.060)\end{array}$ \\
\hline Exposure Intensity (Years) & $\begin{array}{r}0.036 * * * \\
(0.009)\end{array}$ & $\begin{array}{r}0.035^{* * *} \\
(0.009)\end{array}$ & $\begin{array}{r}0.037^{* * *} \\
(0.009)\end{array}$ \\
\hline Exposure $x$ Rural & & $\begin{array}{r}-0.120 * * * \\
(0.040)\end{array}$ & \\
\hline Exposure $x$ Region 1 & & & $\begin{array}{l}-0.017 \\
(0.060)\end{array}$ \\
\hline Exposure $x$ Region 2 & & & $\begin{array}{r}-0.370 * * * \\
(0.060)\end{array}$ \\
\hline Exposure $x$ Region 3 & & & $\begin{array}{r}-0.131^{* *} \\
(0.062)\end{array}$ \\
\hline Exposure $x$ Region 5 & & & $\begin{array}{r}0.310 * * * \\
(0.070)\end{array}$ \\
\hline Exposure $x$ Region 6 & & & $\begin{array}{r}0.340 * * * \\
(0.064)\end{array}$ \\
\hline Constant & $\begin{array}{r}1.290 * * * \\
(0.100)\end{array}$ & $\begin{array}{r}1.270 * * * \\
(0.100)\end{array}$ & $\begin{array}{r}1.310^{* * * *} \\
(0.101)\end{array}$ \\
\hline $\begin{array}{l}\text { Obs } \\
\text { R Squared }\end{array}$ & 79,536 & 79,536 & 79,536 \\
\hline R Squared & 0.063 & 0.063 & 0.066 \\
\hline
\end{tabular}


Table 5. 33 Persistent Health Effect - Outpatient Expenditure (Children aged 6-15)

\begin{tabular}{|c|c|c|c|}
\hline & $(1)$ & $(2)$ & (3) \\
\hline Exposure $(\mathrm{Y} / \mathrm{N})$ & $\begin{array}{r}0.110^{* * *} \\
(0.030)\end{array}$ & $\begin{array}{r}0.270 * * * \\
(0.040)\end{array}$ & $\begin{array}{r}0.088 \\
(0.050)\end{array}$ \\
\hline Exposure Intensity (Years) & $\begin{array}{r}0.037^{* * *} \\
(0.008)\end{array}$ & $\begin{array}{r}0.036 * * * \\
(0.008)\end{array}$ & $\begin{array}{r}0.038 * * * \\
(0.008)\end{array}$ \\
\hline Exposure $x$ Rural & & $\begin{array}{r}-0.210 * * * \\
(0.031)\end{array}$ & \\
\hline Exposure $\times$ Region 1 & & & $\begin{array}{r}0.014 \\
(0.061)\end{array}$ \\
\hline Exposure $\times$ Region 2 & & & $\begin{array}{r}-0.381 * * * \\
(0.061)\end{array}$ \\
\hline Exposure $\times$ Region 3 & & & $\begin{array}{c}-0.101^{*} \\
(0.050)\end{array}$ \\
\hline Exposure $\times$ Region 5 & & & $\begin{array}{r}0.370 * * * \\
(0.060)\end{array}$ \\
\hline Exposure $x$ Region 6 & & & $\begin{array}{r}0.400 * * * \\
(0.061)\end{array}$ \\
\hline Constant & $\begin{array}{r}1.010^{* * *} \\
(0.091)\end{array}$ & $\begin{array}{r}0.970 * * * \\
(0.091)\end{array}$ & $\begin{array}{r}1.040 * * * \\
(0.090)\end{array}$ \\
\hline Obs & 79,536 & 79,536 & 79,536 \\
\hline R Squared & 0.076 & 0.077 & 0.081 \\
\hline
\end{tabular}


Table 5. 34 Persistent Health Effect - Inpatient Expenditure (Children aged 6-15)

\begin{tabular}{|c|c|c|c|}
\hline & (1) & $(2)$ & (3) \\
\hline \multirow[t]{2}{*}{ Exposure $(\mathrm{Y} / \mathrm{N})$} & 0.030 & -0.041 & $0.058 *$ \\
\hline & (0.020) & $(0.030)$ & $(0.030)$ \\
\hline \multirow[t]{2}{*}{ Exposure Intensity (Years) } & -0.001 & -0.001 & -0.001 \\
\hline & (0.005) & $(0.005)$ & $(0.005)$ \\
\hline \multirow[t]{2}{*}{ Exposure $x$ Rural } & & $0.091 * * *$ & \\
\hline & & $(0.020)$ & \\
\hline \multirow[t]{2}{*}{ Exposure $x$ Region 1} & & & -0.027 \\
\hline & & & $(0.040)$ \\
\hline \multirow[t]{2}{*}{ Exposure $x$ Region 2} & & & 0.008 \\
\hline & & & $(0.041)$ \\
\hline \multirow[t]{2}{*}{ Exposure $x$ Region 3} & & & -0.030 \\
\hline & & & $(0.041)$ \\
\hline \multirow[t]{2}{*}{ Exposure $x$ Region 5} & & & -0.057 \\
\hline & & & $(0.040)$ \\
\hline \multirow[t]{2}{*}{ Exposure $x$ Region 6} & & & $-0.062^{*}$ \\
\hline & & & $(0.040)$ \\
\hline \multirow[t]{2}{*}{ Constant } & $0.330 * * *$ & $0.340 * * *$ & $0.310 * * *$ \\
\hline & $(0.060)$ & $(0.060)$ & $(0.060)$ \\
\hline Obs & 79,536 & 79,536 & 79,536 \\
\hline R Squared & 0.003 & 0.003 & 0.003 \\
\hline
\end{tabular}


Table 5. 35 Persistent Health Effect - Morbidity Status last 30 days (Children aged 6-15)

\begin{tabular}{|c|c|c|c|}
\hline & $(1)$ & $(2)$ & (3) \\
\hline \multirow[t]{2}{*}{ Exposure $(\mathrm{Y} / \mathrm{N})$} & 0.044 & $0.070 *$ & 0.060 \\
\hline & $(0.030)$ & $(0.040)$ & $(0.040)$ \\
\hline \multirow[t]{2}{*}{ Exposure Intensity (Years) } & -0.002 & -0.002 & -0.002 \\
\hline & (0.010) & $(0.010)$ & $(0.010)$ \\
\hline \multirow[t]{2}{*}{ Exposure $\mathrm{x}$ Rural } & & -0.033 & \\
\hline & & $(0.030)$ & \\
\hline \multirow[t]{2}{*}{ Exposure $x$ Region 1} & & & -0.024 \\
\hline & & & $(0.040)$ \\
\hline \multirow[t]{2}{*}{ Exposure $x$ Region 2} & & & -0.026 \\
\hline & & & $(0.040)$ \\
\hline \multirow[t]{2}{*}{ Exposure $x$ Region 3} & & & -0.027 \\
\hline & & & $(0.042)$ \\
\hline \multirow[t]{2}{*}{ Exposure $x$ Region 5} & & & 0.037 \\
\hline & & & $(0.053)$ \\
\hline \multirow[t]{2}{*}{ Exposure $x$ Region 6} & & & -0.024 \\
\hline & & & $(0.050)$ \\
\hline \multirow[t]{2}{*}{ Constant } & $0.580 * * *$ & $0.580 * * *$ & $0.580 * * *$ \\
\hline & $(0.050)$ & $(0.050)$ & $(0.050)$ \\
\hline Obs & 21,077 & 21,077 & 21,077 \\
\hline R Squared & 0.063 & 0.063 & 0.063 \\
\hline
\end{tabular}


Table 5. 36 Persistent Health Effect - Morbidity Status last 12 months (Children aged 6-15)

\begin{tabular}{|c|c|c|c|}
\hline & (1) & (2) & (3) \\
\hline \multirow[t]{2}{*}{ Exposure $(\mathrm{Y} / \mathrm{N})$} & 0.032 & $0.057^{*}$ & 0.041 \\
\hline & $(0.030)$ & $(0.030)$ & $(0.040)$ \\
\hline \multirow[t]{2}{*}{ Exposure Intensity (Years) } & 0.002 & 0.002 & 0.002 \\
\hline & $(0.010)$ & (0.010) & $(0.010)$ \\
\hline \multirow[t]{2}{*}{ Exposure $x$ Rural } & & -0.032 & \\
\hline & & $(0.030)$ & \\
\hline \multirow[t]{2}{*}{ Exposure $\times$ Region 1} & & & -0.031 \\
\hline & & & $(0.040)$ \\
\hline \multirow[t]{2}{*}{ Exposure $x$ Region 2} & & & -0.025 \\
\hline & & & $(0.040)$ \\
\hline \multirow[t]{2}{*}{ Exposure $x$ Region 3} & & & -0.007 \\
\hline & & & $(0.041)$ \\
\hline \multirow[t]{2}{*}{ Exposure $\times$ Region 5} & & & 0.004 \\
\hline & & & $(0.050)$ \\
\hline \multirow[t]{2}{*}{ Exposure $x$ Region 6} & & & 0.008 \\
\hline & & & $(0.040)$ \\
\hline \multirow[t]{2}{*}{ Constant } & $0.730 * * *$ & $0.730 * * *$ & $0.731 * * *$ \\
\hline & $(0.050)$ & $(0.050)$ & $(0.050)$ \\
\hline Obs & 23,745 & 23,745 & 23,745 \\
\hline R Squared & 0.074 & 0.074 & 0.074 \\
\hline
\end{tabular}


Table 5. 37 Spill Over Health Effect - Health Insurance Status (Children Aged 6-15)

\begin{tabular}{|c|c|c|c|c|}
\hline & (1) & $(2)$ & (3) & (4) \\
\hline Under Six & $\begin{array}{r}-0.064^{* * *} \\
(0.010)\end{array}$ & $\begin{array}{r}-0.064 * * * \\
(0.010)\end{array}$ & $\begin{array}{r}-0.061 * * * \\
(0.010)\end{array}$ & $\begin{array}{r}-0.058 * * * \\
(0.010)\end{array}$ \\
\hline Under Six x Post & $\begin{array}{r}0.039 * * * \\
(0.010)\end{array}$ & & $\begin{array}{r}-0.001 \\
(0.010)\end{array}$ & $\begin{array}{r}0.073^{* * *} \\
(0.020)\end{array}$ \\
\hline Under Six x Year2006 & & $\begin{array}{r}0.045^{* * *} \\
(0.020)\end{array}$ & & \\
\hline Under Six x Year2008 & & $\begin{array}{r}0.044^{* * *} \\
(0.020)\end{array}$ & & \\
\hline Under Six x Year2010 & & $\begin{array}{r}0.050 * * * \\
(0.021)\end{array}$ & & \\
\hline Under Six x Year2012 & & $\begin{array}{r}0.042 * * * \\
(0.011)\end{array}$ & & \\
\hline Under Six x Year2014 & & $\begin{array}{r}0.034^{* *} \\
(0.012)\end{array}$ & & \\
\hline Under Six x Year2016 & & $\begin{array}{r}0.018 \\
(0.013)\end{array}$ & & \\
\hline Under Six x Post x Rural & & & $\begin{array}{r}0.051^{* * *} \\
(0.008)\end{array}$ & \\
\hline Under Six x Post $x$ Region 1 & & & & $\begin{array}{r}-0.055^{* * *} \\
(0.010)\end{array}$ \\
\hline Under Six x Post x Region 2 & & & & $\begin{array}{r}-0.004 \\
(0.010)\end{array}$ \\
\hline Under Six x Post x Region 3 & & & & $\begin{array}{r}-0.044^{* * *} \\
(0.010)\end{array}$ \\
\hline Under Six x Post x Region 5 & & & & $\begin{array}{r}-0.058^{* * *} \\
(0.020)\end{array}$ \\
\hline Under Six x Post x Region 6 & & & & $\begin{array}{r}-0.051 * * * \\
(0.020)\end{array}$ \\
\hline Constant & $\begin{array}{r}0.410 * * * \\
(0.040)\end{array}$ & $\begin{array}{r}0.410 * * * \\
(0.040)\end{array}$ & $\begin{array}{r}0.420 * * * \\
(0.040)\end{array}$ & $\begin{array}{r}0.394 * * * \\
(0.040)\end{array}$ \\
\hline Obs & 47,678 & 47,678 & 47,678 & 47,678 \\
\hline R Squared & 0.104 & 0.104 & 0.105 & 0.105 \\
\hline
\end{tabular}


Table 5. 38 Spill Over Health Effect - Health Visit (Children Aged 6-15)

\begin{tabular}{|c|c|c|c|c|}
\hline & (1) & $(2)$ & (3) & (4) \\
\hline \multirow[t]{2}{*}{ Under Six } & -0.016 & -0.015 & -0.016 & -0.018 \\
\hline & (0.010) & (0.010) & (0.010) & (0.010) \\
\hline \multirow[t]{2}{*}{ Under Six x Post } & 0.011 & & 0.019 & -0.006 \\
\hline & (0.010) & & (0.010) & $(0.022)$ \\
\hline \multirow[t]{2}{*}{ Under Six x Year2006 } & & 0.015 & & \\
\hline & & $(0.020)$ & & \\
\hline \multirow[t]{2}{*}{ Under Six x Year2008 } & & 0.008 & & \\
\hline & & $(0.020)$ & & \\
\hline \multirow[t]{2}{*}{ Under Six x Year2010 } & & 0.011 & & \\
\hline & & $(0.020)$ & & \\
\hline \multirow[t]{2}{*}{ Under Six x Year2012 } & & 0.000 & & \\
\hline & & $(0.020)$ & & \\
\hline \multirow[t]{2}{*}{ Under Six x Year2014 } & & 0.004 & & \\
\hline & & $(0.021)$ & & \\
\hline \multirow[t]{2}{*}{ Under Six x Year2016 } & & 0.025 & & \\
\hline & & $(0.021)$ & & \\
\hline \multirow[t]{2}{*}{ Under Six x Post $x$ Rural } & & & -0.009 & \\
\hline & & & (0.010) & \\
\hline \multirow[t]{2}{*}{ Under Six $x$ Post $x$ Region 1} & & & & 0.011 \\
\hline & & & & $(0.020)$ \\
\hline \multirow[t]{2}{*}{ Under Six x Post x Region 2} & & & & 0.016 \\
\hline & & & & $(0.020)$ \\
\hline \multirow[t]{2}{*}{ Under Six $x$ Post $x$ Region 3} & & & & 0.006 \\
\hline & & & & $(0.021)$ \\
\hline \multirow[t]{2}{*}{ Under Six x Post $x$ Region 5} & & & & $0.063 * * *$ \\
\hline & & & & $(0.020)$ \\
\hline \multirow[t]{2}{*}{ Under Six x Post x Region 6} & & & & 0.028 \\
\hline & & & & $(0.020)$ \\
\hline \multirow[t]{2}{*}{ Constant } & $0.750 * * *$ & $0.750 * * *$ & $0.750 * * *$ & $0.760 * * *$ \\
\hline & $(0.040)$ & $(0.040)$ & $(0.041)$ & $(0.041)$ \\
\hline Obs & 47,678 & 47,678 & 47,678 & 47,678 \\
\hline R Squared & 0.046 & 0.046 & 0.046 & 0.046 \\
\hline
\end{tabular}


Table 5. 39 Spill Over Health Effect - The number of Outpatient Visit (Children Aged 6-15)

\begin{tabular}{|c|c|c|c|c|}
\hline & (1) & $(2)$ & (3) & (4) \\
\hline Under Six & $\begin{array}{r}-0.002 \\
(0.060)\end{array}$ & $\begin{array}{r}-0.002 \\
(0.060)\end{array}$ & $\begin{array}{r}-0.005 \\
(0.060)\end{array}$ & $\begin{array}{r}-0.011 \\
(0.060)\end{array}$ \\
\hline Under Six x Post & $\begin{array}{r}-0.012 \\
(0.050)\end{array}$ & & $\begin{array}{r}0.032 \\
(0.060)\end{array}$ & $\begin{array}{l}-0.100 \\
(0.060)\end{array}$ \\
\hline Under Six x Year2006 & & $\begin{array}{r}-0.027 \\
(0.061)\end{array}$ & & \\
\hline Under Six x Year2008 & & $\begin{array}{r}-0.038 \\
(0.060)\end{array}$ & & \\
\hline Under Six x Year2010 & & $\begin{array}{r}0.046 \\
(0.070)\end{array}$ & & \\
\hline Under Six x Year2012 & & $\begin{array}{r}-0.045 \\
(0.060)\end{array}$ & & \\
\hline Under Six x Year2014 & & $\begin{array}{r}-0.021 \\
(0.071)\end{array}$ & & \\
\hline Under Six x Year2016 & & $\begin{array}{r}0.017 \\
(0.062)\end{array}$ & & \\
\hline Under Six x Post x Rural & & & $\begin{array}{r}-0.055 \\
(0.052)\end{array}$ & \\
\hline Under Six x Post $x$ Region 1 & & & & $\begin{array}{l}0.100 * \\
(0.060)\end{array}$ \\
\hline Under Six x Post $x$ Region 2 & & & & $\begin{array}{r}0.120 * * \\
(0.060)\end{array}$ \\
\hline Under Six $x$ Post $x$ Region 3 & & & & $\begin{array}{r}0.030 \\
(0.060)\end{array}$ \\
\hline Under Six $x$ Post $x$ Region 5 & & & & $\begin{array}{l}0.160 * \\
(0.091)\end{array}$ \\
\hline Under Six $x$ Post $x$ Region 6 & & & & $\begin{array}{r}0.150 \\
(0.090)\end{array}$ \\
\hline Constant & $\begin{array}{r}2.090 * * * \\
(0.200)\end{array}$ & $\begin{array}{r}2.090 * * * \\
(0.200)\end{array}$ & $\begin{array}{r}2.084 * * * \\
(0.200)\end{array}$ & $\begin{array}{r}2.120 * * * \\
(0.200)\end{array}$ \\
\hline Obs & 47,678 & 47,678 & 47,678 & 47,678 \\
\hline R Squared & 0.055 & 0.055 & 0.055 & 0.055 \\
\hline
\end{tabular}


Table 5. 40 Spill Over Health Effect - The number of Inpatient Visit (Children Aged 6-15)

\begin{tabular}{|c|c|c|c|c|}
\hline & (1) & $(2)$ & (3) & (4) \\
\hline Under Six & $\begin{array}{r}-0.001 \\
(0.02)\end{array}$ & $\begin{array}{r}-0.001 \\
(0.02)\end{array}$ & $\begin{array}{r}-0.001 \\
(0.02)\end{array}$ & $\begin{array}{r}-0.001 \\
(0.02)\end{array}$ \\
\hline Under Six x Post & $\begin{array}{r}0.000 \\
(0.009)\end{array}$ & & $\begin{array}{r}-0.004 \\
(0.010)\end{array}$ & $\begin{array}{l}-0.000 \\
(0.010)\end{array}$ \\
\hline Under Six x Year2006 & & $\begin{array}{r}-0.005 \\
(0.0)\end{array}$ & & \\
\hline Under Six x Year2008 & & $\begin{array}{r}-0.005 \\
(0.010)\end{array}$ & & \\
\hline Under Six x Year2010 & & $\begin{array}{r}0.001 \\
(0.012)\end{array}$ & & \\
\hline Under Six x Year2012 & & $\begin{array}{r}-0.009 \\
(0.010)\end{array}$ & & \\
\hline Under Six x Year2014 & & $\begin{array}{r}0.026 \\
(0.020)\end{array}$ & & \\
\hline Under Six x Year2016 & & $\begin{array}{r}-0.000 \\
(0.010)\end{array}$ & & \\
\hline Under Six x Post x Rural & & & $\begin{array}{r}0.006 \\
(0.009)\end{array}$ & \\
\hline Under Six x Post x Region 1 & & & & $\begin{array}{l}-0.004 \\
(0.010)\end{array}$ \\
\hline Under Six x Post x Region 2 & & & & $\begin{array}{r}-0.001 \\
(0.010)\end{array}$ \\
\hline Under Six x Post x Region 3 & & & & $\begin{array}{l}-0.000 \\
(0.010)\end{array}$ \\
\hline Under Six x Post x Region 5 & & & & $\begin{array}{r}0.057 \\
(0.040)\end{array}$ \\
\hline Under Six x Post x Region 6 & & & & $\begin{array}{l}-0.019 \\
(0.010)\end{array}$ \\
\hline Constant & $\begin{array}{r}0.044 \\
(0.030)\end{array}$ & $\begin{array}{r}0.044 \\
(0.030)\end{array}$ & $\begin{array}{r}0.045 \\
(0.030)\end{array}$ & $\begin{array}{r}0.043 \\
(0.030)\end{array}$ \\
\hline Obs & 47,678 & 47,678 & 47,678 & 47,678 \\
\hline R Squared & 0.002 & 0.002 & 0.002 & 0.003 \\
\hline
\end{tabular}


Table 5. 41 Spill Over Health Effect - Individual Health Expenditure (Children Aged 6-15)

\begin{tabular}{|c|c|c|c|c|}
\hline & (1) & (2) & (3) & $(4)$ \\
\hline Under Six & $\begin{array}{r}0.025 \\
(0.030)\end{array}$ & $\begin{array}{r}0.034 \\
(0.040)\end{array}$ & $\begin{array}{r}0.022 \\
(0.030)\end{array}$ & $\begin{array}{r}0.009 \\
(0.030)\end{array}$ \\
\hline Under Six x Post & $\begin{array}{r}-0.010 \\
(0.031)\end{array}$ & & $\begin{array}{r}0.056 \\
(0.060)\end{array}$ & $\begin{array}{c}-0.012 \\
(0.070)\end{array}$ \\
\hline Under Six x Year2004 & & $\begin{array}{r}-0.040 \\
(0.052)\end{array}$ & & \\
\hline Under Six x Year2006 & & $\begin{array}{r}-0.064 \\
(0.050)\end{array}$ & & \\
\hline Under Six x Year2008 & & $\begin{array}{r}0.016 \\
(0.060)\end{array}$ & & \\
\hline Under Six x Year2010 & & $\begin{array}{r}-0.037 \\
(0.070)\end{array}$ & & \\
\hline Under Six x Year2012 & & $\begin{array}{r}-0.026 \\
(0.070)\end{array}$ & & \\
\hline Under Six x Year2014 & & $\begin{array}{r}-0.035 \\
(0.070)\end{array}$ & & \\
\hline Under Six x Year2016 & & $\begin{array}{r}0.039 \\
(0.070)\end{array}$ & & \\
\hline Under Six x Post x Rural & & & $\begin{array}{r}-0.084 \\
(0.060)\end{array}$ & \\
\hline Under Six x Post x Region 1 & & & & $\begin{array}{r}0.010 \\
(0.090)\end{array}$ \\
\hline Under Six x Post x Region 2 & & & & $\begin{array}{r}-0.190 * * \\
(0.080)\end{array}$ \\
\hline Under Six x Post $x$ Region 3 & & & & $\begin{array}{c}-0.097 \\
(0.081)\end{array}$ \\
\hline Under Six x Post x Region 5 & & & & $\begin{array}{r}0.330 * * * \\
(0.100)\end{array}$ \\
\hline Under Six x Post x Region 6 & & & & $\begin{array}{r}0.200 * * \\
(0.091)\end{array}$ \\
\hline Constant & $\begin{array}{r}1.901^{* * *} \\
(0.100)\end{array}$ & $\begin{array}{r}1.901^{* * *} \\
(0.100)\end{array}$ & $\begin{array}{r}1.890 * * * \\
(0.100)\end{array}$ & $\begin{array}{r}1.910 * * * \\
(0.100)\end{array}$ \\
\hline Obs & 79,536 & 79,536 & 79,536 & 79,536 \\
\hline R Squared & 0.062 & 0.062 & 0.062 & 0.063 \\
\hline
\end{tabular}


Table 5. 42 Spill Over Health Effect - Outpatient Expenditure (Children Aged 6-15)

\begin{tabular}{|c|c|c|c|c|}
\hline & (1) & (2) & (3) & (4) \\
\hline Under Six & $\begin{array}{r}0.011 \\
(0.030)\end{array}$ & $\begin{array}{r}0.022 \\
(0.030)\end{array}$ & $\begin{array}{r}0.007 \\
(0.030)\end{array}$ & $\begin{array}{l}-0.006 \\
(0.030)\end{array}$ \\
\hline Under Six x Post & $\begin{array}{r}-0.017 \\
(0.030)\end{array}$ & & $\begin{array}{r}0.060 \\
(0.050)\end{array}$ & $\begin{array}{c}-0.048 \\
(0.060)\end{array}$ \\
\hline Under Six x Year2004 & & $\begin{array}{r}-0.046 \\
(0.040)\end{array}$ & & \\
\hline Under Six x Year2006 & & $\begin{array}{r}-0.033 \\
(0.050)\end{array}$ & & \\
\hline Under Six x Year2008 & & $\begin{array}{r}0.002 \\
(0.050)\end{array}$ & & \\
\hline Under Six x Year2010 & & $\begin{array}{r}-0.036 \\
(0.061)\end{array}$ & & \\
\hline Under Six x Year2012 & & $\begin{array}{r}-0.036 \\
(0.060)\end{array}$ & & \\
\hline Under Six x Year2014 & & $\begin{array}{r}-0.047 \\
(0.060)\end{array}$ & & \\
\hline Under Six x Year2016 & & $\begin{array}{r}-0.013 \\
(0.070)\end{array}$ & & \\
\hline Under Six x Post x Rural & & & $\begin{array}{c}-0.097^{*} \\
(0.050)\end{array}$ & \\
\hline Under Six x Post x Region 1 & & & & $\begin{array}{r}0.040 \\
(0.080)\end{array}$ \\
\hline Under Six x Post $x$ Region 2 & & & & $\begin{array}{r}-0.160 * * \\
(0.070)\end{array}$ \\
\hline Under Six x Post $x$ Region 3 & & & & $\begin{array}{l}-0.082 \\
(0.070)\end{array}$ \\
\hline Under Six x Post $x$ Region 5 & & & & $\begin{array}{r}0.370 * * * \\
(0.100)\end{array}$ \\
\hline Under Six $x$ Post $x$ Region 6 & & & & $\begin{array}{r}0.260 * * * \\
(0.081)\end{array}$ \\
\hline Constant & $\begin{array}{r}1.621^{* * *} \\
(0.100)\end{array}$ & $\begin{array}{r}1.621^{* * *} \\
(0.100)\end{array}$ & $\begin{array}{r}1.614^{* * *} \\
(0.100)\end{array}$ & $\begin{array}{r}1.630 * * * \\
(0.100)\end{array}$ \\
\hline Obs & 79,536 & 79,536 & 79,536 & 79,536 \\
\hline R Squared & 0.075 & 0.075 & 0.075 & 0.077 \\
\hline
\end{tabular}


Table 5. 43 Spill Over Health Effect - Inpatient Expenditure (Children Aged 6-15)

\begin{tabular}{|c|c|c|c|c|}
\hline & (1) & $(2)$ & (3) & (4) \\
\hline \multirow[t]{2}{*}{ Under Six } & 0.026 & 0.021 & 0.026 & 0.028 \\
\hline & $(0.020)$ & $(0.020)$ & $(0.020)$ & $(0.020)$ \\
\hline \multirow[t]{2}{*}{ Under Six x Post } & 0.004 & & 0.0068 & 0.029 \\
\hline & $(0.020)$ & & $(0.030)$ & $(0.040)$ \\
\hline \multirow[t]{2}{*}{ Under Six x Year2004 } & & 0.025 & & \\
\hline & & $(0.032)$ & & \\
\hline \multirow[t]{2}{*}{ Under Six x Year2006 } & & -0.026 & & \\
\hline & & $(0.030)$ & & \\
\hline \multirow[t]{2}{*}{ Under Six x Year2008 } & & 0.025 & & \\
\hline & & $(0.032)$ & & \\
\hline \multirow[t]{2}{*}{ Under Six x Year2010 } & & 0.005 & & \\
\hline & & $(0.043)$ & & \\
\hline \multirow[t]{2}{*}{ Under Six x Year2012 } & & 0.002 & & \\
\hline & & $(0.032)$ & & \\
\hline \multirow[t]{2}{*}{ Under Six x Year2014 } & & 0.003 & & \\
\hline & & $(0.041)$ & & \\
\hline \multirow[t]{2}{*}{ Under Six x Year2016 } & & 0.053 & & \\
\hline & & $(0.040)$ & & \\
\hline \multirow[t]{2}{*}{ Under Six $x$ Post $x$ Rural } & & & -0.003 & \\
\hline & & & $(0.030)$ & \\
\hline \multirow[t]{2}{*}{ Under Six x Post x Region 1} & & & & -0.019 \\
\hline & & & & $(0.050)$ \\
\hline \multirow[t]{2}{*}{ Under Six x Post x Region 2} & & & & -0.014 \\
\hline & & & & $(0.050)$ \\
\hline \multirow[t]{2}{*}{ Under Six x Post x Region 3} & & & & -0.017 \\
\hline & & & & $(0.050)$ \\
\hline \multirow[t]{2}{*}{ Under Six x Post x Region 5} & & & & -0.052 \\
\hline & & & & $(0.050)$ \\
\hline \multirow[t]{2}{*}{ Under Six x Post x Region 6} & & & & -0.061 \\
\hline & & & & $(0.050)$ \\
\hline \multirow[t]{2}{*}{ Constant } & $0.300 * * *$ & $0.310 * * *$ & $0.300 * * *$ & $0.301 * * *$ \\
\hline & $(0.080)$ & $(0.080)$ & $(0.080)$ & $(0.080)$ \\
\hline Obs & 79,536 & 79,536 & 79,536 & 79,536 \\
\hline R Squared & 0.003 & 0.003 & 0.003 & 0.003 \\
\hline
\end{tabular}


Table 5. 44 Spill Over Health Effect - Morbidity Status last 30 days (Children Aged 6-15)

(1)

Under Six

Under Six x Post

Under Six x Year2006

Under Six x Year2008

Under Six x Post x Rural

Under Six x Post x Region 1

Under Six x Post x Region 2

Under Six x Post x Region 3

Under Six x Post x Region 5

Under Six x Post x Region 6

(2)

(3)

(4)

$\begin{array}{rrrr}-0.044^{* *} & -0.044^{* *} & -0.045^{* *} & -0.051^{* * *} \\ (0.021) & (0.021) & (0.022) & (0.022) \\ & & & \\ -0.023 & & 0.014 & -0.071^{* *} \\ (0.010) & & (0.020) & (0.030)\end{array}$

$-0.0062$

(0.02)

$-0.041^{* *}$

(0.020)

$-0.044 *$

(0.020)

$0.088^{* * *}$

(0.030)

0.019

(0.030)

0.038

(0.030)

$0.140 * * *$

(0.041)

0.048

(0.040)
$0.681 * * *$

(0.070)
$0.681 * *$

(0.070)

$0.680 * * *$

(0.070)

$0.700 * * *$

(0.070)

Obs

21,077

21,077

21,077

0.063

21,077

R Squared 
Table 5. 45 Spill Over Health Effect - Morbidity Status last 12 months (Children Aged 6-15)

\begin{tabular}{|c|c|c|c|c|}
\hline & (1) & $(2)$ & (3) & (4) \\
\hline \multirow[t]{2}{*}{ Under Six } & -0.028 & -0.029 & -0.030 & $-0.036^{*}$ \\
\hline & $(0.021)$ & $(0.021)$ & $(0.020)$ & $(0.020)$ \\
\hline \multirow[t]{2}{*}{ Under Six x Post } & -0.022 & & 0.024 & $-0.085 * * *$ \\
\hline & $(0.010)$ & & $(0.020)$ & $(0.030)$ \\
\hline \multirow[t]{2}{*}{ Under Six x Year2006 } & & -0.006 & & \\
\hline & & $(0.020)$ & & \\
\hline \multirow[t]{2}{*}{ Under Six x Year2008 } & & $-0.040 * *$ & & \\
\hline & & $(0.020)$ & & \\
\hline \multirow[t]{2}{*}{ Under Six x Post $x$ Rural } & & & $-0.056 * *$ & \\
\hline & & & $(0.020)$ & \\
\hline \multirow[t]{2}{*}{ Under Six x Post $x$ Region 1} & & & & $0.090 * * *$ \\
\hline & & & & $(0.030)$ \\
\hline \multirow[t]{2}{*}{ Under Six x Post x Region 2} & & & & 0.046 \\
\hline & & & & (0.031) \\
\hline \multirow[t]{2}{*}{ Under Six x Post $x$ Region 3} & & & & $0.057^{*}$ \\
\hline & & & & $(0.032)$ \\
\hline \multirow[t]{2}{*}{ Under Six $x$ Post $x$ Region 5} & & & & $0.140 * * *$ \\
\hline & & & & $(0.040)$ \\
\hline \multirow[t]{2}{*}{ Under Six x Post $\times$ Region 6} & & & & $0.076 * *$ \\
\hline & & & & $(0.030)$ \\
\hline \multirow[t]{2}{*}{ Constant } & $0.850 * * *$ & $0.850 * * *$ & $0.840 * * *$ & $0.860 * * *$ \\
\hline & $(0.070)$ & $(0.070)$ & $(0.071)$ & $(0.071)$ \\
\hline Obs & 23,745 & 23,745 & 23,745 & 23,745 \\
\hline R Squared & 0.074 & 0.074 & 0.074 & 0.074 \\
\hline
\end{tabular}


Table 5. 46 Persistent Effect on Individual Education Expenditure (Children aged 6-15 with previously exposing to $\mathrm{FHI}$ )

\begin{tabular}{|c|c|c|c|}
\hline & (1) & $(2)$ & (3) \\
\hline Exposure $(\mathrm{Y} / \mathrm{N})$ & $\begin{array}{r}0.101 * * * \\
(0.030)\end{array}$ & $\begin{array}{c}-0.022 \\
(0.040)\end{array}$ & $\begin{array}{r}-0.172 * * * \\
(0.051)\end{array}$ \\
\hline Exposure Intensity (Years) & $\begin{array}{r}-0.001 \\
(0.008)\end{array}$ & $\begin{array}{r}-0.000 \\
(0.008)\end{array}$ & $\begin{array}{r}-0.001 \\
(0.008)\end{array}$ \\
\hline Exposure $x$ Rural & & $\begin{array}{r}0.160 * * * \\
(0.030)\end{array}$ & \\
\hline Exposure $\mathrm{x}$ Region 1 & & & $\begin{array}{r}0.550 * * * \\
(0.061)\end{array}$ \\
\hline Exposure $\mathrm{x}$ Region 2 & & & $\begin{array}{r}0.182 * * * \\
(0.062)\end{array}$ \\
\hline Exposure $x$ Region 3 & & & $\begin{array}{r}0.230 * * * \\
(0.050)\end{array}$ \\
\hline Exposure $x$ Region 5 & & & $\begin{array}{r}0.340 * * * \\
(0.060)\end{array}$ \\
\hline Exposure $x$ Region 6 & & & $\begin{array}{r}0.310 * * * \\
(0.060)\end{array}$ \\
\hline Constant & $\begin{array}{r}2.800 * * * \\
(0.090)\end{array}$ & $\begin{array}{r}2.821^{* * *} \\
(0.090)\end{array}$ & $\begin{array}{r}2.901^{* * *} \\
(0.091)\end{array}$ \\
\hline $\begin{array}{l}\text { Obs } \\
\text { R Squared }\end{array}$ & $\begin{array}{r}78,965 \\
0.343\end{array}$ & $\begin{array}{r}78,965 \\
0.344\end{array}$ & $\begin{array}{r}78,965 \\
0.344\end{array}$ \\
\hline
\end{tabular}


Table 5. 47 Spill Over Effect on Individual Education Expenditure (Children aged 6-15 living with beneficiary)

\begin{tabular}{|c|c|c|c|c|}
\hline & (1) & (2) & (3) & (4) \\
\hline Under Six & $\begin{array}{r}0.121^{* * *} \\
(0.020)\end{array}$ & $\begin{array}{r}0.110^{* * *} \\
(0.020)\end{array}$ & $\begin{array}{r}0.120 * * * \\
(0.020)\end{array}$ & $\begin{array}{r}0.110^{* * *} \\
(0.021)\end{array}$ \\
\hline Under Six x Post & $\begin{array}{r}0.000 \\
(0.010)\end{array}$ & & $\begin{array}{r}-0.004 \\
(0.020)\end{array}$ & $\begin{array}{r}-0.120 * * * \\
(0.030)\end{array}$ \\
\hline Under Six x Year2004 & & $\begin{array}{r}0.020 \\
(0.020)\end{array}$ & & \\
\hline Under Six x Year2006 & & $\begin{array}{r}-0.058 * * \\
(0.020)\end{array}$ & & \\
\hline Under Six x Year2008 & & $\begin{array}{r}0.032 \\
(0.020)\end{array}$ & & \\
\hline Under Six x Year2010 & & $\begin{array}{r}0.038 \\
(0.031)\end{array}$ & & \\
\hline Under Six x Year2012 & & $\begin{array}{r}-0.005 \\
(0.034)\end{array}$ & & \\
\hline Under Six x Year2014 & & $\begin{array}{r}0.029 \\
(0.032)\end{array}$ & & \\
\hline Under Six x Year2016 & & $\begin{array}{r}0.001 \\
(0.031)\end{array}$ & & \\
\hline Under Six x Post $x$ Rural & & & $\begin{array}{r}0.005 \\
(0.020)\end{array}$ & \\
\hline Under Six x Post $x$ Region 1 & & & & $\begin{array}{r}0.370 * * * \\
(0.030)\end{array}$ \\
\hline Under Six x Post $x$ Region 2 & & & & $\begin{array}{r}0.031 \\
(0.030)\end{array}$ \\
\hline Under Six $x$ Post $x$ Region 3 & & & & $\begin{array}{r}0.120 * * * \\
(0.030)\end{array}$ \\
\hline Under Six $x$ Post $x$ Region 5 & & & & $\begin{array}{r}0.160 * * * \\
(0.041)\end{array}$ \\
\hline Under Six x Post x Region 6 & & & & $\begin{array}{r}0.032 \\
(0.023)\end{array}$ \\
\hline Constant & $\begin{array}{r}4.311^{* * *} \\
(0.060)\end{array}$ & $\begin{array}{r}4.310 * * * \\
(0.060)\end{array}$ & $\begin{array}{r}4.310 * * * \\
(0.061)\end{array}$ & $\begin{array}{r}4.350 * * * \\
(0.061)\end{array}$ \\
\hline Obs & 71,076 & 71,076 & 71,076 & 71,076 \\
\hline R Squared & 0.638 & 0.638 & 0.638 & 0.640 \\
\hline
\end{tabular}


Table 5. 48 Persistent Effect on School Attendance (Children aged 6-15 with previously exposing to $\mathrm{FHI}$ )

\section{(1)}

(2)

Exposure $(\mathrm{Y} / \mathrm{N})$

$\begin{array}{rr}0.001 & -0.032 * * * \\ (0.005) & (0.006) \\ 0.002 * * & 0.002 * * \\ (0.001) & (0.001) \\ & 0.043 * * * \\ & (0.005)\end{array}$

Exposure $x$ Region 1

Exposure $x$ Region 2

Exposure $x$ Region 3

Exposure $x$ Region 5 $-0.009$ (0.010)

Exposure $\mathrm{x}$ Region 6

Constant

$0.750 * * *$

$0.760 * * *$

$0.750 * * *$

(0.010)

(0.010)

(0.010)

Obs 
Table 5. 49 Spill Over Effect on School Attendance (Children aged 6-15 living with beneficiary)

\begin{tabular}{|c|c|c|c|c|}
\hline & (1) & (2) & (3) & (4) \\
\hline Under Six & $\begin{array}{r}-0.002 \\
(0.007)\end{array}$ & $\begin{array}{r}-0.002 \\
(0.007)\end{array}$ & $\begin{array}{r}-0.001 \\
(0.007)\end{array}$ & $\begin{array}{r}-0.001 \\
(0.007)\end{array}$ \\
\hline Under Six x Post & $\begin{array}{r}0.028 * * * \\
(0.004)\end{array}$ & & $\begin{array}{r}0.013^{* *} \\
(0.005)\end{array}$ & $\begin{array}{r}0.043 * * * \\
(0.008)\end{array}$ \\
\hline Under Six x Year2004 & & $\begin{array}{r}-0.001 \\
(0.008)\end{array}$ & & \\
\hline Under Six x Year2006 & & $\begin{array}{r}0.009 \\
(0.008)\end{array}$ & & \\
\hline Under Six x Year2008 & & $\begin{array}{r}0.013 \\
(0.008)\end{array}$ & & \\
\hline Under Six x Year2010 & & $\begin{array}{r}0.041^{* * *} \\
(0.007)\end{array}$ & & \\
\hline Under Six x Year2012 & & $\begin{array}{r}0.042 * * * \\
(0.007)\end{array}$ & & \\
\hline Under Six x Year2014 & & $\begin{array}{r}0.031^{* * *} \\
(0.007)\end{array}$ & & \\
\hline Under Six x Year2016 & & $\begin{array}{r}0.037 * * * \\
(0.006)\end{array}$ & & \\
\hline Under Six x Post x Rural & & & $\begin{array}{r}0.019 * * * \\
(0.005)\end{array}$ & \\
\hline Under Six x Post $x$ Region 1 & & & & $\begin{array}{r}-0.032 * * * \\
(0.009)\end{array}$ \\
\hline Under Six x Post $x$ Region 2 & & & & $\begin{array}{r}-0.017^{*} \\
(0.010)\end{array}$ \\
\hline Under Six x Post $x$ Region 3 & & & & $\begin{array}{r}-0.020 * * \\
(0.009)\end{array}$ \\
\hline Under Six x Post $x$ Region 5 & & & & $\begin{array}{r}-0.021^{*} \\
(0.010)\end{array}$ \\
\hline Under Six x Post x Region 6 & & & & $\begin{array}{l}0.0057 \\
(0.010)\end{array}$ \\
\hline Constant & $\begin{array}{r}0.230 * * * \\
(0.020)\end{array}$ & $\begin{array}{r}0.230 * * * \\
(0.020)\end{array}$ & $\begin{array}{r}0.231^{* * *} \\
(0.020)\end{array}$ & $\begin{array}{r}0.220 * * * \\
(0.020)\end{array}$ \\
\hline Obs & 79,097 & 79,097 & 79,097 & 79,097 \\
\hline R Squared & 0.133 & 0.133 & 0.133 & 0.133 \\
\hline
\end{tabular}


Table 5. 50 Spill Over effect on Household Wage Income

\begin{tabular}{|c|c|c|c|c|}
\hline & $(1)$ & $(2)$ & (3) & (4) \\
\hline Under Six & $\begin{array}{r}0.100 * * * \\
(0.020)\end{array}$ & $\begin{array}{r}0.097^{* * *} \\
(0.020)\end{array}$ & $\begin{array}{r}0.100^{* * *} \\
(0.020)\end{array}$ & $\begin{array}{r}0.101 * * * \\
(0.020)\end{array}$ \\
\hline Under Six x Post & $\begin{array}{r}0.098 * * * \\
(0.021)\end{array}$ & & $\begin{array}{r}0.061^{* * *} \\
(0.021)\end{array}$ & $\begin{array}{r}0.110^{* * *} \\
(0.040)\end{array}$ \\
\hline Under Six x Year2004 & & $\begin{array}{r}0.023 \\
(0.030)\end{array}$ & & \\
\hline Under Six x Year2006 & & $\begin{array}{r}0.014 \\
(0.030)\end{array}$ & & \\
\hline Under Six x Year2008 & & $\begin{array}{r}0.043 \\
(0.030)\end{array}$ & & \\
\hline Under Six x Year2010 & & $\begin{array}{r}0.130 * * * \\
(0.030)\end{array}$ & & \\
\hline Under Six x Year2012 & & $\begin{array}{r}0.141^{* * *} \\
(0.030)\end{array}$ & & \\
\hline Under Six x Year2014 & & $\begin{array}{r}0.162^{* * *} \\
(0.030)\end{array}$ & & \\
\hline Under Six x Year2016 & & $\begin{array}{r}0.120 * * * \\
(0.030)\end{array}$ & & \\
\hline Under Six x Post x Rural & & & $\begin{array}{r}0.052 * * \\
(0.020)\end{array}$ & \\
\hline Under Six x Post x Region 1 & & & & $\begin{array}{r}0.210 * * * \\
(0.040)\end{array}$ \\
\hline Under Six x Post x Region 2 & & & & $\begin{array}{l}-0.034 \\
(0.042)\end{array}$ \\
\hline Under Six x Post $x$ Region 3 & & & & $\begin{array}{l}-0.037 \\
(0.044)\end{array}$ \\
\hline Under Six x Post $x$ Region 5 & & & & $\begin{array}{r}-0.072 * \\
(0.040)\end{array}$ \\
\hline Under Six x Post x Region 6 & & & & $\begin{array}{r}-0.170 * * * \\
(0.040)\end{array}$ \\
\hline Constant & $\begin{array}{r}6.940 * * * \\
(0.050)\end{array}$ & $\begin{array}{r}6.950 * * * \\
(0.051)\end{array}$ & $\begin{array}{r}6.940 * * * \\
(0.051)\end{array}$ & $\begin{array}{r}6.930 * * * \\
(0.060)\end{array}$ \\
\hline Obs & 58,700 & 58,700 & 58,700 & 58,700 \\
\hline R Squared & 0.559 & 0.559 & 0.559 & 0.560 \\
\hline
\end{tabular}


Table 5. 51 Spill Over effect on Household Income Per Capita

\begin{tabular}{|c|c|c|c|c|}
\hline & (1) & $(2)$ & (3) & (4) \\
\hline Under Six & $\begin{array}{r}0.022 * * \\
(0.011)\end{array}$ & $\begin{array}{l}0.020 * \\
(0.011)\end{array}$ & $\begin{array}{r}0.023 * * \\
(0.011)\end{array}$ & $\begin{array}{l}0.019 * \\
(0.010)\end{array}$ \\
\hline Under Six x Post & $\begin{array}{l}0.014^{*} \\
(0.009)\end{array}$ & & $\begin{array}{r}0.000 \\
(0.010)\end{array}$ & $\begin{array}{l}-0.022 \\
(0.020)\end{array}$ \\
\hline Under Six x Year2004 & & $\begin{array}{r}0.009 \\
(0.021)\end{array}$ & & \\
\hline Under Six x Year2006 & & $\begin{array}{r}0.023 \\
(0.020)\end{array}$ & & \\
\hline Under Six x Year2008 & & $\begin{array}{r}0.031^{* *} \\
(0.020)\end{array}$ & & \\
\hline Under Six x Year2010 & & $\begin{array}{r}-0.020 \\
(0.020)\end{array}$ & & \\
\hline Under Six x Year2012 & & $\begin{array}{r}0.017 \\
(0.020)\end{array}$ & & \\
\hline Under Six x Year2014 & & $\begin{array}{r}0.020 \\
(0.021)\end{array}$ & & \\
\hline Under Six x Year2016 & & $\begin{array}{r}0.031 * * \\
(0.022)\end{array}$ & & \\
\hline Under Six x Post x Rural & & & $\begin{array}{r}0.018 \\
(0.010)\end{array}$ & \\
\hline Under Six x Post x Region 1 & & & & $\begin{array}{r}0.180^{* * *} \\
(0.020)\end{array}$ \\
\hline Under Six x Post $x$ Region 2 & & & & $\begin{array}{c}-0.041^{*} \\
(0.020)\end{array}$ \\
\hline Under Six $x$ Post $x$ Region 3 & & & & $\begin{array}{r}0.060 * * * \\
(0.024)\end{array}$ \\
\hline Under Six $x$ Post $x$ Region 5 & & & & $\begin{array}{l}-0.013 \\
(0.020)\end{array}$ \\
\hline Under Six x Post $x$ Region 6 & & & & $\begin{array}{c}-0.002 \\
(0.020)\end{array}$ \\
\hline Constant & $\begin{array}{r}7.200 * * * \\
(0.030)\end{array}$ & $\begin{array}{r}7.200 * * * \\
(0.030)\end{array}$ & $\begin{array}{r}7.200 * * * \\
(0.030)\end{array}$ & $\begin{array}{r}7.210 * * * \\
(0.033)\end{array}$ \\
\hline Obs & 94,645 & 94,645 & 94,645 & 94,645 \\
\hline R Squared & 0.696 & 0.696 & 0.696 & 0.697 \\
\hline
\end{tabular}


Table 5. 52 Spill Over effect on Household Expenditure Per Capita

\begin{tabular}{|c|c|c|c|c|}
\hline & (1) & (2) & (3) & (4) \\
\hline Under Six & $\begin{array}{r}-0.006 \\
(0.009)\end{array}$ & $\begin{array}{r}-0.007 \\
(0.010)\end{array}$ & $\begin{array}{r}-0.006 \\
(0.009)\end{array}$ & $\begin{array}{l}-0.008 \\
(0.009)\end{array}$ \\
\hline Under Six x Post & $\begin{array}{c}-0.013^{*} \\
(0.007)\end{array}$ & & $\begin{array}{r}-0.009 \\
(0.010)\end{array}$ & $\begin{array}{r}-0.059 * * * \\
(0.021)\end{array}$ \\
\hline Under Six x Year2004 & & $\begin{array}{r}0.002 \\
(0.010)\end{array}$ & & \\
\hline Under Six x Year2006 & & $\begin{array}{r}0.002 \\
(0.013)\end{array}$ & & \\
\hline Under Six x Year2008 & & $\begin{array}{r}0.015 \\
(0.010)\end{array}$ & & \\
\hline Under Six x Year2010 & & $\begin{array}{c}-0.024^{*} \\
(0.010)\end{array}$ & & \\
\hline Under Six x Year2012 & & $\begin{array}{r}-0.012 \\
(0.010)\end{array}$ & & \\
\hline Under Six x Year2014 & & $\begin{array}{r}-0.008 \\
(0.014)\end{array}$ & & \\
\hline Under Six x Year2016 & & $\begin{array}{r}-0.042 * * * \\
(0.012)\end{array}$ & & \\
\hline Under Six x Post x Rural & & & $\begin{array}{r}-0.004 \\
(0.009)\end{array}$ & \\
\hline Under Six x Post $x$ Region 1 & & & & $\begin{array}{r}0.190 * * * \\
(0.020)\end{array}$ \\
\hline Under Six x Post $x$ Region 2 & & & & $\begin{array}{r}0.015 \\
(0.020)\end{array}$ \\
\hline Under Six x Post $x$ Region 3 & & & & $\begin{array}{r}0.051^{* * *} \\
(0.020)\end{array}$ \\
\hline Under Six x Post $x$ Region 5 & & & & $\begin{array}{r}0.027 \\
(0.020)\end{array}$ \\
\hline Under Six x Post $x$ Region 6 & & & & $\begin{array}{c}-0.029 * \\
(0.024)\end{array}$ \\
\hline Constant & $\begin{array}{r}7.312^{* * *} \\
(0.020)\end{array}$ & $\begin{array}{r}7.311^{* * *} \\
(0.020)\end{array}$ & $\begin{array}{r}7.311^{* * *} \\
(0.021)\end{array}$ & $\begin{array}{r}7.320 * * * \\
(0.021)\end{array}$ \\
\hline Obs & 94,527 & 94,527 & 94,527 & 94,527 \\
\hline R Squared & 0.795 & 0.795 & 0.795 & 0.796 \\
\hline
\end{tabular}


Table 5. 53 Spill Over Effect on Household Expenditure Per Adult Equivalent

\begin{tabular}{|c|c|c|c|c|}
\hline & (1) & $(2)$ & (3) & (4) \\
\hline Under Six & $\begin{array}{c}0.017^{*} \\
(0.009)\end{array}$ & $\begin{array}{l}0.016^{*} \\
(0.009)\end{array}$ & $\begin{array}{c}0.017^{*} \\
(0.009)\end{array}$ & $\begin{array}{l}0.016 * \\
(0.009)\end{array}$ \\
\hline Under Six x Post & $\begin{array}{r}-0.008 \\
(0.007)\end{array}$ & & $\begin{array}{r}-0.001 \\
(0.010)\end{array}$ & $\begin{array}{r}-0.053^{* * *} \\
(0.020)\end{array}$ \\
\hline Under Six x Year2004 & & $\begin{array}{r}0.003 \\
(0.011)\end{array}$ & & \\
\hline Under Six x Year2006 & & $\begin{array}{r}0.008 \\
(0.010)\end{array}$ & & \\
\hline Under Six x Year2008 & & $\begin{array}{r}0.017 \\
(0.010)\end{array}$ & & \\
\hline Under Six x Year2010 & & $\begin{array}{r}-0.019 \\
(0.010)\end{array}$ & & \\
\hline Under Six x Year2012 & & $\begin{array}{r}-0.006 \\
(0.014)\end{array}$ & & \\
\hline Under Six x Year2014 & & $\begin{array}{r}-0.005 \\
(0.014)\end{array}$ & & \\
\hline Under Six x Year2016 & & $\begin{array}{r}-0.036 * * * \\
(0.010)\end{array}$ & & \\
\hline Under Six x Post x Rural & & & $\begin{array}{r}-0.008 \\
(0.009)\end{array}$ & \\
\hline Under Six x Post x Region 1 & & & & $\begin{array}{r}0.180 * * * \\
(0.020)\end{array}$ \\
\hline Under Six x Post $x$ Region 2 & & & & $\begin{array}{r}0.006 \\
(0.022)\end{array}$ \\
\hline Under Six x Post $x$ Region 3 & & & & $\begin{array}{r}0.056 * * * \\
(0.022)\end{array}$ \\
\hline Under Six x Post $x$ Region 5 & & & & $\begin{array}{r}0.026 \\
(0.021)\end{array}$ \\
\hline Under Six x Post x Region 6 & & & & $\begin{array}{c}-0.029 * \\
(0.021)\end{array}$ \\
\hline Constant & $\begin{array}{r}7.660 * * * \\
(0.020)\end{array}$ & $\begin{array}{r}7.660 * * * \\
(0.020)\end{array}$ & $\begin{array}{r}7.661^{* * * *} \\
(0.020)\end{array}$ & $\begin{array}{r}7.670 * * * \\
(0.020)\end{array}$ \\
\hline Obs & 94,527 & 94,527 & 94,527 & 94,527 \\
\hline R Squared & 0.794 & 0.794 & 0.794 & 0.795 \\
\hline
\end{tabular}


Table 5. 54 Spill Over Effect on Household Education Expenditure

\begin{tabular}{|c|c|c|c|c|}
\hline & (1) & (2) & (3) & (4) \\
\hline Under Six & $\begin{array}{r}-0.191 * * * \\
(0.020)\end{array}$ & $\begin{array}{r}-0.191 * * * \\
(0.020)\end{array}$ & $\begin{array}{r}-0.191 * * * \\
(0.020)\end{array}$ & $\begin{array}{r}-0.191 * * * \\
(0.020)\end{array}$ \\
\hline Under Six x Post & $\begin{array}{r}-0.100 * * * \\
(0.020)\end{array}$ & & $\begin{array}{r}-0.120 * * * \\
(0.020)\end{array}$ & $\begin{array}{r}-0.170 * * * \\
(0.040)\end{array}$ \\
\hline Under Six x Year2004 & & $\begin{array}{r}0.011 \\
(0.030)\end{array}$ & & \\
\hline Under Six x Year2006 & & $\begin{array}{l}-0.042 \\
(0.031)\end{array}$ & & \\
\hline Under Six x Year2008 & & $\begin{array}{r}-0.037 \\
(0.030)\end{array}$ & & \\
\hline Under Six x Year2010 & & $\begin{array}{r}-0.085^{* * *} \\
(0.030)\end{array}$ & & \\
\hline Under Six x Year2012 & & $\begin{array}{r}-0.130 * * * \\
(0.032)\end{array}$ & & \\
\hline Under Six x Year2014 & & $\begin{array}{r}-0.130 * * * \\
(0.030)\end{array}$ & & \\
\hline Under Six x Year2016 & & $\begin{array}{r}-0.160 * * * \\
(0.031)\end{array}$ & & \\
\hline Under Six x Post x Rural & & & $\begin{array}{r}0.031 \\
(0.022)\end{array}$ & \\
\hline Under Six x Post x Region 1 & & & & $\begin{array}{r}0.202 * * * \\
(0.040)\end{array}$ \\
\hline Under Six x Post x Region 2 & & & & $\begin{array}{r}0.003 \\
(0.041)\end{array}$ \\
\hline Under Six x Post x Region 3 & & & & $\begin{array}{l}0.076^{*} \\
(0.041)\end{array}$ \\
\hline Under Six x Post x Region 5 & & & & $\begin{array}{r}0.140 * * * \\
(0.050)\end{array}$ \\
\hline Under Six x Post x Region 6 & & & & $\begin{array}{l}-0.000 \\
(0.040)\end{array}$ \\
\hline Constant & $\begin{array}{r}2.260 * * * \\
(0.070)\end{array}$ & $\begin{array}{r}2.250 * * * \\
(0.070)\end{array}$ & $\begin{array}{r}2.260 * * * \\
(0.071)\end{array}$ & $\begin{array}{r}2.280 * * * \\
(0.071)\end{array}$ \\
\hline Obs & 61,716 & 61,716 & 61,716 & 61,716 \\
\hline R Squared & 0.558 & 0.558 & 0.558 & 0.559 \\
\hline
\end{tabular}


Table 5. 55 Spill Over Effect on Household Healthcare Expenditure

\begin{tabular}{|c|c|c|c|c|}
\hline & (1) & (2) & (3) & (4) \\
\hline Under Six & $\begin{array}{r}-0.048 \\
(0.040)\end{array}$ & $\begin{array}{r}-0.047 \\
(0.040)\end{array}$ & $\begin{array}{r}-0.049 \\
(0.040)\end{array}$ & $\begin{array}{r}-0.050 \\
(0.040)\end{array}$ \\
\hline Under Six x Post & $\begin{array}{r}0.044 \\
(0.030)\end{array}$ & & $\begin{array}{r}0.079 * * \\
(0.040)\end{array}$ & $\begin{array}{c}-0.13^{* *} \\
(0.060)\end{array}$ \\
\hline Under Six x Year2004 & & $\begin{array}{r}-0.004 \\
(0.050)\end{array}$ & & \\
\hline Under Six x Year2006 & & $\begin{array}{r}0.035 \\
(0.050)\end{array}$ & & \\
\hline Under Six x Year2008 & & $\begin{array}{r}0.070 \\
(0.051)\end{array}$ & & \\
\hline Under Six x Year2010 & & $\begin{array}{r}0.067 \\
(0.051)\end{array}$ & & \\
\hline Under Six x Year2012 & & $\begin{array}{r}0.066 \\
(0.050)\end{array}$ & & \\
\hline Under Six x Year2014 & & $\begin{array}{r}0.045 \\
(0.052)\end{array}$ & & \\
\hline Under Six x Year2016 & & $\begin{array}{r}-0.030 \\
(0.051)\end{array}$ & & \\
\hline Under Six x Post x Rural & & & $\begin{array}{r}-0.049 \\
(0.031)\end{array}$ & \\
\hline Under Six x Post $x$ Region 1 & & & & $\begin{array}{r}0.282 * * * \\
(0.060)\end{array}$ \\
\hline Under Six x Post x Region 2 & & & & $\begin{array}{r}0.240 * * * \\
(0.062)\end{array}$ \\
\hline Under Six x Post $x$ Region 3 & & & & $\begin{array}{l}0.112 * \\
(0.060)\end{array}$ \\
\hline Under Six x Post x Region 5 & & & & $\begin{array}{r}0.190 * * * \\
(0.070)\end{array}$ \\
\hline Under Six x Post x Region 6 & & & & $\begin{array}{r}0.150 * * \\
(0.064)\end{array}$ \\
\hline Constant & $\begin{array}{r}3.850 * * * \\
(0.091)\end{array}$ & $\begin{array}{r}3.840 * * * \\
(0.092)\end{array}$ & $\begin{array}{r}3.840 * * * \\
(0.090)\end{array}$ & $\begin{array}{r}3.901 * * * \\
(0.090)\end{array}$ \\
\hline Obs & 60,792 & 60,792 & 60,792 & 60,792 \\
\hline R Squared & 0.194 & 0.194 & 0.194 & 0.194 \\
\hline
\end{tabular}


Table 5. 56 Spill Over Effect on Catastrophic Health Expenditure ( $>10 \%$ of household consumption)

\begin{tabular}{|c|c|c|c|c|}
\hline & $(1)$ & $(2)$ & (3) & (4) \\
\hline Under Six & $\begin{array}{r}0.069 * * * \\
(0.009)\end{array}$ & $\begin{array}{r}0.070 * * * \\
(0.009)\end{array}$ & $\begin{array}{r}0.068 * * * \\
(0.009)\end{array}$ & $\begin{array}{r}0.067 * * * \\
(0.009)\end{array}$ \\
\hline Under Six x Post & $\begin{array}{r}0.004 \\
(0.007)\end{array}$ & & $\begin{array}{r}0.033 * * * \\
(0.010)\end{array}$ & $\begin{array}{l}-0.004 \\
(0.020)\end{array}$ \\
\hline Under Six x Year2004 & & $\begin{array}{c}-0.002 \\
(0.010)\end{array}$ & & \\
\hline Under Six x Year2006 & & $\begin{array}{c}-0.021^{*} \\
(0.010)\end{array}$ & & \\
\hline Under Six x Year2008 & & $\begin{array}{r}0.017 \\
(0.010)\end{array}$ & & \\
\hline Under Six x Year2010 & & $\begin{array}{l}-0.006 \\
(0.012)\end{array}$ & & \\
\hline Under Six x Year2012 & & $\begin{array}{l}-0.005 \\
(0.013)\end{array}$ & & \\
\hline Under Six x Year2014 & & $\begin{array}{r}0.031^{* *} \\
(0.010)\end{array}$ & & \\
\hline Under Six x Year2016 & & $\begin{array}{r}0.006 \\
(0.012)\end{array}$ & & \\
\hline Under Six x Post x Rural & & & $\begin{array}{r}-0.039 * * * \\
(0.009)\end{array}$ & \\
\hline Under Six x Post x Region 1 & & & & $\begin{array}{r}0.026 \\
(0.020)\end{array}$ \\
\hline Under Six x Post x Region 2 & & & & $\begin{array}{r}-0.040 * * \\
(0.020)\end{array}$ \\
\hline Under Six x Post x Region 3 & & & & $\begin{array}{r}0.003 \\
(0.021)\end{array}$ \\
\hline Under Six x Post x Region 5 & & & & $\begin{array}{r}0.049 * * * \\
(0.021)\end{array}$ \\
\hline Under Six x Post x Region 6 & & & & $\begin{array}{r}0.034 * * \\
(0.021)\end{array}$ \\
\hline Constant & $\begin{array}{r}0.312^{* * *} \\
(0.020)\end{array}$ & $\begin{array}{r}0.312 * * * \\
(0.020)\end{array}$ & $\begin{array}{r}0.303 * * * \\
(0.020)\end{array}$ & $\begin{array}{r}0.322 * * * \\
(0.020)\end{array}$ \\
\hline Obs & 94,527 & 94,527 & 94,527 & 94,527 \\
\hline R Squared & 0.076 & 0.076 & 0.076 & 0.076 \\
\hline
\end{tabular}


Table 5. 57 Spill Over Effect on Catastrophic Health Expenditure ( $>25 \%$ of household consumption)

\begin{tabular}{|c|c|c|c|c|}
\hline & (1) & (2) & (3) & (4) \\
\hline Under Six & $\begin{array}{r}0.070 * * * \\
(0.009)\end{array}$ & $\begin{array}{r}0.071^{* * *} \\
(0.009)\end{array}$ & $\begin{array}{r}0.069 * * * \\
(0.009)\end{array}$ & $\begin{array}{r}0.067 * * * \\
(0.009)\end{array}$ \\
\hline Under Six x Post & $\begin{array}{r}0.004 \\
(0.007)\end{array}$ & & $\begin{array}{r}0.034^{* * *} \\
(0.010)\end{array}$ & $\begin{array}{r}-0.005 \\
(0.021)\end{array}$ \\
\hline Under Six x Year2004 & & $\begin{array}{r}-0.001 \\
(0.011)\end{array}$ & & \\
\hline Under Six x Year2006 & & $\begin{array}{r}-0.020 \\
(0.010)\end{array}$ & & \\
\hline Under Six x Year2008 & & $\begin{array}{r}0.018 \\
(0.010)\end{array}$ & & \\
\hline Under Six x Year2010 & & $\begin{array}{l}-0.005 \\
(0.013)\end{array}$ & & \\
\hline Under Six x Year2012 & & $\begin{array}{r}-0.005 \\
(0.012)\end{array}$ & & \\
\hline Under Six x Year2014 & & $\begin{array}{r}0.031 * * * \\
(0.010)\end{array}$ & & \\
\hline Under Six x Year2016 & & $\begin{array}{r}0.005 \\
(0.013)\end{array}$ & & \\
\hline Under Six $x$ Post $x$ Rural & & & $\begin{array}{r}-0.039 * * * \\
(0.009)\end{array}$ & \\
\hline Under Six x Post $x$ Region 1 & & & & $\begin{array}{r}0.026 \\
(0.022)\end{array}$ \\
\hline Under Six x Post x Region 2 & & & & $\begin{array}{r}-0.038 * * \\
(0.022)\end{array}$ \\
\hline Under Six x Post $x$ Region 3 & & & & $\begin{array}{r}0.004 \\
(0.021)\end{array}$ \\
\hline Under Six $x$ Post $\times$ Region 5 & & & & $\begin{array}{r}0.050 * * * \\
(0.020)\end{array}$ \\
\hline Under Six $x$ Post $x$ Region 6 & & & & $\begin{array}{r}0.035^{* *} \\
(0.020)\end{array}$ \\
\hline Constant & $\begin{array}{r}0.310 * * * \\
(0.020)\end{array}$ & $\begin{array}{r}0.310 * * * \\
(0.020)\end{array}$ & $\begin{array}{r}0.310 * * * \\
(0.020)\end{array}$ & $\begin{array}{r}0.320 * * * \\
(0.020)\end{array}$ \\
\hline Obs & 94,527 & 94,527 & 94,527 & 94,527 \\
\hline R Squared & 0.075 & 0.075 & 0.075 & 0.075 \\
\hline
\end{tabular}


Table 5. 58 Spill Over Effect on Catastrophic Health Expenditure ( $>40 \%$ of household consumption)

\begin{tabular}{|c|c|c|c|c|}
\hline & (1) & (2) & (3) & (4) \\
\hline Under Six & $\begin{array}{r}0.072 * * * \\
(0.009)\end{array}$ & $\begin{array}{r}0.072^{* * *} \\
(0.009)\end{array}$ & $\begin{array}{r}0.071 * * * \\
(0.009)\end{array}$ & $\begin{array}{r}0.069 * * * \\
(0.009)\end{array}$ \\
\hline Under Six x Post & $\begin{array}{r}0.004 \\
(0.007)\end{array}$ & & $\begin{array}{r}0.035 * * * \\
(0.010)\end{array}$ & $\begin{array}{l}-0.007 \\
(0.021)\end{array}$ \\
\hline Under Six x Year2004 & & $\begin{array}{r}-0.000 \\
(0.010)\end{array}$ & & \\
\hline Under Six x Year2006 & & $\begin{array}{r}-0.020 \\
(0.010)\end{array}$ & & \\
\hline Under Six x Year2008 & & $\begin{array}{r}0.018 \\
(0.012)\end{array}$ & & \\
\hline Under Six x Year2010 & & $\begin{array}{r}-0.006 \\
(0.013)\end{array}$ & & \\
\hline Under Six x Year2012 & & $\begin{array}{l}-0.005 \\
(0.012)\end{array}$ & & \\
\hline Under Six x Year2014 & & $\begin{array}{r}0.031 * * \\
(0.011)\end{array}$ & & \\
\hline Under Six x Year2016 & & $\begin{array}{r}0.005 \\
(0.014)\end{array}$ & & \\
\hline Under Six x Post x Rural & & & $\begin{array}{r}-0.042 * * * \\
(0.009)\end{array}$ & \\
\hline Under Six x Post x Region 1 & & & & $\begin{array}{r}0.028 \\
(0.020)\end{array}$ \\
\hline Under Six x Post x Region 2 & & & & $\begin{array}{r}-0.038^{* *} \\
(0.020)\end{array}$ \\
\hline Under Six x Post x Region 3 & & & & $\begin{array}{r}0.005 \\
(0.022)\end{array}$ \\
\hline Under Six x Post $x$ Region 5 & & & & $\begin{array}{r}0.052 * * * \\
(0.022)\end{array}$ \\
\hline Under Six x Post x Region 6 & & & & $\begin{array}{r}0.037 * * \\
(0.021)\end{array}$ \\
\hline Constant & $\begin{array}{r}0.320 * * * \\
(0.020)\end{array}$ & $\begin{array}{r}0.320 * * * \\
(0.020)\end{array}$ & $\begin{array}{r}0.310 * * * \\
(0.020)\end{array}$ & $\begin{array}{r}0.330 * * * \\
(0.020)\end{array}$ \\
\hline Obs & 94,527 & 94,527 & 94,527 & 94,527 \\
\hline R Squared & 0.073 & 0.073 & 0.073 & 0.074 \\
\hline
\end{tabular}


Table 5. 59 Spill Over Effect on Household Health Insurance Coverage

\begin{tabular}{|c|c|c|c|c|}
\hline & (1) & (2) & (3) & (4) \\
\hline Under Six & $\begin{array}{r}-0.110 * * * \\
(0.010)\end{array}$ & $\begin{array}{r}-0.110^{* * *} \\
(0.010)\end{array}$ & $\begin{array}{r}-0.110 * * * \\
(0.010)\end{array}$ & $\begin{array}{r}-0.110 * * * \\
(0.010)\end{array}$ \\
\hline Under Six x Post & $\begin{array}{r}0.121^{* * * *} \\
(0.008)\end{array}$ & & $\begin{array}{r}0.110 * * * \\
(0.010)\end{array}$ & $\begin{array}{r}0.160 * * * \\
(0.010)\end{array}$ \\
\hline Under Six x Year2006 & & $\begin{array}{r}0.140 * * * \\
(0.010)\end{array}$ & & \\
\hline Under Six x Year2008 & & $\begin{array}{r}0.140 * * * \\
(0.010)\end{array}$ & & \\
\hline Under Six x Year2010 & & $\begin{array}{r}0.140 * * * \\
(0.010)\end{array}$ & & \\
\hline Under Six x Year2012 & & $\begin{array}{r}0.130 * * * \\
(0.010)\end{array}$ & & \\
\hline Under Six x Year2014 & & $\begin{array}{r}0.110 * * * \\
(0.010)\end{array}$ & & \\
\hline Under Six x Year2016 & & $\begin{array}{r}0.093 * * * \\
(0.010)\end{array}$ & & \\
\hline Under Six x Post $x$ Rural & & & $\begin{array}{r}0.019 * * * \\
(0.007)\end{array}$ & \\
\hline Under Six x Post $x$ Region 1 & & & & $\begin{array}{r}-0.041 * * * \\
(0.010)\end{array}$ \\
\hline Under Six x Post $x$ Region 2 & & & & $\begin{array}{r}-0.027 * * \\
(0.011)\end{array}$ \\
\hline Under Six $x$ Post $x$ Region 3 & & & & $\begin{array}{r}-0.046 * * * \\
(0.010)\end{array}$ \\
\hline Under Six x Post $x$ Region 5 & & & & $\begin{array}{l}-0.023 \\
(0.010)\end{array}$ \\
\hline Under Six x Post x Region 6 & & & & $\begin{array}{r}-0.038^{* * *} \\
(0.010)\end{array}$ \\
\hline Constant & $\begin{array}{r}0.770 * * * \\
(0.020)\end{array}$ & $\begin{array}{r}0.760 * * * \\
(0.020)\end{array}$ & $\begin{array}{r}0.770 * * * \\
(0.020)\end{array}$ & $\begin{array}{r}0.750 * * * \\
(0.020)\end{array}$ \\
\hline Obs & 65,164 & 65,164 & 65,164 & 65,164 \\
\hline R Squared & 0.204 & 0.205 & 0.204 & 0.205 \\
\hline
\end{tabular}


Table 5. 60 Spill Over Effect on Caloric Consumption Per Adult Equivalent

\begin{tabular}{lr}
\hline & $(1)$ \\
\hline Under Six & -26.2 \\
& $(16.4)$ \\
Under Six x Post & $-87.9 * * *$
\end{tabular}

(1) (2)

(3)

$-30.7^{*}$

$-28.1^{*}$

(17.3)

(16.4)

$-27.2^{*}$

$(16.4)$

(12.3)

$-43.4^{* *}$

(16.4)

(17.4)

Under Six x Year2004

17.7

(22.5)

Under Six x Year2006

$-40.4^{*}$

(22.8)

Under Six x Year2008

$-49.4 * *$

(22.5)

Under Six x Year2010

$-94.5^{* * *}$

(21.7)

Under Six x Year2012

$-96.3 * * *$

(21.9)

Under Six x Year2014

$-103.7^{* * *}$

(22.0)

Under Six x Year2016

$-112.6 * * *$

(22.0)

Under Six x Post x Rural

$-60.1 * * *$

(16.6)

Under Six x Post x Region 1

$53.5^{*}$

Under Six $x$ Post $x$ Region 2

Under Six x Post $x$ Region 3

$61.3^{* *}$

Under Six $x$ Post $x$ Region 5

Under Six x Post $x$ Region 6

(30.7)

Constant

2190.6***

2189.4***

2186.5***

2197.3***

(39.0)

(39.1)

(39.1)

(39.7)

Obs

94,691

94,691

94,691

94,691

R Squared

0.085

0.085

0.085

0.085 


\section{References}

Abrokwah, S. O., Moser, C. M., \& Norton, E. C. (2014). The effect of social health insurance on prenatal care: the case of Ghana. International journal of health care finance economics, 14(4), 385-406. doi:https://doi.org/10.1007/s10754-014-9155-8

Adams, K. P., Lybbert, T. J., Vosti, S. A., Ayifah, E., Arimond, M., Adu-Afarwuah, S., \& Dewey, K. G. (2018). Unintended effects of a targeted maternal and child nutrition intervention on household expenditures, labor income, and the nutritional status of non-targeted siblings in Ghana. World Development, 107, 138-150. doi:https://doi.org/10.1016/j.worlddev.2018.02.025

Adhvaryu, A., \& Nyshadham, A. (2016). Endowments at birth and parents' investments in children. The Economic Journal, 126(593), 781-820. doi:https://doi.org/10.1111/ecoj.12186

Adler, N. E., Boyce, T., Chesney, M. A., Cohen, S., Folkman, S., Kahn, R. L., \& Syme, S. L. (1994). Socioeconomic status and health: The challenge of the gradient. American Psychologist, 49(1), 15-24. doi:https://doi.org/10.1037/0003-066X.49.1.15

Alkenbrack, S., \& Lindelow, M. (2015). The impact of community - based health insurance on utilization and out - of - pocket expenditures in Lao People's Democratic Republic. Health Economics, 24(4), 379-399. doi:https://doi.org/10.1002/hec.3023

Andersen, R. M. (1995). Revisiting the behavioral model and access to medical care: Does it matter? Journal of Health and Social Behavior, 36(1), 1-10. doi:10.2307/2137284

Angrist, J. D., \& Pischke, J.-S. (2008). Mostly harmless econometrics: An empiricist's companion. Princeton, New Jersey: Princeton university press.

Aron-Dine, A., Einav, L., \& Finkelstein, A. (2013). The RAND Health Insurance Experiment, three decades later. Journal of Economic Perspectives, 27(1), 197-222. doi:http://dx.doi.org/10.1257/jep.27.1.197

Arrow, K. J. (1963). Uncertainty and the welfare economics of medical care. The American Economic Review, 53(5), 941-973. Retrieved from www.jstor.org/stable/1812044

Aryeetey, G. C., Westeneng, J., Spaan, E., Jehu-Appiah, C., Agyepong, I. A., \& Baltussen, R. (2016). Can health insurance protect against out-of-pocket and catastrophic expenditures and also support poverty reduction? Evidence from Ghana's National Health Insurance Scheme. International Journal for Equity in Health, 15(1), 116. doi:10.1186/s12939-016-0401-1

Ashraf, N. (2009). Spousal control and intra-household decision making: An experimental study in the Philippines. American Economic Review, 99(4), 1245-1277. doi:10.1257/aer.99.4.1245

Austin, P. C. (2011). An introduction to Propensity Score Methods for reducing the effects of confounding in observational studies. Multivariate Behavioral Research, 46(3), 399424. doi:10.1080/00273171.2011.568786

Baeza, C. C., \& Packard, T. G. (2006). Beyond survival: protecting households from health shocks in Latin America (English). Washington DC: The World Bank.

Barrington, D. S., \& James, S. A. (2017). Receipt of public assistance during childhood and hypertension risk in adulthood. Annals of epidemiology, 27(2), 108-114. e102. doi:10.1016/j.annepidem.2016.11.012 
Beuermann, D., \& Garzon, C. P. (2016). Healthy to work: the impact of free public healthcare on health status and labor supply in Jamaica. IDB Working Paper Series.

Blanchet, N. J., Fink, G., \& Osei-Akoto, I. (2012). The effect of Ghana's National Health Insurance Scheme on health care utilisation. Ghana medical journal, 46(2), 76-84. Retrieved from https://www.ncbi.nlm.nih.gov/pmc/articles/PMC3426378/

Brook, R. H., Keeler, E. B., Lohr, K. N., Newhouse, J. P., Ware, J. E., Rogers, W. H., . . Camp, P. (2006). The health insurance experiment: A Classic RAND study speaks to the current health care reform debate. RAND Corporation. Retrieved from https://www.rand.org/pubs/research_briefs/RB9174.html

Brook, R. H., Ware, J. E., Rogers, W. H., Keeler, E. B., Davies, A. R., Sherbourne, C. D., . . Newhouse, J. P. (1984). The effect of coinsurance on the health of adults. RAND Corporation. Retrieved from https://www.rand.org/pubs/reports/R3055.html

Busse, R., Blümel, M., Knieps, F., \& Bärnighausen, T. (2017). Statutory health insurance in Germany: a health system shaped by 135 years of solidarity, self-governance, and competition. The Lancet 390(10097), 882-897. doi:https://doi.org/10.1016/S01406736(17)31280-1

Camacho, A., \& Conover, E. (2013). Effects of subsidized health insurance on newborn health in a developing country. Economic Development and Cultural Change, 61(3), 633-658. doi:10.1086/669263

Campbell, D. T., \& Stanley, J. C. (2015). Experimental and quasi-experimental designs for research: Ravenio Books.

Carrin, G., Waelkens, M.-P., \& Criel, B. (2005). Community-based health insurance in developing countries: a study of its contribution to the performance of health financing systems. Tropical Medicine \& International Health, 10(8), 799-811. doi:10.1111/j.1365-3156.2005.01455.x

Case, A., Fertig, A., \& Paxson, C. (2005). The lasting impact of childhood health and circumstance. Journal of health economics, 24(2), 365-389. doi:https://doi.org/10.1016/j.jhealeco.2004.09.008

Case, A., Lubotsky, D., \& Paxson, C. (2002). Economic status and health in childhood: The origins of the gradient. American Economic Review, 92(5), 1308-1334. doi:10.1257/000282802762024520

Case, A., \& Paxson, C. (2008). Stature and status: Height, ability, and labor market outcomes. Journal of Political Economy, 116(3), 499-532. doi:10.1086/589524

Celhay, P., Martinez, S., Muñoz, M., Perez, M., \& Perez-Cuevas, R. (2019). Long-term effects of public health insurance on the health of children in Mexico: a retrospective study. The Lancet Global Health, 7(10), e1448-e1457. doi:https://doi.org/10.1016/S2214$109 \times(19) 30326-2$

Chen, Y., \& Jin, G. Z. (2012). Does health insurance coverage lead to better health and educational outcomes? Evidence from rural China. Journal of Health Economics, 31(1), 1-14. doi:https://doi.org/10.1016/j.jhealeco.2011.11.001

Cohodes, S. R., Grossman, D. S., Kleiner, S. A., \& Lovenheim, M. F. (2016). The effect of child health insurance access on schooling: Evidence from public insurance expansions. Journal of Human Resources, University of Wisconsin Press, 51(3), 727-759. Retrieved from https://ideas.repec.org/a/uwp/jhriss/v51y2016i3p727-759.html

Currie, J. (2009). Healthy, wealthy, and wise: Socioeconomic status, poor health in childhood, and human capital development. Journal of Economic Literature, 47(1), 87-122. doi:10.1257/jel.47.1.87 
Currie, J., \& Hyson, R. (1999). Is the impact of health shocks cushioned by socioeconomic status? The case of low birthweight. The American Economic Review, 89(2), 245-250. Retrieved from www.jstor.org/stable/117114

Currie, J., \& Madrian, B. C. (1999). Chapter 50 Health, health insurance and the labor market. In Handbook of Labor Economics (Vol. 3, pp. 3309-3416): Elsevier.

Dao, H. L., Somanathan, A., Tandon, A., Hurt, K. L., \& Fuenzalida-Puelma, H. L. (2014). Moving toward Universal Coverage of Social Health Insurance in Vietnam: Assessment and Options. Washington DC: The World Bank.

Dao, H. T., Waters, H., \& Le, Q. V. (2008). User fees and health service utilization in Vietnam: How to protect the poor? Public Health, 122(10), 1068-1078. doi:https://doi.org/10.1016/j.puhe.2008.01.002

de Meza, D. (1983). Health insurance and the demand for medical care. Journal of Health Economics, 2(1), 47-54. doi:https://doi.org/10.1016/0167-6296(83)90011-5

Deaton, A. (2013). The great escape: Health, wealth, and the origins of inequality. Princeton, New Jersey: Princeton University Press.

Deaton, A., \& Zaidi, S. (2002). Guidelines for constructing consumption aggregates for welfare analysis (Vol. 135). Washington DC: World Bank Publications.

Duflo, E. (2003). Grandmothers and granddaughters: old - age pensions and intrahousehold allocation in South Africa. The World Bank Economic Review, 17(1), 1-25. Retrieved from https://www.jstor.org/stable/3990043

Ekman, B. (2004). Community-based health insurance in low-income countries: a systematic review of the evidence. Health Policy and Planning, 19(5), 249-270. doi:10.1093/heapol/czh031

Erlangga, D., Suhrcke, M., Ali, S., \& Bloor, K. (2019). The impact of public health insurance on health care utilisation, financial protection and health status in low- and middleincome countries: A systematic review. PLOS ONE, 14(8), e0219731. doi:10.1371/journal.pone.0219731

Feldstein, M. S. (1971). Hospital cost inflation: A study of nonprofit price dynamics. The American Economic Review, 61(5), 853-872. Retrieved from www.jstor.org/stable/1813146

Feldstein, M. S. (1973). The welfare loss of excess health insurance. Journal of Political Economy, 81(2, Part 1), 251-280. doi:10.1086/260027

Fenny, A. P., Asante, F. A., Enemark, U., \& Hansen, K. S. (2014). Treatment-seeking behaviour and social health insurance in Africa: the case of Ghana under the National Health Insurance Scheme. Global journal of health science, 7(1), 296-314. doi:10.5539/gjhs.v7n1p296

Fink, G., Robyn, P. J., Sié, A., \& Sauerborn, R. (2013). Does health insurance improve health?: Evidence from a randomized community-based insurance rollout in rural Burkina Faso. Journal of Health Economics, 32(6), 1043-1056. doi:https://doi.org/10.1016/j.jhealeco.2013.08.003

Finkelstein, A., Taubman, S., Wright, B., Bernstein, M., Gruber, J., Newhouse, J. P., . . . Group, O. H. S. (2012). The Oregon Health Insurance Experiment: Evidence from the First Year*. The Quarterly Journal of Economics, 127(3), 1057-1106. doi:10.1093/qje/qjs020

Fisher, E. S. (2003). Medical care - Is more always better? New England Journal of Medicine, 349(17), 1665-1667. doi:10.1056/NEJMe038149 
Fitzpatrick, A., \& Thornton, R. (2018). The effects of health insurance within families: Experimental evidence from Nicaragua. The World Bank Economic Review, 33(3), 736-749. doi:10.1093/wber/lhx012

Fitzsimons, E., Malde, B., Mesnard, A., \& Vera-Hernández, M. (2016). Nutrition, information and household behavior: Experimental evidence from Malawi. Journal of Development Economics, 122, 113-126. doi:https://doi.org/10.1016/j.jdeveco.2016.05.002

Galárraga, O., Sosa-Rubí, S. G., Salinas-Rodríguez, A., \& Sesma-Vázquez, S. (2010). Health insurance for the poor: impact on catastrophic and out-of-pocket health expenditures in Mexico. The European Journal of Health Economics, 11(5), 437-447. doi:10.1007/s10198-009-0180-3

Ghislandi, S., Manachotphong, W., \& Perego, V. M. E. (2015). The impact of Universal Health Coverage on health care consumption and risky behaviours: evidence from Thailand. Health Economics, Policy and Law, 10(3), 251-266. doi:10.1017/S1744133114000334

Giang, T. L. (2008). Social Health Insurance in Vietnam. MPRA Paper 9926, University Library of Munich, Germany. Retrieved from https://ideas.repec.org/p/pra/mprapa/9926.html

Giedion, U., Andrés Alfonso, E., \& Díaz, Y. (2013). The impact of universal coverage schemes in the developing world: A review of the existing evidence. UNICO Studies Series 25. World Bank, Washington DC. Retrieved from http://hdl.handle.net/10986/13302

Giedion, U., \& Villar Uribe, M. (2009). Colombia's universal health insurance system. Health Affairs, 28(3), 853-863. doi:10.1377/hlthaff.28.3.853

Glewwe, P., Gragnolati, M., \& Zaman, H. (2000). Who gained from Vietnam's boom in the 1990s?: an analysis of poverty and inequality trends (English). Washington DC: World Bank, Development Research Group, Poverty and Human Resources.

Grogger, J., Arnold, T., León, A. S., \& Ome, A. (2014). Heterogeneity in the effect of public health insurance on catastrophic out-of-pocket health expenditures: the case of Mexico. Health Policy and Planning, 30(5), 593-599. doi:10.1093/heapol/czu037

Guindon, G. E. (2014). The impact of health insurance on health services utilization and health outcomes in Vietnam. Health Economics, Policy and Law, 9(4), 359-382. doi:10.1017/S174413311400005X

Gustafsson-Wright, E., Janssens, W., \& van der Gaag, J. (2010). The inequitable impact of health shocks on the uninsured in Namibia. Health Policy and Planning, 26(2), 142156. doi:10.1093/heapol/czq029

Gustavo J. Bobonis. (2009). Is the allocation of resources within the household efficient? New evidence from a randomized experiment. Journal of Political Economy, 117(3), 453-503. doi:10.1086/600076

Haddad, L. J., \& Bouis, H. E. (1991). The impact of nutritional status on agricultural productivity: Wage evidence from the Philippines*. Oxford Bulletin of Economics and Statistics, 53(1), 45-68. doi:10.1111/j.1468-0084.1991.mp53001004.x

Hansen, H., \& Le, T. D. (2013). The importance of being surveyed: the representativeness and impact of the Vietnam household living standards surveys. Retrieved from https://www.econ.ku.dk/cam/calendar/seminars/cam01102013/VHLSS_Impacts_V7. pdf

Hanvoravongchai, P., \& Hsiao, W. C. (2007). Thailand: Achieving universal coverage with social health insurance. In Social Health Insurance for Developing Nations edited by W. C. Shaw. Washington DC: The World Bank. 
Hsiao, W., \& Shaw, R. P. (2007). Social health insurance for developing nations. Washington DC: The World Bank.

Jackson, M. I. (2015). Early childhood WIC participation, cognitive development and academic achievement. Social Science \& Medicine, 126, 145-153. doi:https://doi.org/10.1016/j.socscimed.2014.12.018

Johnson, R. C., \& Schoeni, R. F. (2011). Early-life origins of adult disease: national longitudinal population-based study of the United States. American journal of public health, 101(12), 2317-2324. doi:10.2105/AJPH.2011.300252

Kazianga, H., de Walque, D., \& Alderman, H. (2014). School feeding programs, intrahousehold allocation and the nutrition of siblings: Evidence from a randomized trial in rural Burkina Faso. Journal of Development Economics, 106, 15-34. doi:https://doi.org/10.1016/j.jdeveco.2013.08.007

Kien, V. D., Van Minh, H., Giang, K. B., Dao, A., Tuan, L. T., \& Ng, N. (2016). Socioeconomic inequalities in catastrophic health expenditure and impoverishment associated with non-communicable diseases in urban Hanoi, Vietnam. International Journal for Equity in Health, 15(1), 169. doi:10.1186/s12939-016-0460-3

Kino, S., Sato, K., \& Kawachi, I. (2018). Spillover benefit of improved access to healthcare on reducing worry about housing and meal affordability. International Journal for Equity in Health, 17(1), 174. doi:10.1186/s12939-018-0877-y

Knaul, F. M., Wong, R., Arreola-Ornelas, H., Méndez, O., Bitran, R., Campino, A. C., . . Rathe, M. (2011). Household catastrophic health expenditures: a comparative analysis of twelve Latin American and Caribbean countries. Salud pública de méxico, 53 (SUPPL.2), s85-s95. Retrieved from http://www.scopus.com/inward/record.url?scp=84857489365\&partnerID=8YFLogxK

Kuh, D. J. L., \& Wadsworth, M. E. J. (1993). Physical health status at 36 years in a British national birth cohort. Social Science \& Medicine, 37(7), 905-916. doi:https://doi.org/10.1016/0277-9536(93)90145-T

Ladinsky, J. L., \& Levine, R. E. (1985). The organization of health services in Vietnam. Journal of Public Health Policy, 6(2), 255-268. doi:10.2307/3342317

Le, N., Groot, W., Tomini, S. M., \& Tomini, F. (2019). Effects of health insurance on labour supply: evidence from the health care fund for the poor in Vietnam. Applied Economics, 51(58), 6190-6212. doi:10.1080/00036846.2019.1613509

Le, P., Griffiths, U. K., Anh, D. D., Franzini, L., Chan, W., Pham, H., \& Swint, J. M. (2014). The economic burden of pneumonia and meningitis among children less than five years old in Hanoi, Vietnam. Tropical Medicine \& International Health, 19(11), 1321-1327. doi:10.1111/tmi.12370

Le, T. D., \& Tran, N. M. T. (2013). Why children in Vietnam drop out of school and what they do after that. Young Lives Working Paper 102. Retrieved from https://www.younglives.org.uk/content/why-children-vietnam-drop-out-school-andwhat-they-do-after

Lee, H.-Y., Oh, J., Hoang, V. M., Moon, J. R., \& Subramanian, S. V. (2019). Use of high-level health facilities and catastrophic expenditure in Vietnam: can health insurance moderate this relationship? BMC Health Services Research, 19(1), 318. doi:10.1186/s12913-019-4115-0

Leininger, L., Levy, H., \& Schanzenbach, D. (2010). Consequences of SCHIP expansions for household well-being. Forum for Health Economics \& Policy, 13(1). doi:https://doi.org/10.2202/1558-9544.1201 
Levine, D., Polimeni, R., \& Ramage, I. (2016). Insuring health or insuring wealth? An experimental evaluation of health insurance in rural Cambodia. Journal of Development Economics, 119(Supplement C), 1-15. doi:https://doi.org/10.1016/j.jdeveco.2015.10.008

Levine, P. B., \& Schanzenbach, D. (2009). The impact of children's public health insurance expansions on educational outcomes. Forum for Health Economics \& Policy, 12(1). doi:10.2202/1558-9544.1137

Levy, H., \& Meltzer, D. (2008). The impact of health insurance on health. Annual Review of Public Health, 29(1), 399-409. doi:10.1146/annurev.publhealth.28.021406.144042

Lin, M.-J., \& Liu, E. M. (2014). Does in utero exposure to illness matter? The 1918 influenza epidemic in Taiwan as a natural experiment. Journal of Health Economics, 37, 152163. doi:https://doi.org/10.1016/j.jhealeco.2014.05.004

London, J. D. (2008). Reasserting the state in Viet Nam health care and the logics of marketLeninism. Policy and Society, 27(2), 115-128. doi:10.1016/j.polsoc.2008.09.005

Lu, F. (2014). Insurance coverage and agency problems in doctor prescriptions: Evidence from a field experiment in China. Journal of Development Economics, 106, 156-167. doi:https://doi.org/10.1016/j.jdeveco.2013.09.001

Lundberg, S. J., Pollak, R. A., \& Wales, T. J. (1997). Do husbands and wives pool their resources? Evidence from the United Kingdom child benefit. The Journal of Human Resources, 32(3), 463-480. doi:10.2307/146179

Manning, W. G., Newhouse, J. P., Duan, N., Keeler, E. B., \& Leibowitz, A. (1987). Health Insurance and the demand for medical care: Evidence from a randomized experiment. The American Economic Review, 77(3), 251-277. Retrieved from www.jstor.org/stable/1804094

Matsushima, M., \& Yamada, H. (2016). Impacts of bribery in healthcare in Vietnam. The Journal of Development Studies, 52(10), 1479-1498. doi:10.1080/00220388.2015.1121241

Matsushima, M., Yamada, H., \& Shimamura, Y. (2020). Analysis on demand- and supply-side responses during the expansion of health insurance coverage in Vietnam: Challenges and policy implications toward universal health coverage. Review of Development Economics, 24(1), 144-166. doi:10.1111/rode.12627

McGuire, T. G. (2000). Chapter 9 - Physician Agency. In A. J. Culyer \& J. P. Newhouse (Eds.), Handbook of Health Economics (Vol. 1, pp. 461-536): Elsevier.

McIntyre, D., Meheus, F., \& Røttingen, J.-A. (2017). What level of domestic government health expenditure should we aspire to for universal health coverage? Health Economics, Policy and Law, 12(2), 125-137. doi:10.1017/S1744133116000414

Meyer, B. D. (1995). Natural and quasi-experiments in economics. Journal of Business \& Economic Statistics, 13(2), 151-161. doi:10.2307/1392369

Miguel, E., \& Kremer, M. (2004). Worms: Identifying impacts on education and health in the presence of treatment externalities. Econometrica, 72(1), 159-217. doi:10.1111/j.1468-0262.2004.00481.x

Miller, G., Pinto, D., \& Vera-Hernandez, M. (2013). Risk protection, service use, and health outcomes under Colombia's Health Insurance Program for the Poor. American Economic Journal: Applied Economics, 5(4), 61-91. Retrieved from www.jstor.org/stable/43189453 
Miller, S., \& Wherry, L. R. (2019). The long-term effects of early life Medicaid coverage. Journal of Human Resources, University of Wisconsin Press, 54(3), 785-824. Retrieved from https://ideas.repec.org/a/uwp/jhriss/v54y2019i3p785-824.html

Ministry of Health of Vietnam, H. P. G. (2010). Joint Annual Health Review 2010: Vietnam's health system on the threshold of the five-year plan 2011-2015. Retrieved from Hanoi, Vietnam: http://jahr.org.vn/downloads/JAHR2010-EN.pdf

Ministry of Health of Vietnam, H. P. G. (2014). Join Annual Health Review 2014: strengthening prevention and control of non-communicable diseases. Retrieved from Hanoi, Vietnam: http://jahr.org.vn/downloads/JAHR2014/JAHR\%202014_EN_full.pdf

Mitra, S., Palmer, M., Mont, D., \& Groce, N. (2016). Can households cope with health shocks in Vietnam? Health Economics, 25(7), 888-907. doi:10.1002/hec.3196

Monheit, A. C., \& Vistnes, J. (2015). Does public health Insurance for children improve single mothers' health care use? Journal of Family and Economic Issues, 36(4), 581-592. doi:10.1007/s10834-014-9430-3

Nelson, R. E. (2010). Testing the fetal origins hypothesis in a developing country: evidence from the 1918 Influenza Pandemic. Health Economics, 19(10), 1181-1192. doi:10.1002/hec.1544

Nguyen, C. (2016). The impact of health insurance programs for children: evidence from Vietnam. Health Economics Review, 6(1), 34. doi:10.1186/s13561-016-0111-9

Nguyen, H., \& Wang, W. (2013). The effects of free government health insurance among small children-evidence from the free care for children under six policy in Vietnam. The International Journal of Health Planning and Management, 28(1), 3-15. doi:doi:10.1002/hpm.2114

Nguyen, H. G., Tran, T. M. O., Khuong, A. T., Phan, H. V., \& Jayasuriya, R. (2020). Is health insurance associated with health service utilization and economic burden of noncommunicable diseases on households in Vietnam? Health Systems \& Reform, 6(1), e1619065. doi:10.1080/23288604.2019.1619065

Nyman, J. A. (1999). The value of health insurance: the access motive. Journal of Health Economics, 18(2), 141-152. doi:https://doi.org/10.1016/S0167-6296(98)00049-6

Obermann, K., Jowett, M., \& Kwon, S. (2018). The role of national health insurance for achieving UHC in the Philippines: a mixed methods analysis. Global Health Action, 11(1), 1483638. doi:10.1080/16549716.2018.1483638

Ortiz-Ospina, E., \& Roser, M. (2019). Financing Healthcare. Published online at OurWorldInData.org. Retrieved from https://ourworldindata.org/financinghealthcare

Palmer, M., Mitra, S., Mont, D., \& Groce, N. (2014). The impact of health insurance for children under age 6 in Vietnam: A regression discontinuity approach. Social Science and Medicine. doi:10.1016/j.socscimed.2014.08.012

Palmer, M. G. (2014). Inequalities in Universal Health Coverage: Evidence from Vietnam. World Development, 64, 384-394. doi:https://doi.org/10.1016/j.worlddev.2014.06.008

Panpiemras, J., Puttitanun, T., Samphantharak, K., \& Thampanishvong, K. (2011). Impact of Universal Health Care coverage on patient demand for health care services in Thailand. Health Policy, 103(2), 228-235. doi:https://doi.org/10.1016/j.healthpol.2011.08.008

Parmar, D., Reinhold, S., Souares, A., Savadogo, G., \& Sauerborn, R. (2012). Does community-based health insurance protect household assets? Evidence from rural 
Africa. Health Services Research, 47(2), 819-839. doi:10.1111/j.1475-

6773.2011.01321.x

Pauly, M. V. (1968). The economics of moral hazard: Comment. The American Economic Review, 58(3), 531-537. Retrieved from www.jstor.org/stable/1813785

Peng, X., \& Conley, D. (2016). The implication of health insurance for child development and maternal nutrition: evidence from China. The European Journal of Health Economics, 17(5), 521-534. doi:10.1007/s10198-015-0696-7

Pfutze, T. (2014). The effects of Mexico's Seguro Popular Health Insurance on infant mortality: An estimation with selection on the outcome variable. World Development, 59, 475-486. doi:https://doi.org/10.1016/j.worlddev.2014.02.008

Pfutze, T. (2015). Does access to health insurance reduce the risk of miscarriages? Evidence from Mexico's Seguro popular. Latin American Economic Review, 24(1), 8. doi:10.1007/s40503-015-0022-x

Quimbo, S. A., Peabody, J. W., Shimkhada, R., Florentino, J., \& Solon, O. (2011). Evidence of a causal link between health outcomes, insurance coverage, and a policy to expand access: experimental data from children in the Philippines. Health Economics, 20(5), 620-630. doi:10.1002/hec.1621

Rangel, M. A. (2006). Alimony rights and intrahousehold allocation of resources: Evidence from Brazil. The Economic Journal, 116(513), 627-658. doi:10.1111/j.14680297.2006.01104.x \%J The Economic Journal

Rivera-Hernandez, M., Rahman, M., Mor, V., \& Galarraga, O. (2016). The impact of social health insurance on diabetes and hypertension process indicators among older adults in Mexico. Health Services Research, 51(4), 1323-1346. doi:10.1111/14756773.12404

Robinson, C. (2013). Younger siblings can be good for your health: An examination of spillover benefits from the Supplemental Nutrition Program for Women, Infants, and Children (WIC). Journal of Family and Economic Issues, 34(2), 172-184. doi:10.1007/s10834-012-9325-0

Robyn, P. J., Fink, G., Sié, A., \& Sauerborn, R. (2012). Health insurance and health-seeking behavior: Evidence from a randomized community-based insurance rollout in rural Burkina Faso. Social Science \& Medicine, 75(4), 595-603. doi:https://doi.org/10.1016/j.socscimed.2011.12.018

Robyn, P. J., Hill, A., Liu, Y., Souares, A., Savadogo, G., Sié, A., \& Sauerborn, R. (2011). Econometric analysis to evaluate the effect of community-based health insurance on reducing informal self-care in Burkina Faso. Health Policy and Planning, 27(2), 156165. doi:10.1093/heapol/czr019

Rothschild, M., \& Stiglitz, J. (1978). Equilibrium in competitive insurance markets: An essay on the economics of imperfect information. In Uncertainty in economics (pp. 257280): Elsevier.

Rubalcava, L., Teruel, G., \& Thomas, D. (2009). Investments, time preferences, and public transfers paid to women. Economic Development and Cultural Change, 57(3), 507538. doi:10.1086/596617

Schmidt, W.-P., Suzuki, M., Vu, D. T., Yoshida, L.-M., Matsubayashi, T., Yanai, H., . . Ariyoshi, K. (2012). User fee exemption does not affect lower rates of hospital admission of girls in Vietnam. Health Policy and Planning, 27(7), 582-589.

doi:10.1093/heapol/czr079 
Sepehri, A., Sarma, S., \& Oguzoglu, U. (2011). Does the financial protection of health insurance vary across providers? Vietnam's experience. Social Science \& Medicine, 73(4), 559-567. doi:https://doi.org/10.1016/j.socscimed.2011.06.009

Sepehri, A., Sarma, S., \& Serieux, J. (2009). Who is giving up the free lunch? The insured patients' decision to access health insurance benefits and its determinants: Evidence from a low-income country. Health Policy, 92(2), 250-258. doi:https://doi.org/10.1016/j.healthpol.2009.05.005

Sheu, J.-T., \& Lu, J.-f. R. (2014). The spillover effect of National Health Insurance on household consumption patterns: Evidence from a natural experiment in Taiwan. Social Science \& Medicine, 111, 41-49. doi:https://doi.org/10.1016/j.socscimed.2014.04.006

Shieh, M., Thompson, C., Tra, M. P. V., Linh, V. T. T., Tediosi, F., Merson, L., . . Baker, S. (2013). The policy of free healthcare for children under the age of 6 years in Vietnam: assessment of the uptake for children hospitalised with acute diarrhoea in Ho Chi Minh City. Tropical Medicine \& International Health, 18(12), 1444-1451. doi:10.1111/tmi.12208

Sood, N., Bendavid, E., Mukherji, A., Wagner, Z., Nagpal, S., \& Mullen, P. (2014). Government health insurance for people below poverty line in India: quasiexperimental evaluation of insurance and health outcomes. BMJ : British Medical Journal, 349, g5114. doi:10.1136/bmj.g5114

Sparrow, R., Suryahadi, A., \& Widyanti, W. (2013). Social health insurance for the poor: Targeting and impact of Indonesia's Askeskin programme. Social Science \& Medicine, 96, 264-271. doi:https://doi.org/10.1016/j.socscimed.2012.09.043

Strauss, J., \& Thomas, D. (2007). Chapter 54 Health over the Life Course. In T. P. Schultz \& J. A. Strauss (Eds.), Handbook of Development Economics (Vol. 4, pp. 3375-3474): Elsevier.

Takashima, K., Wada, K., Tra, T. T., \& Smith, D. R. (2017). A review of Vietnam's healthcare reform through the Direction of Healthcare Activities (DOHA). Environmental Health and Preventive Medicine, 22(1), 74. doi:10.1186/s12199-017-0682-z

Thomas, D. (1990). Intra-household resource allocation: An inferential approach. The Journal of Human Resources, 25(4), 635-664. doi:10.2307/145670

Thomas, D., \& Strauss, J. (1997). Health and wages: Evidence on men and women in urban Brazil. Journal of Econometrics, 77(1), 159-185. doi:https://doi.org/10.1016/S03044076(96)01811-8

Treleaven, E., Toan, P. N., Le, D. N., Diamond-Smith, N., Partridge, J. C., \& Le, H. T. (2016). Gender disparities in child health care seeking in northern Vietnam. Asian Population Studies, 12(3), 312-330. doi:10.1080/17441730.2016.1207930

Trujillo, A. J., Ortiz, A. I. V., Gómez, F. R., \& Steinhardt, L. C. (2010). Health insurance doesn't seem to discourage prevention among diabetes patients in Colombia. Health Affairs, 29(12), 2180-2188. doi:https://doi.org/10.1377/hlthaff.2010.0463

Turley, W. S., \& Selden, M. (2019). Reinventing Vietnamese socialism: Doi Moi in comparative perspective. New York: Routledge.

Van Minh, H., Kim Phuong, N. T., Saksena, P., James, C. D., \& Xu, K. (2013). Financial burden of household out-of pocket health expenditure in Viet Nam: Findings from the National Living Standard Survey 2002-2010. Social Science \& Medicine, 96, 258-263. doi:https://doi.org/10.1016/j.socscimed.2012.11.028 
Van Tien, T., Phuong, H. T., Mathauer, I., \& Phuong, N. T. K. (2011). A health financing review of Vietnam World Health Organization. Retrieved from https://www.who.int/health_financing/documents/cov-oasis_e_11-vietnam/en/

Vartanian, T. P., \& Houser, L. (2012). The effects of childhood SNAP use and neighborhood conditions on adult Body Mass Index. Demography, 49(3), 1127-1154. doi:10.1007/s13524-012-0115-y

Ver Ploeg, M. (2009). Do benefits of U.S. food assistance programs for children spillover to older children in the same household? Journal of Family and Economic Issues, 30(4), 412. doi:10.1007/s10834-009-9164-9

Vu, H. L., \& Sharrock, P. (2015). Descending dragon, rising Tiger : A history of Vietnam. London, UNITED KINGDOM: Reaktion Books, Limited.

$\mathrm{Vu}, \mathrm{K}$. (2019). Does universal health coverage at early age reduce medical needs at later age? Evidence from Vietnam. doi:http://dx.doi.org/10.2139/ssrn.3409107

Vujicic, M., Shengelia, B., Alfano, M., \& Thu, H. B. (2011). Physician shortages in rural Vietnam: Using a labor market approach to inform policy. Social Science \& Medicine, 73(7), 970-977. doi:https://doi.org/10.1016/j.socscimed.2011.06.010

Wagstaff, \& Adam Bales, S. (2012). The impacts of public hospital autonomization: Evidence from a quasi-natural experiment. Policy Research Working Papers: The World Bank. Washington DC: The World Bank.

Wagstaff, A. (2007a). Health insurance for the poor : Initial impacts of Vietnam's Health Care Fund for the Poor. Policy Research Working Papers: The World Bank. Washington DC: The World Bank.

Wagstaff, A. (2007b). The economic consequences of health shocks: Evidence from Vietnam. Journal of Health Economics, 26(1), 82-100. doi:https://doi.org/10.1016/j.jhealeco.2006.07.001

Wagstaff, A. (2010). Estimating health insurance impacts under unobserved heterogeneity: the case of Vietnam's health care fund for the poor. Health Economics, 19(2), 189208. doi:10.1002/hec.1466

Wagstaff, A., Cotlear, D., Eozenou, P. H.-V., \& Buisman, L. R. (2016). Measuring progress towards universal health coverage: with an application to 24 developing countries. Oxford Review of Economic Policy, 32(1), 147-189. doi:10.1093/oxrep/grv019

Wagstaff, A., \& Doorslaer, E. v. (2003). Catastrophe and impoverishment in paying for health care: with applications to Vietnam 1993-1998. Health Economics, 12(11), 921-933. doi:10.1002/hec.776

Wagstaff, A., Flores, G., Hsu, J., Smitz, M.-F., Chepynoga, K., Buisman, L. R., . . Eozenou, P. (2018). Progress on catastrophic health spending in 133 countries: a retrospective observational study. The Lancet Global Health, 6(2), e169-e179. doi:https://doi.org/10.1016/S2214-109X(17)30429-1

Wagstaff, A., Nguyen, H. T. H., Dao, H., \& Bales, S. (2016). Encouraging health insurance for the informal sector: A cluster randomized experiment in Vietnam. Health Economics, 25(6), 663-674. doi:10.1002/hec.3293

Wagstaff, A., \& Pradhan, M. (2005). Health insurance impacts on health and nonmedical consumption in a developing country. World Bank Policy Research Working Paper No. 3563 (Vol. 3563). Washington DC: World Bank Publications.

Wagstaff, A., \& van Doorslaer, E. (2001). Paying for health care: Quantifying fairness, catastrophe, and impoverishment, with applications to Vietnam, 1993-98. Policy Research Working Papers. Washington DC: The World Bank. 
Wherry, L. R., \& Meyer, B. D. (2016). Saving teens: using a policy discontinuity to estimate the effects of Medicaid eligibility. Journal of Human Resources, University of Wisconsin Press, 51(3), 556-588. doi:10.3368/jhr.51.3.0913-5918R1

WHO. (2005). Sustainable health financing universal coverage and social health insurance. World Health Assembly Resolution 58.33. Retrieved from https://www.who.int/health_financing/documents/cov-wharesolution5833/en/

Wooldridge, J. M. (2012). Introductory econometrics : a modern approach: Fifth edition. Mason, Ohio : South-Western Cengage Learning.

WorldBank. (2011). Phân tích việc thực hiện Chính sách tự chủ bệnh viện trên thế giới và thực tế ở Việt Nam. Retrieved from http://documents.worldbank.org/curated/en/289261468125691088/pdf/660140VIE TNAMEOLessonsOforOHospital.pdf

WorldBank. (2019). World Bank - World Development Indicators. Retrieved from: http://data.worldbank.org/data-catalog/world-development-indicators

Zijdeman, R., \& Ribeira da Silva, F. (2015). Life Expectancy at Birth (Total). Retrieved from: https://hdl.handle.net/10622/LKYT53

Ziol - Guest, K. M., \& Dunifon, R. E. J. F. R. (2014). Complex living arrangements and child health: Examining family structure linkages with children's health outcomes. Family Relations, 63(3), 424-437. 


\section{Appendix}

Figure 2. 1 Economic Growth in Vietnam from 1984 to 2017

\section{GDP per capita in US\$}

Gross domestic product per capita adjusted for price changes over time (inflation) and expressed in US-Dollars.

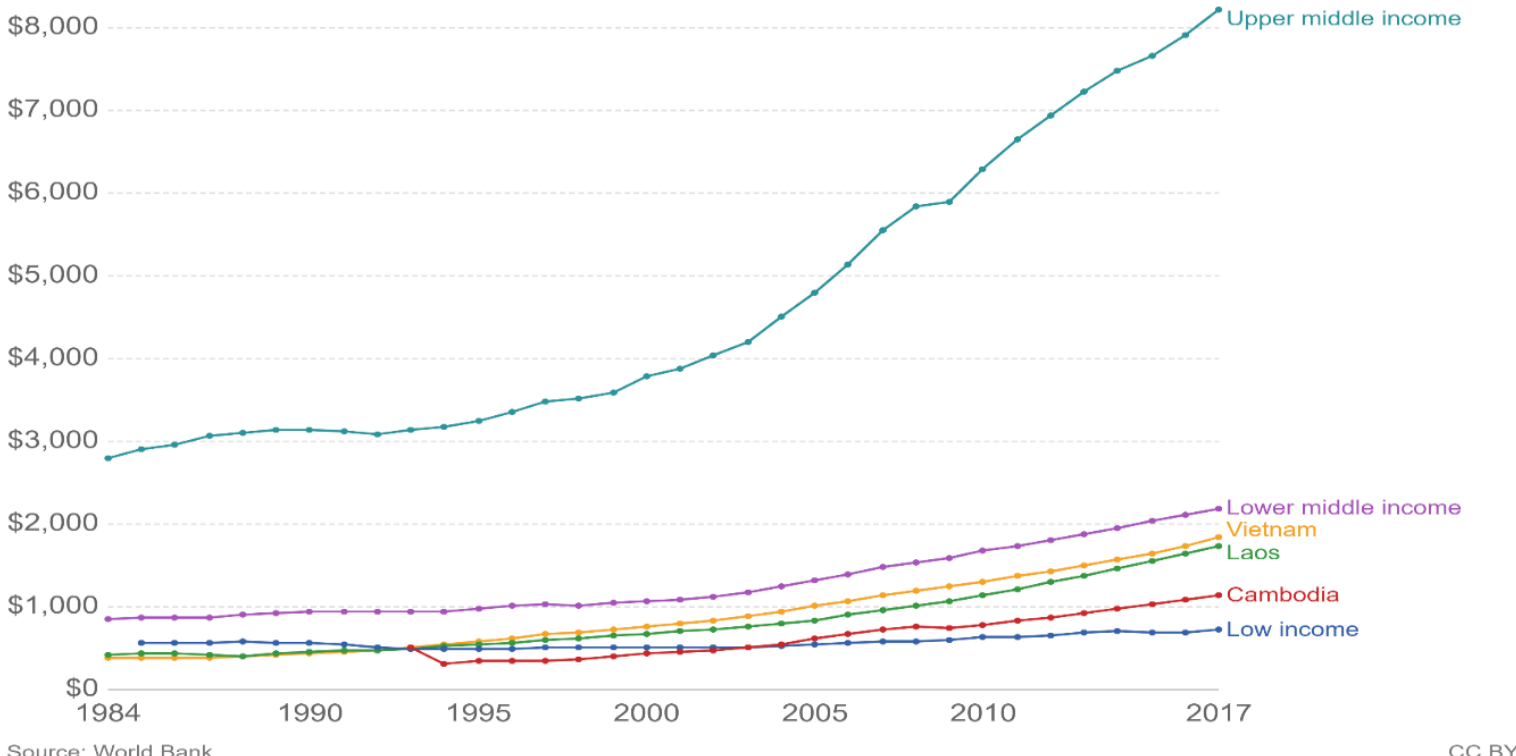

Figure 2. 2 Urbanization in Vietnam

Share of people living in urban areas

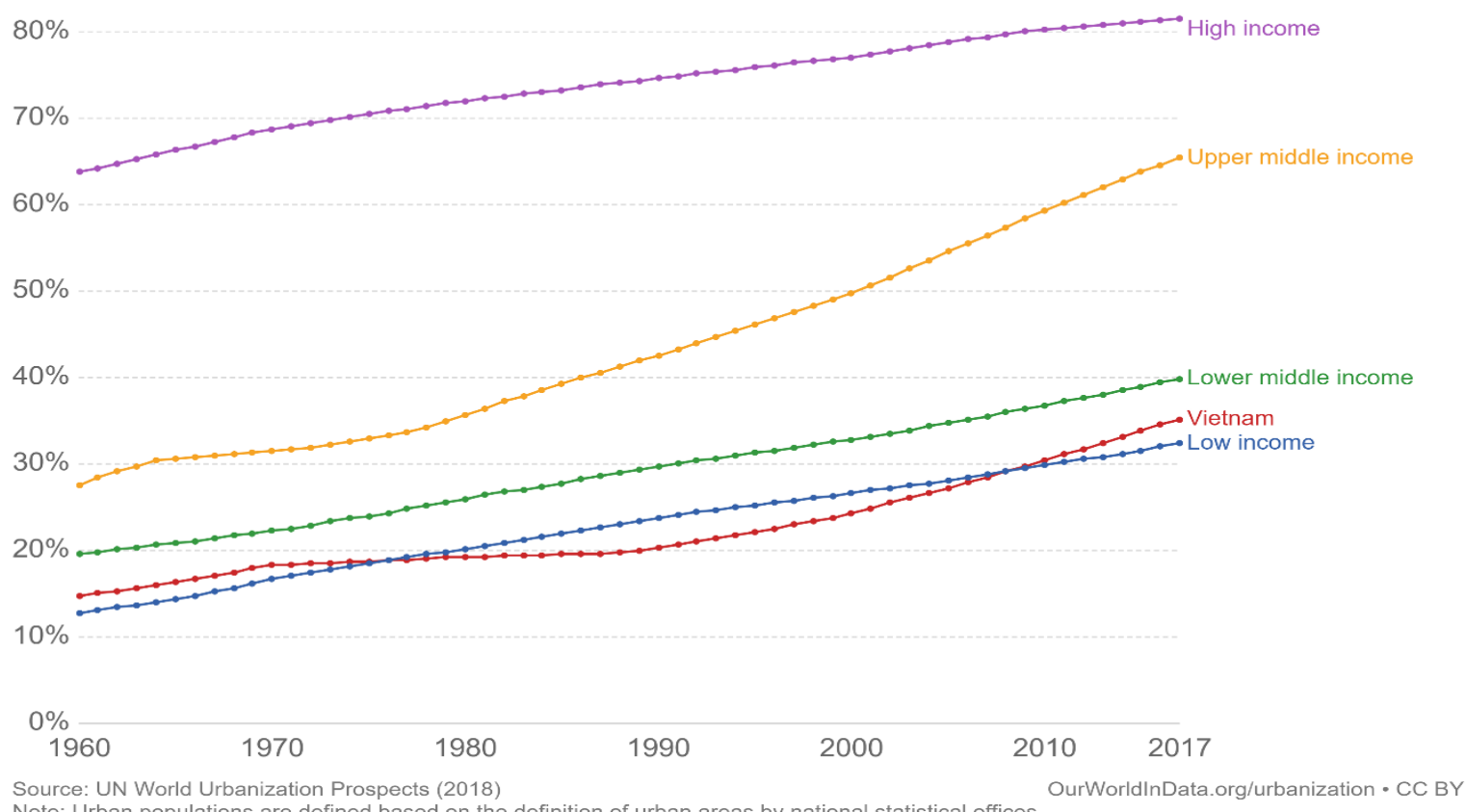

Note: Urban populations are defined based on the definition of urban areas by national statistical offices. 
Figure 2. 3 Life expectancy in Vietnam

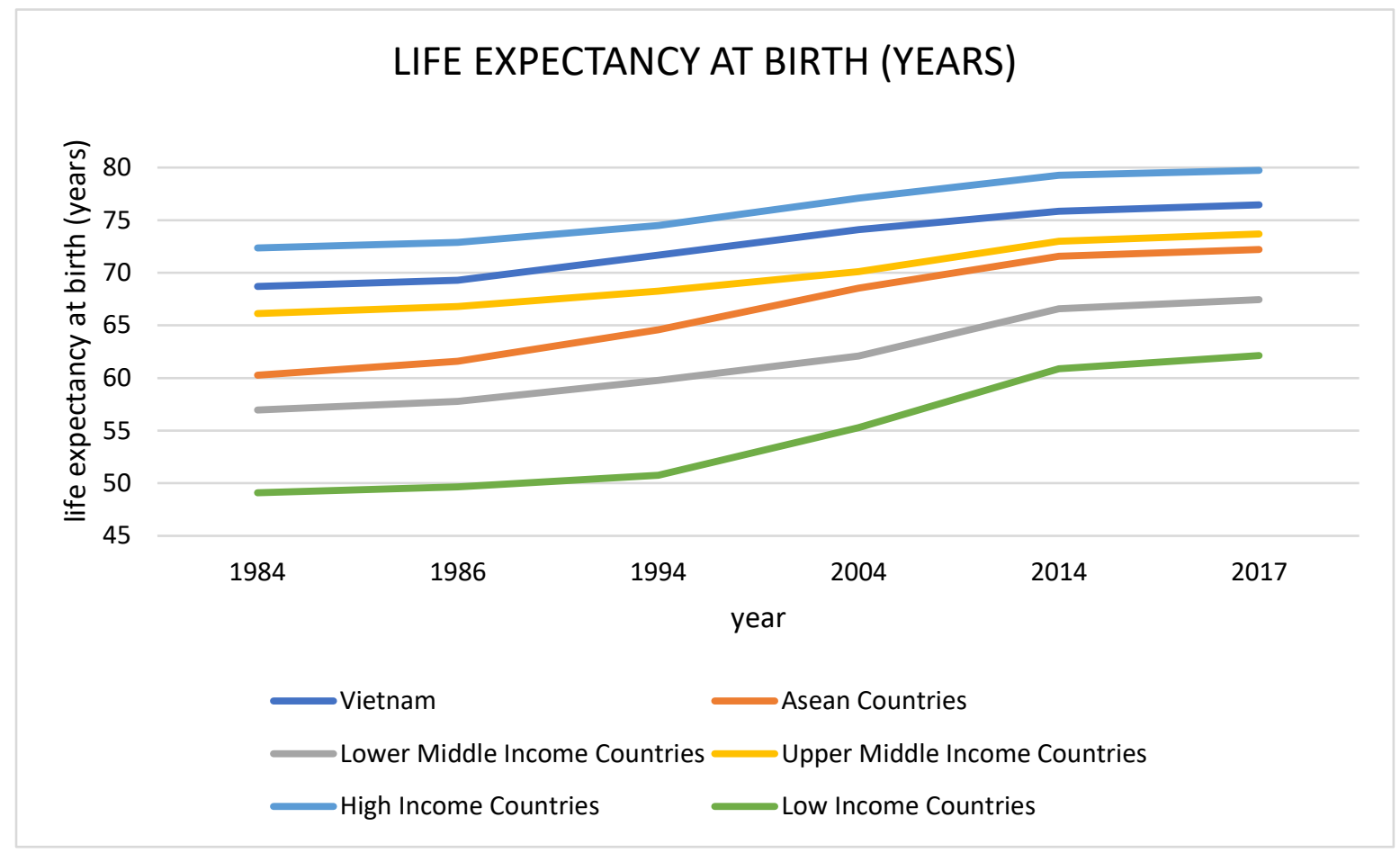

Figure 2. 4 Infant Mortality Rate in Vietnam

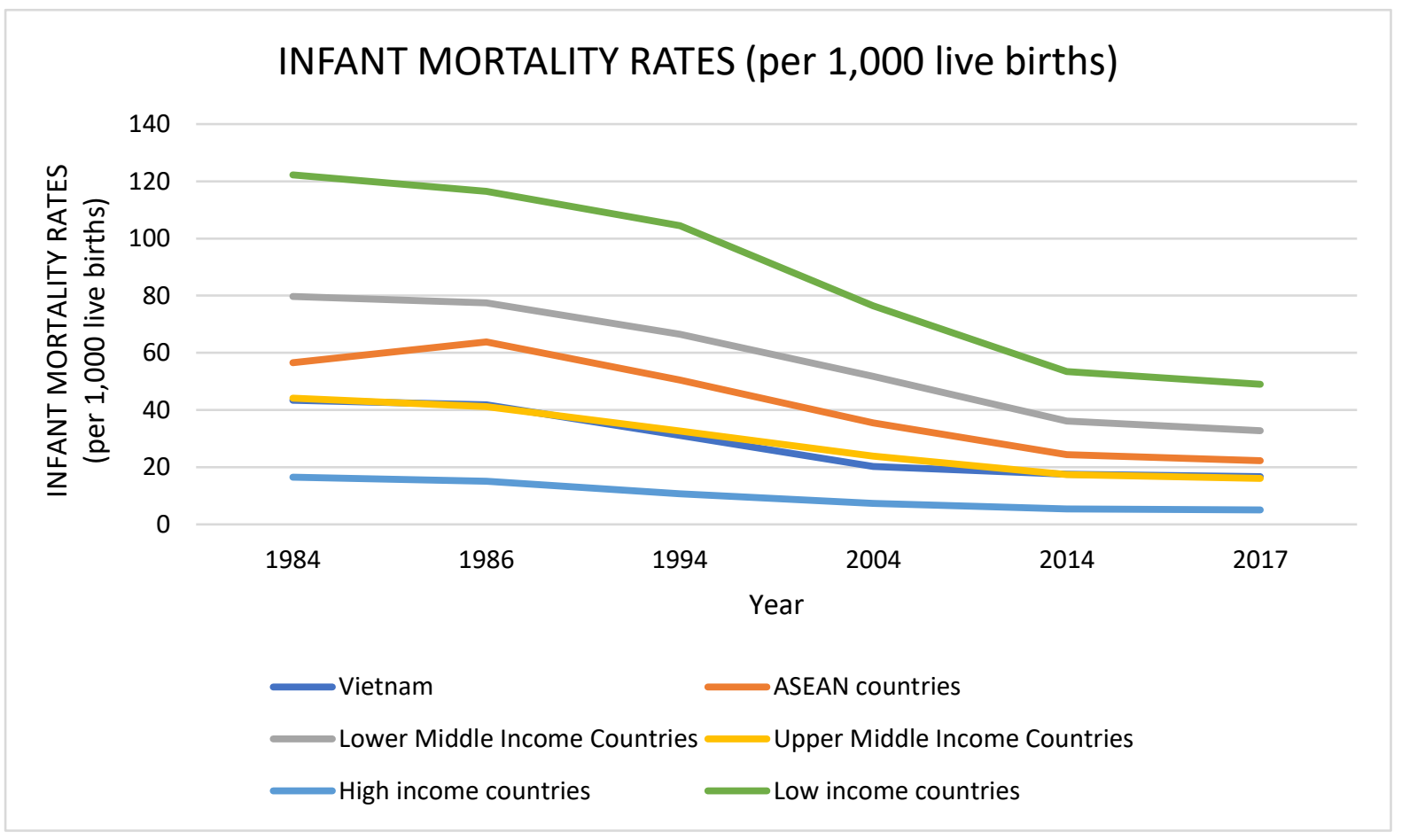


Figure 2. 5 Malnutrition prevalence in Vietnam - Height for Age

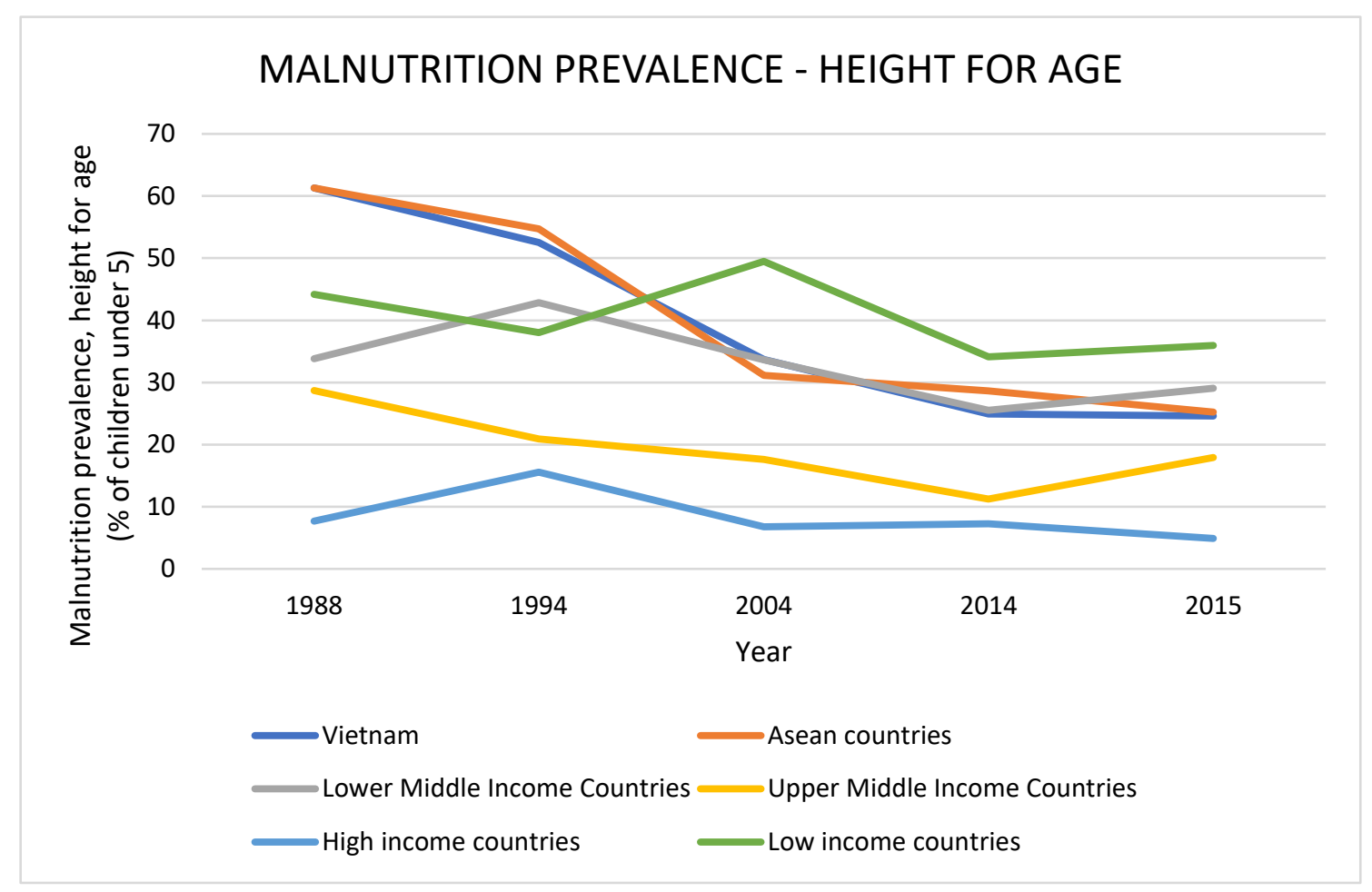

Figure 2. 6 Malnutrition prevalence in Vietnam - Weight for Age

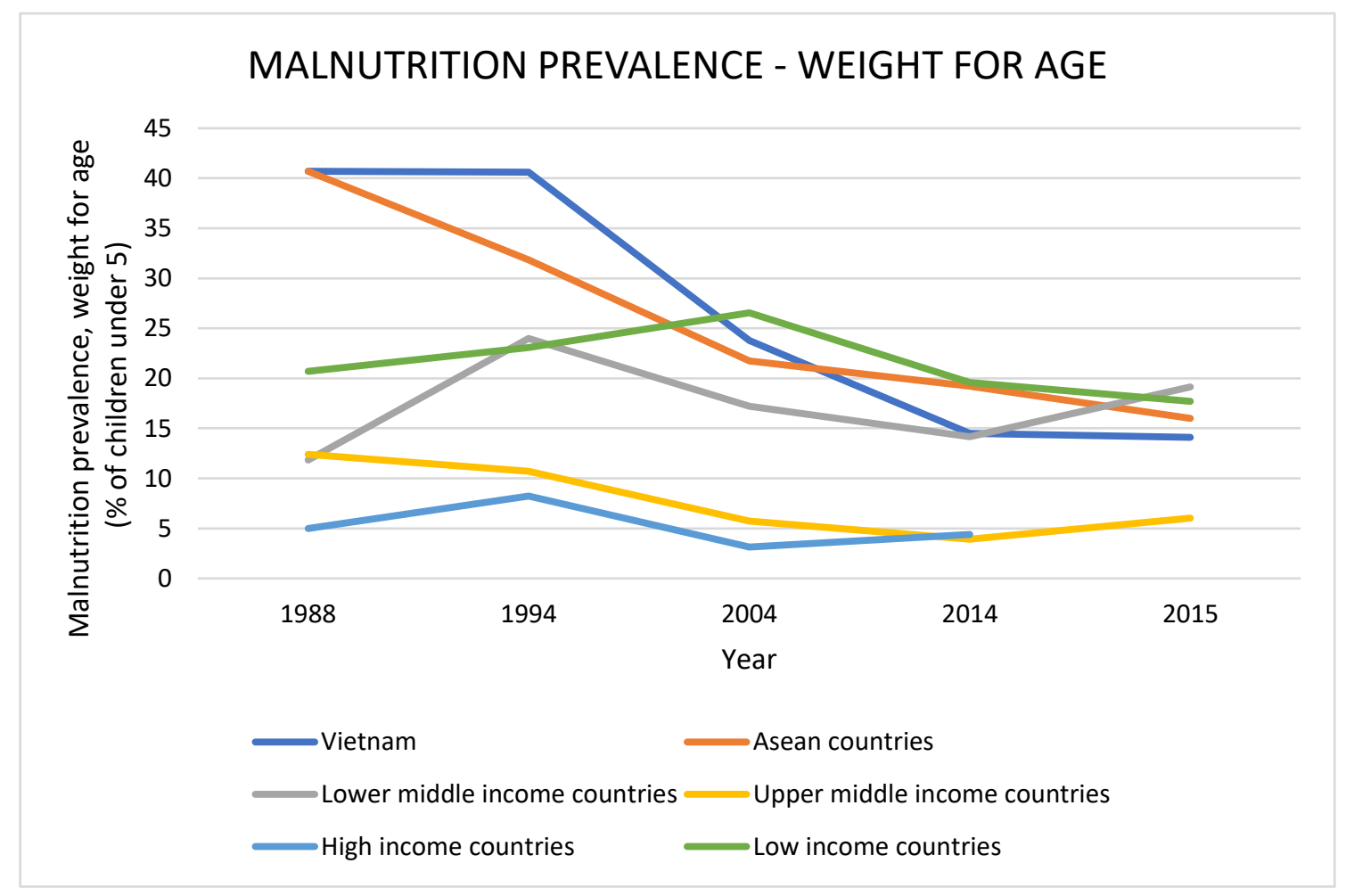


Figure 2. 7 Healthcare expenditure in Vietnam

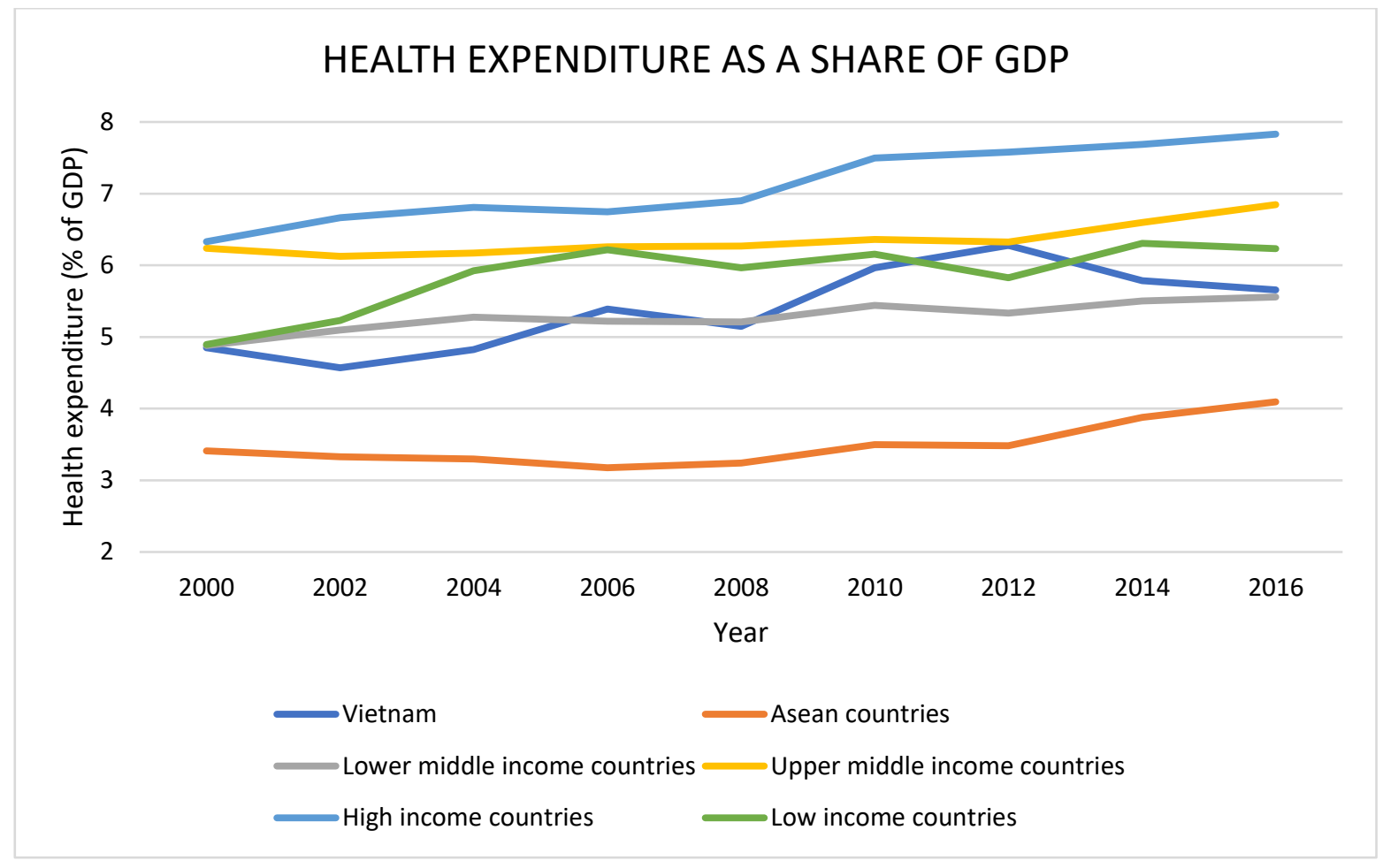


Figure 2. 8 Primary types of health care facilities in Vietnam

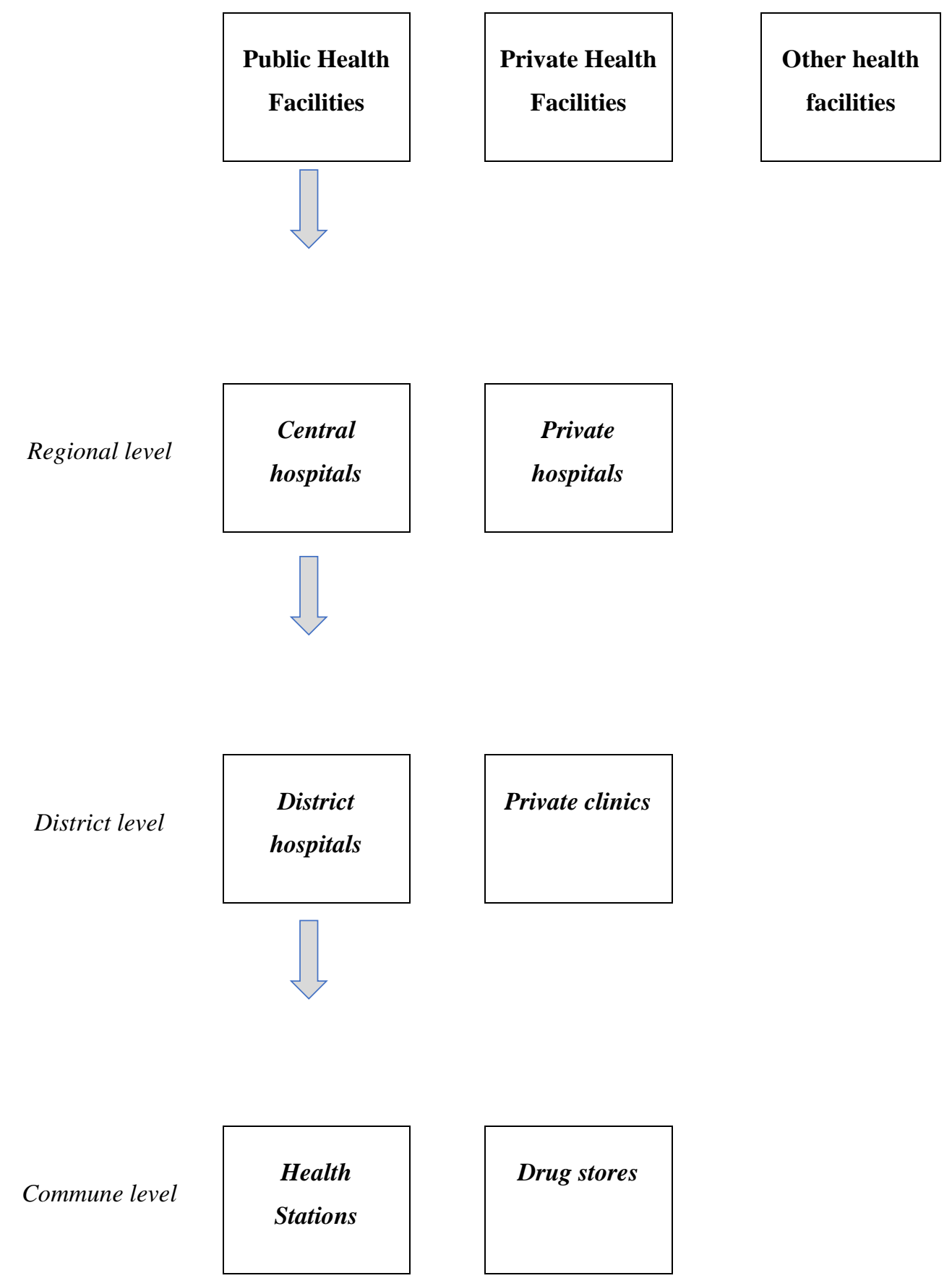


Figure 2. 9 The number of hospital beds during the last three decades (data from the Vietnam Health Statistics Yearbook, 2016)

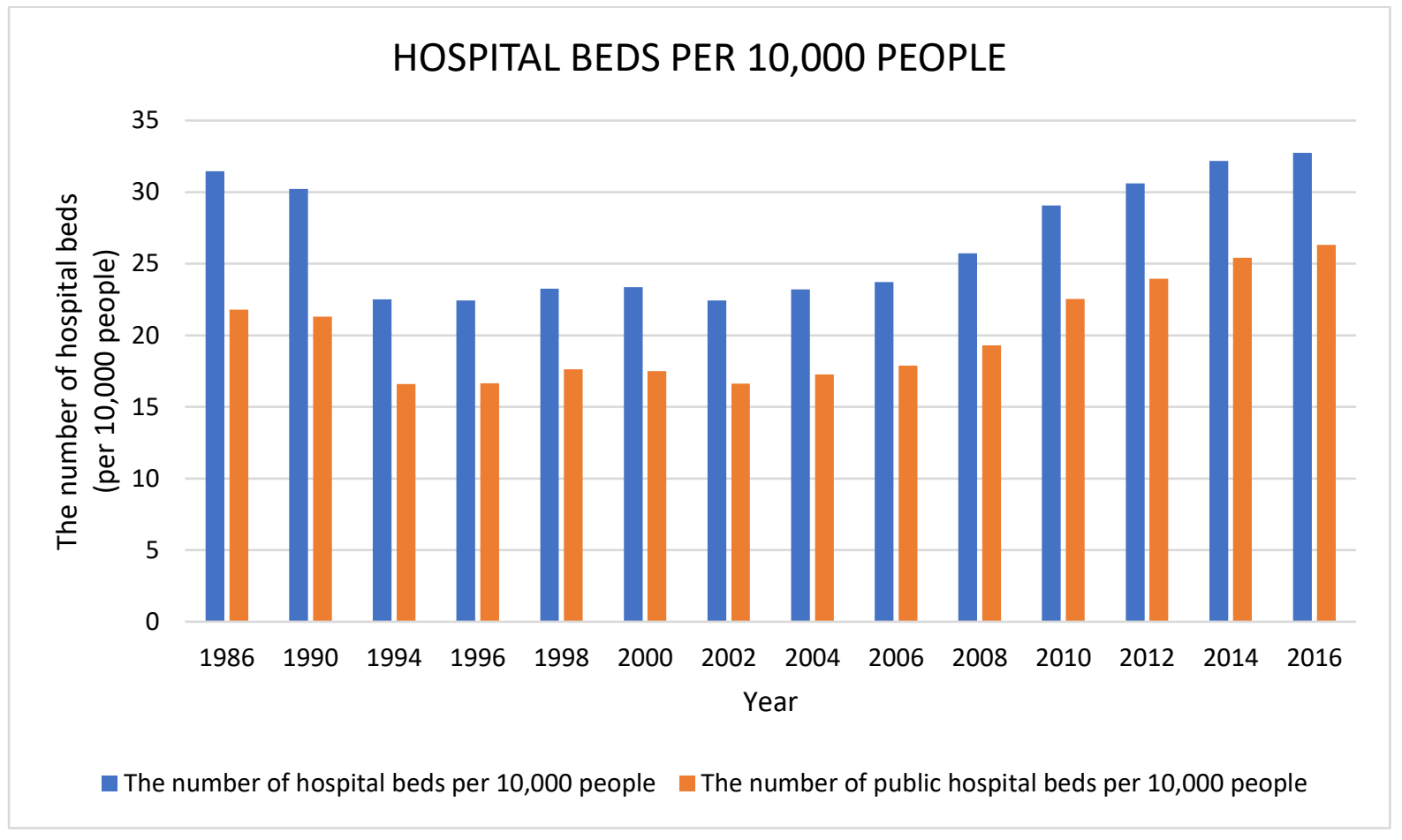

Figure 2. 10 Morbidity trend by types of disease (data from the Vietnam Health Statistics Yearbook, 2016)

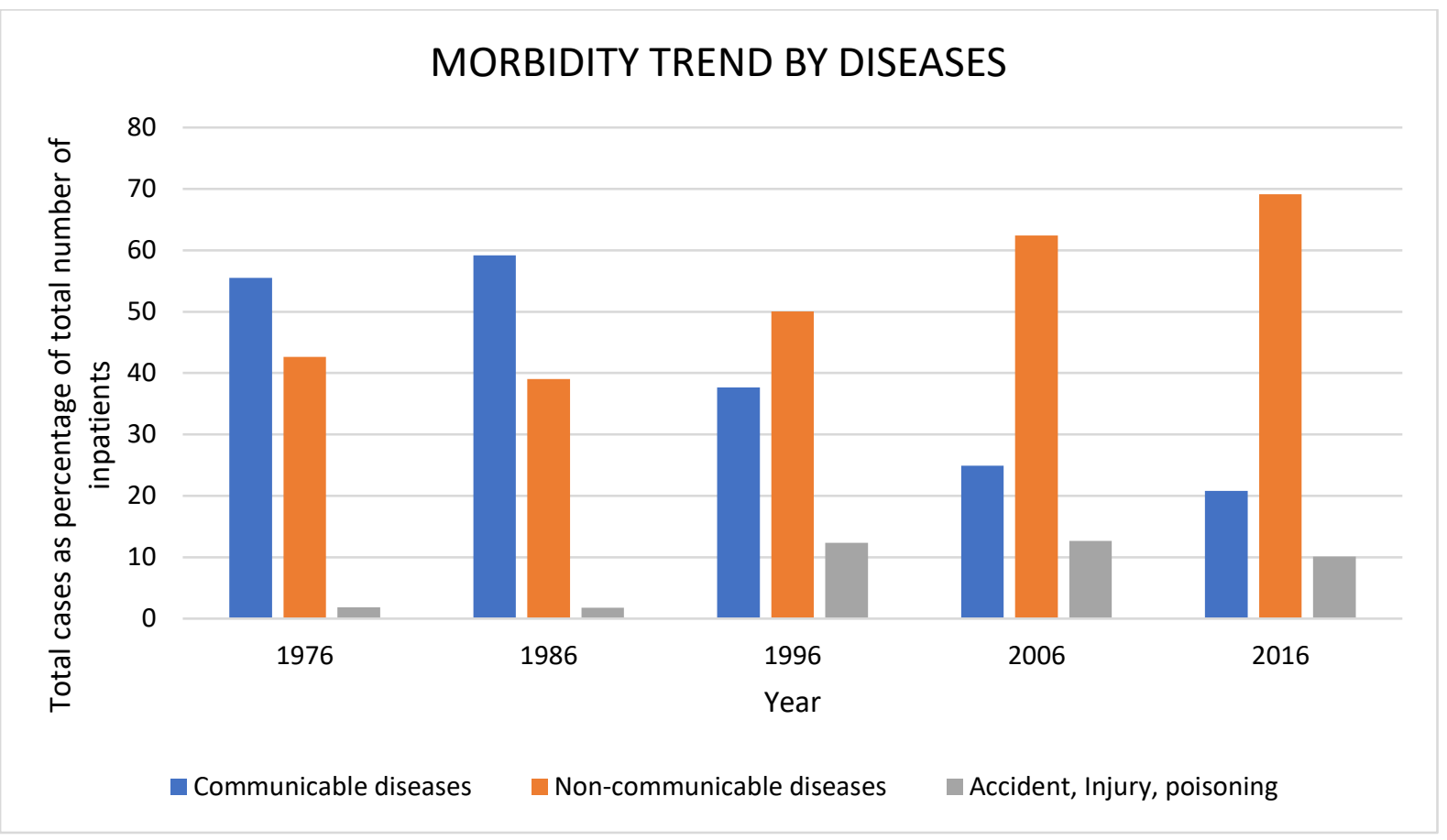


Figure 2. 11 Mortality trend by types of diseases (data from the Vietnam Health Statistics Yearbook, 2016)

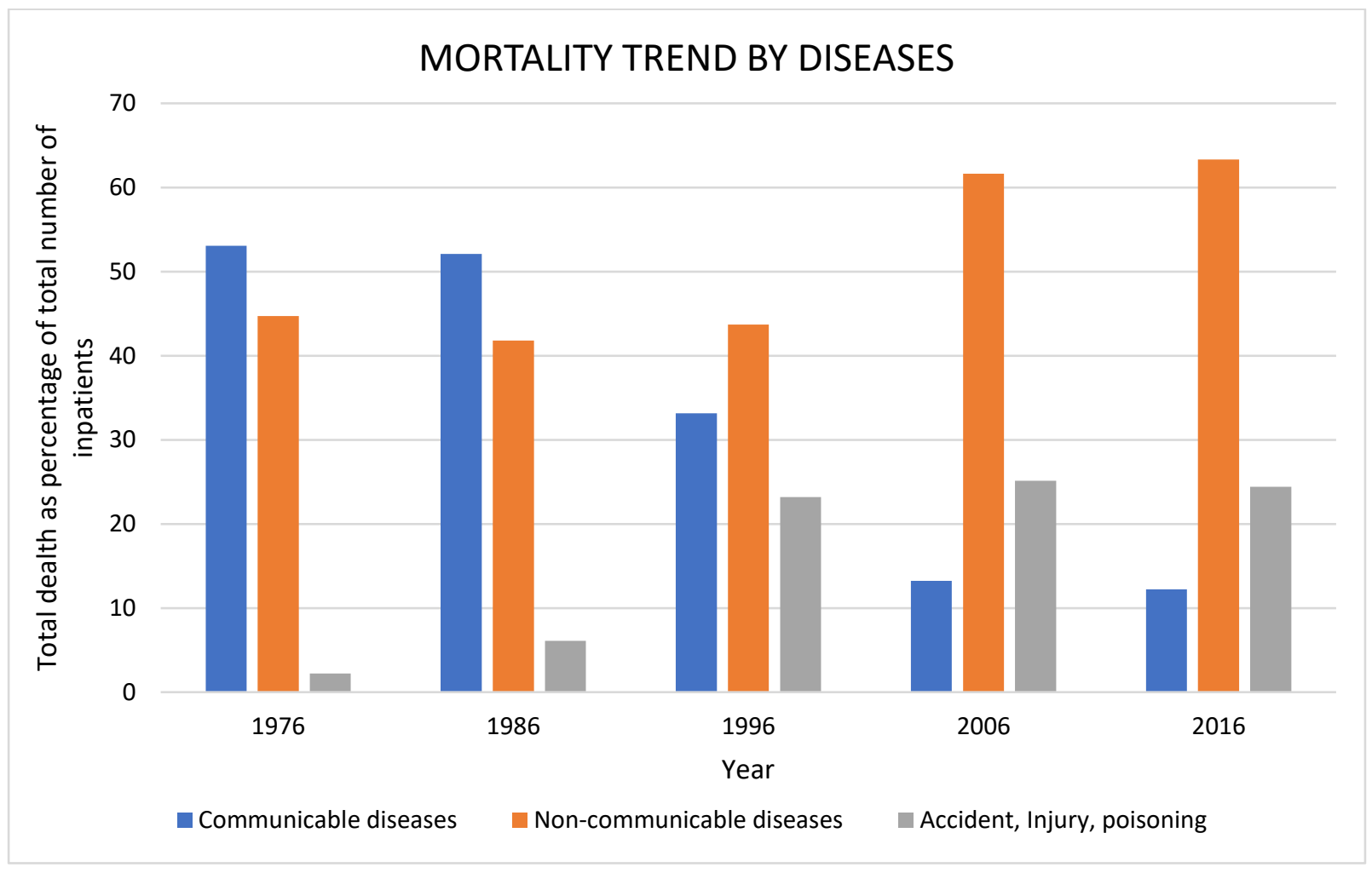


Figure 2. 12 A brief history of health insurance in Vietnam

\begin{tabular}{|c|c|c|c|c|}
\hline \multicolumn{2}{|c|}{ Social Health Insurance } & \multirow[t]{2}{*}{ Year } & \multicolumn{2}{|l|}{ Private Health Insurance } \\
\hline Coverage & Policy change & & Policy change & Coverage \\
\hline & & 1964 & Policy 179/CP: First insurance company Bao Viet established & \\
\hline & The country gain independence, centrally planned economy & 1975 & $\begin{array}{l}\text { The country gain independence, centrally planned economy, abolished private insurance } \\
\text { companies in the South }\end{array}$ & \\
\hline & & 1976 & Policy 21/QĐ-BKT: Established BAVINA insurance in the South of Vietnam & \\
\hline & & 1977 & $\begin{array}{l}\text { Policy 61/TCQĐ/TCCB: Merged BAVINA with Bao Viet company, official brought Bao } \\
\text { Viet to the South }\end{array}$ & \\
\hline & Major political reform, market-oriented economy & 1986 & Major political reform, market oriented economy & \\
\hline & Law on health protection for citizen & 1989 & Policy 27/TCQĐ/TCCB: Established Vietnam Insurance Company & \\
\hline & Policy 45/HĐBT: Introduced partial user fees at hospitals & & & \\
\hline & Policy 299/HĐBT: Introduced social compulsory and voluntary health insurance & 1992 & & \\
\hline & Policy 958/BYT-QĐ: Established Vietnam health insurance & & & \\
\hline \multirow[t]{4}{*}{$5.40 \%$} & & 1993 & Policy 100/CP: Regulation on insurance business, many insurance companies established & \\
\hline & Policy 14/TTLB: Introduced voluntary health insurance for students & 1994 & & \\
\hline & Policy 95/CP: Regulated hospital fees, fee exemption applied & 1995 & & \\
\hline & & 1996 & Policy 02-TC/TCNH: First introduced life insurance offered by Bao Viet company & \\
\hline \multirow[t]{6}{*}{$12.50 \%$} & $\begin{array}{l}\text { Policy 58/1998/NĐ-CP: Regulated health insurance, introduce 20\% co-payment } \\
\text { rate }\end{array}$ & 1998 & & \\
\hline & & 1999 & $\begin{array}{l}\text { Certificate 2122/GP: Allowing the first foreign insurance company to run business in } \\
\text { Vietnam }\end{array}$ & \\
\hline & & 2000 & Law on Insurance Business & \\
\hline & Policy 30/2002/NĐ-CP: Regulation on health care for the elder & 2002 & & \\
\hline & $\begin{array}{l}\text { Policy 100/2002/NĐ-CP: Merging health insurance into social security } \\
\text { insurance }\end{array}$ & & & \\
\hline & Policy 10/2002 - NĐ-CP: Hospital autonomy & & & \\
\hline \multirow[t]{2}{*}{$\begin{array}{l}20 \% \\
(2003)\end{array}$} & $\begin{array}{l}\text { Policy 139/2002/QĐ-TTg: Social compulsory health insurance for the poor } \\
\text { using Health care Fund for the poor }{ }^{(1)}\end{array}$ & & & \\
\hline & Policy 46/NQ-TW: Achieved universal social health insurance in 2010 & 2005 & & \\
\hline \multirow[t]{2}{*}{$28.40 \%$} & Policy 36/2005/NĐ-CP: Free health care for children under 6 at public hospitals & & & $4 \%$ \\
\hline & Policy 03/2006/TTLT-BYT-BTC-BLĐTB\&XH: Updated hospital fees & & & \\
\hline $41 \%$ & Policy 43/2006/NĐ-CP: Hospital Autonomy & 2006 & & $4 \%$ \\
\hline $46 \%$ & $\begin{array}{l}\text { Policy 10/2008/TTLT-BYT-BTC: Government subsidized at least 50\% of } \\
\text { premiums for the near-poor }{ }^{(2)}\end{array}$ & 2008 & & $5 \%$ \\
\hline
\end{tabular}


Law on Health Insurance

Law on Health Insurance: Social compulsory health insurance for students

2010

\begin{tabular}{|c|c|c|c|}
\hline $\mathbf{5 8 . 2 0 \%}$ & Law on Health Insurance: Social compulsory health insurance for students & 2010 & $5 \%$ \\
\hline \multirow[t]{3}{*}{$66.8 \%{ }^{(4)}$} & Policy 04/2012/TTLT-BYT-BTC: Updated hospital fees & 2012 & $5 \%$ \\
\hline & $\begin{array}{l}\text { Policy 10/2008/TTLT-BYT-BTC: Government subsidized at least 70\% of } \\
\text { premiums for the near-poor }^{(3)}\end{array}$ & & \\
\hline & Law on Health Insurance: Social compulsory health insurance for farmers & & \\
\hline $70 \%$ & $\begin{array}{l}\text { Policy 538/QĐ-TTg: Roadmap to achieve UHIC in 2020, reduce out-of-pocket } \\
\text { payment to below } 40 \% \text { by } 2020\end{array}$ & 2013 & $6 \%$ \\
\hline \multicolumn{4}{|l|}{$\begin{array}{l}\mathbf{8 0 \%} \\
(\mathbf{2 0 2 0})\end{array}$} \\
\hline & $\begin{array}{l}\text { Law on Health Insurance: Social compulsory for household member of } \\
\text { employees }\end{array}$ & 2014 & $6 \%$ \\
\hline
\end{tabular}

private insurance companies 
Figure 2. 13 Out-of-pocket payments as a share of national health expenditures (data from the World Bank)

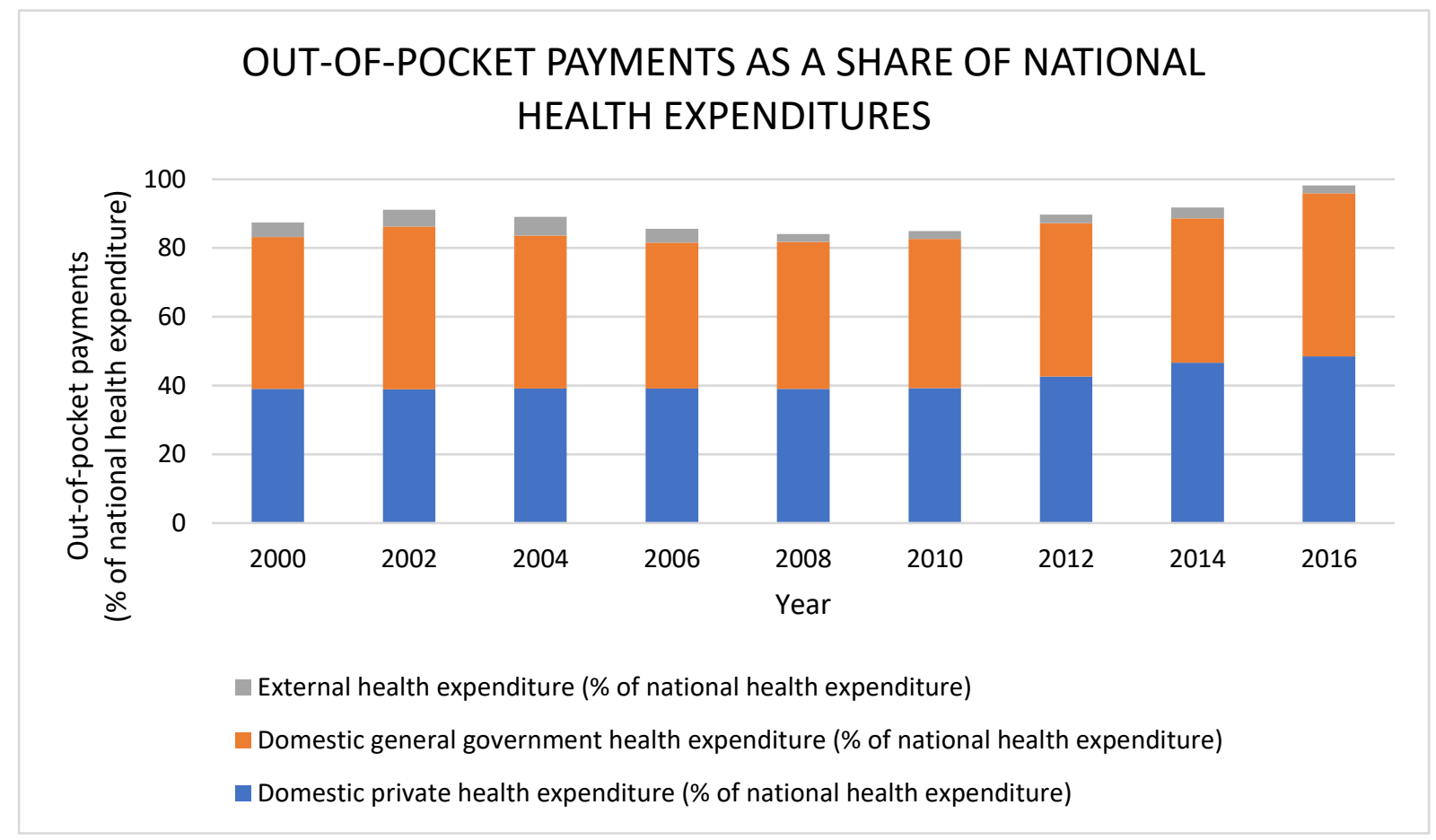

Figure 2. 14 Impoverishment due to illness (data from the World Bank)

\section{IMPOVERISHMENT DUE TO ILLNESS}

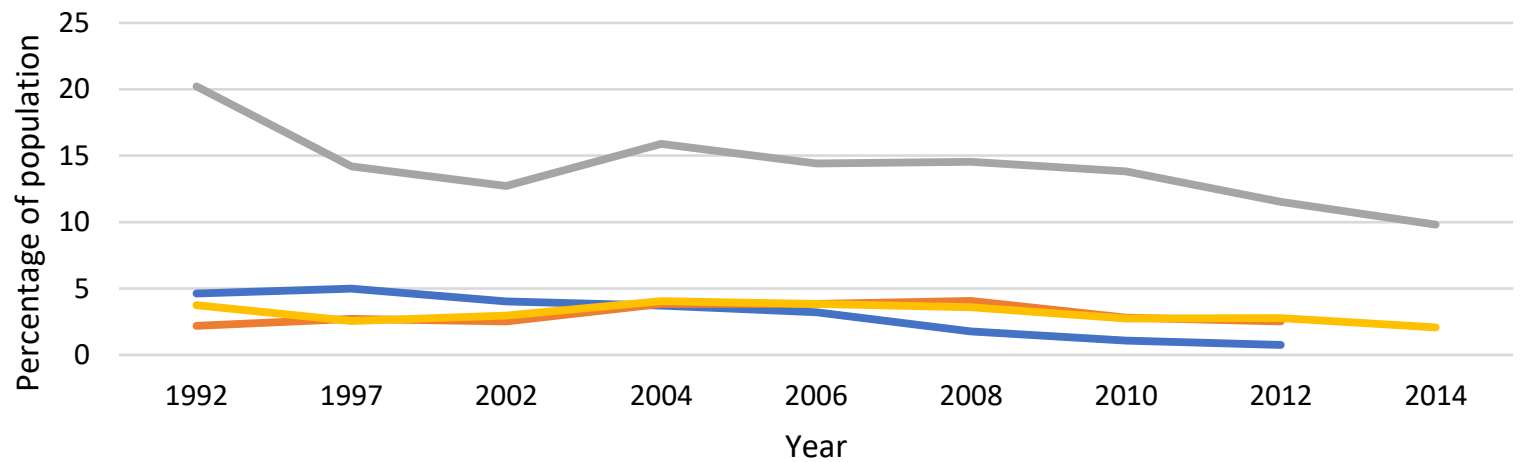

Proportion of population pushed below the \$1.90 (\$2011 PPP) poverty line by out-of-pocket health care expenditure (\%)

Proportion of population pushed below the $\$ 3.10$ (\$2011 PPP) poverty line by out-of-pocket health care expenditure (\%)

Proportion of population spending more than $10 \%$ of household consumption or income on out-ofpocket health care expenditure (\%)

Proportion of population spending more than $25 \%$ of household consumption or income on out-ofpocket health care expenditure (\%) 